\title{
DEVELOPMENT OF AN ELECTROMAGNETIC ACTUATOR BASED TRANSLATION MICROMIRROR \\ AS A MOVABLE MIRROR IN A MINIATURIZED FTIR SPECTROMETER
}

\author{
By \\ Yuan Xue \\ A dissertation \\ presented to Ryerson University \\ in partial fulfillment of the \\ requirements for the degree of \\ Doctor of Philosophy \\ in the program of
}

BEng, Mechanical Engineering, Ryerson University, 2009

MASc, Mechanical Engineering, Ryerson University, 2011

Mechanical and Industrial Engineering

Toronto, Ontario, Canada, 2018

(C) Yuan Xue, 2018 


\begin{abstract}
AUTHOR'S DECLARATION
I hereby declare that I am the sole author of this dissertation. This is a true copy of the dissertation, including any required final revisions, as accepted by my examiners.

I authorize Ryerson University to lend this dissertation to other institutions or individuals for the purpose of scholarly research.

I further authorize Ryerson University to reproduce this dissertation by photocopying or by other means, in total or in part, at the request of other institutions or individuals for the purpose of scholarly research.
\end{abstract}

I understand that my dissertation may be made electronically available to the public. 


\title{
Title: Development of An Electromagnetic Actuator Based Translation Micromirror as A Movable Mirror in A Miniaturized FTIR Spectrometer
}

\section{Degree: Doctor of Philosophy, 2018, Yuan Xue}

\section{Mechanical and Industrial Engineering, Ryerson University}

\begin{abstract}
Miniaturized FTIR spectrometer has been developed rapidly in recent years due to the increasing demands. MEMS micromirrors have been used to replace the movable mirror system, which is the largest part in conventional FTIRs. Electromagnetic actuators are suitable to drive the micromirrors because of their relatively large quasi-static translation range and high response speed. In addition, high surface quality is required for the micromirror. However, current MEMS based micromachining technologies cannot provide satisfactory surface quality. Therefore, a translation mircomirror with a large displacement, i.e., > $120 \mu \mathrm{m}$, and a novel magnetic field pulling-force assisted bonding technology are developed to bond a high surface quality (i.e., roughness of $2 \mathrm{~nm}$ and radius curvature over $15 \mathrm{~m}$ ) mirror plate with a released microactuator using an adhesive. However, the touching points between the moving film and the substrate lead to a large starting position variation and low repeatability in operation. To solve these limitations, a repulsive force based translation micromirror utilizes a novel driving mechanism, i.e., permanent magnet ring above and electromagnet underneath the moving film, to lift and push the moving film away from the substrate for translation. As a result, the starting position of the repulsive force translating mirror is consistent and the repeatability is <
\end{abstract}


1\%. A maximum displacement of $144 \mu \mathrm{m}$ can be achieved when a $140 \mathrm{~mA}$ current is applied. To eliminate the tilt of the translation micromirror during motion, a compensation system is developed which includes the translation mirror, a correcting mirror and a reflecting mirror. The correcting micromirror corrects the tilt by rotating the same angle as the translation micromirror with its rotating axis parallel to the tilting axis. The tilt of the attractive force translation micromirror can be reduced to $0.026^{\circ}$ after compensation, so it can be used as a movable mirror in FTIRs to measure half of the midinfrared region between $13.6 \mu \mathrm{m}$ and $25 \mu \mathrm{m}$. Therefore, the electromagnetic actuator based translation micromirror with large displacement, high surface quality can be successfully used as the movable mirror in the miniaturized FTIRs with the tilt compensation system. 


\section{ACKNOWLEDGEMENTS}

I would like to thank:

* My parents for their love and countless sacrifices

* My wife, Yao, for her immense patience, emotional support and sacrifices.

* My supervisor, Dr. Siyuan He, for his encouragement, support and guidance in this research project

* Dr. Qiang Li for taking SEM measurements and photos

* Chao Ma for providing equipment in the acceleration experiment

* Chao Fan, Hui Zuo, Xuming Gao, Karl Marx, and Kenneth Tam for being great graduate friends throughout my $\mathrm{PhD}$ time at Ryerson University

* The following groups for their collaboration and financial support on this project:

$\checkmark$ Ryerson University

$\checkmark$ University of Toronto

$\checkmark$ NanoFAB in the University of Alberta

$\checkmark$ CMC Microsystems

$\checkmark$ Alcohol Countermeasure Systems (ACS) Corp 


\section{TABLE OF CONTENTS}

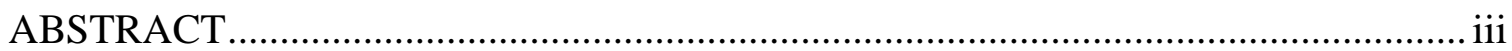

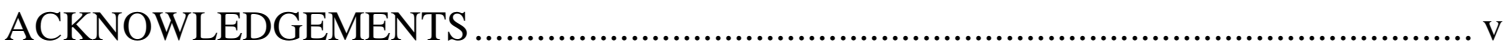

LIST OF TABLES

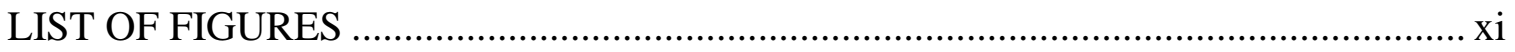

LIST OF APPENDICES .......................................................................................... xvi

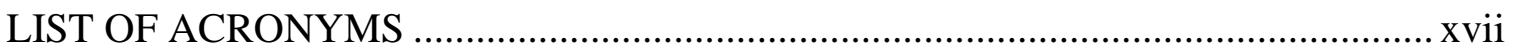

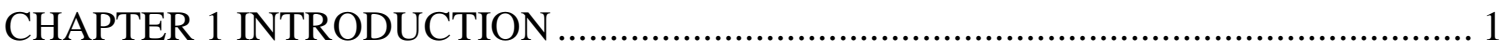

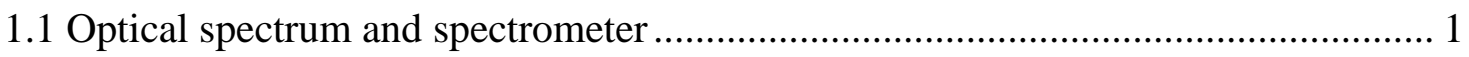

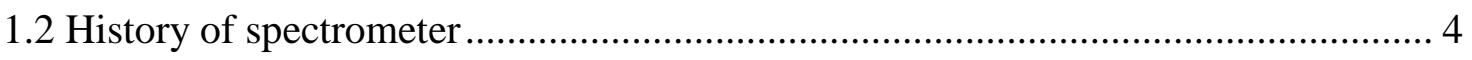

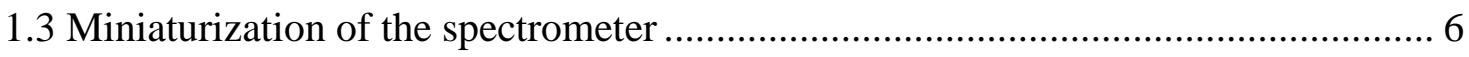

1.3.1 Miniaturization of grating based spectrometer ................................................... 6

1.3.2 Miniaturization of Michelson interferometer based FT spectrometer................ 7

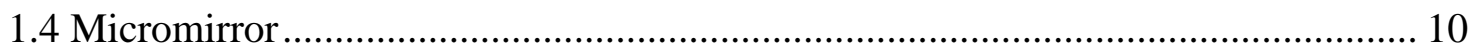

1.4.1 Micromirror actuators................................................................................ 10

1.4.2 Surface quality of the micromirror ............................................................. 16

1.4.3 Translation of the micromirror without tilting ................................................. 16

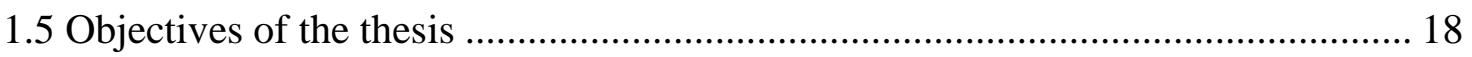

CHAPTER 2 A TRANSLATION MICROMIRROR WITH LARGE QUASI-STATIC

DISPLACEMENT AND HIGH SURFACE QUALITY ................................................. 20

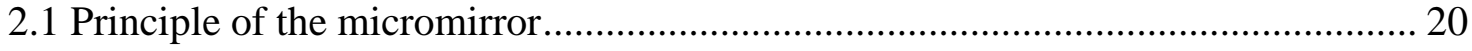

2.1.1 Micromirror structure ………………........................................................ 20

2.1.2 Raise the central ring structure ………………….......................................... 21

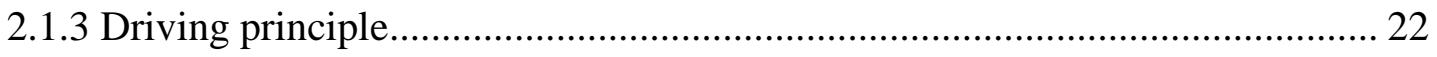

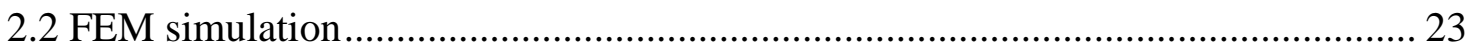




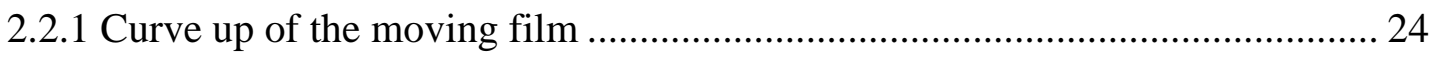

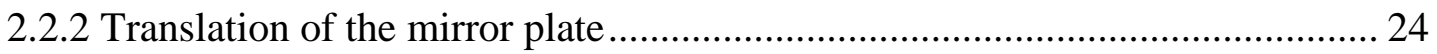

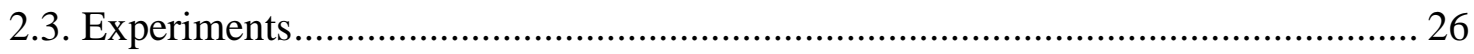

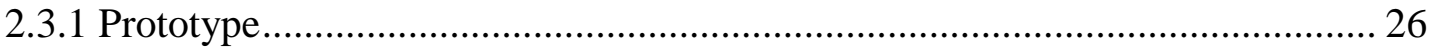

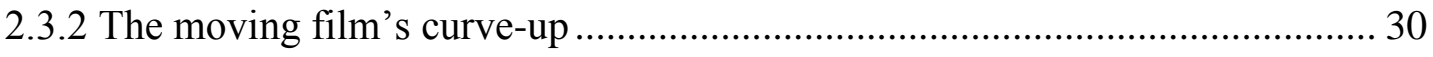

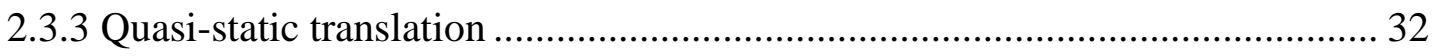

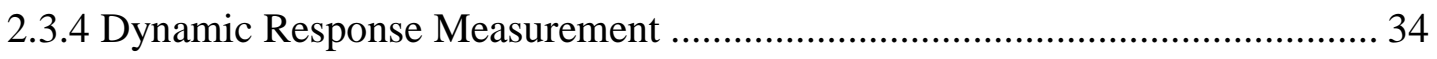

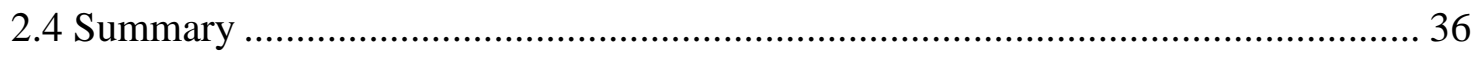

\section{CHAPTER 3 MAGNETIC FIELD ASSISTED BONDING TECHNOLOGY FOR}

RELEASED MICRO ACTUATOR AND MIRROR SURFACE ................................. 38

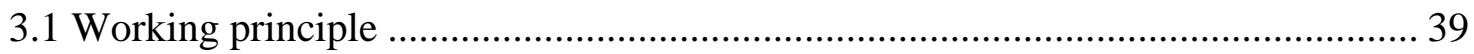

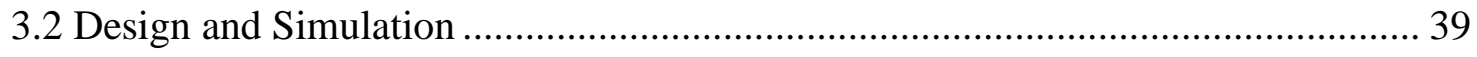

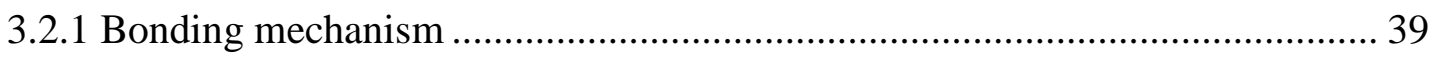

3.2.2 Simulation of distance between magnet and moving part of microactuator .... 43

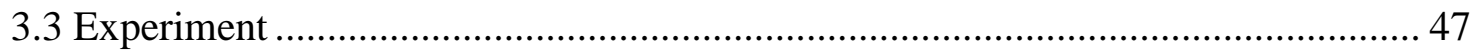

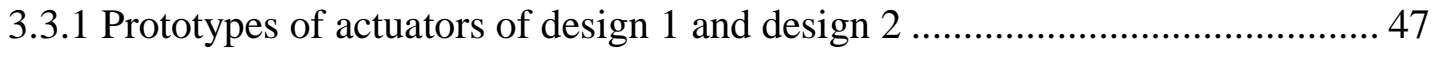

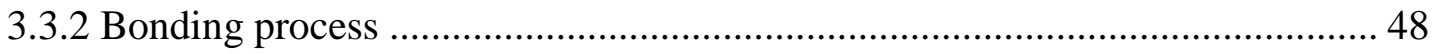

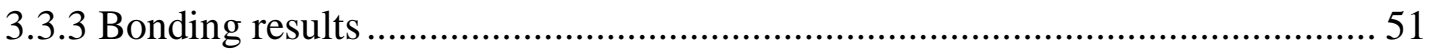

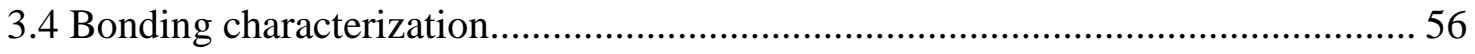

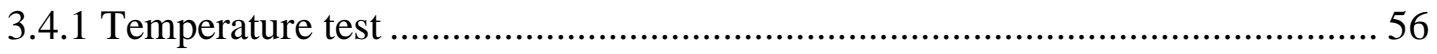

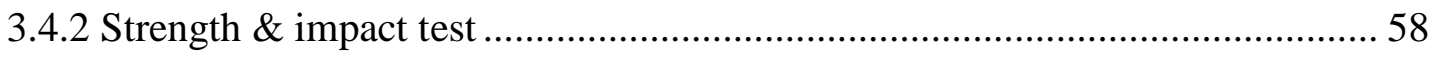

3.4.3 Performance of the bonded micromirror ................................................59

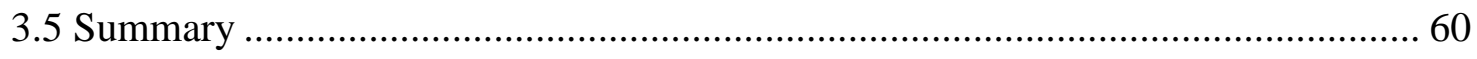

CHAPTER 4 A REPULSIVE MAGNETIC FORCE DRIVEN TRANSLATION

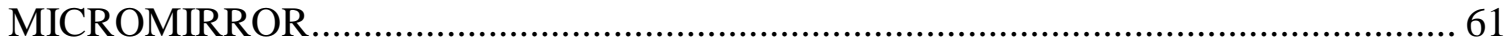

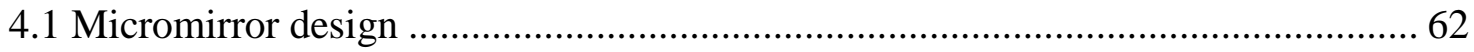




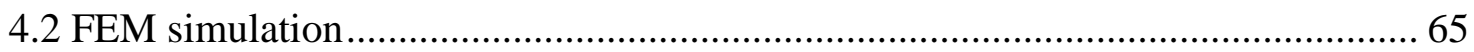

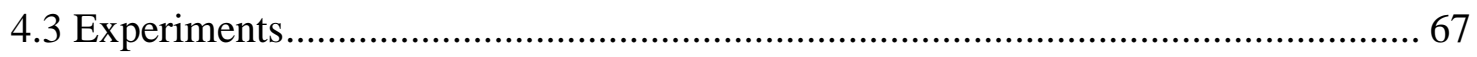

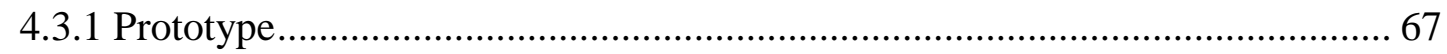

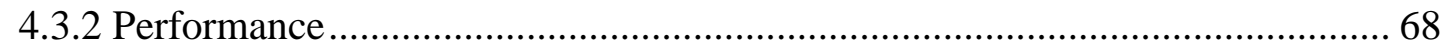

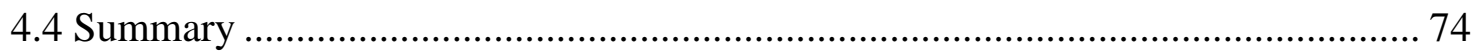

\section{CHAPTER 5 A TILT COMPENSATION SYSTEM FOR TRANSLATION}

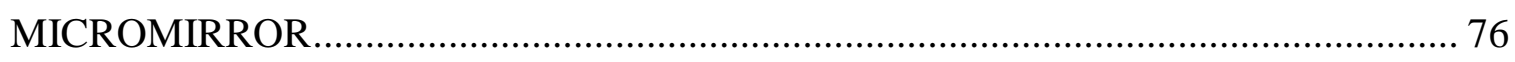

5.1 Tilt compensation system design ....................................................................... 76

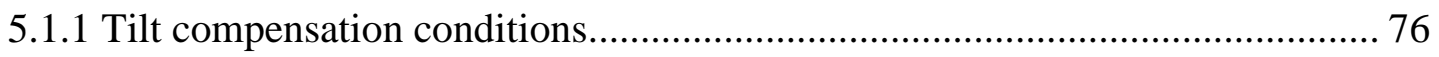

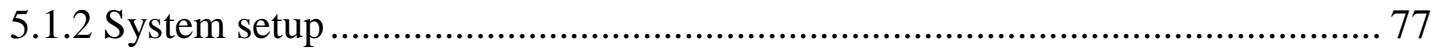

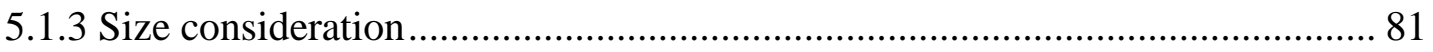

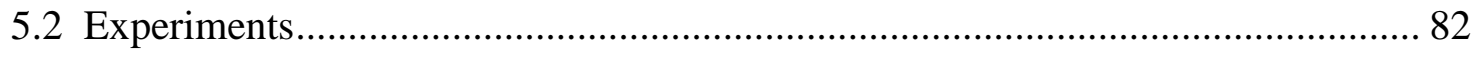

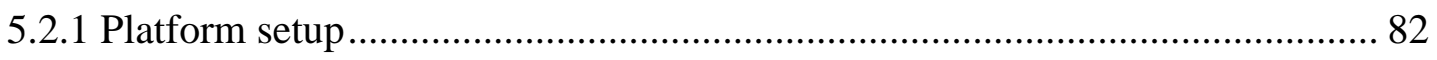

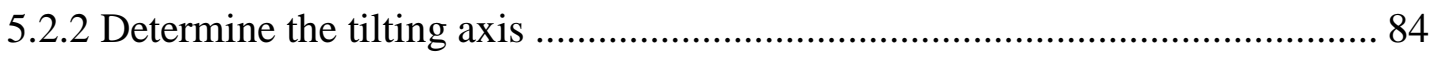

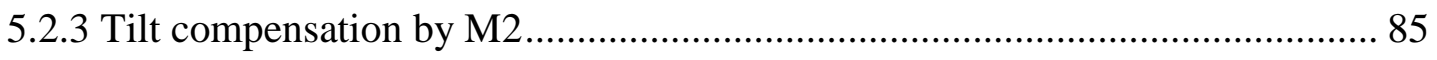

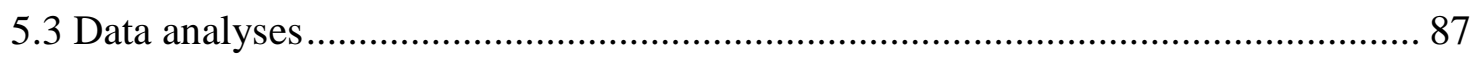

5.3.1 Error of the fringe width ................................................................................. 87

5.3.2 Interference pattern shifts caused by the variation of the titling axis ............... 89

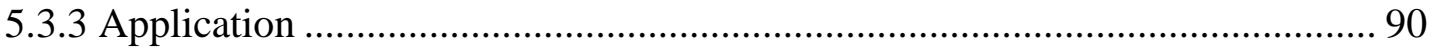

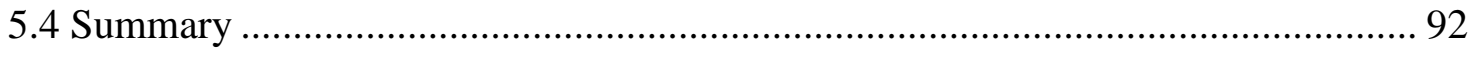

CHAPTER 6 CONCLUSIONS AND FUTURE WORK ................................................. 93

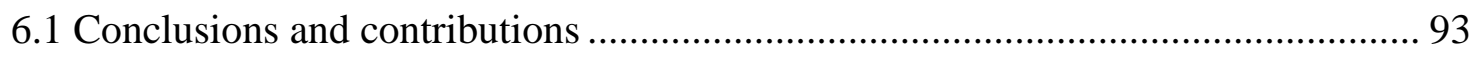

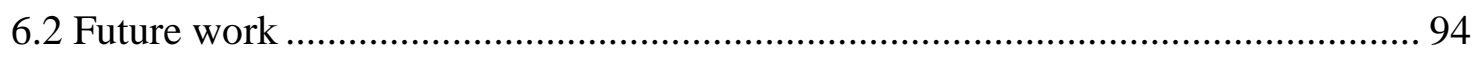

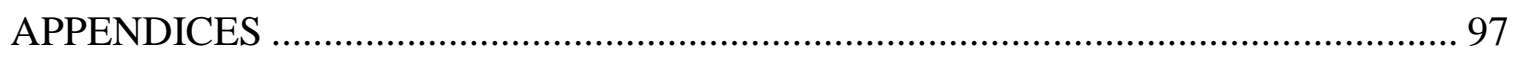

Appendix A Simulation of the moving film deformation ................................................ 97 
Appendix B Moving film moves downward when the electromagnet has a small core98

Appendix C PSD based displacement measurement.............................................. 99

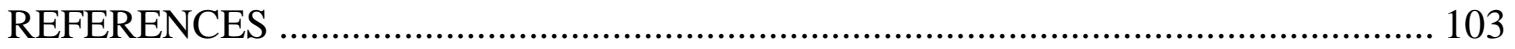




\section{LIST OF TABLES}

Table 2.1 Dimensions of the micromirror's moving part ......................................... 27

Table 2.2 Dimensions of the custom and commercial solenoid .................................. 30

Table 3.1 Dimensions of the bonding mechanism....................................................... 42

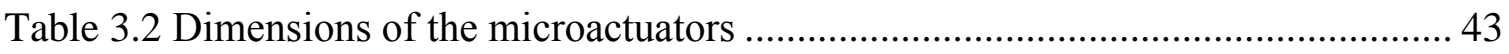

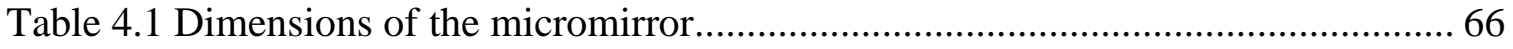

Table 4.2 Measurement result for $532 \mathrm{~nm}$ wavelength laser beam ............................... 74 


\section{LIST OF FIGURES}

Figure 1.1 Grating (1) and prism (2) spectrometers [2] .......................................... 2

Figure 1.2 Diagram of Fabry-Perot (F-P) spectrometer............................................ 2

Figure 1.3 Diagram of FT spectrometer ................................................................ 3

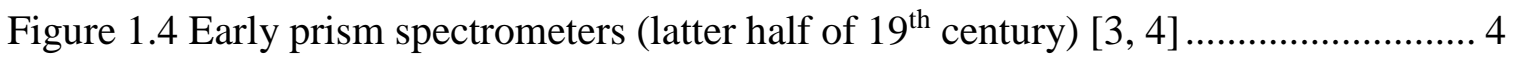

Figure 1.5 Grating spectrometers. (a) Coleman Junior II Spectrophotometer (1960); (b) Shimadzu IR-27G double beam self-recording spectrophotometers $(1965,130 \mathrm{~kg})$ [10] .. 5

Figure 1.6 FT spectrometer - Shimadzu FTIR-4000 (1984) [13] .................................. 6

Figure 1.7 Miniaturized grating based spectrometer. (a) Fiber Optic Spectrometer - S2000

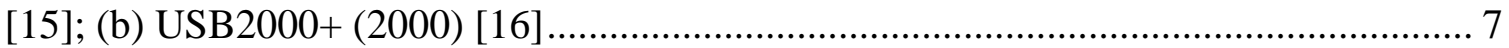

Figure 1.8 Miniaturized LIGA based FTIRs (2003) [17] ............................................ 8

Figure 1.9 MEMS micromirror based FTIRs [18] ............................................... 8

Figure 1.10 Structure of the movable mirror system in the conventional actuator. (a) Miniscale linear air bearing [19]; (b) Mini-scale rolling elements linear mechanical bearing [20]

Figure 1.11 Electrostatic actuator working at resonant frequency [68]

12

Figure 1.12 Two types of electro-thermal actuators. (a) Top view of a lateral driven thermal

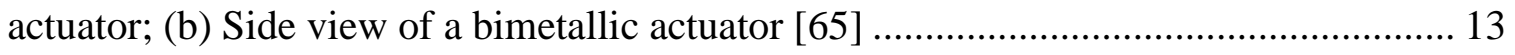

Figure 1.13 Thermal actuator based FT spectrometer [47] ......................................... 13

Figure 1.14 Lorontz force and Magnetostatic force. (a) Wire forced by Lorontz force after current appiled; (b) A piece of iron forced in the magnetic field .................................. 14

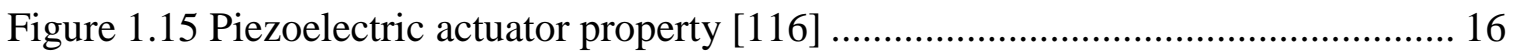

Figure 1.16 Tilt exists in conventional Michelson interferometer................................ 17

Figure 2.1 Concept of the micromirror. (a) Actuator moving film plus substrate; (b)

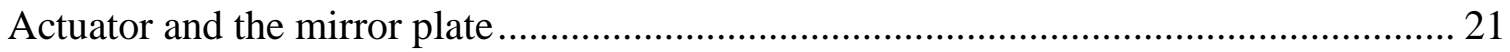

Figure 2.2 Mirrormirror with no mirror plate bonded curves up through the curve-up mechanism and negative residual stress gradient in the nickel film. (a) Micromirror before release; (b) Micromirror after release 22 Figure 2.3 Micromirror principle. (a) Moving film in the magnetic field generated by the solenoid; (b) Magnetic field generated by the solenoid; (c) Central ring structure under the magnetic field 23 
Figure 2.4 Combining simulation of magnetic field and mechanical deformation. (a) Magnetic field simulation to obtain the force on the moving film; (b) Simulation of the moving film deformation under the residual stress gradient, mirror plate weight and the

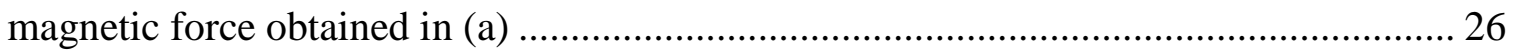

Figure 2.5 Prototype of the translation micromirror. (a) Moving film (nickel film); (b) Moving film bonded with the mirror plate; (c) Mirror plate \& moving film chip; (d) Assembled micromirror with self-designed solenoid; (e) Assembled micromirror with

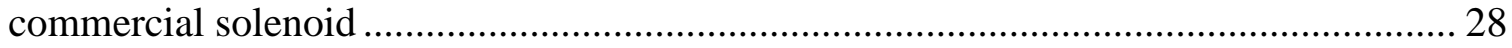

Figure 2.6 Surface quality of mirror plate. (a) Roughness; (b) Curvature....................... 29

Figure 2.7 The reflectance of the mirror plate (input power 1.03W) ........................... 29

Figure 2.8 Prototypes of the moving film and the mirror plate. (a) Curve-up mechanism;

(b) Curve-up mechanism with mirror plate 31

Figure 2.9 Static translation measurement. (a) Protection cover \& mounting holder; (b) Static performance measured using Zygo profiler. 33

Figure 2.10 Static performance of the micromirror. (a) Translations of experiment and simulation; (b) Tilting angle of the micromirror. 33

Figure 2.11 Dynamic measurement setup. 34 Figure 2.12 Concept of dynamic translation measurement. (a) Laser spot movement on PSD surface caused by micromirror translation; (b) Laser spot movement on PSD surface caused constructively by the translation and tilting of the micromirror 35 Figure 2.13 Settling time of the micromirror. (a) Settling time of micromirror downward; (b) Settling time of micromirror upward 37

Figure 3.1 Released miroactuator and mirror plate before bonding (left); Conventional bonding mechanical using compression force (top right); and Novel bonding mechanism using magnetic field pulling-force (bottom right)

Figure 3.2 The bonding mechanisms. (a) Bonding mechanism with the video camera under the mirror plate; (b) Bonding mechanism with a stage and magnet replacing the video camera; (c) Zoom in image of the alignment structure 41

Figure 3.3 Microactuators for bonding. (a) Design 1; (b) Design 2 42 
Figure 3.4 Magnetic and deformation simulations for design 1 and design 2. (a) Distribution of magnetic field in design 1 and design 2; (b) Displacement ( $\mathrm{D}_{\text {actuator_ring }}$ ) of design 1 and design 2

Figure 3.5 Stress in designs 1 and 2 when displacement is $\mathrm{D}_{0}$. (a) Maximum Von Mises stress of $107 \mathrm{MPa}$ in design 1; (b) Maximum Von Mises stress of $115 \mathrm{MPa}$ in design 2. 46 Figure 3.6 Measuerment under 3D optical profiler. (a) Measurement using low magnification lens; (b) Measurement using high magnification lens ............................. 48

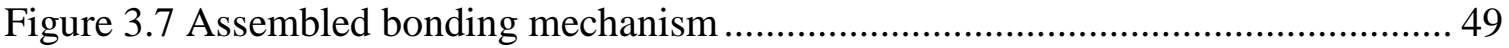
Figure 3.8 Bonding process. (a) Adhesive is applied to the backside of the mirror plate; (b) The microactuator is positioned on the aluminum cylinder; (c) The microactutor is put in the chip holder and fixed on the alignment structure; (d) The central ring of the moving part and the mirror plate are laterally aligned to ensure the mirror plate on the center of the ring by the 3-axis stage and monitored by a video camera; (e) The permanent magnet is raised up by a lab jack to pull the moving part of the microactuator down to touch the mirror plate; (f) After curing the adhesive, the microactuator with the mirror plate bonded

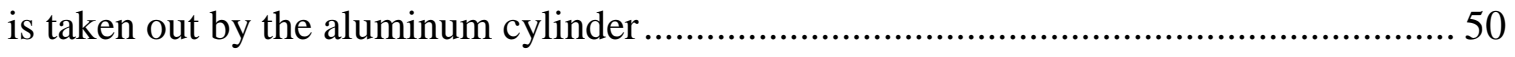

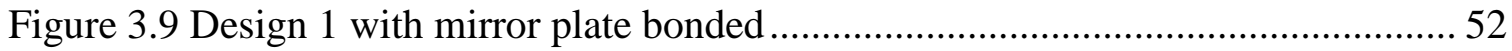
Figure 3.10 Cross section profiles along lines AB (as shown in Figure 3.6) before and after bonding 52

Figure 3.11 Measurement result of design 1 after bonding. (a) Points measured on design

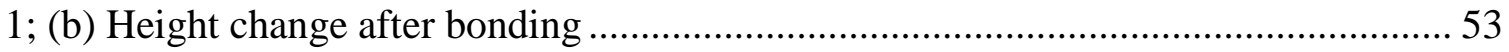

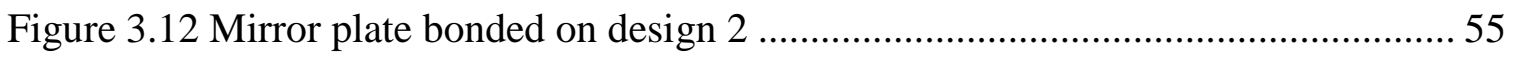

Figure 3.13 Cross section profiles (lines $\mathrm{AB}$ in Figure 3.12) of design 2 before and after bonding 55

Figure 3.14 Temperature raised by increasing the power of the laser beam ...................56

Figure 3.15 Test platforms. (a) High power laser test; (b) Heating test ......................... 57 Figure 3.16 (a) Setup for strength test using the shaker; (b) Setup for impact test; (c) Result of impact test. 58

Figure 3.17 Displacement and tilting angle of the micromirror under various currents. (a) Displacement of micromirror; (b) Tilting angle of micromirror .59 
Figure 4.1 Concept of the micromirror. (a) Moving film of the micromirror; (b) The fixed and moving part of the micromirror.

Figure 4.2 Lower corners of each trapezoidal plate touching on the substrate before the permanent magnet is placed 63

Figure 4.3 Working princinple of mivromirror. (a) Moving part pre-lifted by the permanent magnet; (b) Moving part moves upward (current applied) 64

Figure 4.4 3-D model of a quarter of the micromirror. 66

Figure 4.5 Stress simulation using CoventorWare 67 Figure 4.6 Micromirror before and after assembly. (a) Micromirror before the assembly; (b) Assembled micromirror..... 68

Figure 4.7 SEM images show the corners touching on the substrate before assembly. (a) Prototype of the moving part before assembly; (b) Trapezoid plate lower corners touching on the substrate

Figure 4.8 The lower corners of trapezoidal plate are separated from substrate in the permanent magnet and repulsive magnetic force translation micromirror. (a) The lower corner of trapezoidal plate touching on the substrate before the permanent magnet and the electromagnet assembled; (b) The lower corner of trapezoidal plate separated from the substrate after assembling the permanent magnet before applying current through the electromagnet; (c) The lower corner of trapezoidal plate moves upward after current is applied to the electromagnet (This figure shows the example of applying low current).. 70 Figure 4.9 Micromirror translation in static model. (a) Translations in experiment and simulation; (b) Tilting angle of the micromirror.

Figure 4.10 Driving circuit for micromirror [153]. 72 Figure 4.11 Fringes patterns appeared when the micromirror is at different displacement. (a) Michelson interferometer with designed movable micromirror; (b) Straight fringes patterns on the screen when the moveable micromirror travels $7.2 \mu \mathrm{m}$ (the middle one) and $29.5 \mu \mathrm{m}$ (the right one) from the original position (the left one). 73 Figure 5.1 The tilt compensation system. (a) When M3 angled with the fixed mirror; (b) when M3 is perpendicular to the fixed mirror. 78 Figure 5.2 Different shapes of interference fringes generated by a Michelson interferometer. a) Circular fringes; b) Straight fringes [155] 79 
Figure 5.3 Determination of tilting axis. a) Micromirror tavels on the Y-axis with tilt along a rotation axis other than the $\mathrm{Z}$-axis; b) with tilting along the Z-axis 79 Figure 5.4 Packaged tilt compensation system with a re-designed assembly part for repulsive magnetic force translation micromirror. 81 Figure 5.5 Michelson interferometer with a tilt compensation system; (a) Top view of the experimental platform; (b) Detailed view of tilt compensiton system; (c) Detailed view of the movable mirror M1; (d) Detailed view of the 1D rotation mirror M2. 83 Figure 5.6 Determine the tilting axis using the PSD and the oscilloscope. (a) Experiment setup; (b) The minimum voltage $(<10 \mathrm{mV})$ is observed when $\mathrm{M} 1$ rotates $72^{\circ}$ and the displacement is around $100 \mu \mathrm{m}$ 84 Figure 5.7 Interference pattern on the screen. a) $1^{\text {st }}$ reference fringes; b) Spots misaligned under $30 \mu \mathrm{m}$ displacement without compensation; c) Interference pattern when M1 travels $30 \mu \mathrm{m}$ with compensation; d) $2^{\text {nd }}$ reference fringes; e) Only the spot from the fixed mirror is shown on the screen due to no compensation; f) Interference pattern when M1 travels

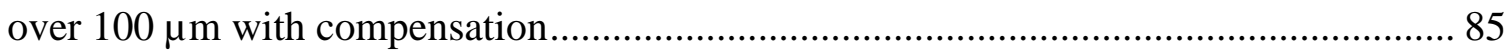

Figure 5.8 Interference firinge change due to the tilt of M1. 87

Figure 5.9 Fringe width on the same interference pattern. (a) Tolerable error range (14 mm $\pm 1 \mathrm{~mm}$ ); (b) Out of the tolerable range because the line is in the mid of the dark (left) or bright (right) stripe 87

Figure 5.10 Angle difference $\Delta \theta$. 88

Figure 5.11 Tilt analysis. (a) Top view of the compensation system; (b) Side view of the interference pattern on the screen 90

Figure 5.12 The movable mirror tilts $\beta$ with displacement. 91 Figure 5.13 Spectral Range [166] 91 


\section{LIST OF APPENDICES}

Appendix A Simulation of the moving film deformation..........................97

Appendix B Moving film moves downward when the electromagnet has a small core.....98

Appendix C PSD based displacement measurement................................. 99 


\section{LIST OF ACRONYMS}

AAS Atomic absorption spectroscopy

CTE Coefficients of thermal expansion

FEM Finite element analysis

FPCB Flexible printed circuit board

FT Fourier transform

FTIRs Fourier transform infrared spectrometer

LIGA Lithographie, Galvanoformung and Abformung (Lithography, Electroplating, and Molding)

MEMS Microelectromechanical system

MetalMUMPs Metal multi-user MEMS process

MMF Magneto motive force

MUMPs Multi-user MEMS process

NIR Near infrared light

OPD Optical path distance

PI Personal instrument

PSD Position sensing detector

PTFE Polytetrafluoroethylene

SEM Scanning electron microscope

SUMMiT Sandia Ultra-planar, Multi-level MEMS Technology

UV Ultra-light

VIS Visible light 


\section{CHAPTER 1 INTRODUCTION}

\subsection{Optical spectrum and spectrometer}

The optical spectrum is a part of the electromagnetic spectrum. The study of optical spectrum started in the $17^{\text {th }}$ century when Isaac Newton observed the phenomenon that a series of colors ranging from red to purple are generated after the natural light was dispersed by a prism. The optical spectrum is based on the theory that when light is projected to a matter, the atom of the matter absorbs the corresponding energy from the light, which leads to the change of light's wavelength. Spectrometer, or spectrograph, is the device to record the intensity of the light passing through a sample and obtain the spectrum. The components of the sample are then determined by using spectrum analysis. As an important optical instrument, spectrometer has been widely employed in various fields including agriculture, food safety, biology, medicine, petrochemistry, astronomy and the environment.

The spectrometer has three major parts which are the light source, dispersion system [1] and detector. The dispersion system plays an important part because spectral measurement range, dispersive power and resolution are dependent on it. Basing on the different working principles of the dispersion system, the spectrometers can be divided into four types:

1) Material dispersion spectrometer - It utilizes the principle that the lights with different frequencies travel at different speeds in the same medium, so their refractive indexes are different. An example of the material dispersion spectrometer is the prism spectrometer, which uses a prism as the dispersive element, as shown in the lower left corner of Figure 1.1. The light is dispersed by the prism when propagates through it and the refraction angle depends on the refractive index of the prism's material and the wavelength of the light.

2) Diffraction grating spectrometer - This type of spectrometer uses the diffraction grating as the dispersive element. Because different wavelengths lead to different diffraction angles even if the incidence angle is same, the incident beam is separated and diffracted after passing through multiple splits on the grate and becomes multiple lights travelling in different directions, as shown in the upper left corner of Figure 1.1. 


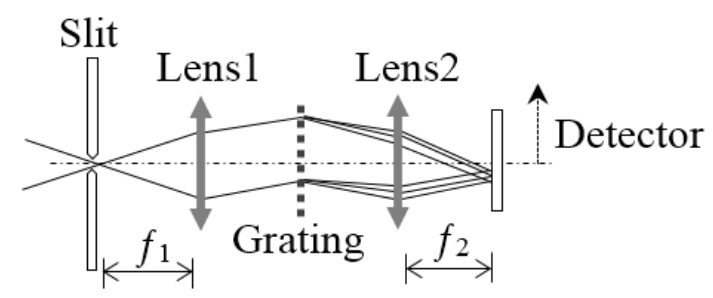

Diagram (1) of grating spectrometer

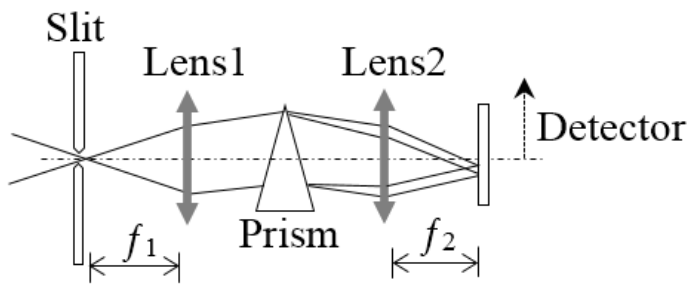

Diagram (2) of prism spectrometer

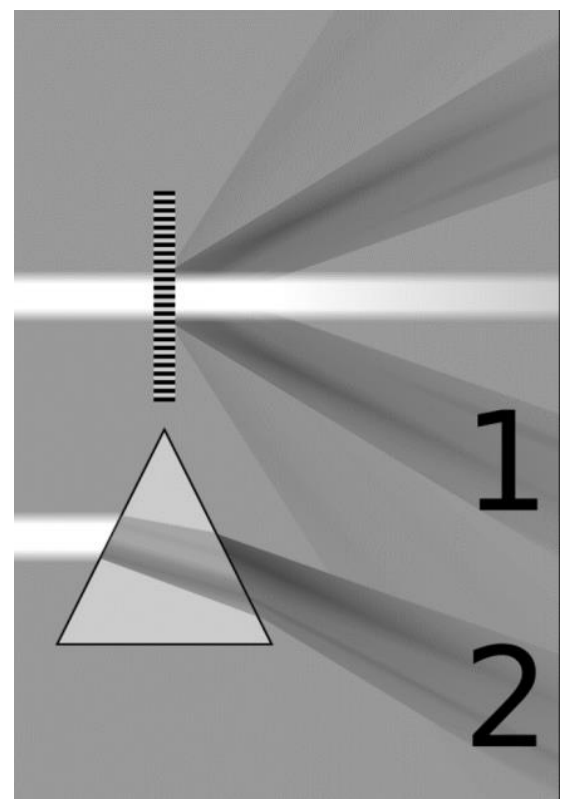

Figure 1.1 Grating (1) and prism (2) spectrometers [2]

3) Fabry-Perot (F-P) spectrometer - As shown in Figure 1.2, this spectrometer is composed of a pair of separated glass with high surface quality. The surfaces between two glass are highly reflective, so the incident beam is reflected multiple times between the pair and generates multiple transmitted beams. Because the optical paths of these reflected beams are different, the beams interfere with each other to generate the interferogram on the screen.

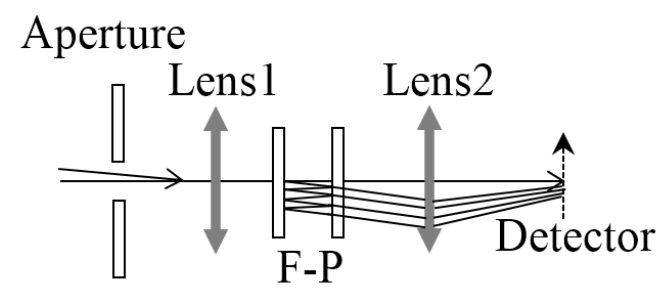

Figure 1.2 Diagram of Fabry-Perot (F-P) spectrometer

4) FT spectrometer - It uses an interferometer as the disperse system. The incident beam produces an interferogram after passing through the interferometer, then a spectrogram is obtained after the Fourier transform of the interferogram. As shown in Figure 1.3, the Michelson interferometer is most commonly used in FT spectrometer. 


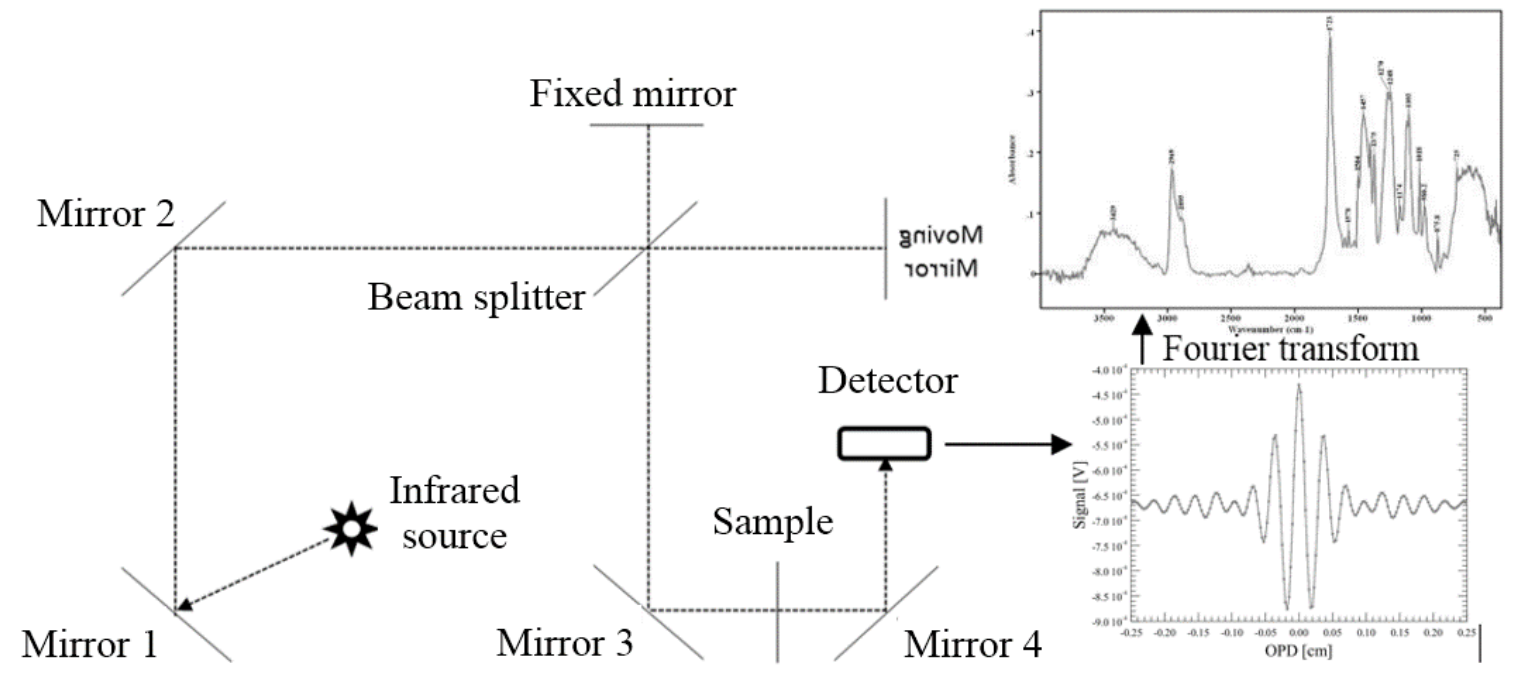

Figure 1.3 Diagram of FT spectrometer

Different dispersion systems have different characteristics. The prism system has relatively low performance, but its structure is simple and it is the only spectrometer generating spectrum without superposition. Therefore it is suitable to work as the predisperser in a composite spectrometer. The F-P spectrometer has high resolution and large aperture (large optical flux), but its free spectral range [3] is very small and the structure and assembly are relatively complex, so it is commonly used in laboratories for the research of ultra-fine structures.

The grating dispersion system is one of the most popular spectrometers. It has such advantages as wide spectral range in theory, simple structure and relatively low requirements on assembly. In fact, due to the manufacture limitation and the high cost, the measurement range of the grating spectrometer is concentrated on UV (325-380 nm) [4], VIS (380-650 nm) [5] and part of NIR (650-835 nm) [6] whose wavelength is below $1 \mu \mathrm{m}$. Therefore, the grating spectrometer is mainly used in geological prospecting and atmosphere measurement, because the wavelengths of inorganic substances are in this range. The FT dispersion system is also popular. Its advantages include higher resolution and higher signal-to-noise ratio by compared to the grating spectrometer with the same aperture size. As the result, its overall performance is higher. However, it is limited by the performance of the movable mirror of the interferometer, such as the displacement, steps and tilt. It is mainly used in infrared measurement of wavelength greater than 0.75 
$\mu \mathrm{m}$, most of which are organic substances, so it is popularly used in food safety, biology and medicine.

\subsection{History of spectrometer}

The first prism spectrometer was made and improved by Gustav Kirchhof (1824-1887) and Robert Bunsen (1811-1899) in 1859, as shown in Figure 1.4. In the next 100 years since then, the prism spectrometer, as the first generation spectrometer [7][8], was mainly used in the laboratories to observe the optical phenomenon and prove optical theories. It contributed greatly to the early development of spectroscopy.

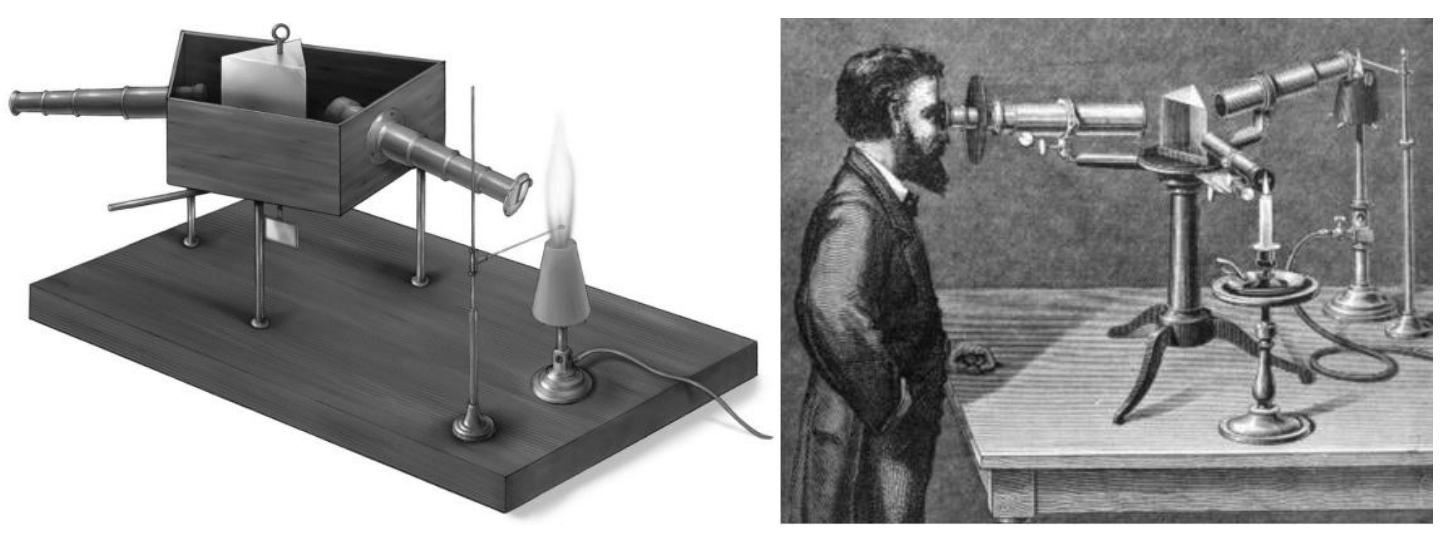

Figure 1.4 Early prism spectrometers (latter half of $19^{\text {th }}$ century) $[3,4]$

Since 1960s, the industrial and electrical technologies had been advanced, especially the grating ruling and replica technology and AAS [9], therefore the prism was replaced by the grating to be used as the dispersion element in the second generation spectrometer [10], as shown in Figure 1.5. Compared with the prism spectrometer, the grating spectrometer can make more precise qualitative and quantitative analysis and measure wider wave bands, including UV, VS and even part of NIR.

Both the prism and the grating spectrometers are dispersion systems based on the diffraction. By applying a FT to the interferogram, the spectrum was obtained by Fellgett in 1958. It proved multiple advantages of the FT spectrometer, such as high signal-tonoise ratio [11] and better resolution of the spectrum [12]. Since then, interference spectrometers have hade significant improvements with the effort from Jacquinot, Fellgett, Connes P, and Strong's Group. With the development of photoelectric technology and the rapid advancement of computer science, FT spectrometry has become 
the third generation spectrometer since 1980s. Michelson interferometer based FTIRs is used as the most popular type until now. Figure 1.6 shows Shimadzu FTIR-4000, which was produced in 1984. This FTIRs has a high recording speed and storage using multiple CPUs and a floppy disk as memory and uses an air bearing mechanism to drive the movable mirror smoothly.

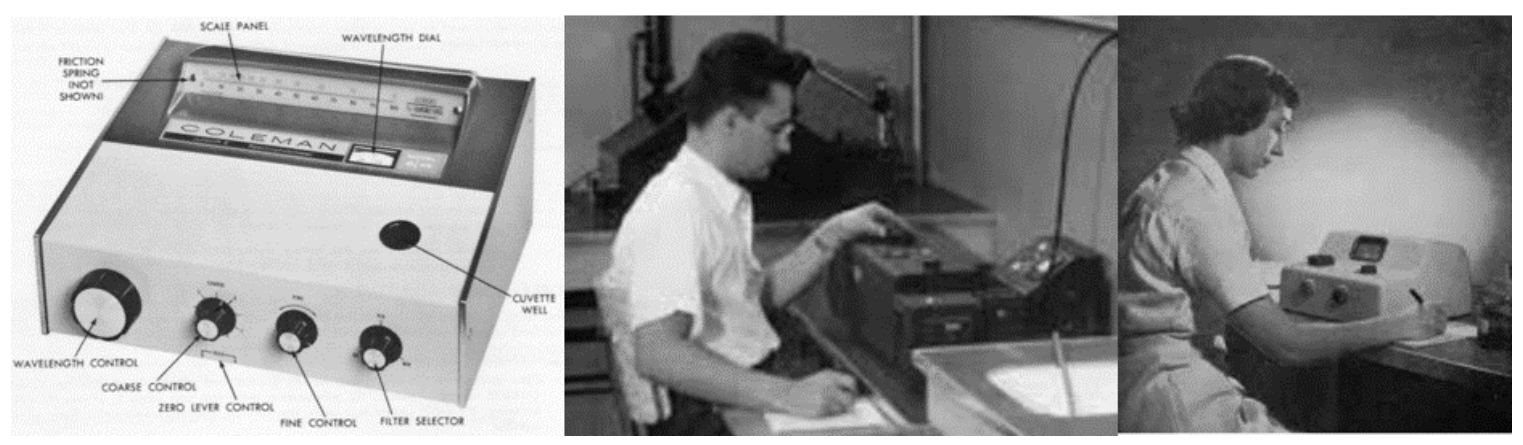

(a)

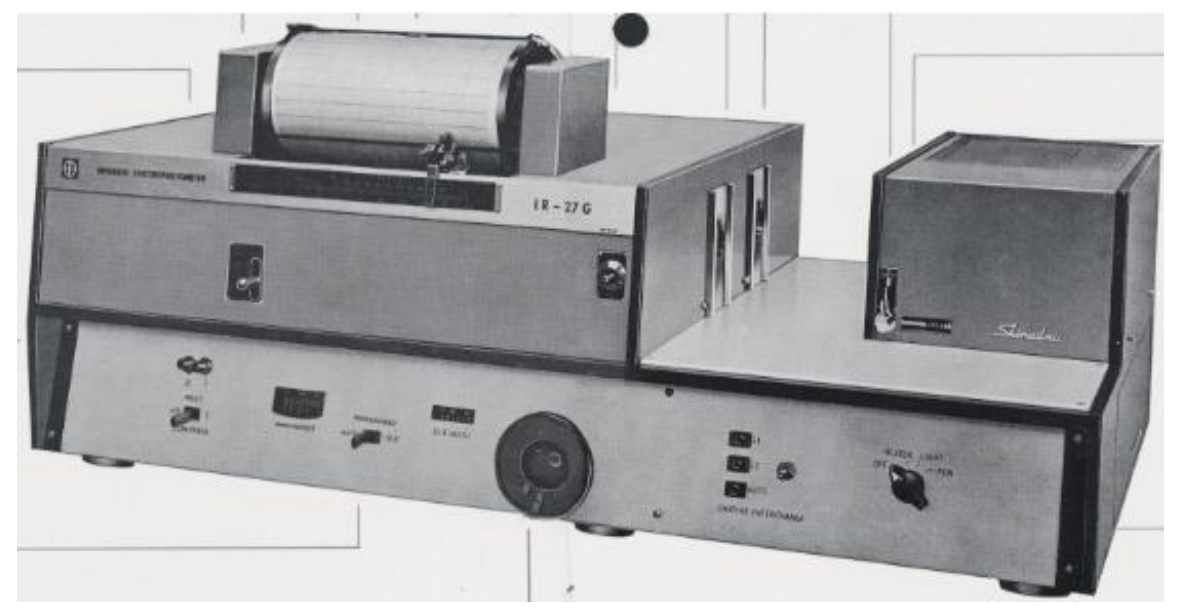

(b)

Figure 1.5 Grating spectrometers. (a) Coleman Junior II Spectrophotometer (1960); (b) Shimadzu IR-27G double beam self-recording spectrophotometers $(1965,130 \mathrm{~kg})$ [10] 


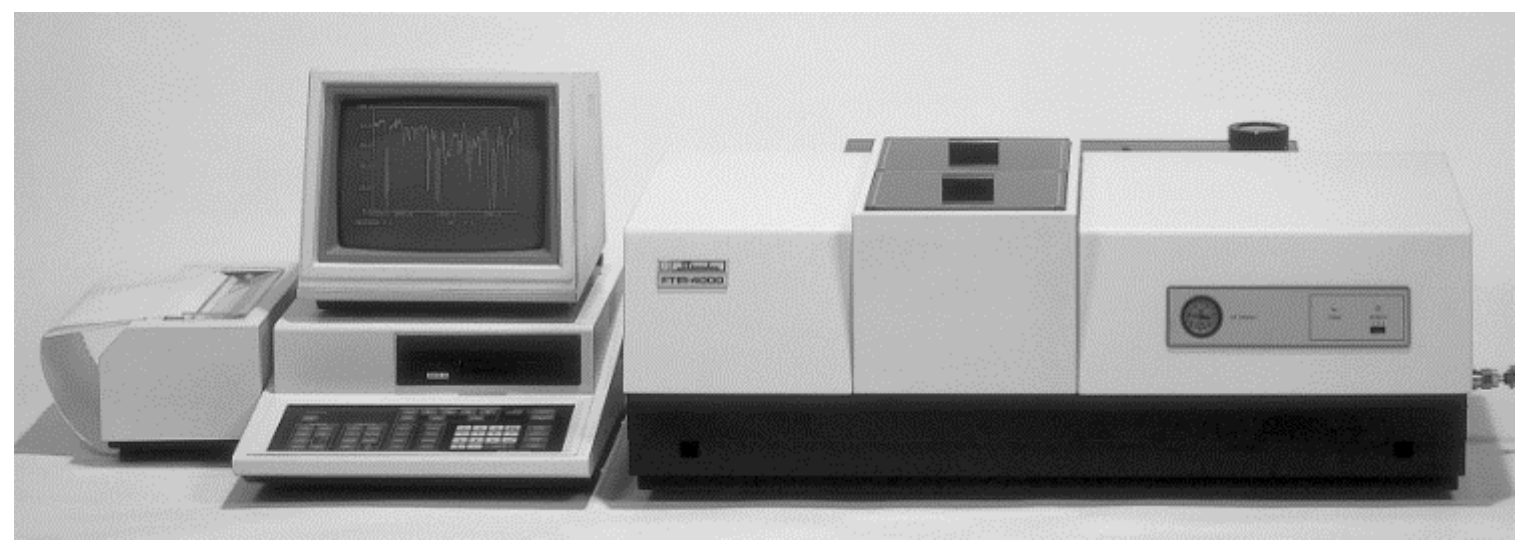

Figure 1.6 FT spectrometer - Shimadzu FTIR-4000 (1984) [13]

\subsection{Miniaturization of the spectrometer}

The conventional spectrometers were large, expensive and sensitive to the working environment, such as temperature, humidity, shock, etc., so they could only be operated by professional operators. Therefore, there have been more demands for miniaturized portable spectrometers which can be deployed on-site since the end of last century. For example, all of the product line inspection equipment, battlefield (radiation, chemical, biochemical weapon) detection instruments and PI require the spectrometer to have such features as reliable, easy to operate, small volume, low cost and capable to provide specific functionalities. With the higher demands on life qualities, human health and food safety, PI becomes one of the most important research areas in spectroscopy. In recent years, new spectrometers have been developed and deployed as personal blood glucose detectors, urine analyzers, water quality analyzers, dangerous gas $(\mathrm{CO}$, formaldehyde, etc.) monitors [11], medicine efficacy monitors, etc. It is predictable that miniaturized portable infrared spectrometers will be more popular in the following years.

\subsubsection{Miniaturization of grating based spectrometer}

Since the end of last century, the miniaturization of spectrometer has become a popular research field. The first miniaturized spectrometer, the Fiber Optic Spectrometer S1000, was successfully developed by Dr. Mike Morris, the founder of Ocean Optical, in 1992 [14]. Its improved model S2000 [15], shown in Figure 1.7a, became the company's leading product. The dimension of the master part (no fiber or analysis computer) is only $14.3 \mathrm{~cm} \times 10.4 \mathrm{~cm} \times 4 \mathrm{~cm}(\mathrm{LWH})$ and its weight is only $200 \mathrm{~g}$, which makes the spectrometer able to work outside laboratories. At the beginning of this century, a smaller 
model USB2000+ [16], shown in Figure 1.7b, was developed and, which is only $8.9 \mathrm{~cm}$ x $6.3 \mathrm{~cm} \mathrm{x} 3.4 \mathrm{~cm}$ in LWH and weights $190 \mathrm{~g}$ for the master part. However, since it uses grating as the diversion system, it can only measure the range of spectrum between UV and VIS. So its applications are only focused on metal inspection, online detecting and some inorganic detection applications.

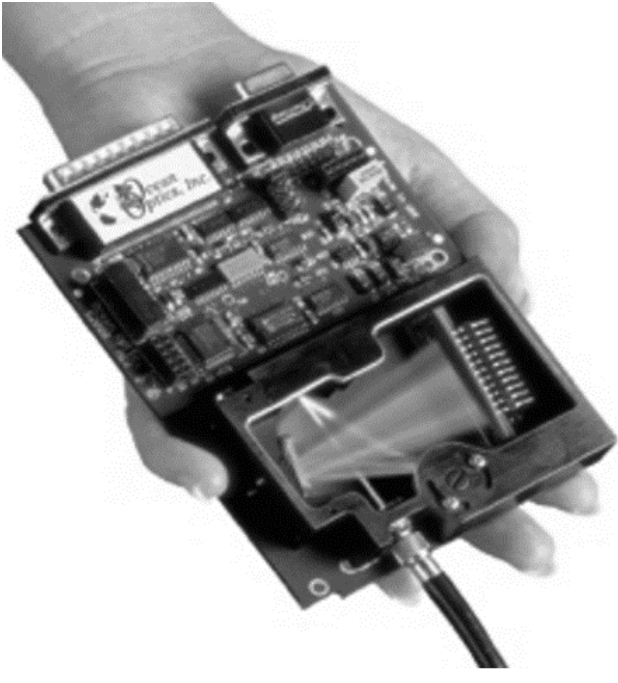

(a)

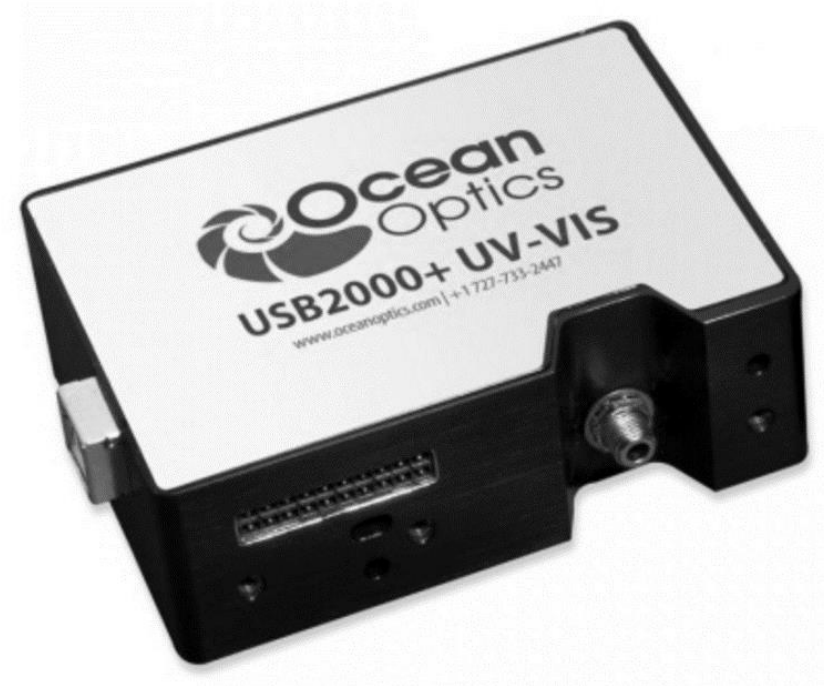

(b)

Figure 1.7 Miniaturized grating based spectrometer. (a) Fiber Optic Spectrometer - S2000

$$
\text { [15]; (b) USB2000+ (2000) [16] }
$$

\subsubsection{Miniaturization of Michelson interferometer based FT spectrometer}

An infrared spectrometer is needed as the result of the requirements on the measurement of the organic substances, e.g. alcohol, $\mathrm{CO}$, etc., which have strong absorbance in the mid-infrared region [11]. FTIRs, as the most widely used infrared spectrometer, also needs to be miniaturized. Based on its working principle, a beam is split into two parts by the beam splitter - one is injected to the fixed mirror and the other is injected to the movable mirror - then both are reflected back to the beam splitter to be combined. The interference fringes are formed when two parts of the beam are superposed and the strengths are detected by the photodetector, then the corresponding spectrum is generated after a FT by computer. Therefore, the Michelson interferometer based FT spectrometer is composed of light source, beam splitter, fixed mirror, movable mirror, photodetector, lens, fibre, etc. Due to the high integration, MEMS technology, which integrates electric, mechanics, optical sensors and control circuit into a chip, has 
been popularly accepted as a solution of miniaturized FT spectrometer and many products have been developed as a result.

At the beginning of this century, one type of the MEMS based miniaturized spectrometer integrates all components, except for the laser source, onto a chip. As shown in Figure 1.8, a miniaturized FT spectrometer was designed by Christian Solf basing on LIGA, a fabrication technology used to create high-aspect-ratio microstructures [17]. The actuator of the movable mirror and the frames of the components are fabricated on a piece of $11.5 \mathrm{~mm} \times 9.4 \mathrm{~mm}$ chip. After the chip is released, all the components, such as mirrors, beam splitter, detector and lens, are assembled on it to produce a miniaturized FT spectrometer. This system saves much space due to the high integration, but such shortcomings as the high cost and disability to adapt to the environment limit its development.

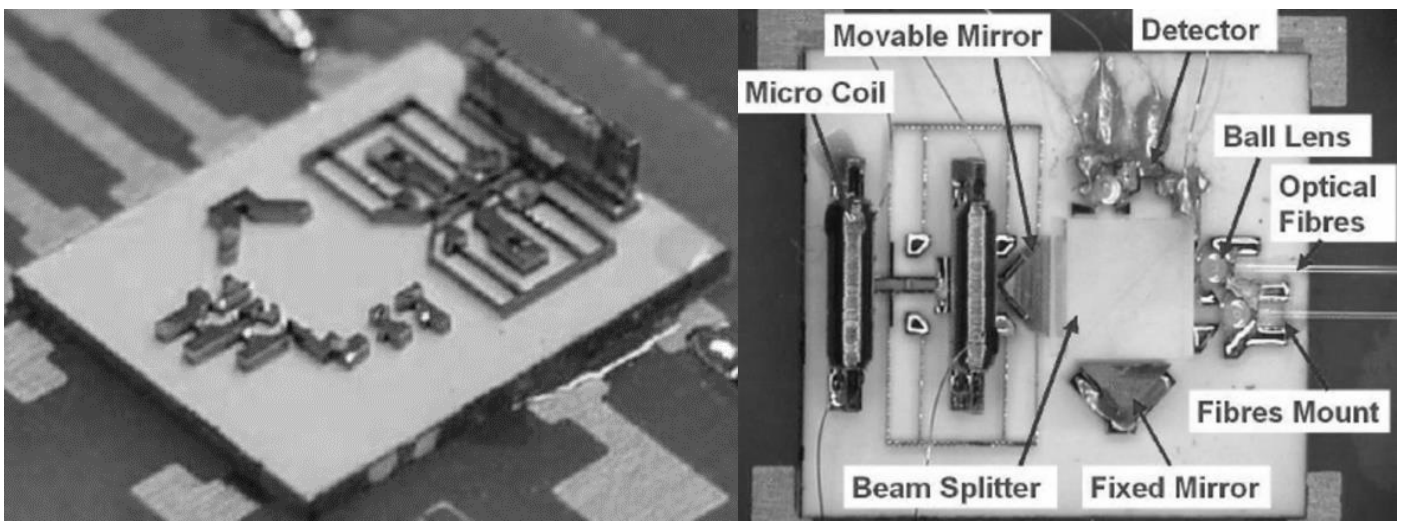

Figure 1.8 Miniaturized LIGA based FTIRs (2003) [17]
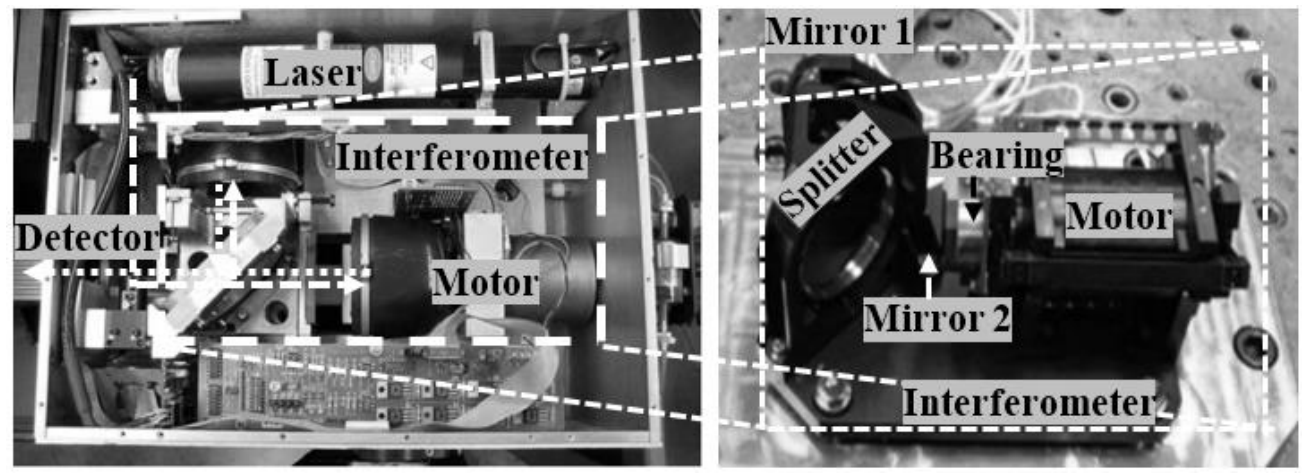

Figure 1.9 MEMS micromirror based FTIRs [18] 

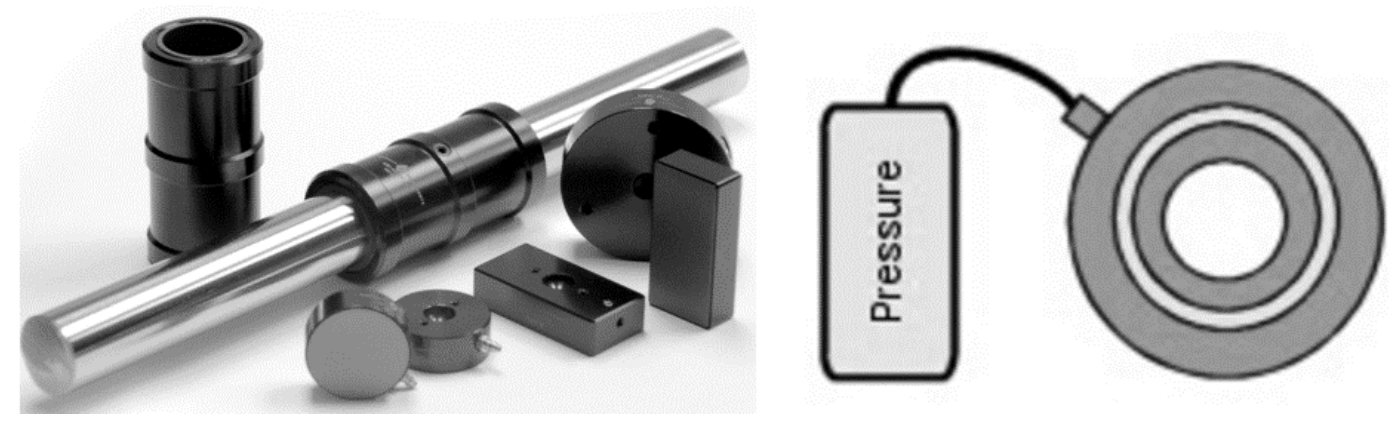

(a)
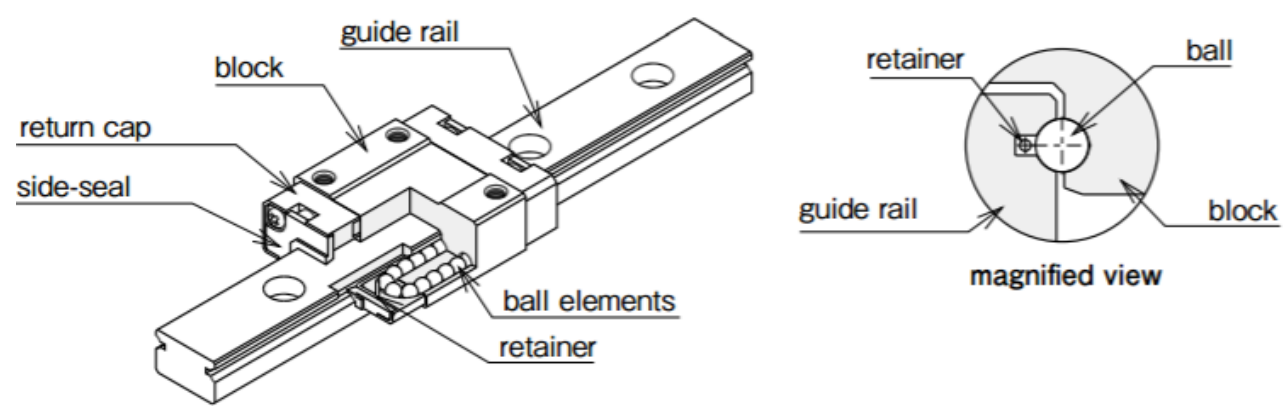

(b)

Figure 1.10 Structure of the movable mirror system in the conventional actuator. (a) Mini-scale linear air bearing [19]; (b) Mini-scale rolling elements linear mechanical bearing [20]

Another solution of the miniaturized FT spectrometer uses MEMS micromirror to replace the movable mirror system in the conventional Michelson interferometer, which are the largest part including motor, bearing and the mirror, as shown in Figure 1.9. In order to avoid the precision being impaired by the friction between the moving block and the guide rail, the bearing is made of a linear air bearing and mechanical bearing containing rolling elements as shown in Figure 1.10. This structure has made the movable mirror system hard to be miniaturized for the following reasons: 1) Large size. The sealed moving block and guide rail of the air bearing and the rolling elements of the mechanical bearing are complex structures, so there are no doubt that their sizes are large. The moving block in this mini-scale mechanical bearing is over ten millimeters in length and width and several millimeters in thickness. For example, a mini-scale linear mechanical bearing (model: SEBS 5B) from a custom bearing manufacture, Nippon Bearing [20], has the dimension of $12 \mathrm{~mm} \times 16.9 \mathrm{~mm} \times 4.5 \mathrm{~mm}$. By adding the guide rail and motor, the 
overall length of the movable mirror is several tens millimeters. The dimension of the mini-scale air bearing is even larger than the mechanical bearing [21]. 2) High cost. Air bearings are usually more expensive than the mechanical bearing. And mechanical bearing requires lubricant to be injected to the moving block through a special springe, so additional cost is required. 3) Sensitive to work environment. Both types of bearings are complex instruments, so they are sensitive to environment, such as temptation, humidity and shock.

\subsection{Micromirror}

Micromirrors have been extensively developed, which can generate rotation or translation to be used in display [22]-[33], optical switch [34]-[37], adaptive optics [38][44] and FT spectrometers [45]-[54]. In recent years, the translation micromirror based FT spectrometer has been widely studied and put into production. MEMS based micromirrors have multiple advantages: 1) space saving because of the high integration; 2) low cost due to the batch production; and 3) ability to work under various environment, such as vibration. Because the mass of the moving part in MEMS based micromirror is negligible, the impact on the micromirror is relatively small under a large acceleration. There are three requirements on the micromirror to be applicable in the miniaturized FT spectrometer: 1) large displacement, 2) high surface quality, and 3) linear motion.

\subsubsection{Micromirror actuators}

For applications of FT spectrometer, the important performance specifications include the quasi-static translation range and the response speed. Larger translation leads to higher resolution of the FT spectrometer and higher response speed renders more sampling points within the same measurement interval (subsequently more accurate) and fast measurement. The translation needs to be in the quasi-static mode because FT spectrometer requires the movable mirror to move at constant velocity or in a rapid step motion with equal displacement at each step. Otherwise, 'spectral artifacts' [55][56] will be produced. The variable velocities will lead to the mixture of the original wavelength, emitted from the source, with the wavelengths generated by the movable mirror due to the variable velocities. 
Currently, there are mainly three types of MEMS based actuators. Electrostatic, electromagnetic and electro thermal actuator based micromirrors have been developed as the translation mirror in the Michelson interferometer for FT spectrometer.

1) Electrostatic actuator

This type of actuator based micromirror is the most popular [57]-[63]. Advantages of electrostatic actuators include: a) Simple structure. For example, a parallel plate actuator can be constructed by two parallel conductive surfaces. b) High dynamic response speed. The transition speed depends on the charging or discharging time constant, which is a very small for the good conductors. The disadvantages of electrostatic actuators include:

a) High working voltage. Several hundred volts are required to achieve several tens microns transmission for linear motion micromirror, and over a hundred volts are required to rotate the rotation mirror a few degrees [64]. b) Short displacement in quasistatic model. For a pair of parallel plates, one of which is fixed and the other one is movable, an electrostatic force $\mathrm{F}$ will be generated when a voltage is applied on the plates and its magnitude can be calculated as below:

$$
F=\frac{1}{2} \frac{\varepsilon A V^{2}}{d^{2}}
$$

where $\varepsilon$ is a dielectric constant, $\mathrm{A}$ is the area of each plate, $\mathrm{V}$ is voltage and $\mathrm{d}$ is the distance between two parallel plates. It is clearly seen from equation 1.1 that the magnitude of $\mathrm{F}$ reduces significantly with the increase of $\mathrm{d}$ when $\mathrm{V}$ is same. Therefore, the electrostatic force $\mathrm{F}$ is considered as a short-range force which is most effective when $\mathrm{d}$ is only several microns [65]. Currently, most micromirrors can achieve quasi-static translation of a few tens of micrometers. For example, electrostatic in-plane comb-drive micromirror can achieve a translation of $25 \mu \mathrm{m}$ to $38.5 \mu \mathrm{m}$ with corresponding spectral resolution of $200 \mathrm{~cm}^{-1}$ and $130 \mathrm{~cm}^{-1}$ [66][67], and the electromagnetic actuators driven micromirrors can achieve a displacement of $54 \mu \mathrm{m}$ with the corresponding spectral resolution around $100 \mathrm{~cm}^{-1}$ [17]. To achieve large displacement, most electrostatic actuators need to work under resonant frequencies. For example, as shown in Figure 1.11, it is reported that the micromirrors working at resonant mode can achieve several hundred or even over $1000 \mu \mathrm{m}$ translation [45][68][69]. However, due to the non-quasistatic movement, compensation and vacuum package are required and consequently they are not widely used. 


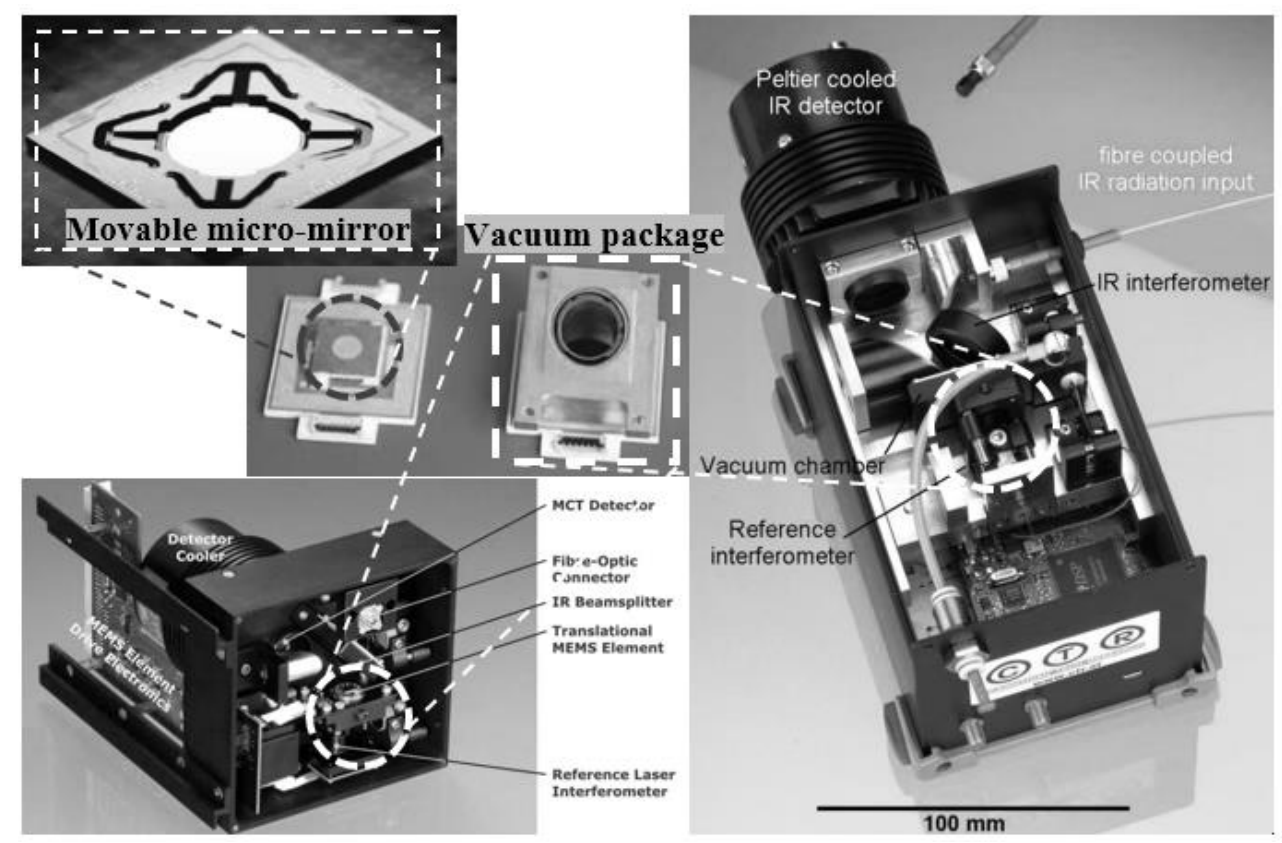

Figure 1.11 Electrostatic actuator working at resonant frequency [68]

2) Thermal actuator

MEMS based thermal actuator, such as [70]-[76], is based on the thermal expansion property of semi-conductor and metal to achieve displacement. For example, the lateral driven thermal actuator utilizes the asymmetry of the structure, as shown in Figure 1.12a, which is composed of two arms made of the same material with different sizes. Since the two arms have different heating powers and thermal expansions, the lateral displacement is achieved after current is applied. A bimetallic actuator is composed of two materials bonded together in vertical direction, which have the same size but different linear CTE. An example of the bimetallic actuator is shown in Figure 1.12b, in which the top layer is made of $\mathrm{SiO}_{2}$ and the bottom layer of $\mathrm{Al}$, whose CTEs are $0.35 \mathrm{ppm} / \mathrm{K}$ and $25 \mathrm{ppm} / \mathrm{K}$, respectively [65]. Therefore, as the temperature increases, Al layer expands more than $\mathrm{SiO}_{2}$ layer, so that the actuator bends upward.

Compared with the electrostatic actuator, the thermal actuator, as the example shown in Figure 1.13, has relatively large displacement, i.e., more than $100 \mu \mathrm{m}$ or even several hundred microns [47][71][77]. However, their response speeds are very slow, e.g. several seconds to tens of seconds for $10 \mu \mathrm{m}$ to several tens micrometer translation, due to the nature of thermal actuation including the heating and cooling cycle. Their settling time is 
in the range of seconds to more than 10 seconds. In addition, high power operation is required because it uses current to generate ohmic heating.

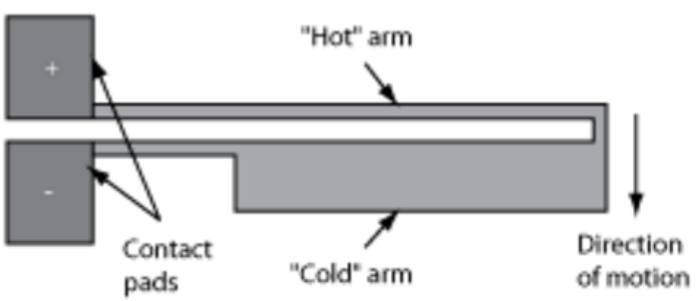

(a)

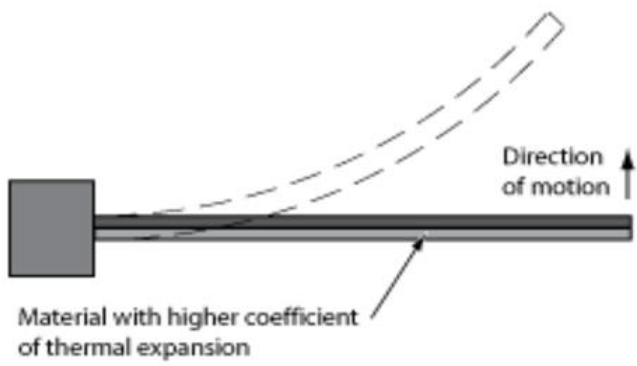

(b)

Figure 1.12 Two types of electro-thermal actuators. (a) Top view of a lateral driven thermal actuator; (b) Side view of a bimetallic actuator [65]

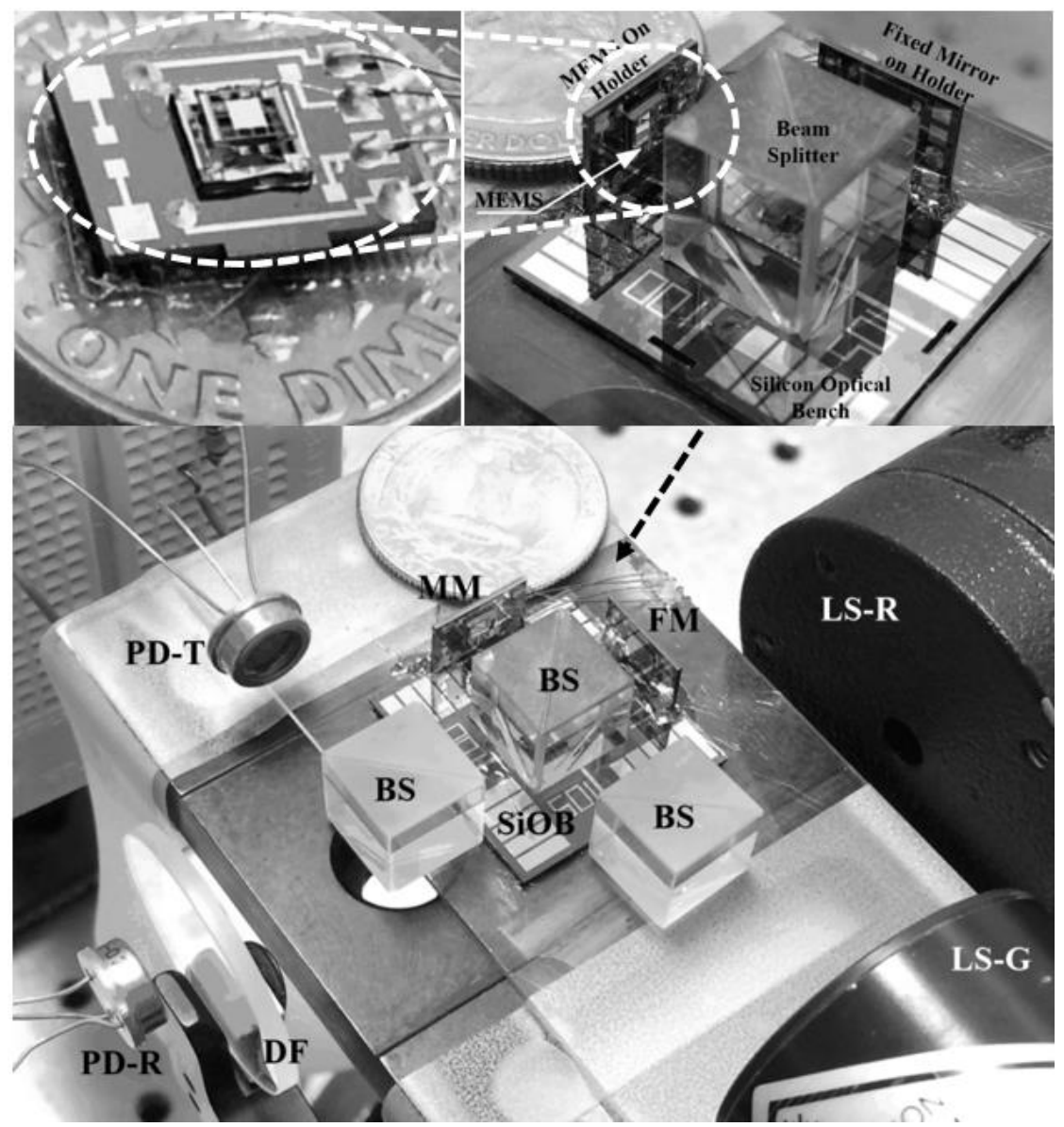

Figure 1.13 Thermal actuator based FT spectrometer [47] 


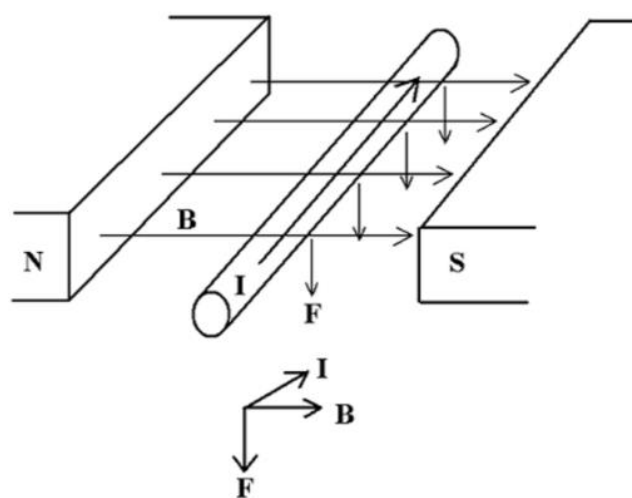

(a)

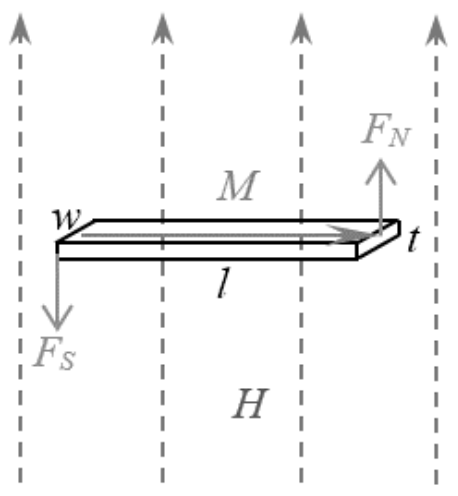

(b)

Figure 1.14 Lorontz force and Magnetostatic force. (a) Wire forced by Lorontz force after current appiled; (b) A piece of iron forced in the magnetic field

\section{3) Magnetic actuator}

The magnetic actuator takes advantage of the magnetic field to generate force or torque to drive the micromirror. Lorentz force [78]-[89] and magnetostatic force [90]-[114] based magnetic actuators are most common. Lorentz force actuator is based on the interaction between the current carrying conductor and the external magnetic field, as shown in Figure 1.14a. For a moving charge $q$, when it enters a magnetic field $B$ with velocity $v$, it is subjected to Lorentz force [65]:

$$
\vec{F}=\overrightarrow{q v} \times \vec{B}
$$

and its magnitude is [65]:

$$
F=q v B \sin \theta
$$

where $\theta$ is the angle between $v$ and magnetic field $\left(\theta<180^{\circ}\right)$. The magnitude of Lorentz force on a wire with unit length $l$ is [65]:

$$
F=I l B \sin \theta
$$

where $I$ is the current through the wire and $B$ is the magnetic field.

Magnetostatic force can be considered as the interacting force between two magnetic poles in the static magnetic field, as shown in Figure 1.14b. When an iron sheet is placed in a uniform magnetic field, its magnetization direction is primarily determined by the shape anisotropy, which leads to the formation of magnetic poles on two ends of the sheet. A concentrated force acting on each monopole can be expressed as [65]:

$$
F=M(w t) H
$$


where $w$ and $t$ are the width and thickness of the cross section of the iron sheet, $M$ is its magnetization vector and $H$ is the strength of magnetic field.

The electro-magnetic actuator has a number of advantages: a) Eliminating electrical wires. The electro-magnetic actuator works under magnetic field instead of driven by voltage or current, therefore no electrical wires is required. b) No threat on human safety. Large electric field or high temperature are potential dangers to the operators since they could cause dielectric breakdown, burn, scald, etc., while large magnetic field has little effect on human. c) Low cost. Using a permanent magnet to provide the external magnetic field is a solution with low cost and no power consumption. d) Relatively large displacement. The micro scale devices can generate considerable force or torque to achieve certain displacement when driven by the electro-magnetic actuator. Certainly, the electro-magnetic actuator also has disadvantages: a) The moving layer of a magnetostatic micromirror has to be made of ferromagnetic material, such as iron, nickel or alloy. b) Relatively large size to achieve enough magnetic field. The chip space limits the number of coil turns so that the magnetic field and the magnetic force are not large enough.

\section{4) Piezoelectric actuator}

The piezoelectric actuator is also wildly used for the translation micromirror. By comparing with others 3 types of actuators, the piezoelectric actuator has the advantages, such as the accurate displacement, large force and high response speed, because its displacement is directly achieved by the solid deformation when the current is applied [115]. However, the large displacement, e.g. more than a few tens micrometer displacement, can only be achieved under the resonant frequency with the deformation of $0.1 \%$ for the classic piezoelectric materials. For example, a $77 \mathrm{~mm}$ long stack piezoelectric actuator can only displace about $70 \mu \mathrm{m}$ under $17 \mathrm{kHz}$ resonant frequency, as the Figure 1.15 shows, which is the maximum displacement achieved by Physik Instrumente, a leading piezo device manufacturer [116]. Because the relative small quasi-static displacement, the piezoelectric actuator working in the quasi-static mode is usually used in the fine adjustment system, such as in the control system for the FabryPerot (F-P) spectrometer [117]-[119], but not suitable for FTIRs which requires large quasi-static displacement. 


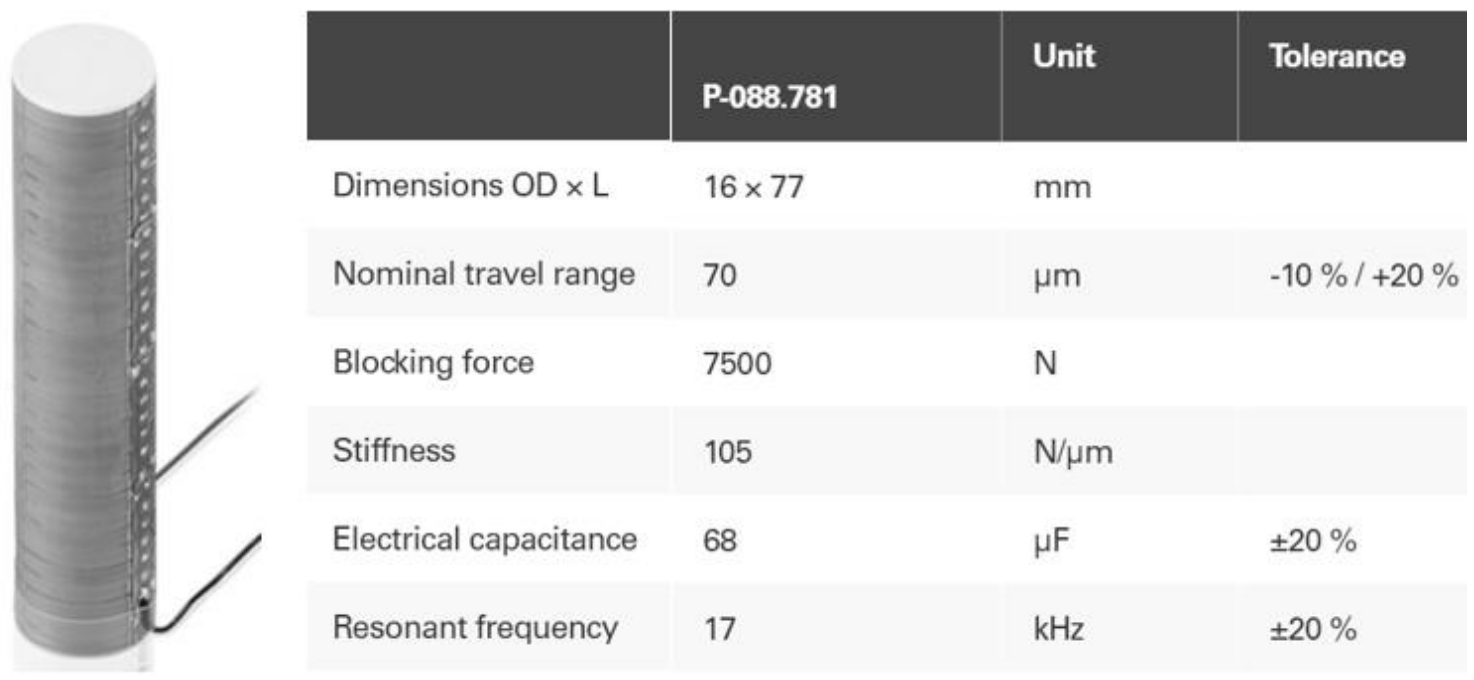

Figure 1.15 Piezoelectric actuator property [116]

\subsubsection{Surface quality of the micromirror}

High surface quality is important when the micromirror works as an optic device in the spectrometer, so its roughness and the radius of curvature are two most critical parameters. The incident intensity of a beam is reduced when it is diffused by a mirror with poor surface quality, which affects the accuracy of the measurement results. Current MEMS technologies, such as LIGA-like [120], SUMMiT-V [121] and bulk micromachining technology [122], can provide surface texture with a roughness of $20 \mathrm{~nm}$ - $40 \mathrm{~nm}$ and a curvature radius of $0.68 \mathrm{~m}-2.65 \mathrm{~m}$ [74][123]. Compared with the macromirrors used in the conventional FT spectrometer which have roughness less than nanometer scale and the curvature radius about tens of meters, it is impossible to achieve by current MEMS technologies. Furthermore, lots of mature commercial MEMS fabrication processes, e.g. MUMPs [124], have the advantages such as low cost and standard processing, which make them suitable for the fabrication of microactuators with large displacement [25][26], but the surface quality is poor because releasing holes on the moving layer are required to pass the solution through for releasing purpose.

\subsubsection{Translation of the micromirror without tilting}

Tilt existing during the motion of the translation mirror affects the quality of the generated interference pattern. As shown in Figure 1.16, the reflected beams from the fixed and the movable mirror cannot combine perfectly if the movable mirror tilts during motion. As the result, the resolution of the generated spectrum degrades. This issue does 
not only exist in the micromirror but also in the conventional movable mirror in Michelson interferometer. To overcome the tilting, many techniques have been developed. For example, (1) using the air bearing or the rolling elements linear mechanical bearing [125], the tilt of the movable mirror can be reduced to $0.008^{\circ}$ in the conventional Michelson interferometer; (2) using the dynamic compensation system [126][127], the position of the movable mirror can be adjusted when various voltages are applied on the multiple actuators; (3) using other optical elements instead of the movable mirror, such as the corner cube reflectors (CCR) and the cat's eye mirror [128]-[134]; and (4) using the mirror-tilt-insensitive system which is realized by a double-sided plane mirror [135]-[137]. These techniques can effectively reduce the tilt and some can be used in miniaturized FTS, e.g., two tiny CCRs are used in a highly integrated miniaturized FTS [17] and a dual reflective surface based large shock electrothermal micromirror is used in a tilt insensitive FTIRs [77]. However, due to the relatively large size of the bearing and the complex structure of the optical elements, these techniques are not suitable for the miniaturized FTIRs. Using the control system based method, open-loop and close-loop control systems are proposed by professor Xie's group in recent years to reduce the size and improve the accuracy of the FTIRs, in which the tilt of micromirror is reduced to $0.004^{\circ}\left( \pm 0.002^{\circ}\right)$ and $0.003^{\circ}\left( \pm 0.0015^{\circ}\right)$ respectively. However, it only works for the micromirror driven by multiple actuators [47][70][77].

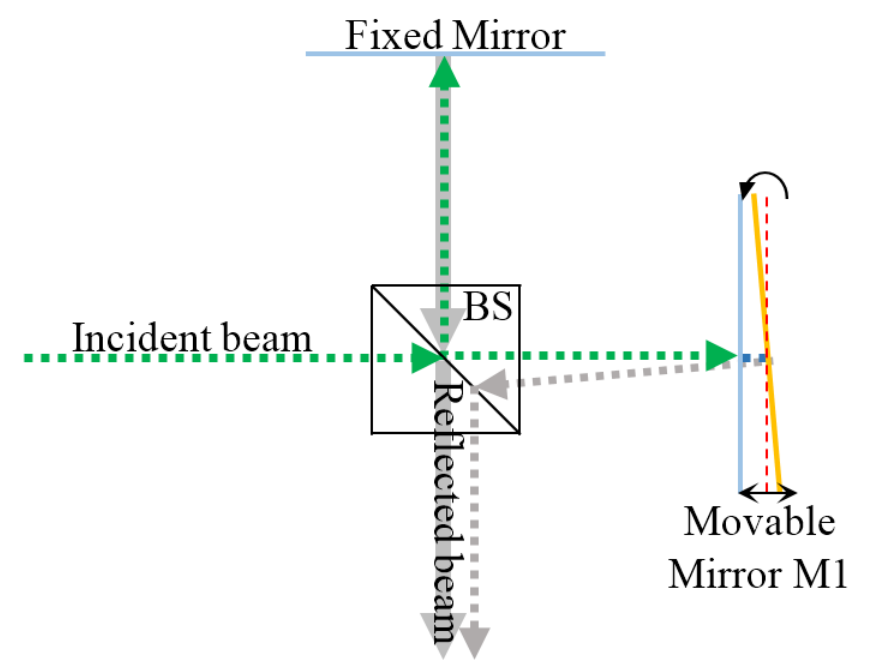

Figure 1.16 Tilt exists in conventional Michelson interferometer 


\subsection{Objectives of the thesis}

\section{Objective 1:}

To develop of a translation micromirror to work as the movable mirror for Michelson interferometer based FT spectrometer. The micromirror will be able to achieve a large quasi-static displacement, e.g., over $100 \mu \mathrm{m}$, and high surface quality, e.g. close to the surface quality of the conventional movable mirror in a Michelson interferometer. The actuator of the micromirror to be developed will be suitable for both simple and mature surface micromachining processes. The simulation, fabrication and prototype testing will be conducted on the novel actuator to be developed.

This micromirror is developed in the following published paper:

- Y. Xue and S. He, "A translation micromirror with large quasi-static displacement and high surface quality," J. Micromechanics Microengineering, Vol. 27, Issue 1, Pages: 1-10, October 2016.

\section{Objective 2:}

To match the surface quality of macromirrors [138] used in the traditional FTIRs, e.g. nanometer scale roughness and over ten meters curvature radius, a bonding technology needs to be developed to bond a high surface quality mirror plate with the moving film of the translation mircromirror. The bonding technology should not be sensitive to the micromachining process, i.e., any process for microactuator fabrication containing the ferromagnetic structure layer can be used and bonded with a high surface quality mirror plate after released. The requirements on this boding technology are: 1) no plastic deformation or damage to the released microactuator; 2) avoid sticking the actuator moving part to the substrate due to the adhesive squeezed out from the bonding surfaces.

This micromirror is developed in the following published paper:

- Y. Xue and S. He, "Magnetic field assisted bonding technology for released micro actuator and mirror surface", J. Microsystem Technologies, DOI: 10.1007/s00542017-3432-7, 2017.

\section{Objective 3:}

An improved micromirror is to be developed by using a novel repulsive force based electromagnetic actuator. The actuator to be developed will have the same advantages as 
the attractive force actuator developed in Objective 1 and overcome its limitations, such as variation in starting position and low repeatability. The simulation, fabrication and prototype testing will also be conducted on the developed improved actuator.

This micromirror is developed in the following paper:

- Y. Xue, H. Zuo and S. He, “A repulsive magnetic force driven translation micromirror," J. Micromechanics Microengineering, in-press.

\section{Objective 4:}

A compensation system is to be designed to correct the tilt of the translation micromirror developed in this thesis, in order to make the translation mirror applicable in the miniaturized Michelson interferometer based FTIRs as a movable mirror. The compensation system will be able to reduce the tilt angle to an allowable range of the FTIRs, so that it can be used in the measurement of substances with wavelength in midinfrared region. In addition, the size of the compensation system should be considered in order to apply it in the miniaturized FTIRs. The system will be tested in a Michelson interferometer and the test results will be analyzed regarding to its compensation capability.

This compensation system is developed in the following paper:

- Y. Xue and S. He, “A tilt compensation system for translation micromirror", Advances in Mechanical Engineering, under review. 


\section{CHAPTER 2 A TRANSLATION MICROMIRROR WITH LARGE QUASI-STATIC DISPLACEMENT AND HIGH SURFACE QUALITY}

This chapter is based on following published paper:

Y. Xue and S. He, "A translation micromirror with large quasi-static displacement and high surface quality," J. Micromechanics Microengineering, Vol. 27, Issue 1, Pages: 110, October 2016.

As per the first objective of the thesis, a large quasi-static displacement with high surface quality translation micromirror is presented in this chapter. The micromirror consists of a magnetic actuator and a mirror plate, which are fabricated separately using two processes and then bonded together. The actuator consists of a moving film which is a $20 \mu \mathrm{m}$ thick nickel film fabricated by MetalMUMPs and a solenoid located underneath the moving film. The moving film is designed to curve up through the residual stress gradient in the nickel film and a curve-up mechanism which includes four trapezoidal plates and anchoring springs. The mirror plate is simply diced from a polished silicon wafer with a metal thin film deposited. The mirror plate is bonded onto the central part of the moving film. A solenoid attracts the moving film and the mirror plate downward to realize translation. A quasi-static displacement of $123 \mu \mathrm{m}$ is achieved at a driving current of $400 \mathrm{~mA}$, with the settling time of $16 \mathrm{~ms}$ downward and $50 \mathrm{~ms}$ upward. A high mirror surface quality is realized, e.g. $15.6 \mathrm{~m}$ of curvature radius and $2 \mathrm{~nm}$ surface roughness.

\subsection{Principle of the micromirror}

\subsubsection{Micromirror structure}

The micromirror consists of a mirror plate and an electromagnetic actuator as shown in Figure 2.1. The actuator and the mirror plate are fabricated separately and bonded together afterward. The mirror plate is fabricated by dicing a polished silicon wafer, which is coated with $100 \mathrm{~nm}$ aluminum. This process is simple and low cost and can achieve a high flatness and good surface roughness. The thickness of the mirror plate is $370 \mu \mathrm{m}$. The aperture of the mirror plate is $2 \mathrm{~mm} \times 2 \mathrm{~mm}$. The actuator is fabricated 
using a mature commercially available process, i.e., MetalMUMPs [139]. An electromagnetic actuator is designed with this process to achieve a large translation. The actuator includes a moving film and a fixed part. The moving film includes four trapezoidal plates and a central ring structure for bonding the mirror plate. As shown in Figure 2.1b, the fixed part includes a solenoid and the substrate. In the moving film, four trapezoidal plates are connected to a central ring structure through connection springs and anchored through anchoring springs; a $25 \mu \mathrm{m}$ trench is etched in the substrate. The moving film is made of $20 \mu \mathrm{m}$ thick nickel by MetalMUMPs.

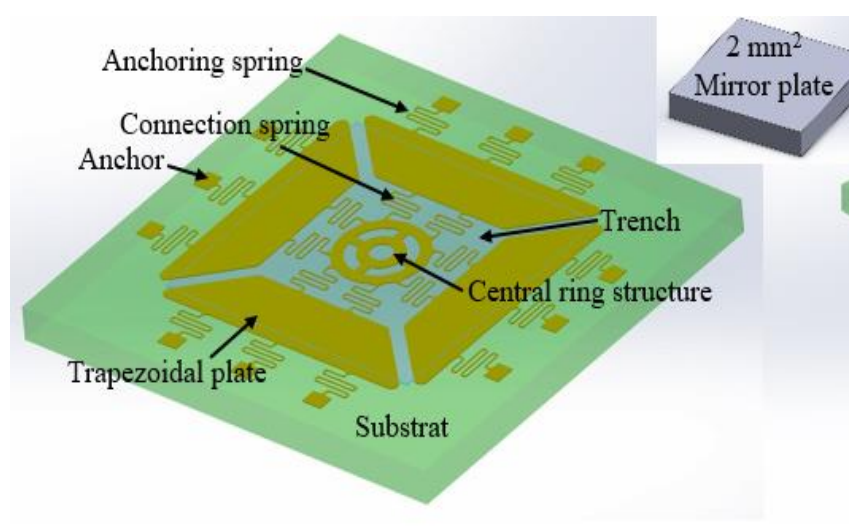

(a)

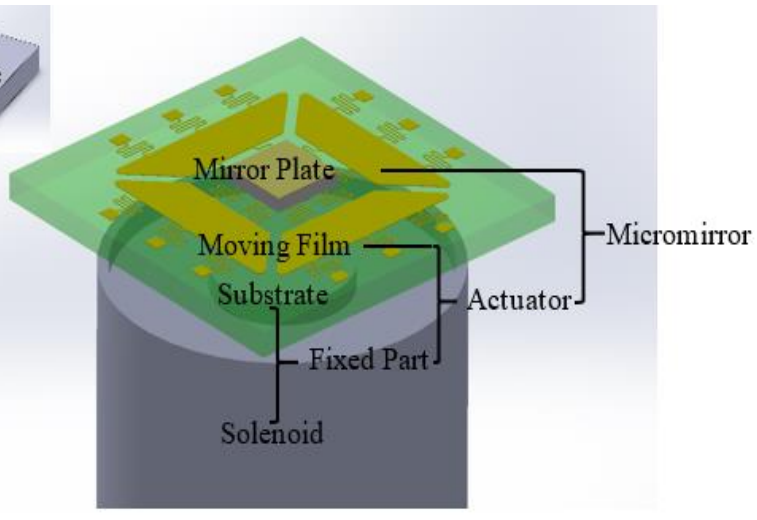

(b)

Figure 2.1 Concept of the micromirror. (a) Actuator moving film plus substrate; (b)

Actuator and the mirror plate

\subsubsection{Raise the central ring structure}

A curve-up mechanism is designed to raise the central ring after release through the negative residual stress gradient in the nickel film of the MetalMUMPS. The curve-up mechanism includes four trapezoidal plates, anchoring springs and the substrate, as shown in Figure 2.2. The central ring structure is raised through the following: (1) Four trapezoidal plates support the central ring structure, with the lower edges anchored through anchoring springs. The whole structure of the moving film curves up due to the negative residual stress gradient in the nickel film, with the central ring structure being the highest point and the lower corners of the four trapezoidal plates being the lowest points; (2) each trapezoidal plate becomes a convex shape with two lower corners warped to move towards the plate center and the substrate. Hence the two lower corners touch the substrate surface and their warping movement is stopped by the substrate. Consequently, 
the remaining part of the trapezoidal plate, along with the central ring structure, curves up.

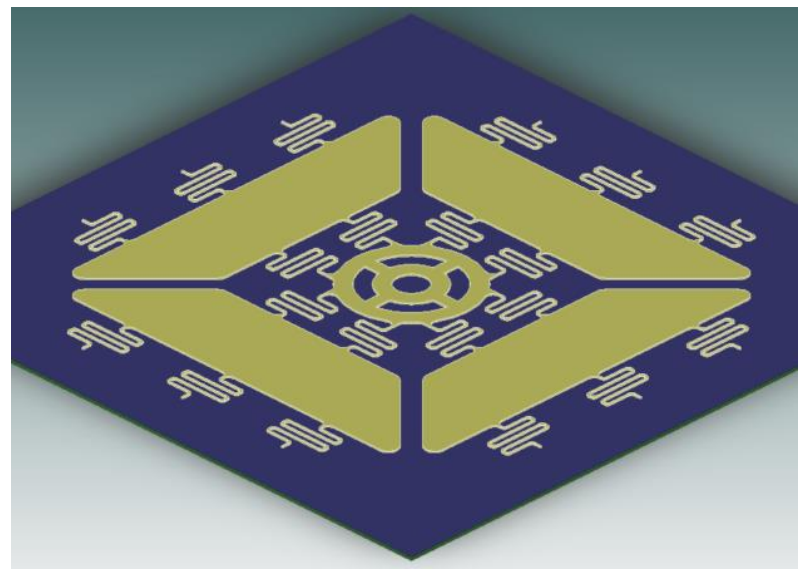

(a)

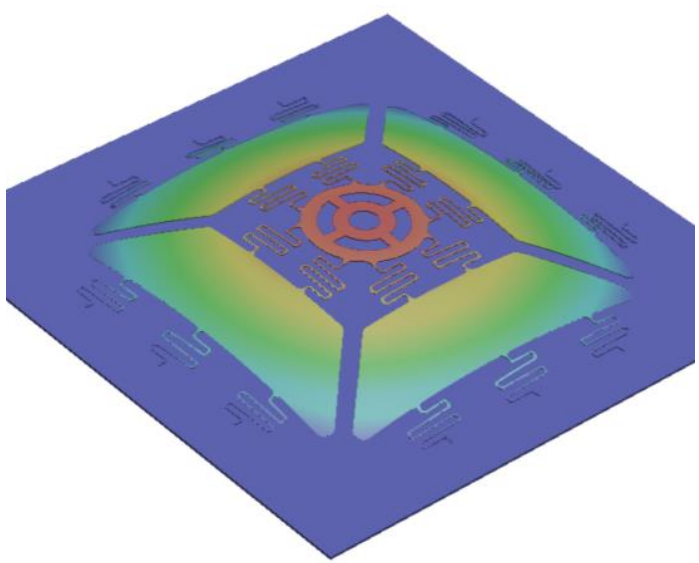

(b)

Figure 2.2 Mirrormirror with no mirror plate bonded curves up through the curve-up mechanism and negative residual stress gradient in the nickel film. (a) Micromirror before release; (b) Micromirror after release

\subsubsection{Driving principle}

The moving film is attracted downward by the magnetic field generated by the solenoid as shown in Figure 2.3a. Because of the large length to thickness ratio of the moving film, the shape anisotropy plays an important role in determining the direction of the magnetization in the trapezoidal plate, which is in-plane [140]. The magnetic field generated by the solenoid is shown in Figure 2.3b, which shows an example of magnetic field of the south pole on the top and north pole at the bottom of solenoid. Based on the anisotropy theory [141][142], an in-plane magnetization is formed in the trapezoidal nickel film with the directions of two forces i.e., FS and FN generated at the top and bottom, as shown in Figure 2.3a, which rotate each trapezoidal plate downward about the anchoring spring. Under the magnetic field, not only the trapezoidal plate but also the central ring structure is magnetized. The magnetization is from the outer face of the central ring to the inner face through the connection beams as shown in Figure 2.3c. The total magnetic force generated by the ring structure is zero. The deformation of the ring structure can be neglected because the connection parts between the inner and outer rings have high stiffness and the bonded mirror plate also increases the rigidity of the structure. 
By controlling the current to the solenoid, the generated magnetic field - and thus the inplate magnetization level and the corresponding force (FS, FN) in each trapezoidal plate can be controlled. Hence the rotation of each trapezoidal plate and the subsequent translation of the central ring structure along with the mirror plate can be controlled, i.e., the higher the current to the solenoid, the larger downward translation of the mirror plate.

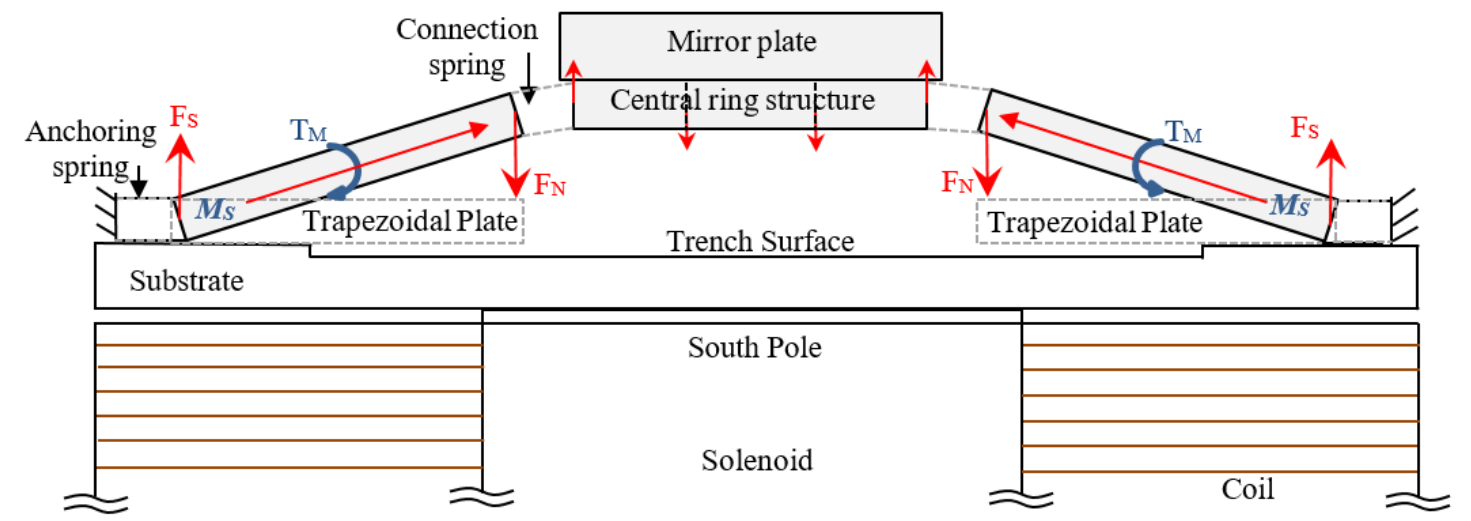

(a)

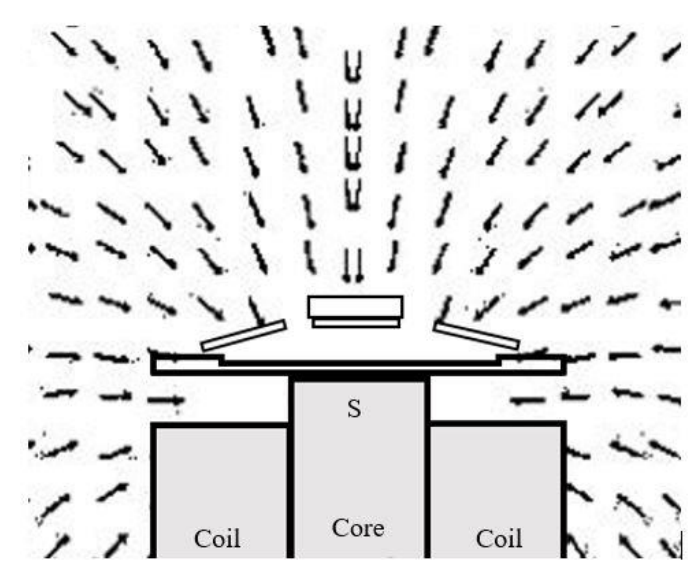

(b)

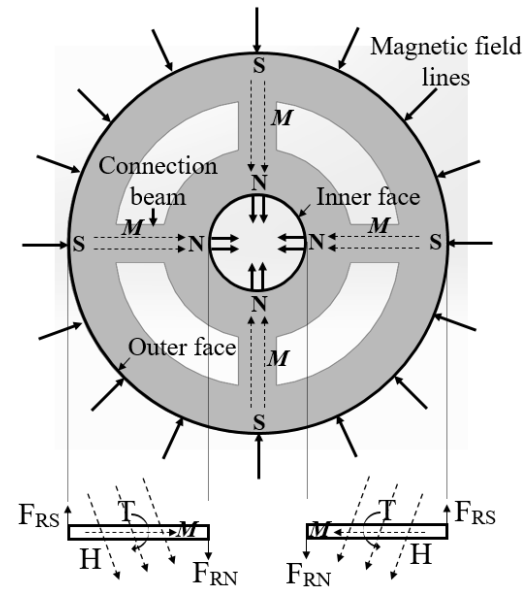

(c)

Figure 2.3 Micromirror principle. (a) Moving film in the magnetic field generated by the solenoid; (b) Magnetic field generated by the solenoid; (c) Central ring structure under the magnetic field

\subsection{FEM simulation}

Using the theoretical model to estimate the approximate value of the micromirror displacement is not applicable for this design because some factors cannot be counted, such as: 1) the moving film is curved up due to the residual stress, 2) the 20 springs 
connecting the moving film with other parts have shape deformation, 3) release holes exist on the moving film as required by the manufacture process, 4) the metal shell of the solenoid has impact on the magnetic field, etc. Therefore, a FEM analysis method is used to find more accurate displacement of the micromirror under different currents.

\subsubsection{Curve up of the moving film}

The curve-up of the moving film due to the residual stress gradient and the curve-up mechanism is simulated in the CoventorWare ${ }^{\circledR}$ [143] simulation environment. The dimensions of the micromirror are shown in Table 2.1. An elastic modulus of $159 \mathrm{GPa}$ and an average residual stress gradient of $-4.72 \mathrm{MPa} / \mu \mathrm{m}[144]$ are used. After release, the lower corners of each trapezoidal plate moves toward the substrate by $1.1 \mu \mathrm{m}$, which is the thickness of the sacrificial layer (Oxide 2 in MetalMUMPs) till touching the substrate surface. The force $\left(\mathrm{F}_{\mathrm{sub}}\right)$ between the substrate and each lower corner of the trapezoidal plate after release is approximated as follows: 1) Simulate the deformation of the moving film due to residual stress gradient without the substrate by adding an upward force $\mathrm{F}_{\text {sub }}$ to each lower corner of the trapezoidal plates; 2) Find the force $\mathrm{F}_{\text {sub }}$ through a few iterations till the lower corners of each trapezoidal plate are at the substrate surface height, i.e., $1.1 \mu \mathrm{m}$ below the moving film's unreleased position. The total force of 1.525 $\mathrm{mN}$ is obtained. The simulated deformation is shown in Figure 2.2b. The central ring structure is raised by $168 \mu \mathrm{m}$ from its unreleased position. A trench of $25 \mu \mathrm{m}$ is opened underneath the central ring structure in the MetalMUMPs process.

\subsubsection{Translation of the mirror plate}

An iteration method is used to simulate the displacement of the moving film under the

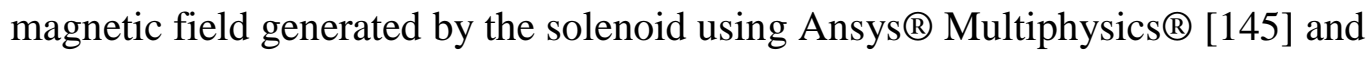
CoventorWare®. The Magneto-Static simulation module of Ansys ${ }^{\circledR}$ Multiphysics software is used to simulate the magnetic force acting on each element of the moving film in the magnetic field. The force obtained from the Magneto-Static module is imported into the CoventorWare ${ }^{\circ}$ MemMech module to simulate the moving film deformation, as described in Appendix A. The deformation is caused not only by the magnetic force, but also the residual stress gradient and the weight of the mirror plate. As described in the residual stress gradient simulation, the upward force $F_{\text {sub }}$ is found after 
iteration till the lower corners of each trapezoidal plate are at the substrate surface height. Due to the symmetric structure, only a quarter of the moving film and a quarter of the solenoid are used in the simulation and the following variables are used, as shown in Figure 2.4.

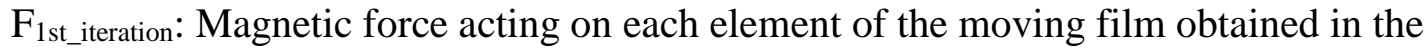
1st iteration of simulation in Magneto-Static module with the moving film shape of the original deformation, i.e., Def_original (defined below).

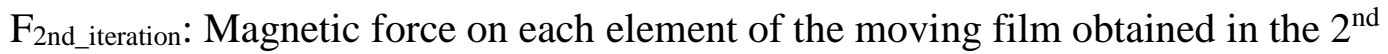
iteration of simulation in Magneto-Static module with the moving film shape obtained in the $1^{\text {st }}$ iteration simulation in CoventorWare ${ }^{\circledR}$ MemMech module, i.e., Def 1st_iteration $_{1}$ (defined below).

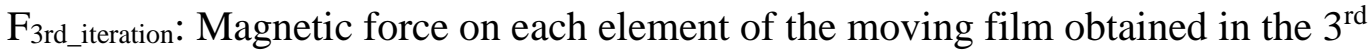
iteration of simulation in Magneto-Static module with the moving film shape obtained in the $2^{\text {nd }}$ iteration simulation in CoventorWare ${ }^{\circledR}$ MemMech module, i.e., Def $f_{2 n d \_ \text {iteration }}$ (defined below).

Def_original: Deformation of the moving film after release due to residual stress gradient in the absence of any magnetic force.

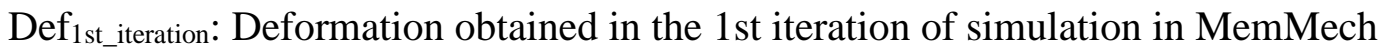
module when applying the residual stress gradient, weight of the mirror plate and the force of $\mathrm{F}^{\text {st }}{ }_{-}$iteration on the moving film.

Def $_{2 \text { nd_iteration: }}$ Deformation obtained in the $2^{\text {nd }}$ iteration of simulation in MemMech module when applying the residual stress gradient, weight of the mirror plate and the force of $\mathrm{F}_{2 \text { nd_iteration }}$ on the moving film.

The iteration of simulation is to obtain the values of variables following the order (as

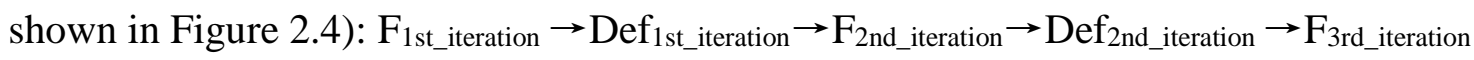
$\rightarrow \operatorname{Def}_{3 \text { rd_itration }} \cdots$ The iteration continues until the deformation is stabilized. In this chapter the deformation is considered as "stabilized" when the variation of deformation is about 3\%. The simulated translations at various currents are shown in Figure 2.10a. The maximum translation is $112.4 \mu \mathrm{m}$ at $400 \mathrm{~mA}$. 


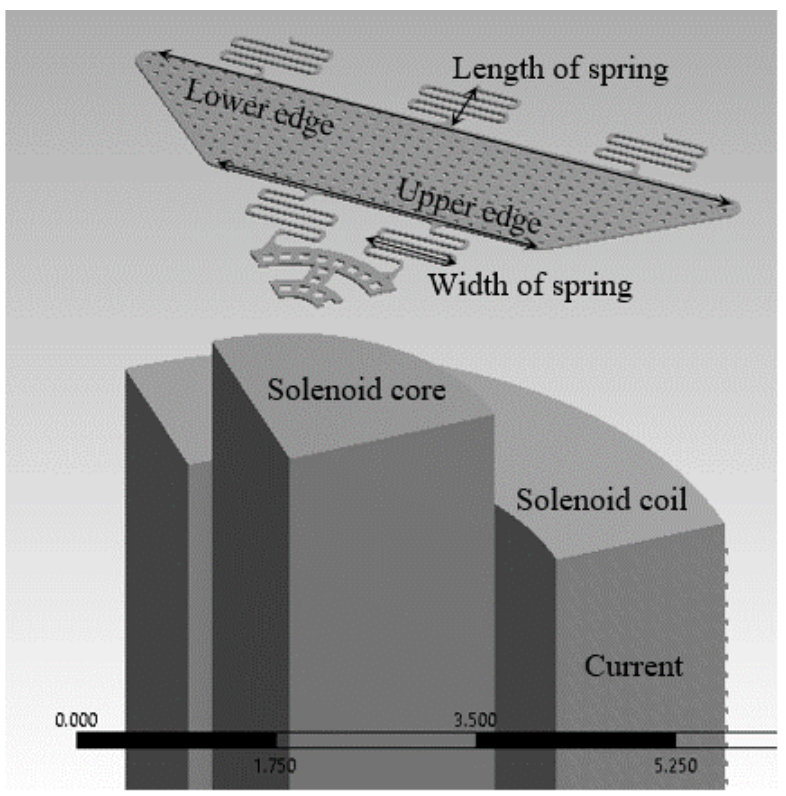

(a)

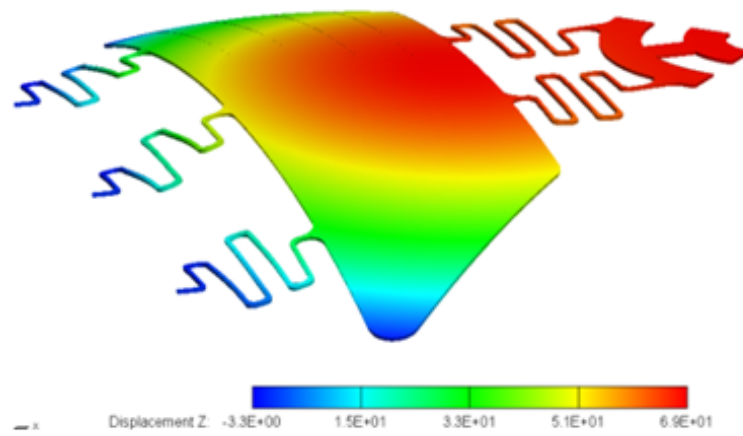

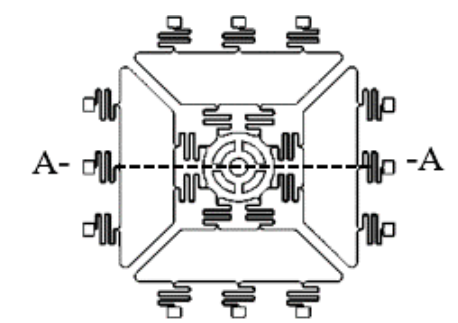

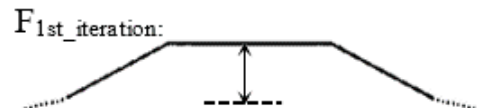

Substrate surface height

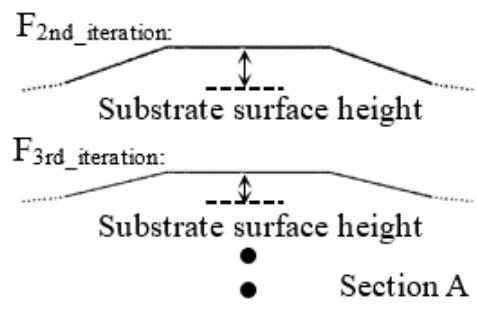

Def-original_Def-force $1^{\text {st }}-1^{\text {st }}$ Iteration:

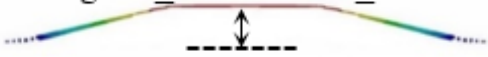

Substrate surface height

Def-original_Def-force $2^{\text {nd }} 2^{\text {nd }}$ Iteration:

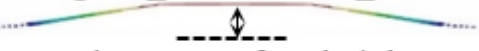

Substrate surface height

Def-original_Def-force $3^{\text {rd }} 3^{\text {rd }}$ Iteration:

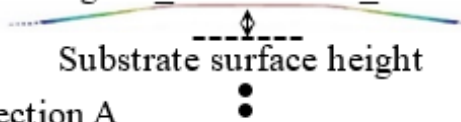

Section A

(b)

Figure 2.4 Combining simulation of magnetic field and mechanical deformation. (a) Magnetic field simulation to obtain the force on the moving film; (b) Simulation of the moving film deformation under the residual stress gradient, mirror plate weight and the magnetic force obtained in (a)

\subsection{Experiments}

\subsubsection{Prototype}

The micromirror is fabricated using the following processes. The moving film is fabricated through MetalMUMPs. The mirror plate is fabricated by slicing $370 \mu \mathrm{m}$ thick polished silicon wafer coated with $100 \mathrm{~nm}$ aluminum on one side. Figure 2.5a - Figure $2.5 \mathrm{c}$ show the moving film and the mirror plate with the dimensions listed in Table 2.1. 
The surface quality of the mirror plate is measured using a Zygo 3D profiler [146], the surface roughness and radius of curvature are $2 \mathrm{~nm}$ and $15.6 \mathrm{~m}$ respectively, as shown in Figure 2.6. The average reflectance of the micromirror is around $95 \%$ as measured with a $635 \mathrm{~nm}$ wavelength red laser, as shown in Figure 2.7.

Table 2.1 Dimensions of the micromirror's moving part

\begin{tabular}{|c|c|c|c|}
\hline Part name & Dimension & Part name & Dimension \\
\hline Mirror plate & $1.9 \mathrm{~mm} \times 1.9 \mathrm{~mm}$ & $\begin{array}{c}\text { Moving film } \\
\text { thickness }\end{array}$ & $20 \mu \mathrm{m}$ \\
\hline $\begin{array}{c}\text { Mirror plate thickness } \\
\text { Upper edge of } \\
\text { trapezoidal plate }\end{array}$ & $370 \mu \mathrm{m}$ & Central ring & $2 \mathrm{~mm}$ (diameter) \\
\hline $\begin{array}{c}\text { Lower edge of } \\
\text { trapezoidal plate }\end{array}$ & $6 \mathrm{~mm}$ & Anchor spring & $0.8 \mathrm{~mm}(\mathrm{~L}) \times 0.9 \mathrm{~mm}(\mathrm{~W})$ \\
\hline $\begin{array}{c}\text { Height of trapezoidal } \\
\text { plate }\end{array}$ & $1.5 \mathrm{~mm}$ & & $0.9 \mathrm{~mm}(\mathrm{~L}) \times 0.8 \mathrm{~mm}(\mathrm{~W})$ \\
\hline
\end{tabular}

A solenoid is designed and integrated with the moving film and mirror plate as shown in Figure 2.5d. The dimensions of the assembled translation micromirror are $15 \mathrm{~mm}$ in diameter and $5.6 \mathrm{~mm}$ in height. The solenoid has parameters listed in Table 2.2 and can attract the moving film and the mirror plate down by more than $120 \mu \mathrm{m}$. However, the price of manufacturing this solenoid is very high due to the low quantity and customized design (no bobbin, small and short core). Instead of using this self-designed solenoid, a commercial solenoid (E-66-38 from Magnetic Sensor System) is used to drive the translation micromirror. Its height is much higher than the self-designed solenoid even both can meet the requirement to drive micromirror with the same amount of translation. An assembled part is shown in Figure 2.5e, which has a $1 \mathrm{~mm}$ deep square shape blind hole opened on top of the part to hold the actuator and a $2 \mathrm{~mm}$ deep circular blind hole drilled on the other side to accommodate the solenoid. The holder is designed to ensure the center of the actuator is on the same line with the central axis of the solenoid. 


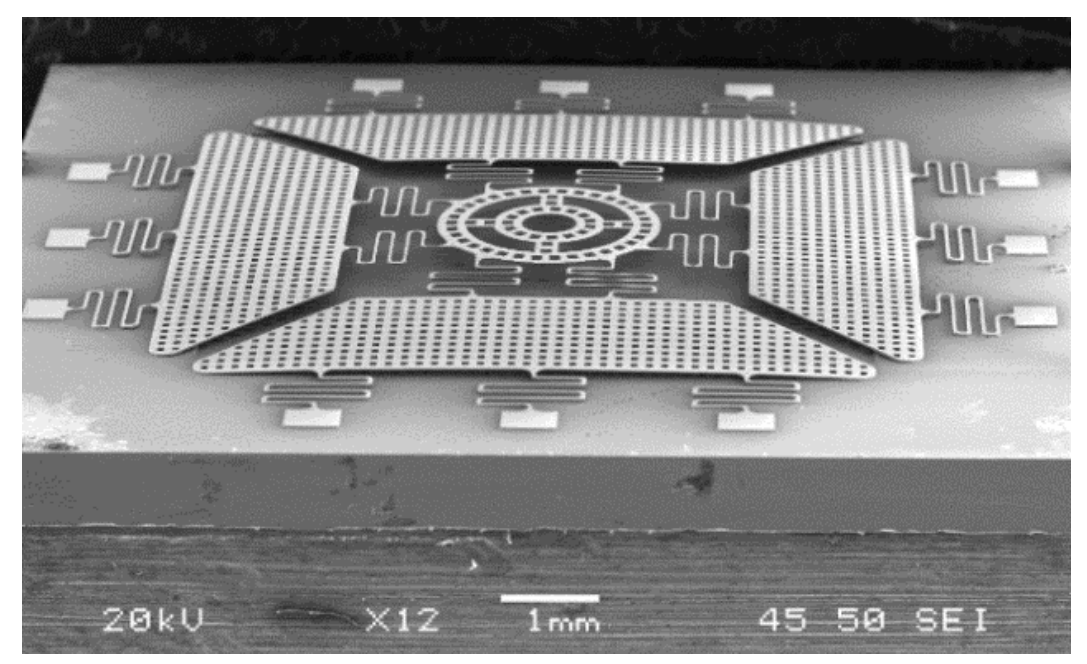

(a)

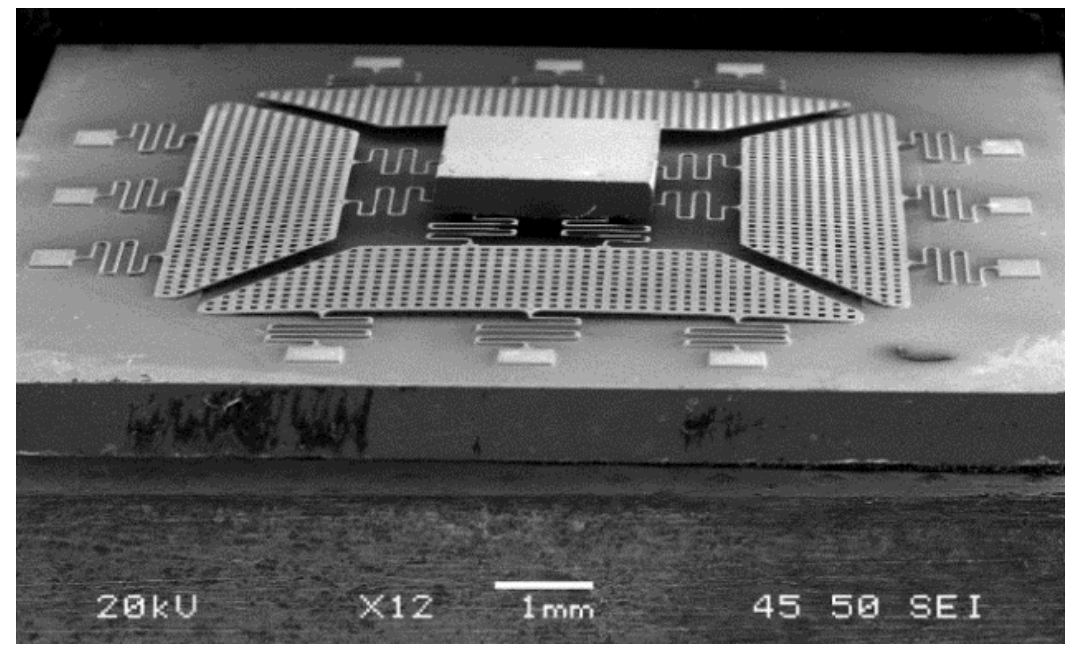

(b)

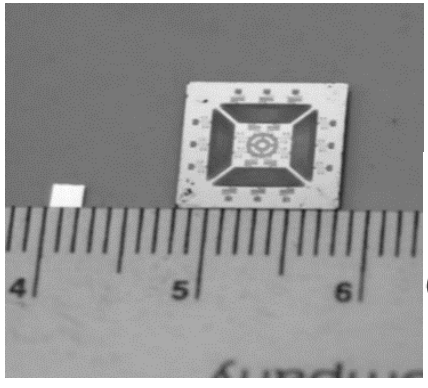

(c)

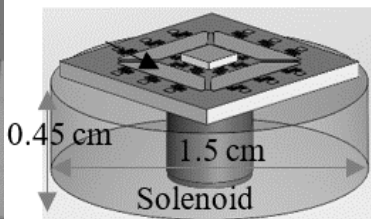

(d)

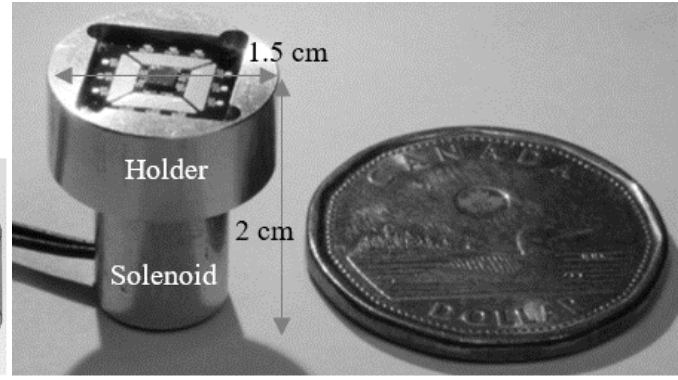

(e)

Figure 2.5 Prototype of the translation micromirror. (a) Moving film (nickel film); (b)

Moving film bonded with the mirror plate; (c) Mirror plate \& moving film chip; (d) Assembled micromirror with self-designed solenoid; (e) Assembled micromirror with commercial solenoid 


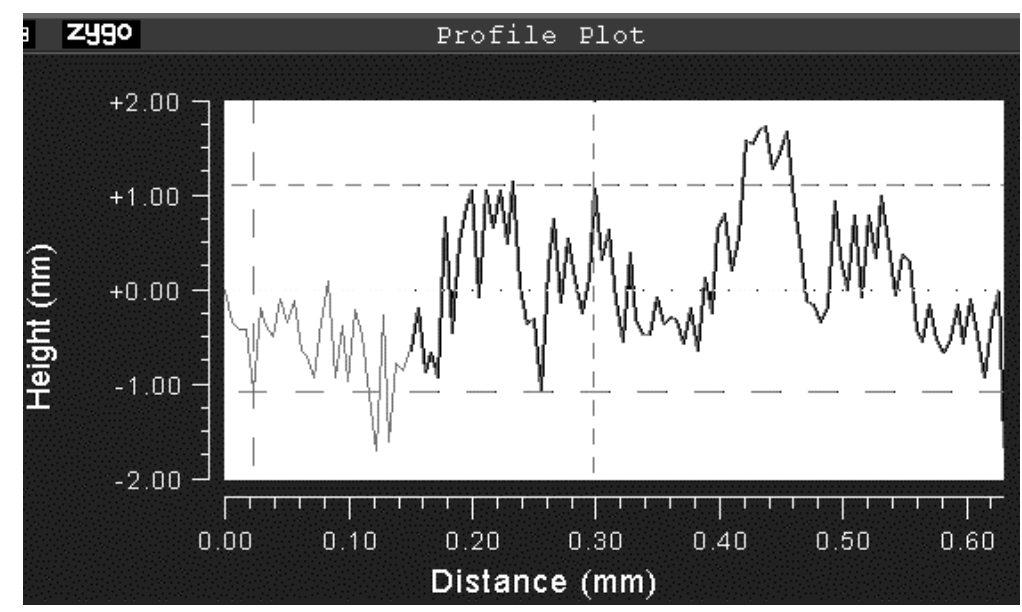

(a)

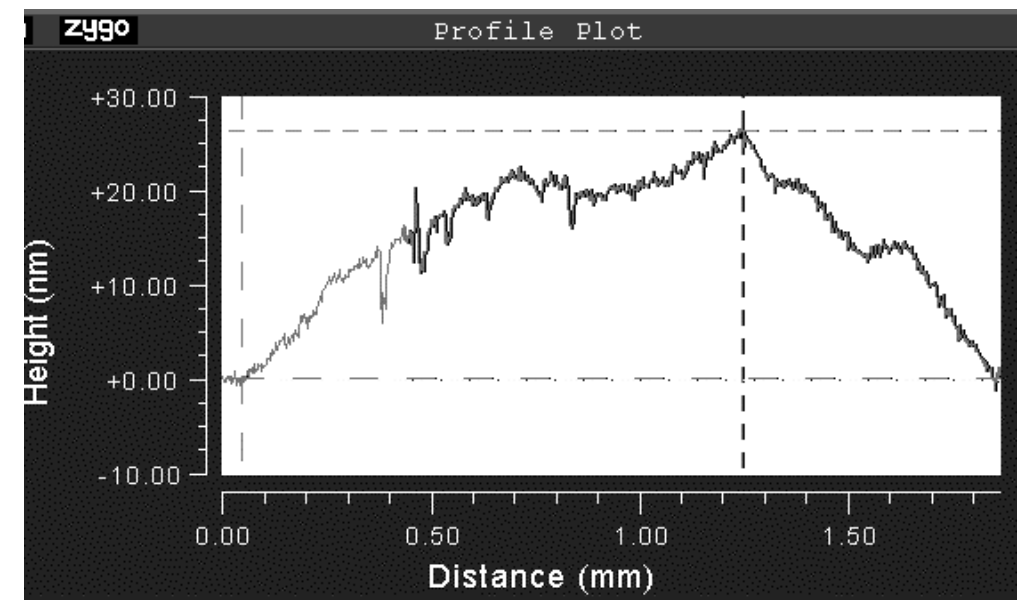

(b)

Figure 2.6 Surface quality of mirror plate. (a) Roughness; (b) Curvature

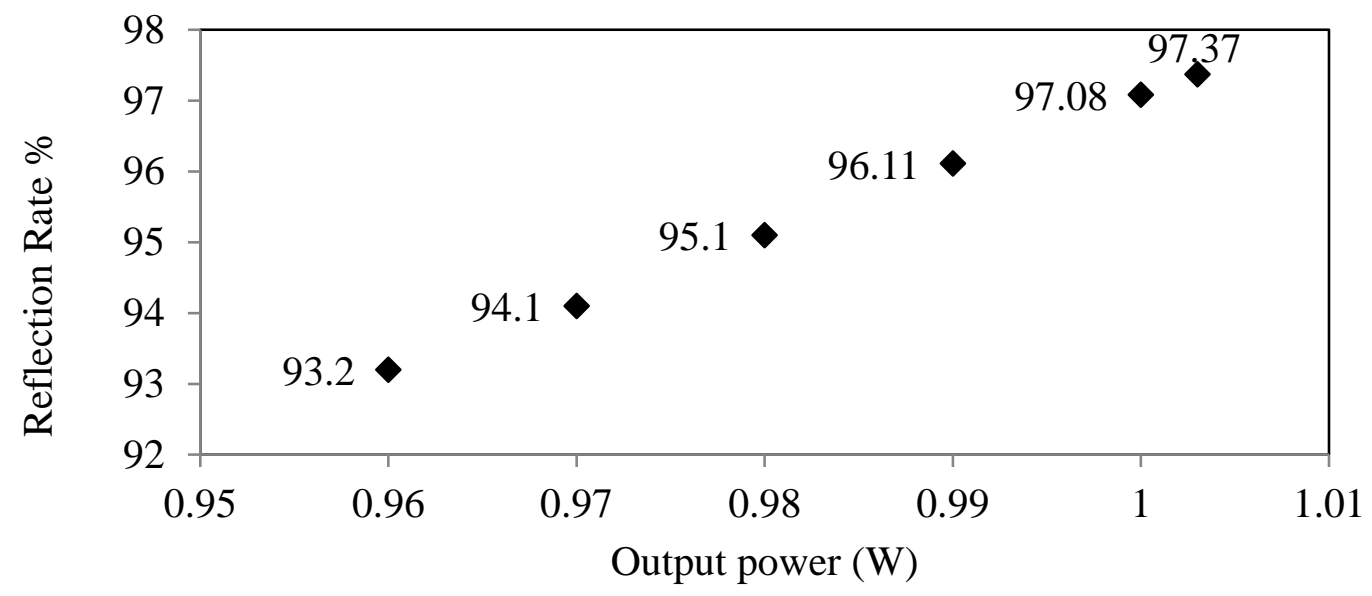

Figure 2.7 The reflectance of the mirror plate (input power $1.03 \mathrm{~W}$ ) 
The bonding process is critical. It is very challenging to bond the released microactuator with the mirror plate without causing any plastic deformation or damage to the released microactuator. A novel bonding technology - "Magnetic field force assisted bonding technology" [147] - has been developed and will be introduced in Chapter 3, which applies non-touching magnetic field force, instead of conventional mechanical force, on the moving part of the released actuator to bring it in contact with the mirror plate. In addition, the bonding pressure is applied through pulling force, instead of compression force as in conventional bonding techniques, so as to avoid any plastic deformation or damage to the released microactuator.

Table 2.2 Dimensions of the custom and commercial solenoid

\begin{tabular}{|c|c|c|c|c|c|c|c|c|c|}
\hline & $\begin{array}{c}\text { Solenoid } \\
\text { Height }\end{array}$ & $\begin{array}{c}\text { Core } \\
\text { diameter }\end{array}$ & $\begin{array}{c}\text { Coil } \\
\text { Height }\end{array}$ & $\begin{array}{c}\text { Coil } \\
\text { diameter }\end{array}$ & MMF & $\begin{array}{c}\text { Magnetic } \\
\text { wire \# }\end{array}$ & Turns & Resistor & Power \\
\hline $\begin{array}{c}\text { Self- } \\
\text { designed } \\
\text { Solenoid }\end{array}$ & $4.5 \mathrm{~mm}$ & $15 \mathrm{~mm}$ & $\begin{array}{l}4.5 \\
\mathrm{~mm}\end{array}$ & $\begin{array}{c}4 \mathrm{~mm} \\
\text { (inner)/ } \\
15 \mathrm{~mm} \\
\text { (outer) }\end{array}$ & $\begin{array}{l}150 \\
\text { AT }\end{array}$ & AWG34 & 730 & $13.56 \Omega$ & $0.6 \mathrm{~W}$ \\
\hline $\begin{array}{c}\text { Commercial } \\
\text { Solenoid }\end{array}$ & $16.8 \mathrm{~mm}$ & $\begin{array}{l}3.85 \\
\mathrm{~mm}\end{array}$ & $\begin{array}{c}10 \\
\mathrm{~mm}\end{array}$ & $\begin{array}{c}5 \mathrm{~mm} \\
\text { (inner)/ } \\
8.5 \mathrm{~mm} \\
\text { (outer) }\end{array}$ & $\begin{array}{l}158 \\
\text { AT }\end{array}$ & AWG34 & 400 & $7.43 \Omega$ & $1.2 \mathrm{~W}$ \\
\hline
\end{tabular}

\subsubsection{The moving film's curve-up}

The moving film of the prototype curves up and subsequently the central ring structure is raised due to the residual stress gradient in the nickel film and the curve-up mechanism, as predicted in the section of 2.1.2. The lower corners of each trapezoid plate touch the substrate surface and the trapezoid plate warps to form a convex shape as shown in Figure 2.8. The average value of the central ring deformation due to the residual stress gradient is around $165 \mu \mathrm{m}$ basing on the measurement results of several prototypes, which is close to the simulation result $(168 \mu \mathrm{m})$ in section 2.2.1. 


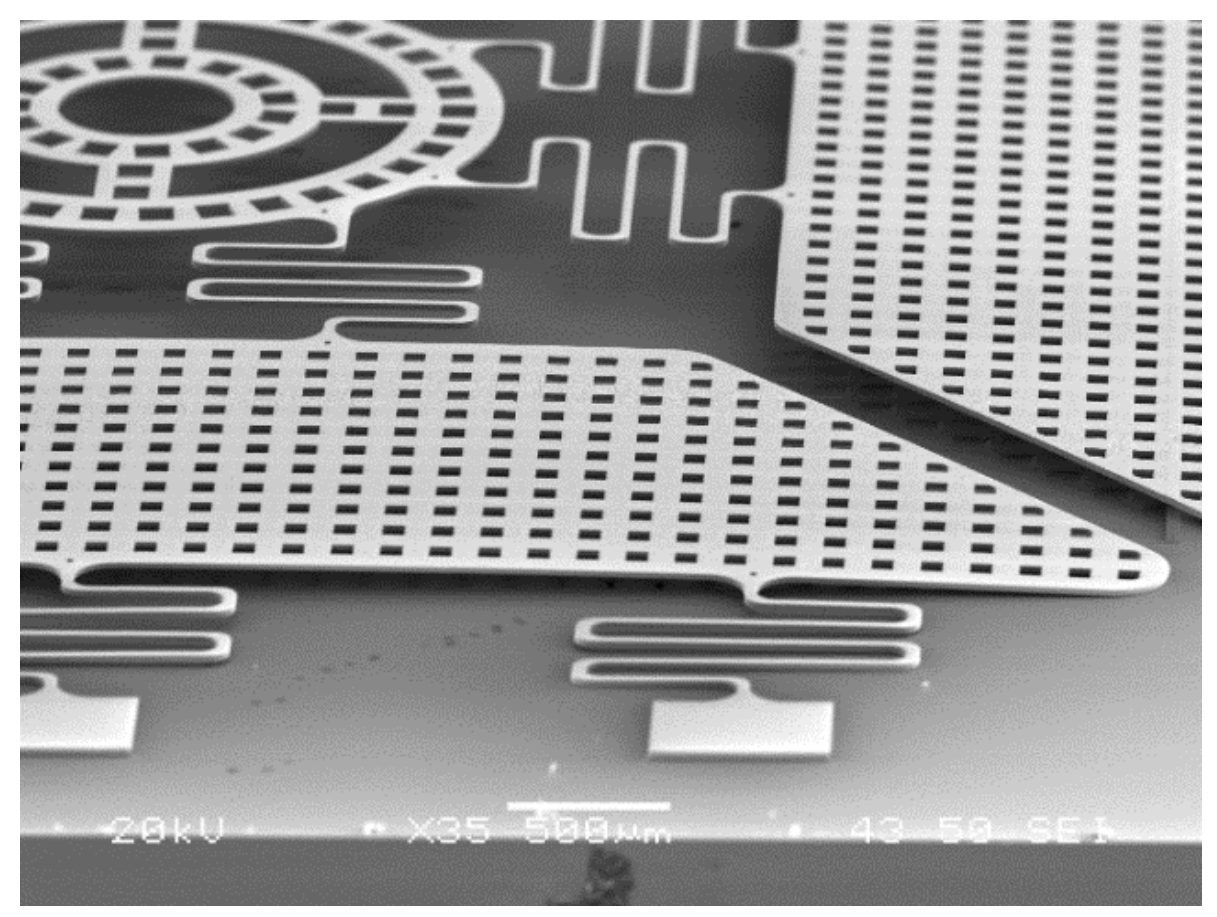

(a)

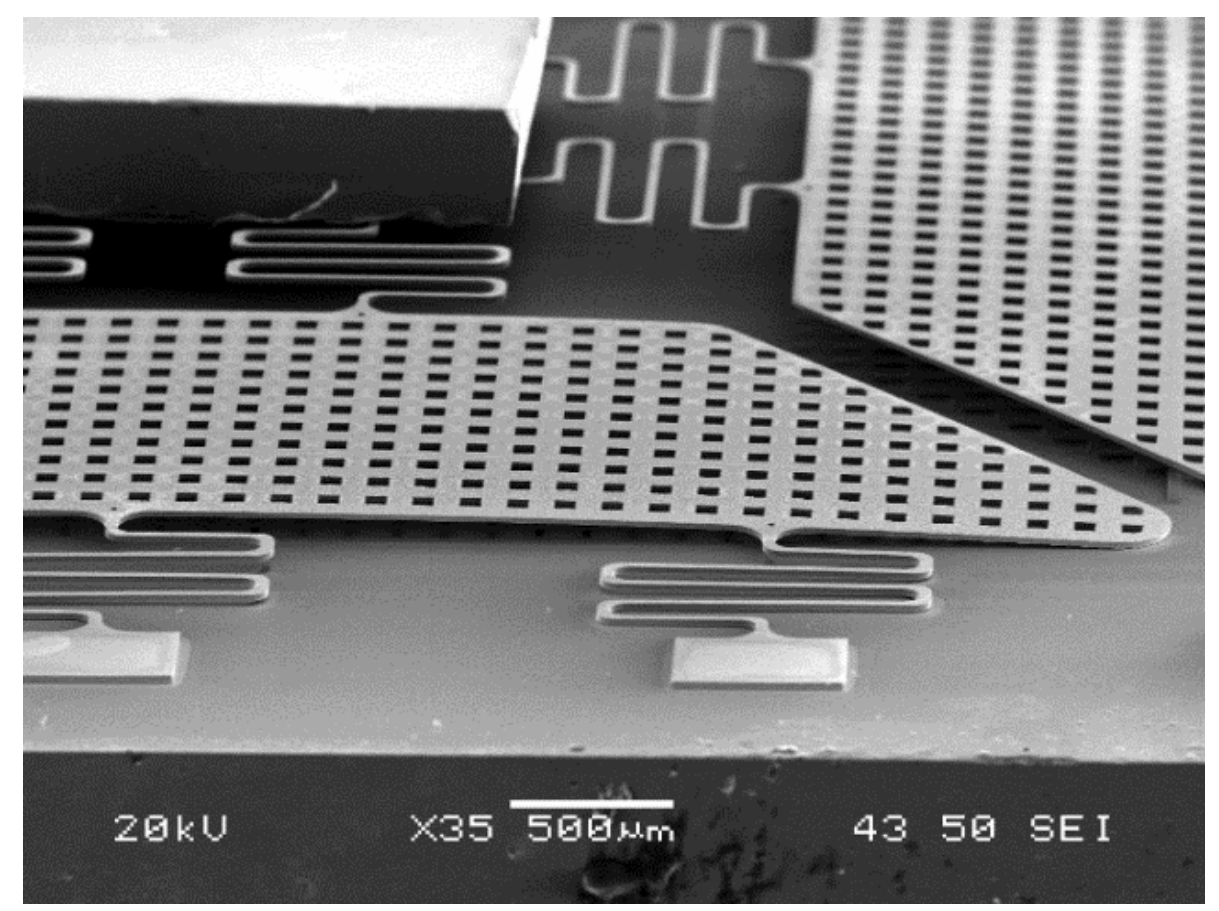

(b)

Figure 2.8 Prototypes of the moving film and the mirror plate. (a) Curve-up mechanism; (b) Curve-up mechanism with mirror plate 


\subsubsection{Quasi-static translation}

The assembled micromirror is measured for its static and dynamic performance using the Zygo 3D profiler and a PSD based dynamic translation measurement setup as shown in Figure 2.9 and Figure 2.11 respectively. In order to protect the micromirror and enable the mounting of it on a stage for dynamic measurement, a mounting holder and a protection cover are added to the micromirror assembly for measurement as shown in Figure 2.9a.

Both the measured and simulated results are shown in Figure 2.10a. The maximum translation is $123 \mu \mathrm{m}$ at $400 \mathrm{~mA}$. The discrepancy between the measurement and simulation at $400 \mathrm{~mA}$ is $7 \%$. Possible reasons for the discrepancy are: (1) The shape of the moving film in the force simulation iteration is manually created based on the movement of the central ring obtained in the deformation simulation iteration. Thus, the shapes of trapezoidal plates and springs in the force simulation are approximations to the real deformation. (2) In the deformation simulation, the force on each individual element of the trapezoidal plate is not directly used. Instead, each trapezoidal plate is divided into five areas along its height and all elements in the same area are treated to have the same magnetic force to simulate the deformation.

The initial tilting of the moving part of the actuator caused by the fabrication process is insignificant, i.e., less than the noise caused by the microactuator's surface. But the tilting angle of the mirror plate is $0.1^{\circ}-0.24^{\circ}$, based on measurement on several prototypes with the trench bottom surface as reference after bonding, even when there is no plastic deformation to the moving structure of the actuator caused by the bonding through experimentally measuring 40 points on the moving structure [147]. However, the height of the micromirror decreases by around $10 \mu \mathrm{m}$ after several movements when current is applied and becomes stable after 10 hours vibration test. A possible reason for the height decrease is because the low corners of the trapezoidal plate are moved laterally in operation. This static tilting of the mirror plate is caused by the non-uniform thickness of the adhesive. However, the static tilting is automatically eliminated when aligning the optics and can be significantly reduced if spinning coating is used to apply the adhesive. 


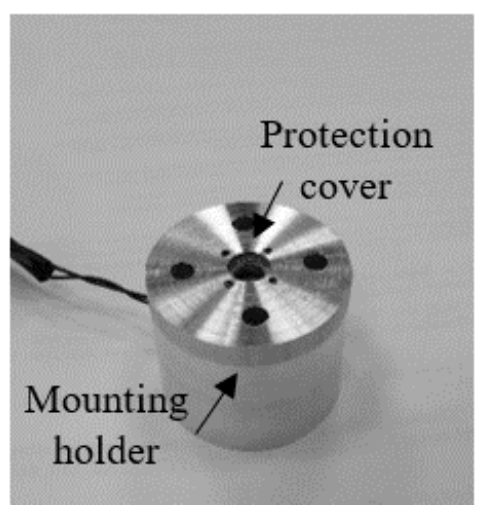

(a)

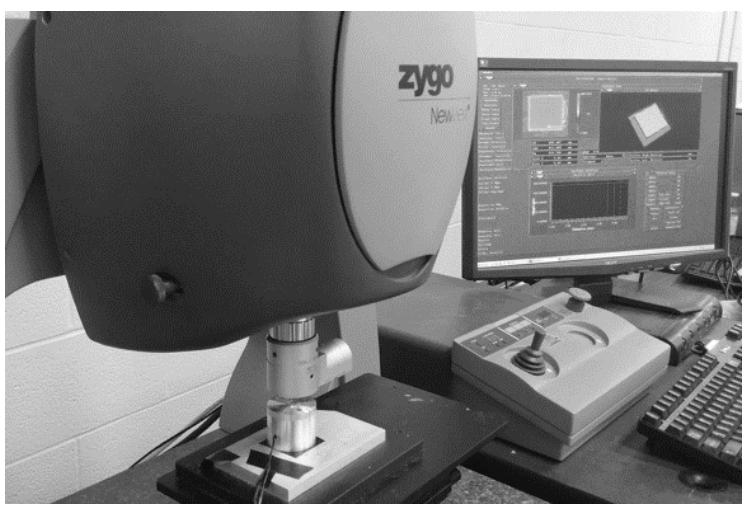

(b)

Figure 2.9 Static translation measurement. (a) Protection cover \& mounting holder; (b)

Static performance measured using Zygo profiler

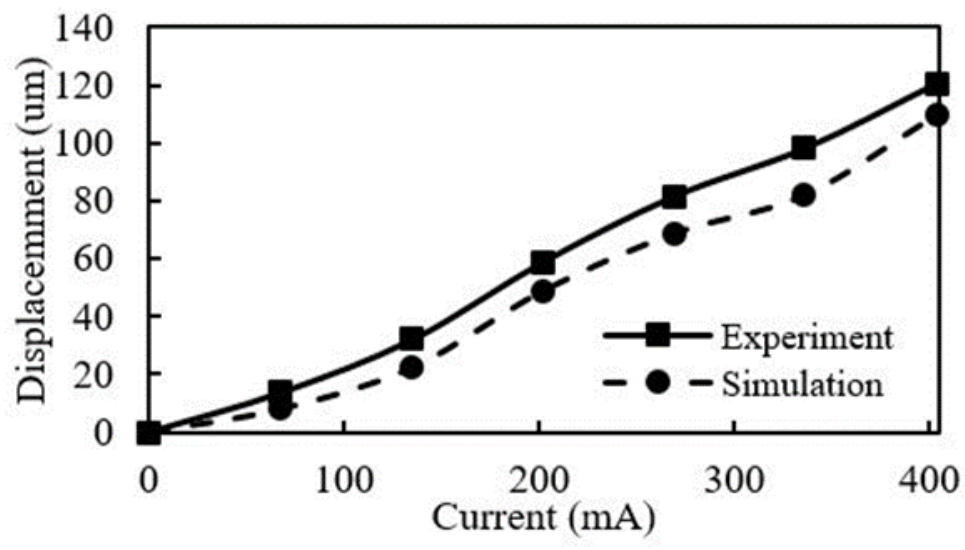

(a)

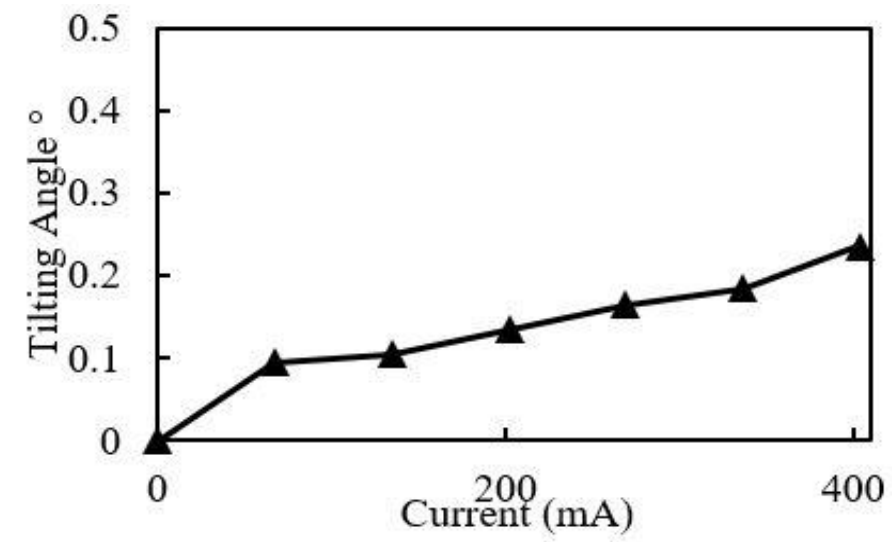

(b)

Figure 2.10 Static performance of the micromirror. (a) Translations of experiment and simulation; (b) Tilting angle of the micromirror 


\subsubsection{Dynamic Response Measurement}

The dynamic performance is measured on a PSD based setup, which includes a laser, a PSD, and rotation stage, as shown in Figure 2.11. A laser beam spots on the mirror plate and is reflected to the PSD surface. When the micromirror translates, the laser spot moves on the PSD surface. The distance of movement of the laser spot on the PSD surface can be measured in the real-time mode and then the translation of the micromirror can be obtained dynamically as shown in Figure 2.12a. Since the micromirror translates with a certain amount of tilting, this also causes movement of the laser spot on PSD. In order to find out the displacement of the laser spot on PSD caused purely by the micromirror's translation, the following method is used. Step 1: apply the current with a square wave and rotate the micromirror by rotating the stage till the maximum displacement along the $\mathrm{y}$-axis on PSD, at which the laser spot movement on PSD caused by tilting constructively superposes with the movement by translation of the micromirror. The tilting axis is parallel to the X-axis of PSD surface as shown in Figure 2.12b; step 2: based on the position the micromirror attained in step 1 , rotate the micromirror by $90^{\circ}$ so that the tilting axis becomes perpendicular to the $\mathrm{X}$-axis of PSD surface, so that the tilting of the micromirror does not cause any laser spot movement along PSD Y-axis. Subsequently, the laser spot displacement along PSD Y-axis is purely caused by the translation of the micromirror.

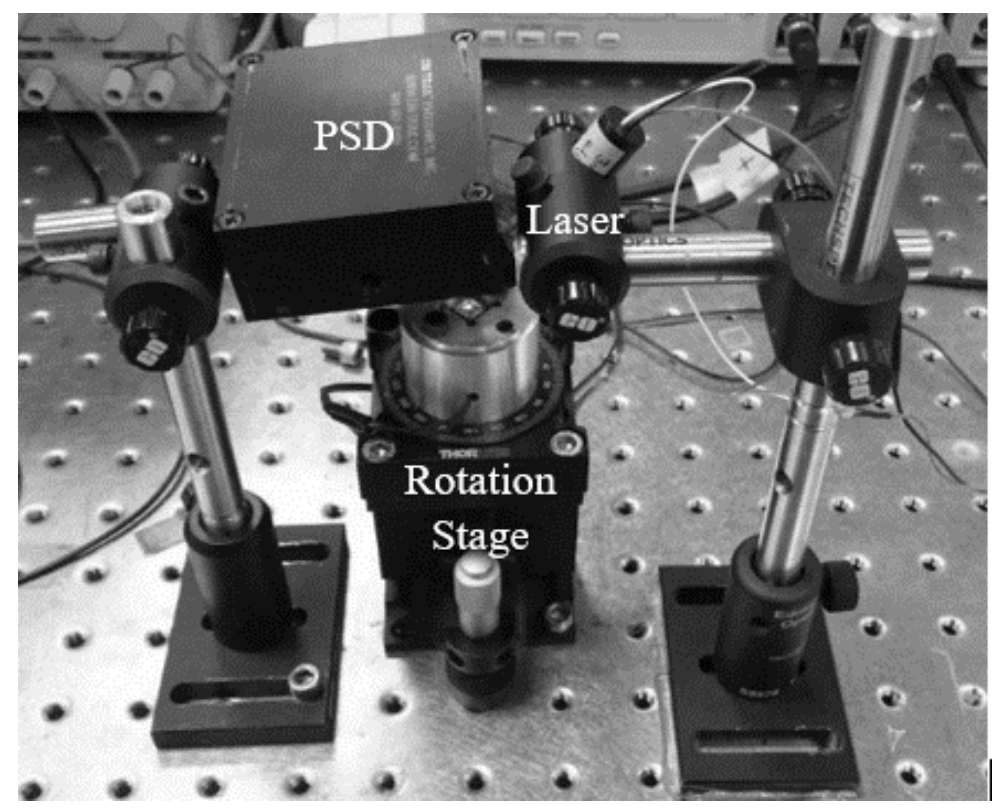

Figure 2.11 Dynamic measurement setup 


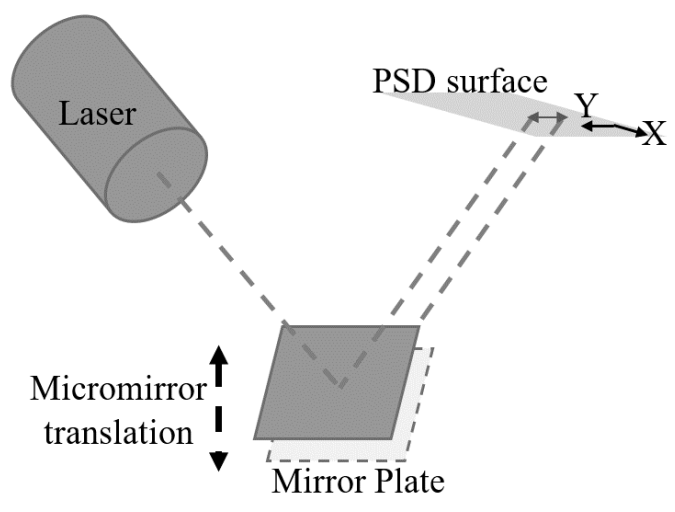

(a)

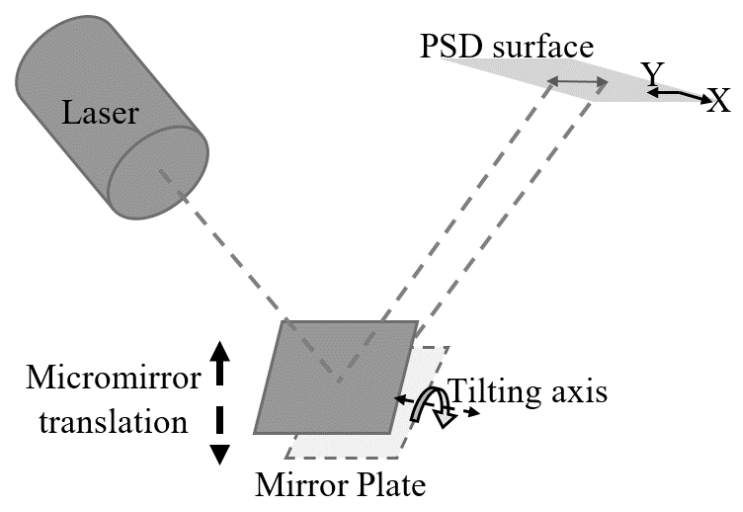

(b)

Figure 2.12 Concept of dynamic translation measurement. (a) Laser spot movement on PSD surface caused by micromirror translation; (b) Laser spot movement on PSD surface caused constructively by the translation and tilting of the micromirror

In the measurement, the X-axis of PSD surface is parallel to the mirror plate and the calibration of translation versus the PSD output voltage is calculated. Using above method, the translation of the micromirror is measured to be $135 \mu \mathrm{m}$ at $400 \mathrm{~mA}$, which is only $10 \%$ different from the Zygo 3D profiler's measurement result. However, the Zygo machine cannot measure the dynamic performance of the micromirror. The measured settling time of the micromirror (90\% of the final value for the maximum driving current $400 \mathrm{~mA}$ ), as shown in Figure 2.13, is $16 \mathrm{~ms}$ (micromirror moving downward after current is applied) and $50 \mathrm{~ms}$ (micromirror moving upward after the current is removed) respectively.

The damping caused by the squeeze film effect is significant when the mirror moves to the lower position considering the mirror size of $1.9 \mathrm{~mm} \times 1.9 \mathrm{~mm}$ and the gap is $20 \mu \mathrm{m}$ during the falling period. The damping reduces the oscillation time and that is why the settling time for falling is $16 \mathrm{~ms}$ in Figure 2.13a while the settling time for rising is $50 \mathrm{~ms}$ in Figure 2.13b, when the gap is large and the squeeze film caused damping is not so significant. The oscillation in the transient period in the falling period looks larger than that in the rising period. From the curve in Figure 2.13a, the mirror plate has impossible oscillation beyond the trench bottom from the oscillation magnitude. A possible reason is that the tilting direction in the transient period is not completely aligned with the tilting direction when the mirror settles down, due to the magnetic field generated by the 
charged solenoid. However, the dynamic performance measurement setup and its operations cannot eliminate the tilting after the mirror settles down. Any tilting component unaligned with the tilting direction settling down would cause laser spot displacement on the PSD sensing surface.

There is no deformation or damage before and after bonding. It is noticed that a few hours of dynamic driving $(1 \mathrm{~Hz})$ of the micromirror after bonding is needed to stabilize the structure, i.e. the micromirror moves down by about $10-20 \mu \mathrm{m}$ after a few hours of dynamic driving and then stabilizes. This could be because the contacting supports at the lower corners of each trapezoidal plate become smooth after a few hours' dynamic movement, leading to possible slight lateral movement of the lower corners of the trapezoidal plates, which has significant effect on the central ring height curved up by the residual stress gradient. More research on this is under investigation.

\subsection{Summary}

A micromirror with large translation and high surface quality is presented. The mechanism has the actuation part and the mirror plate fabricated separately to achieve the large translation and high surface quality. Electromagnetic driving is used to realize the large translation with high response speed. A curve-up mechanism is designed to raise the mirror plate up using fabrication induced residual stress gradient. The micromirror is modeled, prototype is fabricated and tested. Experiment results show the micromirror achieves $123 \mu \mathrm{m}$ translation, $2 \mathrm{~nm}$ mirror surface roughness and $15.6 \mathrm{~m}$ radius of curvature of the mirror surface, and settling time of $16 \mathrm{~ms}$ for downward and $50 \mathrm{~ms}$ for upward movement. 


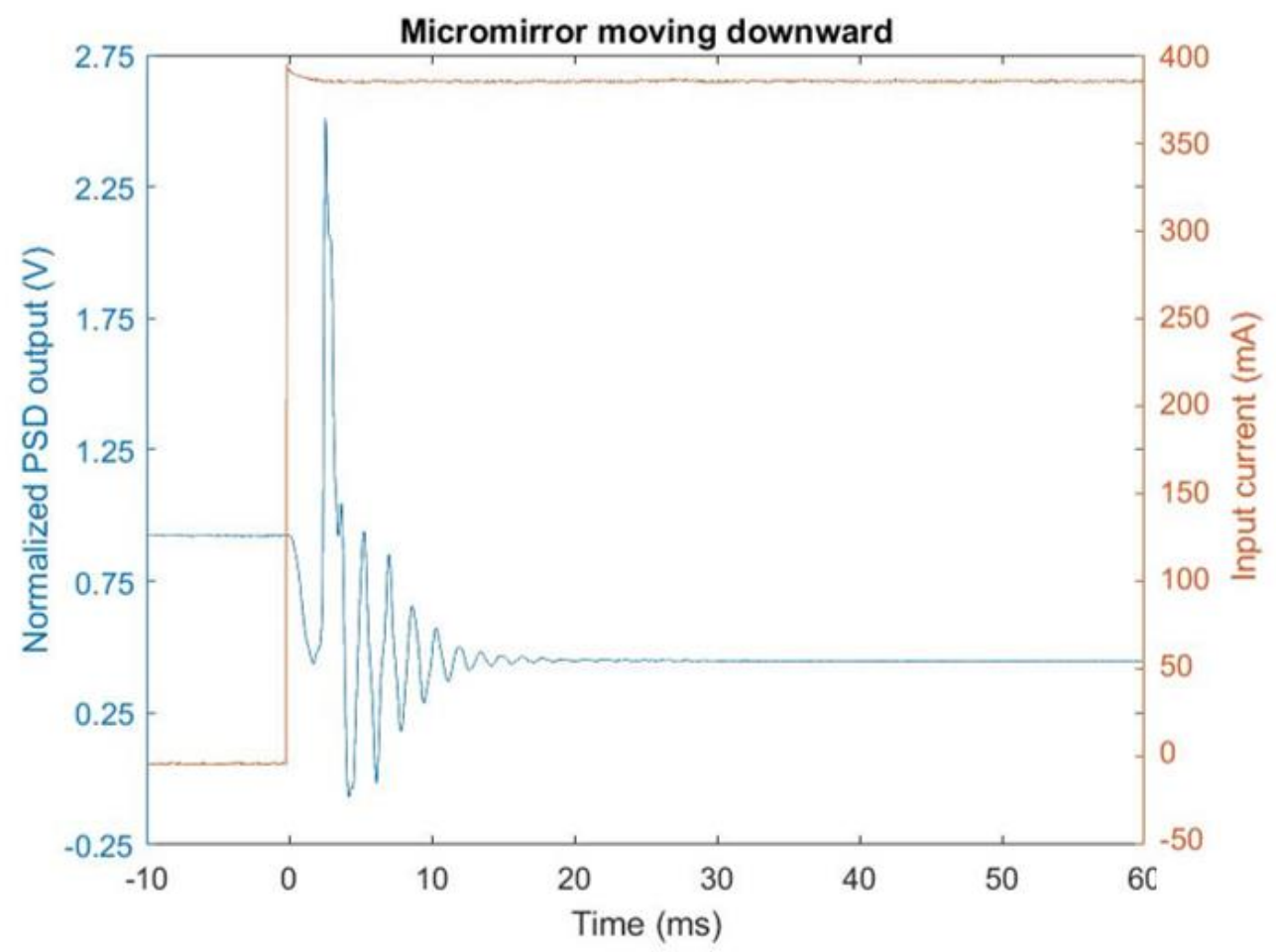

(a)

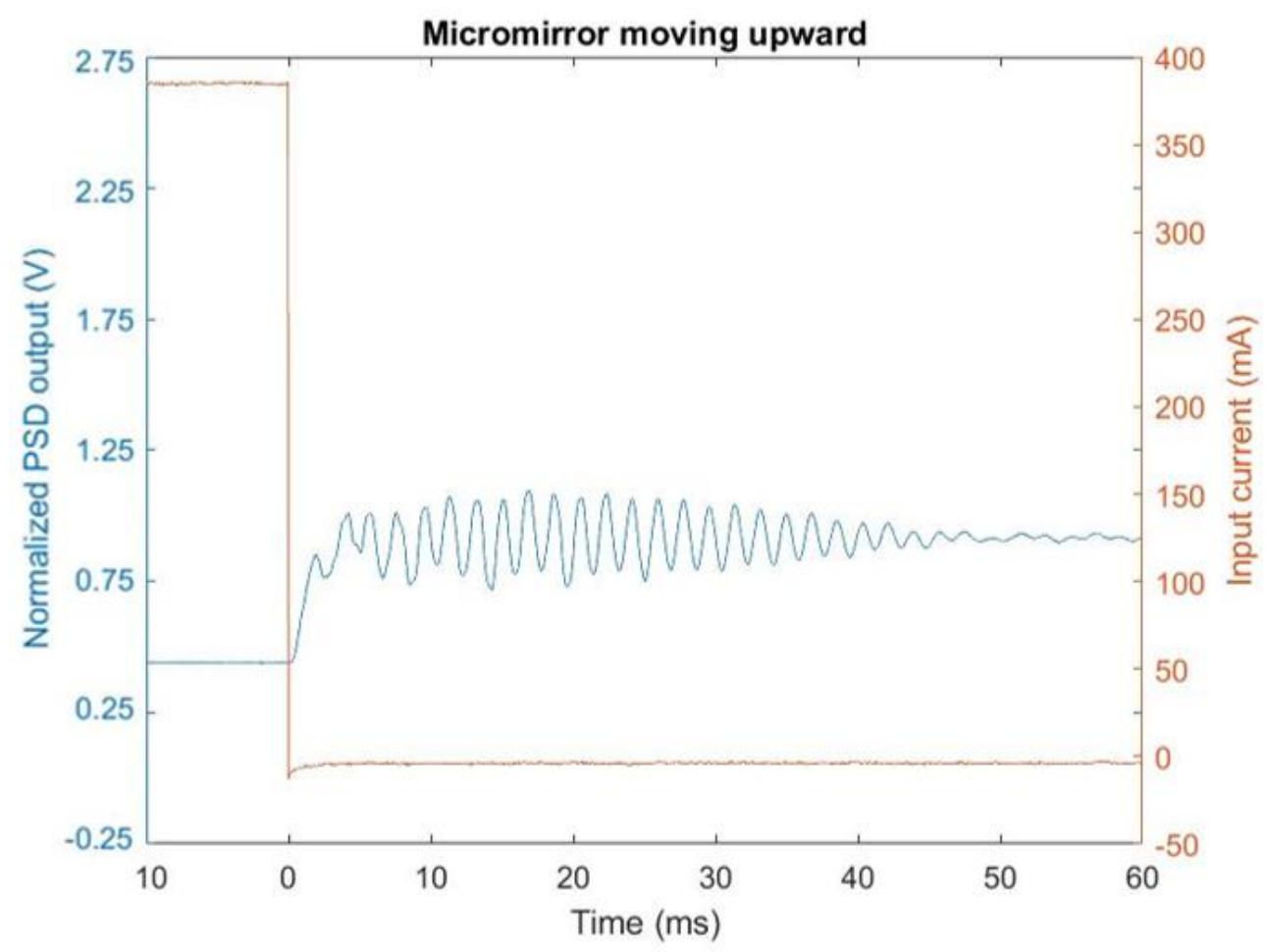

(b)

Figure 2.13 Settling time of the micromirror. (a) Settling time of micromirror downward;

(b) Settling time of micromirror upward 


\section{CHAPTER 3 MAGNETIC FIELD ASSISTED BONDING TECHNOLOGY FOR RELEASED MICRO ACTUATOR AND MIRROR SURFACE}

This chapter is based on the following published paper:

Y. Xue and S. He, "Magnetic field assisted bonding technology for released micro actuator and mirror surface", J. Microsystem Technologies, DOI: 10.1007/s00542-0173432-7, 2017.

A magnetic field pulling-force assisted bonding technology is reported in this chapter according to Objective 2, which is used to bond a mirror plate with a released microactuator using adhesive. This bonding technology is not sensitive to the micromachining process, i.e., any process for microactuator fabrication containing the ferromagnetic structure layer can be used and bonded with a high surface quality mirror plate after released. The conventional wafer bonding technology bonds unreleased actuator with a mirror plate but it is limited to the process compatible with the wafer bonding with a releasing step suitable for wafer bonding. Consequently not all processes (such as those mature and commercially available, and those being able to generate large displacement) can be used to fabricate micromirrors. The bonding technology proposed in this chapter applies non-touching magnetic field pulling-force, instead of conventional compression mechanical force through touching, to bring the microactutor and a mirror plate in contact for bonding such as to avoid: 1) any plastic deformation or damage to the released microactuator; and 2) the risk of sticking the actuator moving part to the substrate due to the adhesive squeezed out from the bonding surfaces. The bonding method is introduced and the critical parameters of the bonding method, i.e., distance between the magnet and the actuator is determined through simulations. A bonding mechanism is built and two different designs are bonded. Bonding results are measured, which verify the bonding method. The bonding is characterized to have a strength of withstanding vibration for a few hours in frequency $20 \mathrm{~Hz}-200 \mathrm{~Hz}$ with $2 \mathrm{~g}-5 \mathrm{~g}$ acceleration and surviving in the temperature of $-30{ }^{\circ} \mathrm{C}$ to $80{ }^{\circ} \mathrm{C}$. After bonding a mirror plate with $15.6 \mathrm{~m}$ of the curvature radius and $2 \mathrm{~nm}$ of the roughness, the bonded micromirror is tested to have a quasi-static displacement of $120 \mu \mathrm{m}$. 


\subsection{Working principle}

The initial position of the mirror plate and the released microactuator are shown in the left of Figure 3.1. Conventionally the mechanical compression force through touching is used to bring the mirror plate in touch with the moving part of the actuator shown in the top right of Figure 3.1. The bonding pressure between bonding surfaces is compression, under which the space between the moving part of the microactuator and substrate is reduced to a few microns or zero. Such that any small amount of adhesive squeezed out from the bonding surfaces will stick the moving part of the actuator to the substrate and cause failure. Due to the imperfections in the mirror plate and microactutor, the bonding mechanism and the motion of bringing the bonding surfaces, undesired lateral force is very often induced, which would damage the released microactuator.

Using the magnetic field force assisted bonding technology, as shown in the bottom right of Figure 3.1, the moving film is attracted downward in the magnetic field generated by the magnet. In the magnetic field shown in the enlarged view in Figure 3.1, an inplane magnetization is formed in the nickel film with the directions of two forces i.e., $F_{N}$ and $F_{S}$ generated at the two ends of each plate, which rotate the plates downward about the anchoring spring. Subsequently the space between the substrate and moving part of the actuator is increased. Such that even a small amount of adhesive squeezed out from the bonding surfaces will not bond the actuator moving part to the substrate since they are not in contact. Furthermore, the magnetic bonding force is uniformly distributed to the whole moving part of the actuator, the undesired lateral force component will not be induced even two bonding surfaces are not perfectly parallel. The magnetic pulling-force as the bonding force is "soft", i.e., it does increase for any lateral obstruction, which is unavoidable due to imperfection in the fabrication of the mirror plate, microactuator and bonding mechanism. While in conventional bonding, the bonding force is executed through external mechanical touch by a probe, which is "hard", i.e., it increases a lot for any lateral obstruction, which could cause damage to the released microactuator.

\subsection{Design and Simulation}

\subsubsection{Bonding mechanism}

The bonding mechanism includes a piece of glass to support the mirror plate and the magnet is moved close to the moving part by a lab jack, as shown in Figure 3.2. The 
mirror plate is placed face-down and the chip holder holds the released microactuator above the mirror plate with a gasket placed between the chip holder and the glass. The chip holder, gasket and actuator are assembled within the alignment structure and fixed on a 3-axis stage. Therefore, the central ring of the moving part of the released microactuator and the mirror plate can be aligned laterally by the stage through a video camera' monitoring, as shown in Figure 3.2a. After they are aligned, the video camera is replaced by a lab stage with a magnet on it, which raises the magnet to attract the moving part of the miroactuator down and in touch with the mirror plate, as shown in Figure 3.2b. And the zoom in image of inside of the alignment structure is shown in Figure 3.2c. The dimensions of the bonding mechanism are listed in Table 3.1.

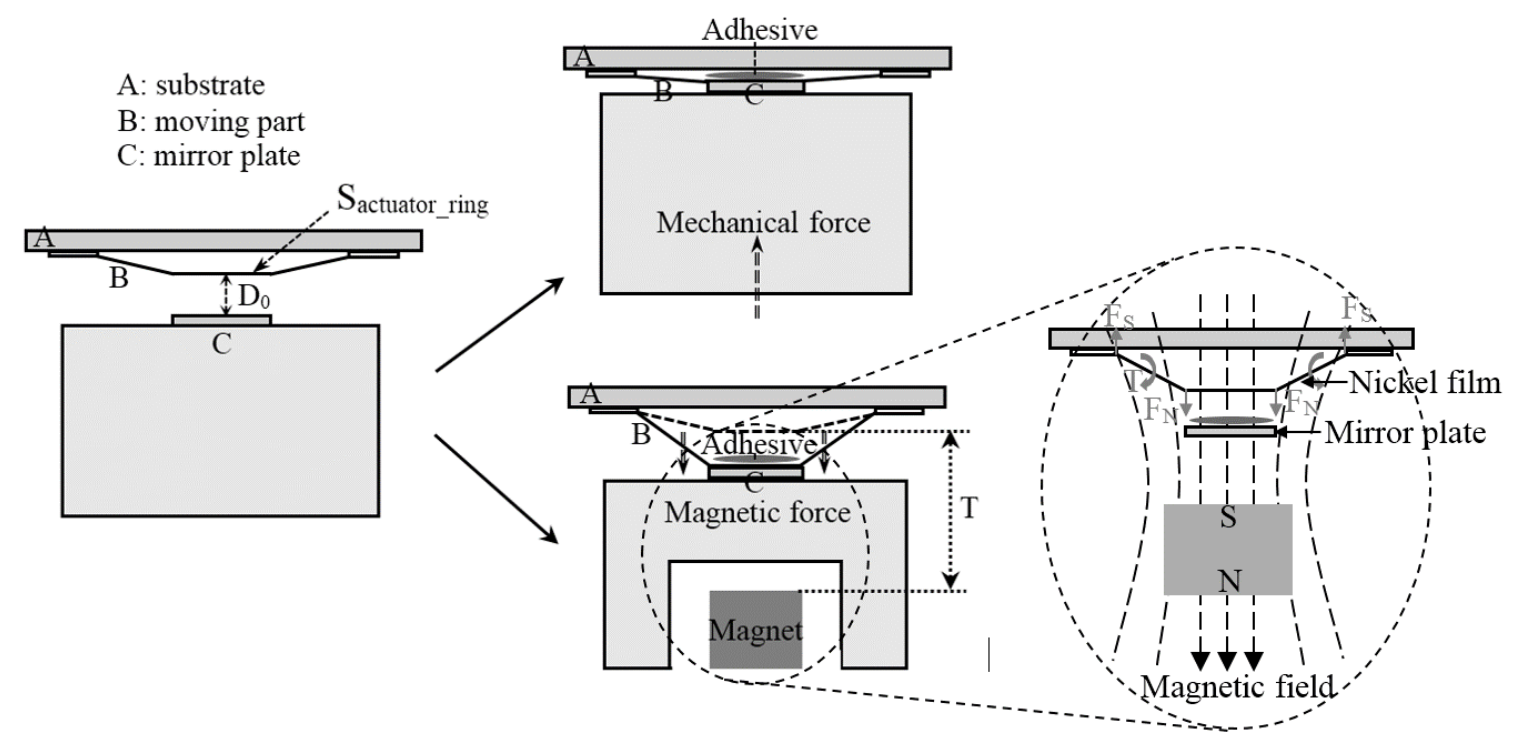

Figure 3.1 Released miroactuator and mirror plate before bonding (left); Conventional bonding mechanical using compression force (top right); and Novel bonding mechanism using magnetic field pulling-force (bottom right) 


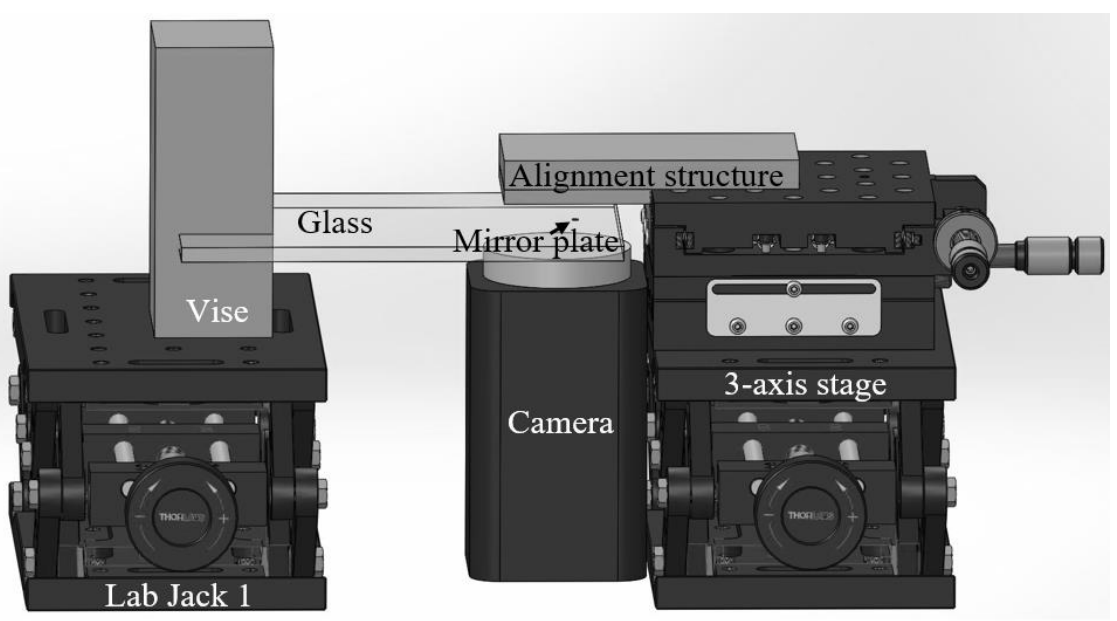

(a)

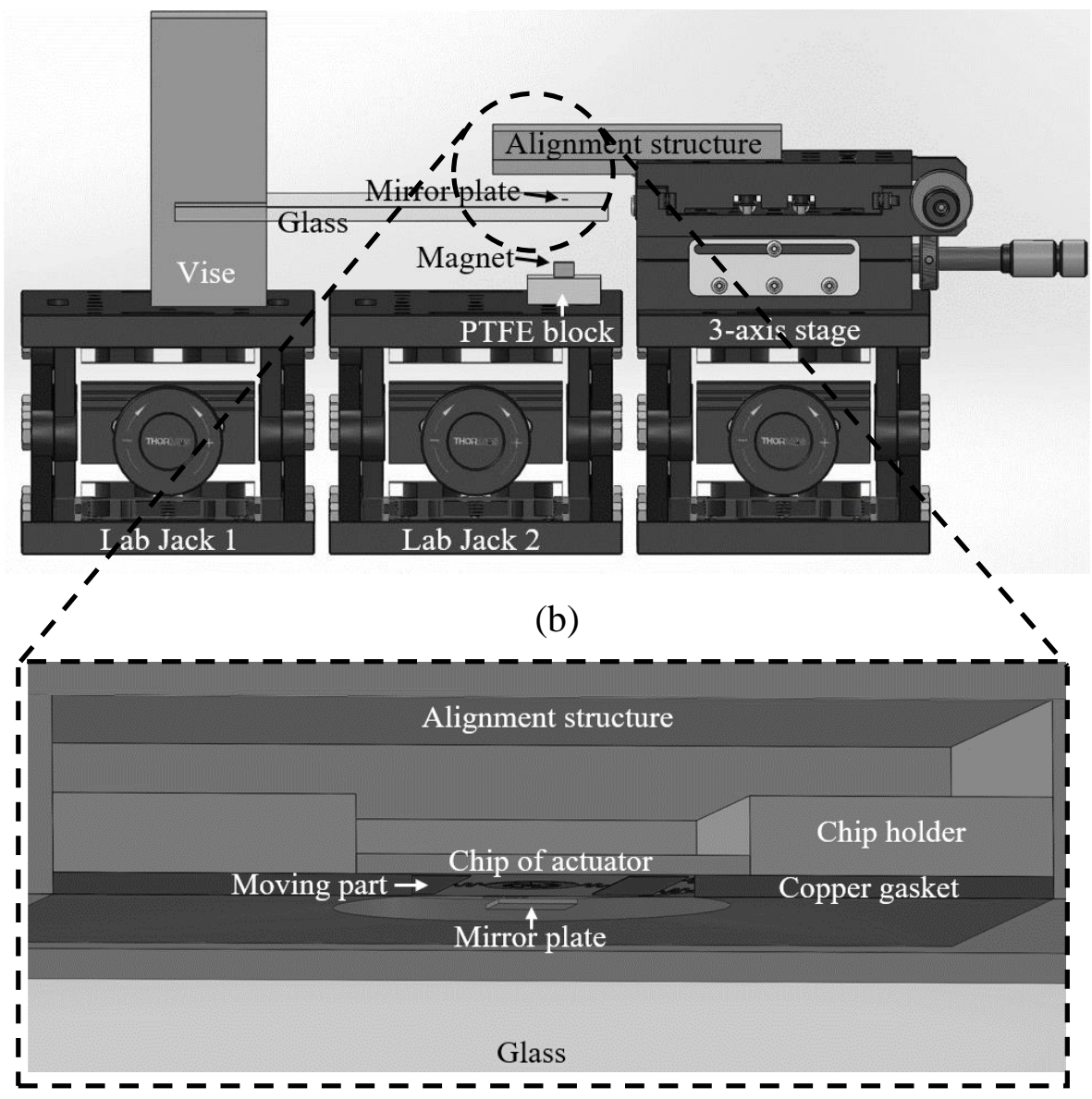

(c)

Figure 3.2 The bonding mechanisms. (a) Bonding mechanism with the video camera under the mirror plate; (b) Bonding mechanism with a stage and magnet replacing the video camera; (c) Zoom in image of the alignment structure 
Table 3.1 Dimensions of the bonding mechanism

\begin{tabular}{|c|c|c|}
\hline Part name & \multicolumn{2}{|c|}{ Dimension (mm) } \\
\hline $\begin{array}{c}\text { Alignment structure } \\
\text { External/ Internal })\end{array}$ & $50(\mathrm{~L}) \times 50(\mathrm{~W}) \times 10(\mathrm{H})$ & $25.4(\mathrm{~L}) \times 25.4(\mathrm{~W}) \times 10(\mathrm{H})$ \\
\hline $\begin{array}{c}\text { Chip Holder } \\
\text { External/ Internal })\end{array}$ & \begin{tabular}{c}
$25.4(\mathrm{~L}) \times 25.4(\mathrm{~W}) \times 5(\mathrm{H})$ \\
\hline $\begin{array}{c}\text { Gasket } \\
(\text { External/Internal })\end{array}$
\end{tabular} & $\begin{array}{c}25.4(\mathrm{~L}) \times 25.4(\mathrm{~W}) \times 0.55 \\
(\mathrm{H})\end{array}$ \\
\hline $\begin{array}{c}\text { Mirror Plate } \\
(\mathrm{P} 1 / \mathrm{P} 2)\end{array}$ & $1.9(\mathrm{~L}) \times 1.9(\mathrm{~W}) \times 0.35(\mathrm{H})$ & $1.9(\mathrm{~L}) \times 1.9(\mathrm{~W}) \times 0.12(\mathrm{H})$ \\
\hline Glass & \multicolumn{2}{|c|}{ Thickness: 5mm, length/width $>50 \mathrm{~mm}$} \\
\hline
\end{tabular}

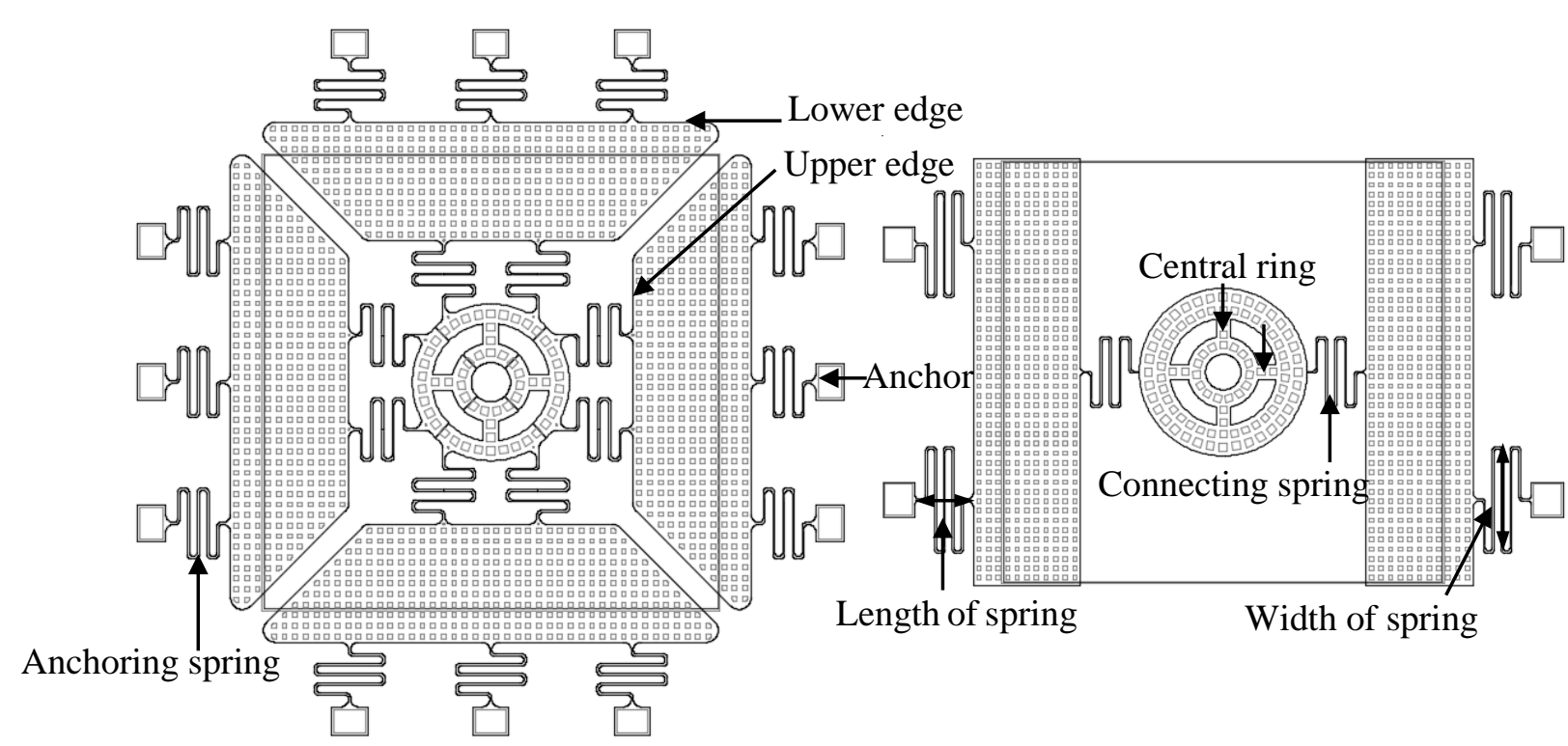

(a)

(b)

Figure 3.3 Microactuators for bonding. (a) Design 1; (b) Design 2 
Table 3.2 Dimensions of the microactuators

\begin{tabular}{|c|c|c|}
\hline Part name & Microactuator of design 1 & Microactuator of design 2 \\
\hline Upper edge of plate & $3 \mathrm{~mm}$ & $6 \mathrm{~mm}$ \\
\hline Lower edge of plate & $6 \mathrm{~mm}$ & $6 \mathrm{~mm}$ \\
\hline Height of plate & $1.5 \mathrm{~mm}$ & $1.5 \mathrm{~mm}$ \\
\hline $\begin{array}{c}\text { Actuator moving part } \\
\text { thickness }\end{array}$ & $20 \mu \mathrm{m}$ & $20 \mu \mathrm{m}$ \\
\hline Central ring & $2 \mathrm{~mm}($ diameter $)$ & $2.4 \mathrm{~mm}($ diameter $)$ \\
\hline Connection spring & $0.9 \mathrm{~mm}(\mathrm{~L}) \times 0.8 \mathrm{~mm}(\mathrm{~W})$ & $0.8 \mathrm{~mm}(\mathrm{~L}) \times 1 \mathrm{~mm}(\mathrm{~W})$ \\
\hline Anchor spring & $0.8 \mathrm{~mm}(\mathrm{~L}) \times 0.9 \mathrm{~mm}(\mathrm{~W})$ & $0.8 \mathrm{~mm}(\mathrm{~L}) \times 1.5 \mathrm{~mm}(\mathrm{~W})$ \\
\hline
\end{tabular}

\subsubsection{Simulation of distance between magnet and moving part of microactuator}

Two microacutators, i.e., design 1 and design 2, as shown in Figure 3.3, are to be bonded with mirror plates to verify the bonding technology proposed in this chapter. Design 1 is described in Chapter 2 and [90]. Design 2 is a 1-axis rotation actuator. Like design 1, the moving part of design 2 is curved up by the residual stress gradient in the nickel film and contacts with the substrate through the lower corners of the plates (and/or points on the anchoring springs in some prototypes since both the lower corners of the plates and anchoring spring are very close/touching the substrate). Design 2 has much lower stiffness than that of design 1 . Table 3.2 lists the dimensions of design 1 and design 2.

Basing on the working principle of the bonding technology, the moving part of the microactuator needs to touch the mirror plate under the magnetic field pulling-force without causing any plastic deformation. The following parameters are selected to ensure the above criteria are met: A) Permanent magnet parameters including its size and magnetic strength; $B$ ) The distance $\left(\mathrm{D}_{0}\right)$ between the central ring surface of the microactuator's moving part (named as $\mathrm{S}_{\text {actuator_ring here after) and mirror plate when no }}$ magnetic force; and C) The distance $(\mathrm{T})$ between the magnet top surface to $\mathrm{S}_{\text {acturtor_ringe. }}$ $\mathrm{S}_{\text {acturtor_ringe, }} \mathrm{D}_{0}$ and $\mathrm{T}$ are marked in Figure 3.1. These parameters are chosen as follows. A) Determine permanent magnet 
The permanent magnet is chosen to be smaller than the microactuator chip, e.g., $7 \mathrm{~mm}$ x $7 \mathrm{~mm}$ x $5 \mathrm{~mm}$ with coercive force of $9.25 \mathrm{E} 5 \mathrm{~A} / \mathrm{m}$ and residual induction of 1.192 Tesla. B) Determine $\mathrm{D}_{0}$

The $\mathrm{D}_{0}$ has a wide range as long as it will not cause the maximum stress in the moving part beyond the yield strength. According to a large number of simulations on Design 1 and Design 2, the range of approximately $>0 \mu \mathrm{m} \sim 700 \mu \mathrm{m}$ can be used for both designs

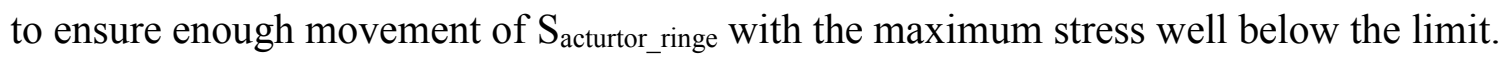
The final value of $\mathrm{D}_{0}$ for design 1 is chosen to be $200 \mu \mathrm{m}$ and $280 \mu \mathrm{m}$ for design 2 , according to the above range and available copper gasket and height adjusting parts. C) Determine $\mathrm{T}$

After the magnet parameters and $\mathrm{D}_{0}$ are chosen, the $\mathrm{T}$ is determined to meet the following two criteria: 1$)$ The moving displacement ( $D_{\text {moving }}$ ) of the surface of $S_{\text {acturtor_ringe }}$ under the permanent magnet, assuming no mirror plate blocks the movement, is larger than $\mathrm{D}_{0}$; and 2) The maximum stress in the moving part of the actuator is less than the yield strength. The following sub-steps are followed to determine T.

Simulations are performed using SolidWorks ${ }^{\circledR}$ [148], Ansys Multiphysics ${ }^{\circledR}$ [145] and CoventorWare ${ }^{\circledR}$ when the variable $\mathrm{D}_{0}$ and parameter of magnet are determined.

C_1) The shape and positon of the moving part of the microactuator after releasing due to residual stress gradient is obtained by simulations using the software of CoventorWare and similar method of [90] with the nickel elastic modulus of $159 \mathrm{GPa}$ and an average residual stress gradient of $-4.72 \mathrm{MPa} / \mu \mathrm{m}$ [144].

C_2) A model of the moving part of the microactuator is created in SolidWorks basing on the results obtained in $\mathrm{C}_{-} 1$ with the permanent magnet in distance $\mathrm{T}$ chosen within the range of $3 \mathrm{~mm} \sim 30 \mathrm{~mm}$ which is well wide enough to cover the possible values of T. The model is imported into the Magneto-Static simulation module of Ansys Multiphysics to simulate the magnetic force acting on the moving part of the microactuator in the magnetic field. Figure 3.4a shows the distribution of magnetics flux density in design 1 and design 2 with T equal to $6 \mathrm{~mm}$ and $10 \mathrm{~mm}$ respectively. The T values in Figure 3.4a are selected after a few simulations with $\mathrm{T}$ varying in its range.

C_3) The obtained magnetic force is imported to CoverntorWare MemMech module to simulate the deformation of the moving part of the microactuator. 

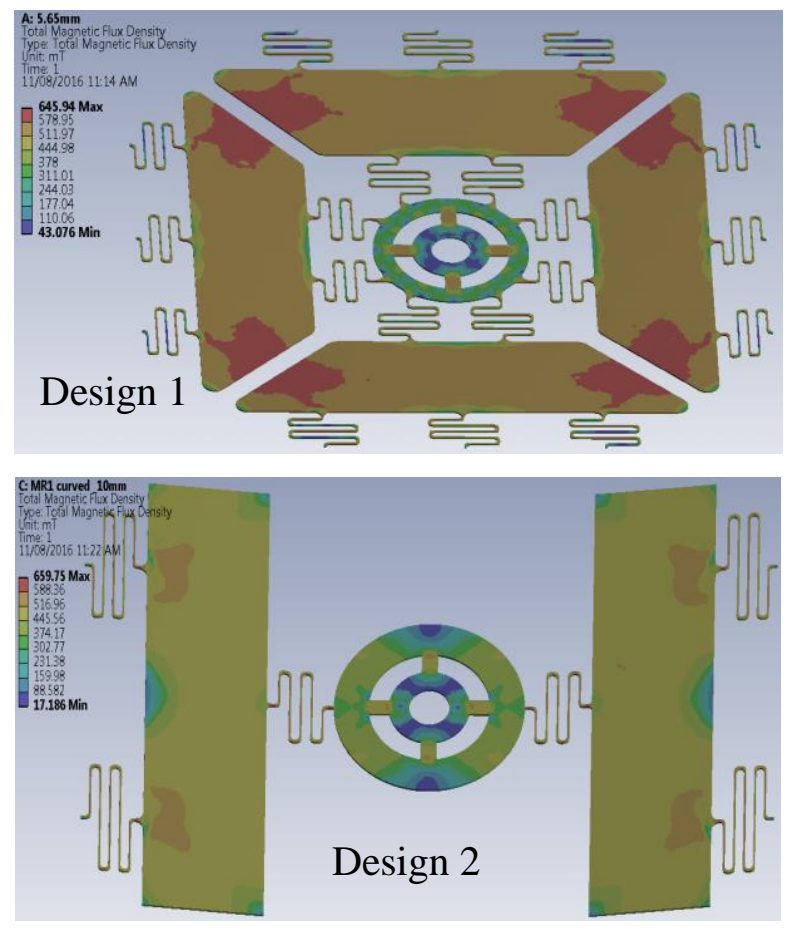

(a)

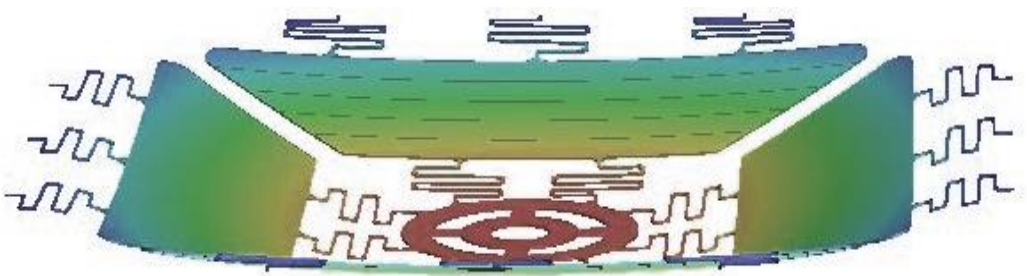

Design 1
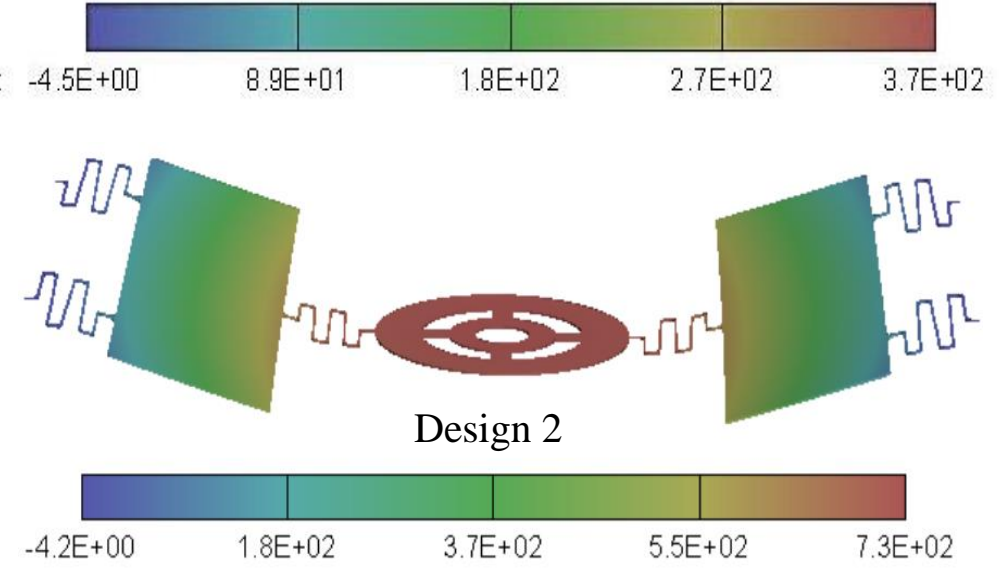

(b)

Figure 3.4 Magnetic and deformation simulations for design 1 and design 2. (a)

Distribution of magnetic field in design 1 and design 2; (b) Displacement ( $\left.\mathrm{D}_{\text {actuator_ring }}\right)$ of design 1 and design 2 
To obtain accurate T value, a few iterations are needed, as used in Chapter 2 and [90]. The iterations normally would lead to $<20 \%$ change of the value in comparison to the value before iteration. However the $\mathrm{T}$ is determined in such way that the two above criteria are met with a very large safety margin $(>70 \%)$, no time consuming iteration is needed.

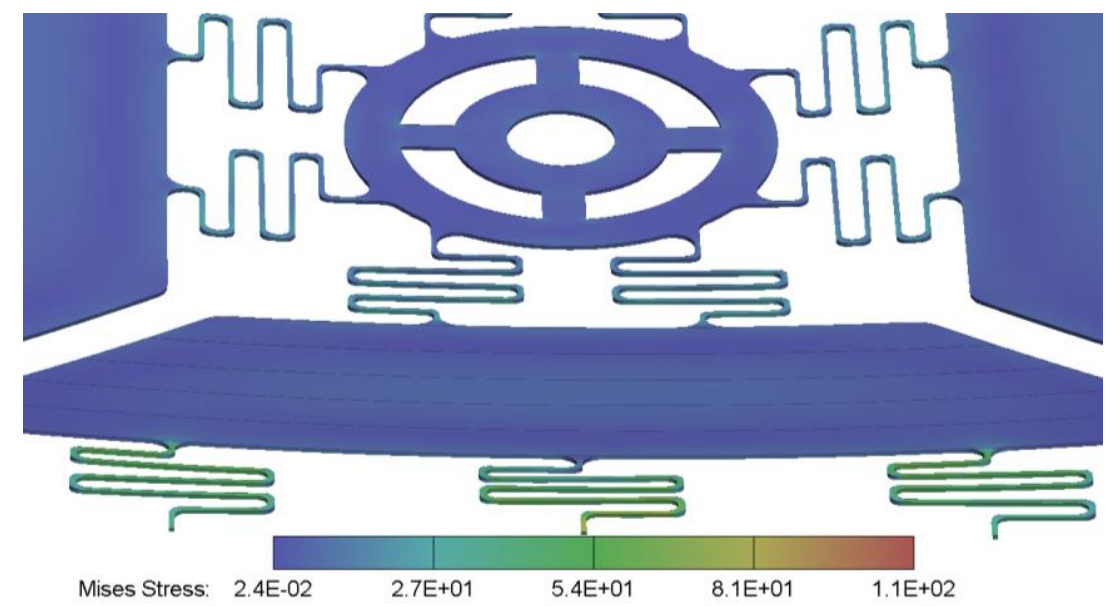

(a)

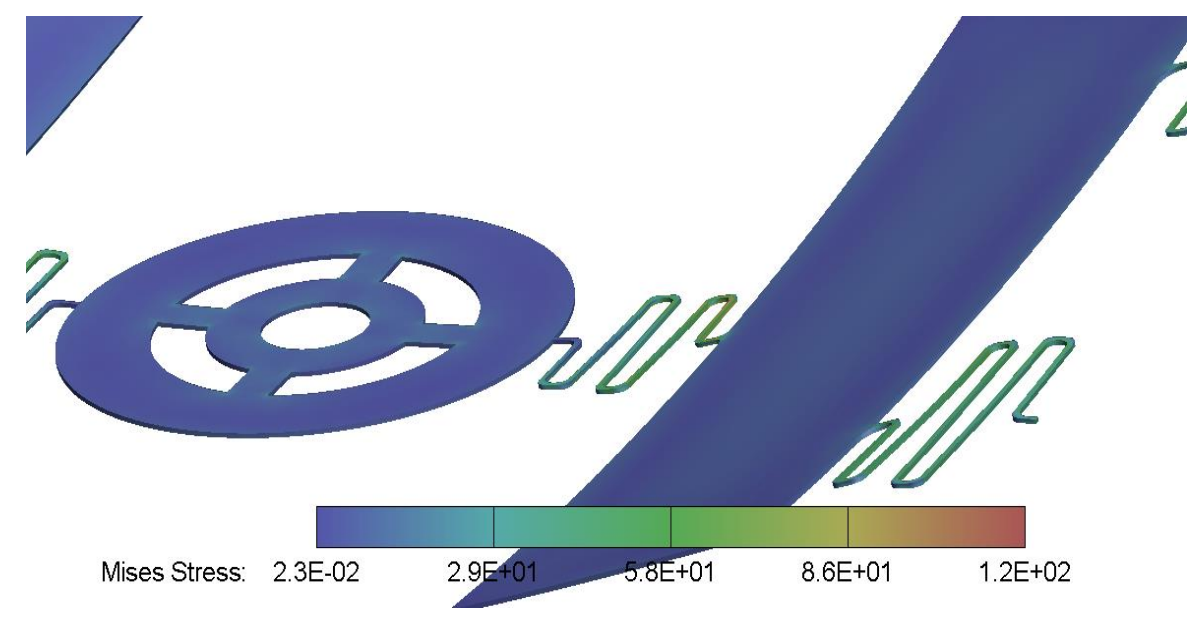

(b)

Figure 3.5 Stress in designs 1 and 2 when displacement is $\mathrm{D}_{0}$. (a) Maximum Von Mises stress of $107 \mathrm{MPa}$ in design 1; (b) Maximum Von Mises stress of $115 \mathrm{MPa}$ in design 2

Using above method the $T$ and $D_{\text {moving }}$ for design 1 are $6 \mathrm{~mm}$ and $300 \mu \mathrm{m}$, for design 2 are $10 \mathrm{~mm}$ and $700 \mu \mathrm{m}$ respectively, as shown in Figure $3.4 \mathrm{~b}$. $\mathrm{D}_{\text {moving }}$ is the displacement assuming no mirror plate blocks the moving part of the microactuator. In the bonding process the displacement of the ring of the microactuator is $\mathrm{D}_{0}$ considering the mirror plate blocking the movement. In order to find out the maximum stress in the moving part 
with the displacement of $\mathrm{D}_{0}$, a force on the central ring in the upward direction is applied to limit the downward translation of the ring to $\mathrm{D}_{0}$ and find out the maximum stress in CoventorWare simulations. The results are shown in Figure 3.5, the maximum Von Mises stress is $107 \mathrm{MPa}$ in design 1 and $115 \mathrm{MPa}$ in design 2, both of which are smaller than the yield strength $400 \mathrm{MPa}$ [149] with a safety margin of $>70 \%$.

\subsection{Experiment}

\subsubsection{Prototypes of actuators of design 1 and design 2}

The prototypes of the microactuators of design 1 and design 2 are fabricated using MetalMUMPs. The mirror plates are fabricated by simply dicing a polished silicon wafer with $100 \mathrm{~nm}$ aluminum coated. A Zygo 3D profiler machine is used to measure the height of the moving part and the surface quality of the mirror plate. Because the microactuator (20 $\mu \mathrm{m}$ nickel plus $0.5 \mu \mathrm{m}$ gold on its top) fabricated using MetalMUMPs has poor surface roughness, the measured surface is noisy. If low magnification lens is used, the whole actuator can be covered in one scan, but the result cannot be accurately extracted, as shown in Figure 3.6a. When the high amplification lens is used only a small area can be scanned, as shown in Figure 3.6b. Therefore points on the actuator surface are measured separately to construct cross section profile along line AB in Figure 3.6a with the same reference surface, which is the trench bottom. It is found the measured value could vary as high as $\pm 1 \mu \mathrm{m}$ for multiple mountings of the same chip when the measurement height is in the range of several hundred micrometers, even the optical 3D profiler is of high resolution. This might be because: 1) The mounting of the microactuator chip on the stage is not perfectly and consistently perpendicular to the lens; and 2) The rough surface of the actuator makes the height extraction inaccurate if the actuator's surface (not the bonded mirror plate) is measured. 

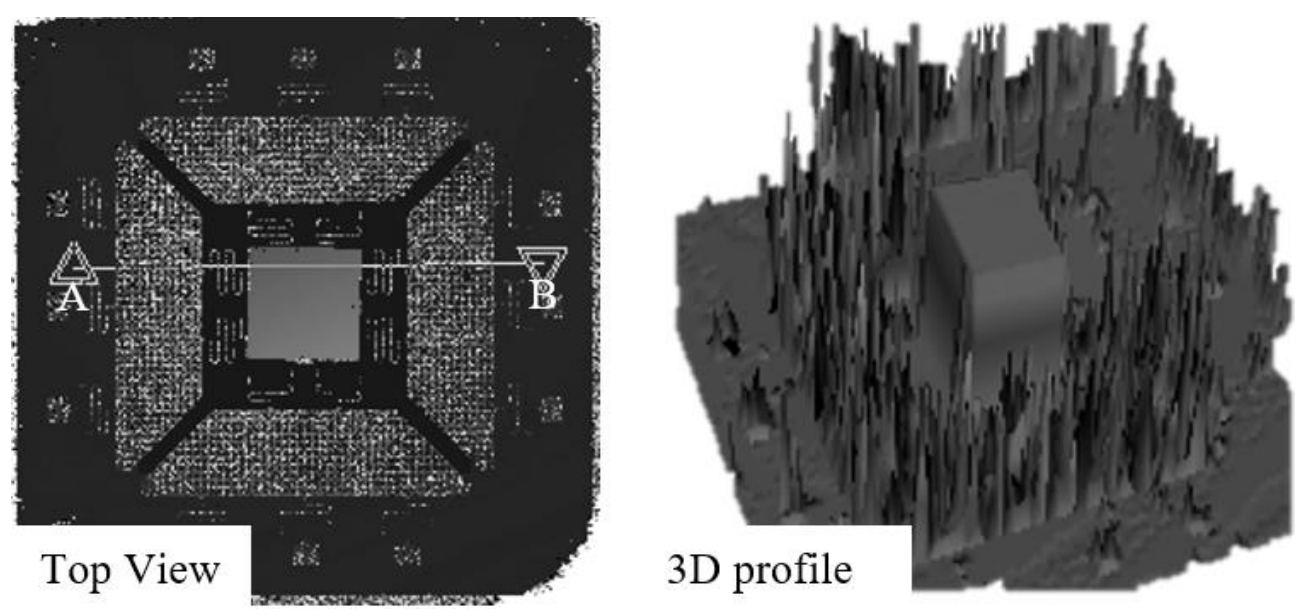

(a) .
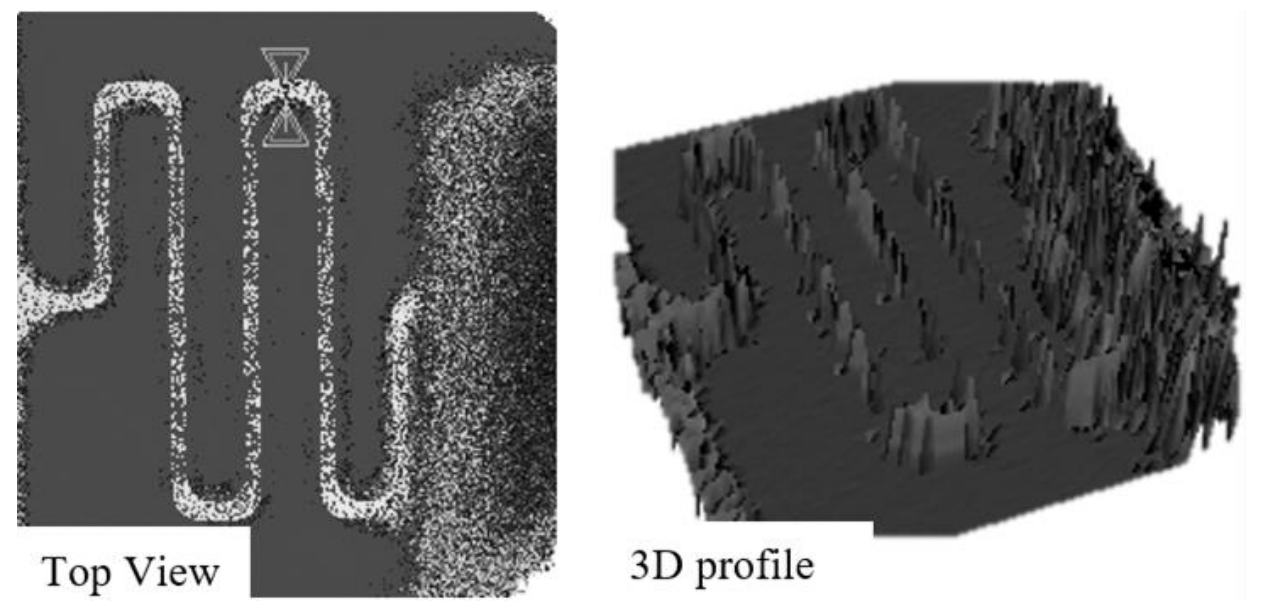

(b)

Figure 3.6 Measuerment under 3D optical profiler. (a) Measurement using low magnification lens; (b) Measurement using high magnification lens

\subsubsection{Bonding process}

The bonding mechanism is shown in Figure 3.7 and bonding process is described as follows.

1. The adhesive (Loctite 312 [150]) is mixed with the activator (LOC736 [151]) at room temperature and applied to the backside of the mirror plate, as shown in Figure 3.8a. The ratio of adhesive to activator is about 2 with the waiting time of $>2$ hours after the mixing. A piece of thin plastic film can be used to adjust the gap between the moving part of the microactutor and the mirror plate. 
2. The released microactuator is positioned on an aluminum cylinder using a small piece of double sided tape $\left(2 \sim 3 \mathrm{~mm}^{2}\right)$, as shown in Figure 3.8b. It is then put in the alignment structure with the chip holder and gasket through operating the cylinder as shown in Figure 3.8c, both of which are fastened by 4 screws. Since the microactuator chip is constrained laterally and downwardly, a slight twist of the cylinder will separate the cylinder from the microactuator chip.

3. After the aluminum cylinder is removed, the alignment structure is fixed on the 3-axis stage with the actuator as shown in Figure 3.8d. The central ring of the moving part of the microactuator and the mirror plate are aligned by moving the stage holding the mirror plate with the video camera's monitoring through the glass.

4. A lab jack replaces the video camera and adjusts the height of the permanent magnet to control the gap between the magnet and the microactuator to the value simulated in section 3.2.2, as shown in Figure 3.8e. In order to avoid the adhesive squeezed out of the bonding surface from flowing to the glass surface, the magnet is removed a few seconds after the mirror plate and the moving part of the microactutor touch.

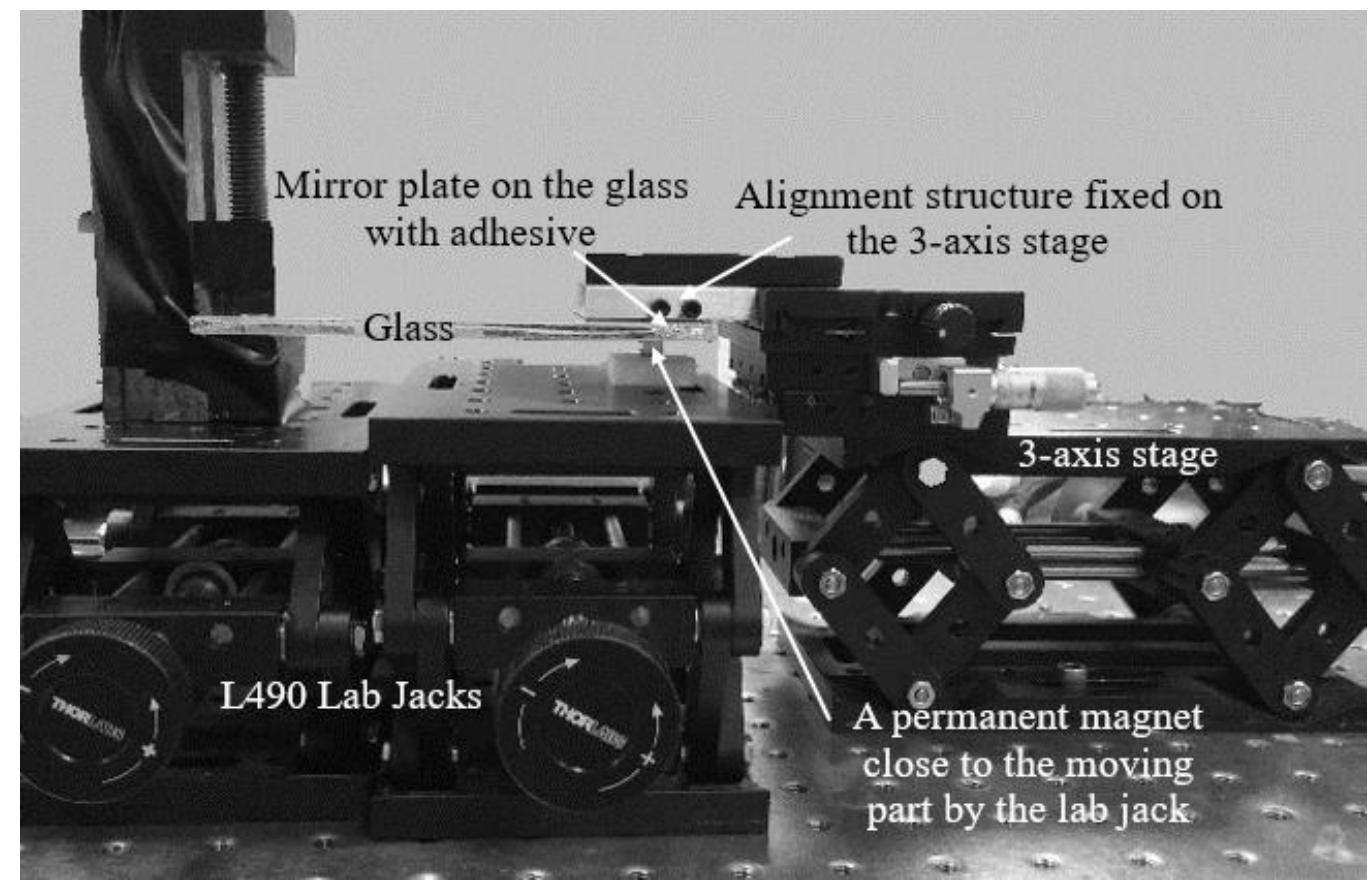

Figure 3.7 Assembled bonding mechanism 


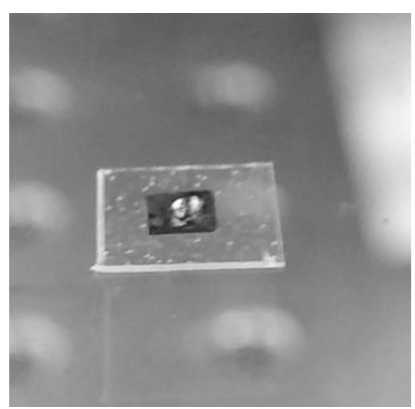

(a)

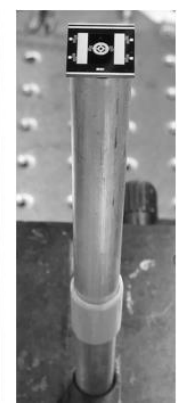

(b)

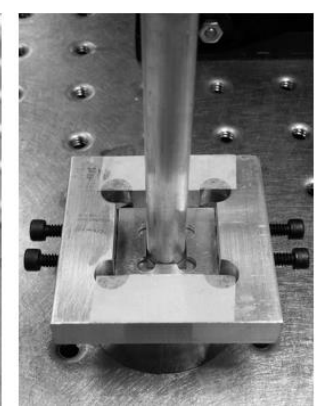

(c)

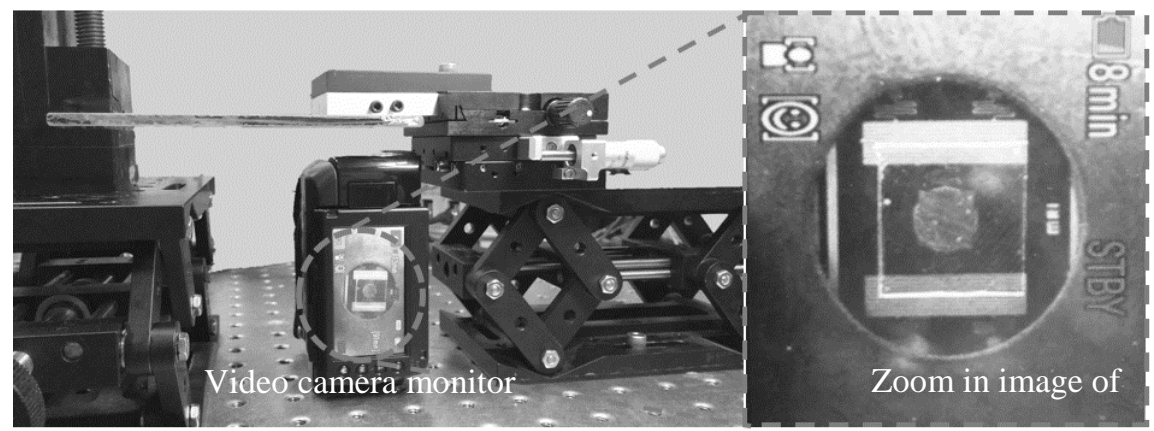

(d)

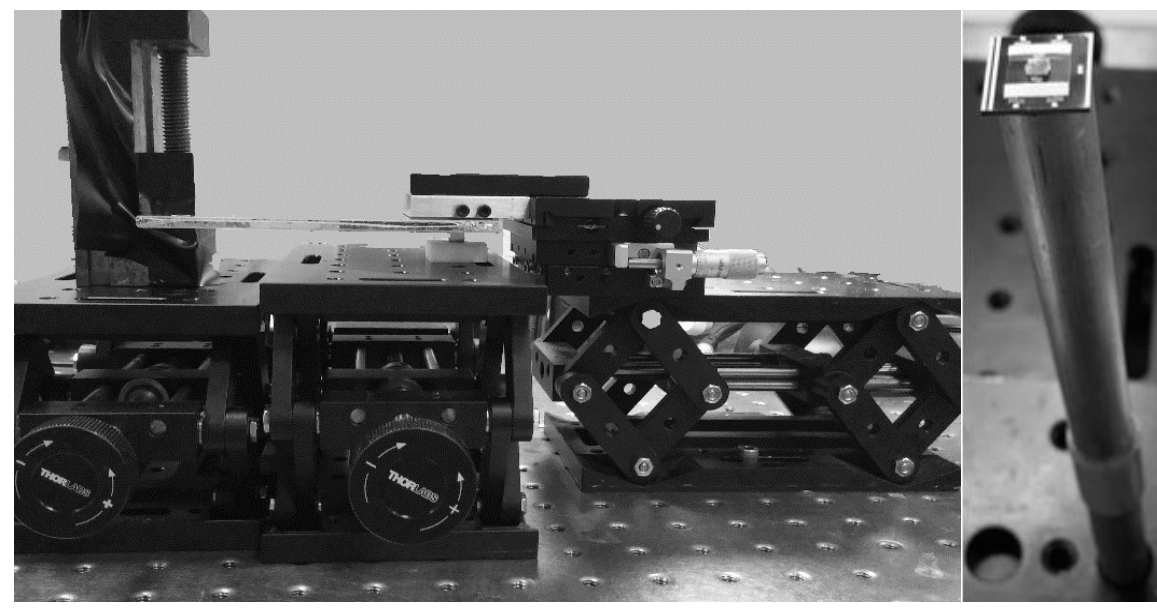

(e)

(f)

Figure 3.8 Bonding process. (a) Adhesive is applied to the backside of the mirror plate;

(b) The microactuator is positioned on the aluminum cylinder; (c) The microactutor is put in the chip holder and fixed on the alignment structure; (d) The central ring of the moving part and the mirror plate are laterally aligned to ensure the mirror plate on the center of the ring by the 3 -axis stage and monitored by a video camera; (e) The permanent magnet is raised up by a lab jack to pull the moving part of the microactuator down to touch the mirror plate; (f) After curing the adhesive, the microactuator with the mirror plate bonded is taken out by the aluminum cylinder 
5. The 3-axis stage is raised up to lift the mirror plate from the glass a few minutes (let the adhesive and the two bonding surfaces thoroughly touch) after the magnet is removed.

6. The microactuator bonded with the mirror plate is kept on the chip holder with the mirror plate facing down for 12 hours to cure the adhesive.

7. After the adhesive is completely cured, the micoractuator with the mirror plate bonded is taken out by the aluminum cylinder as shown in Figure 3.8f.

\subsubsection{Bonding results}

The design 2 is bonded with a $1.9 \mathrm{~mm} \times 1.9 \mathrm{~mm} \times 0.12 \mathrm{~mm}$ mirror plate following the above bonding procedure and using the above bonding mechanism. Design 1 is bonded with a $1.9 \mathrm{~mm} \times 1.9 \mathrm{~mm} \times 0.35 \mathrm{~mm}$ mirror plate using the same bonding method and similar mechanism, in which the video camera for lateral alignment is not used. Instead, grid lines drawn on the PTFE block are used for lateral alignment to try the lower cost mechanism. It is proved that the video camera lateral alignment method is more convenient with higher alignment accuracy. Figure 3.9 is the SEM image of design 1 bonded with the mirror plate. The section profiles along line AB (in Figure 3.6a) before and after bonding are shown in Figure 3.10. Figure 3.10 shows the profile along line AB after bonding matches the shape of the profile along the same line $\mathrm{AB}$ except that it is slightly lower, which is caused by the gravity of the mirror plate. In order to find out the downward displacement caused by the mirror plate, the following experiment is conducted. A mirror plate is dropped onto the bonded mirror plate from the place of 1 $\mathrm{mm}$ above. This method does not require any external touch to the released microactuator except for the mirror's gravity, which does not induce any plastic deformation to the miroactuaror. Of course no lateral alignment to ensure the mirror plate is in the center of the bonded mirror plate. This method cannot be used for bonding because: 1) No lateral alignment to ensure the mirror plate in the center of the ring; and 2) The adhesive will leak from the hole (needed for releasing process) of the ring to stick the ring to the substrate or the trench bottom if this dropping method is used for bonding. Measurements show the mirror plate causes the central part of the released microactuaror to move downward by $\sim 2 \mu \mathrm{m} .40$ points on the moving part of the microactutor are measured before and after the bonding, which are marked as red dots in Figure 3.11a. Their heights 
with the reference of the trench bottom under the moving part are measured and the change before and after the bonding are shown Figure 3.11b. It shows that the 4 points on the connection spring are down by $\sim 1.7 \mu \mathrm{m}$ after bonding due to the mirror plate gravity, which is very close to the move-down value due to the mirror plate's gravity ( $\sim 2 \mu \mathrm{m})$. The discrepancy is well within the measurement variation due to different mountings.

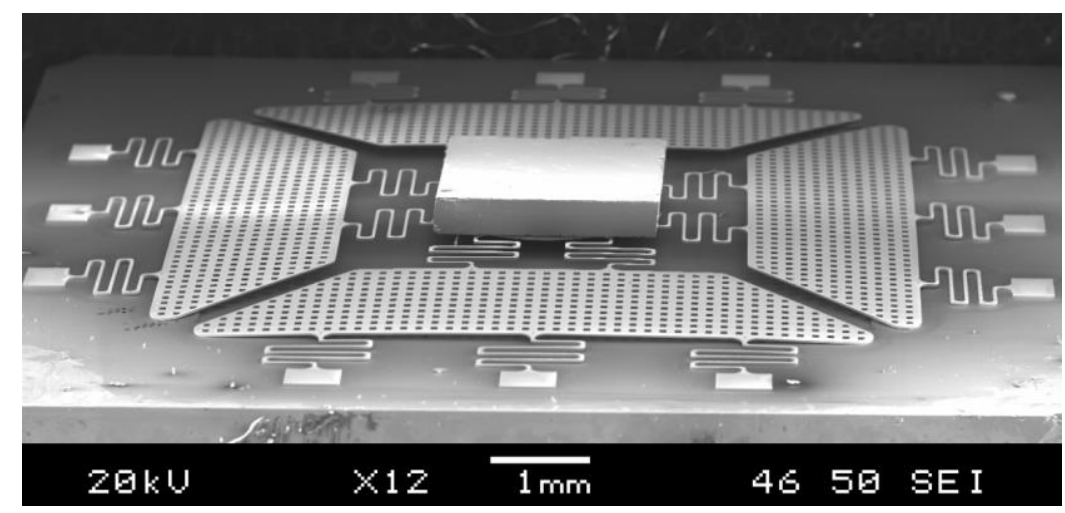

Figure 3.9 Design 1 with mirror plate bonded

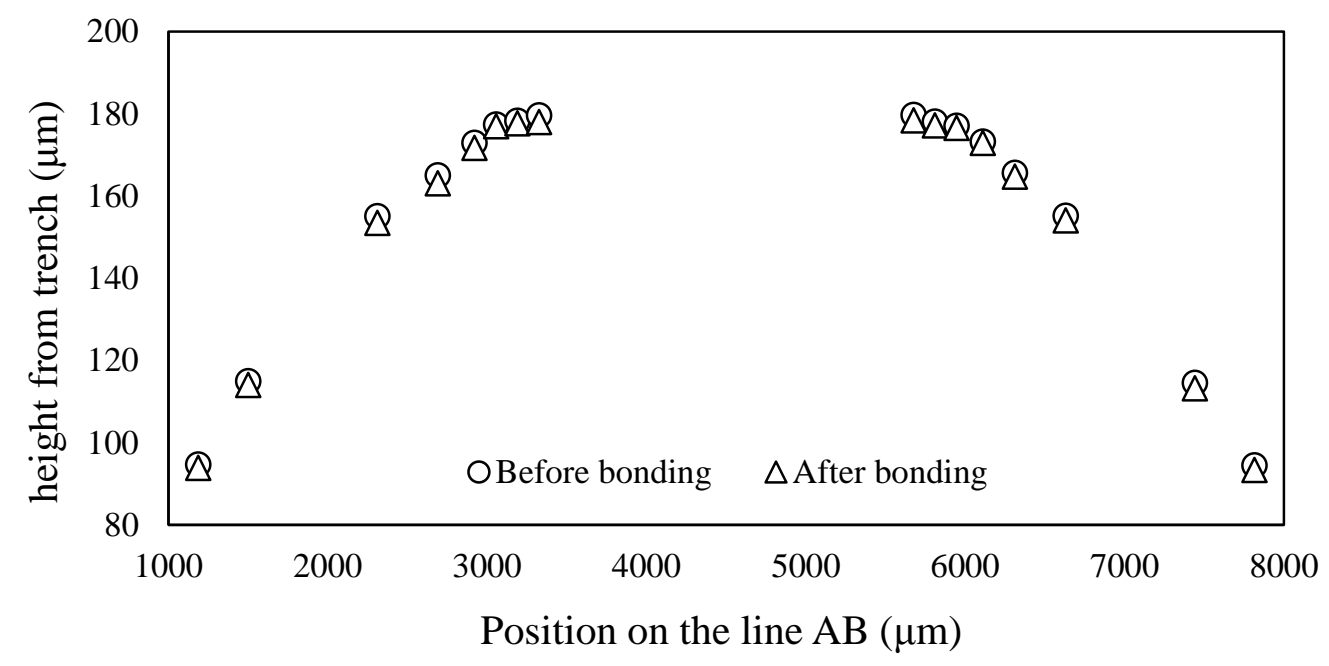

Figure 3.10 Cross section profiles along lines $\mathrm{AB}$ (as shown in Figure 3.6) before and after bonding 


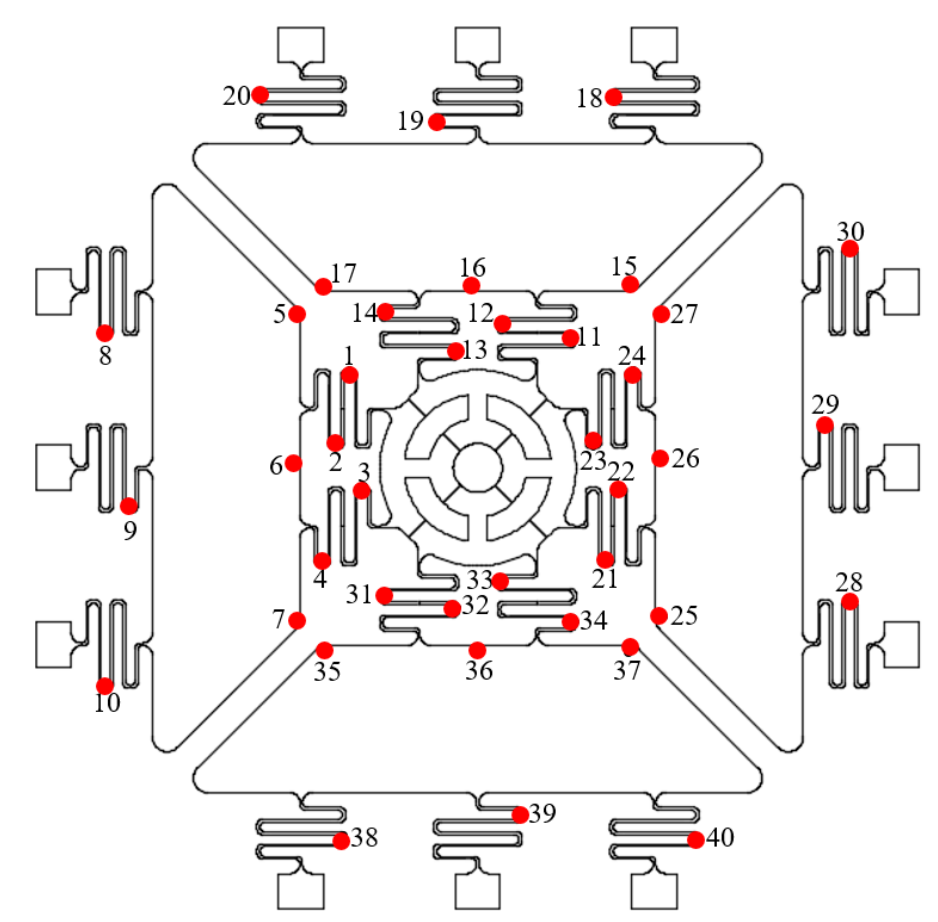

(a)

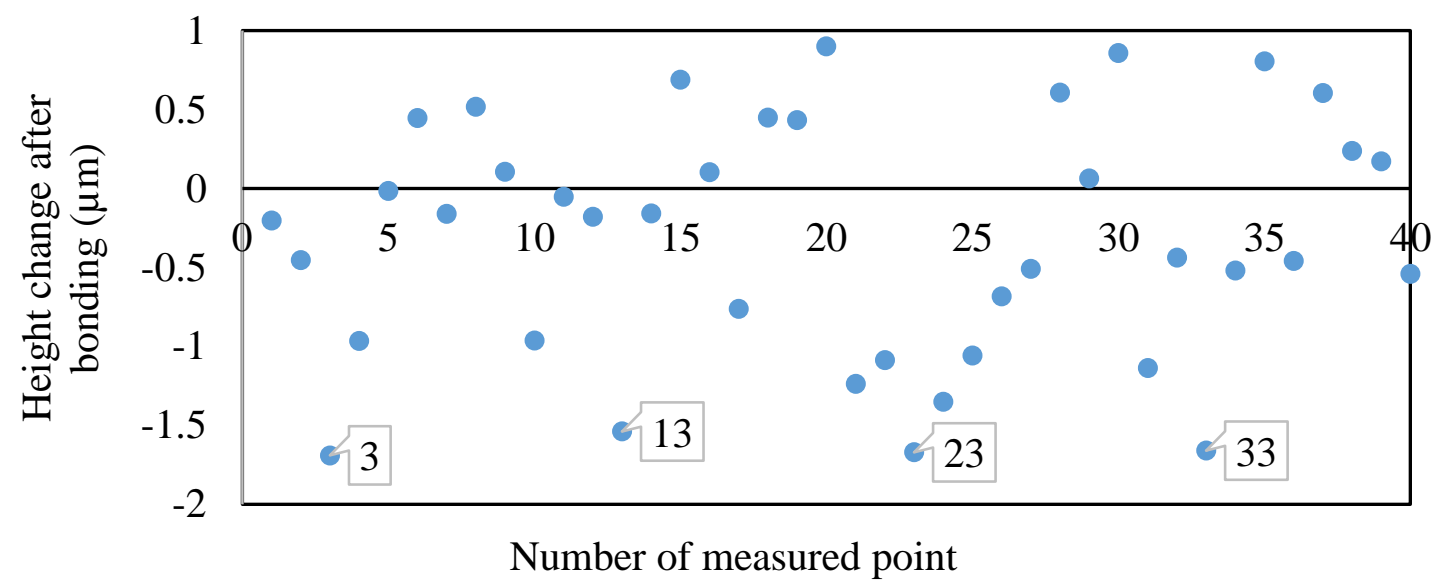

(b)

Figure 3.11 Measurement result of design 1 after bonding. (a) Points measured on design 1; (b) Height change after bonding

It should be noted that the mirror plate's orientation is not required since the orientation does not affect the application of translation micromirror. The lateral alignment in the bonding procedure is to ensure the mirror plate is on the center of the ring of the microactuator. As mentioned in [90], the height and the vertical translation (under solenoid driving) of the moving part of the microactuators varies with time (in 
days) or after vibrating due to the contacting support of the trapezoidal plate lower corners or/and anchoring springs to the substrate, i.e., the smoothness of contacting changes with time and vibration when driven by solenoids. This is more pronounced with design 2 since its stiffness is lower than that of design 1 . The effect of the contact points is under investigation.

The following steps are used to bond design 2 using the method and bonding mechanism developed in this chapter.

Step_1). The microactuator of design 2 is vibrated driven by a solenoid (1 Hz) with an amplitude of $\sim \pm 100 \mu \mathrm{m}$ in both upward and downward directions for one hour to smooth the contact points between the lower corners of the plate (plus some points of the anchoring springs) and the substrate.

Step_2). A mirror plate is dropped on the central ring which is $\sim 1 \mathrm{~mm}$ above the ring. Heights of points along the line $\mathrm{AB}$ (Figure 3.12) are measured with the trench surface under the ring as the reference.

Step_3). The mirror plate is bonded to the microactuator following the bonding method using the bonding mechanism described in sections 3.1 and 3.2.

Step_4). The microactuator with bonding is measured again along the line AB. The measured results are compared with the measurements before bonding as shown in Figure 3.13 .

Measurements of the moving part of the microactutor are conducted before and after dropping the mirror plate on the ring to ensure no plastic deformation is caused by the mirror plate dropping.

Many small areas are scanned by high magnification lens to construct cross section profile along line $\mathrm{AB}$ (shown in Figure 3.12). Figure 3.13 shows the profile along $A B$ does not change before and after bonding. The micormirror developed in Chapter 2 is bonded using the bonding technology presented in this chapter which achieves a surface quality of $15 \mathrm{~m}$ in radius of curvature and $2 \mathrm{~nm}$ roughness. 


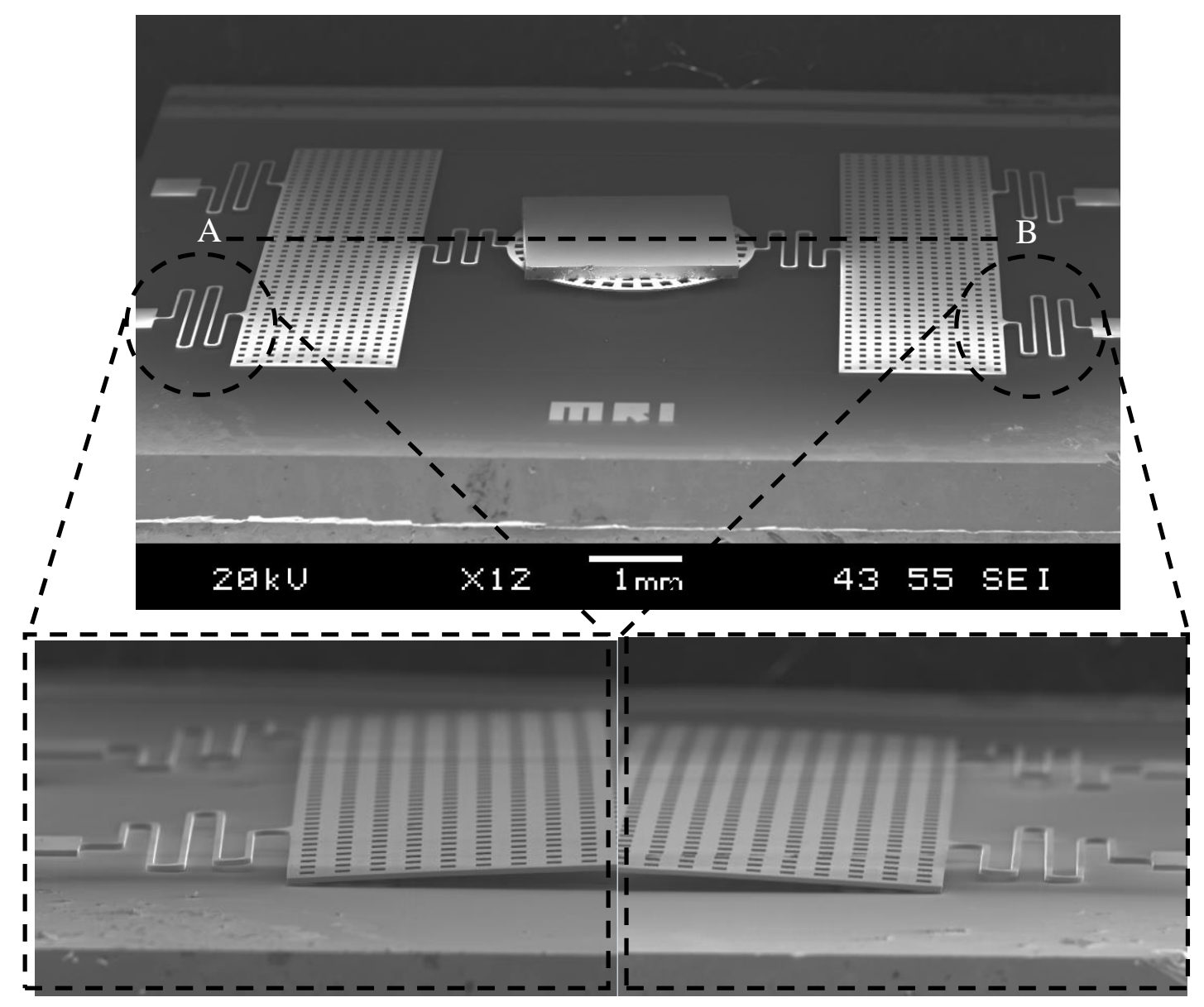

Figure 3.12 Mirror plate bonded on design 2

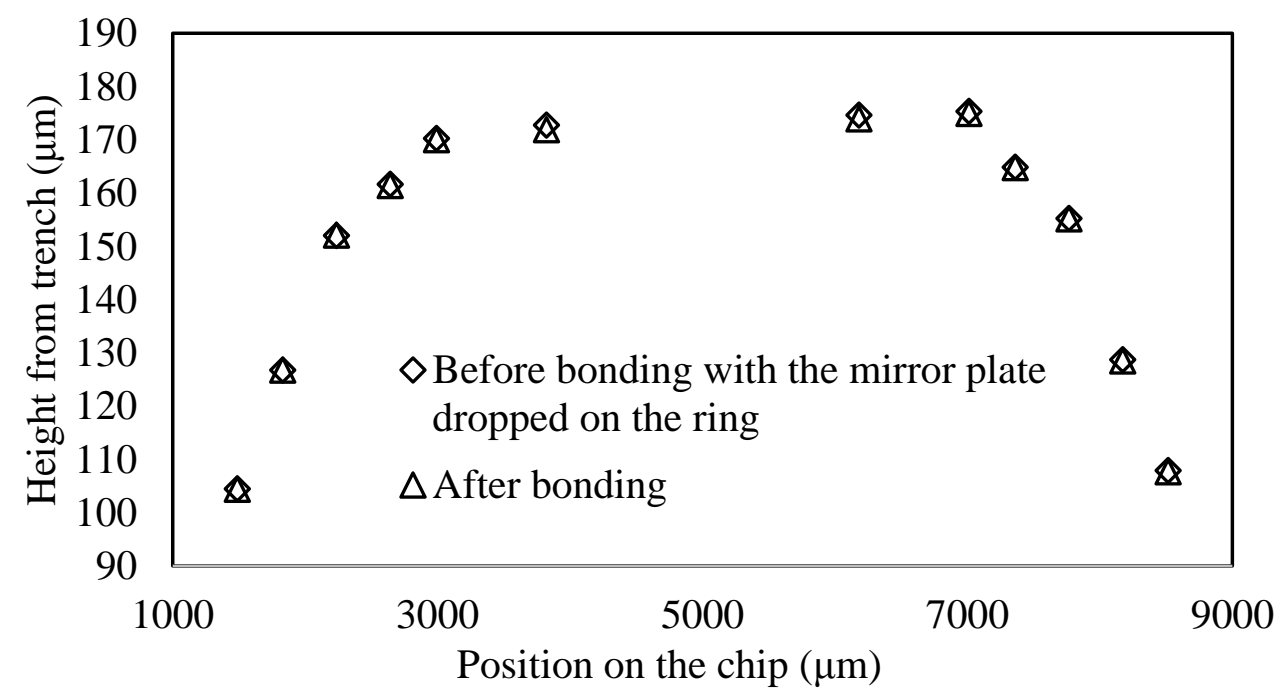

Figure 3.13 Cross section profiles (lines AB in Figure 3.12) of design 2 before and after bonding 


\subsection{Bonding characterization}

\subsubsection{Temperature test}

Two different types of high-temperature tests have been used to demonstrate that the bonding can work under high temperature. A laser test, as shown in Figure 3.15a, is used to verify the micromirror can reflect the high power laser. In this test, the power of the laser beam is continuously increased from a power adjustable laser transmitter, which increases the temperature continuously on the mirror plate, as shown in Figure 3.14. The bonding layer should have the same temperature as the mirror plate since enough time ( 5 minutes) is given to let the mirror plate surface temperature stabilize for each laser power, A cooled infrared camera (DirectIndustry FLIR SR5000) is used to measure the temperature. The laser power increases from $0.4 \mathrm{w}$ to $1.4 \mathrm{w}$ and the temperature is raised from $42.55{ }^{\circ} \mathrm{C}$ to $96.56{ }^{\circ} \mathrm{C}$. The tests stop at approximate $100{ }^{\circ} \mathrm{C}$. During the laser temperature experiment, the thickness of the bonding layer cannot be measured because the setup cannot be put under the 3D optical profiler. The experiment can only show the bonding survives temperature of $100{ }^{\circ} \mathrm{C}$.

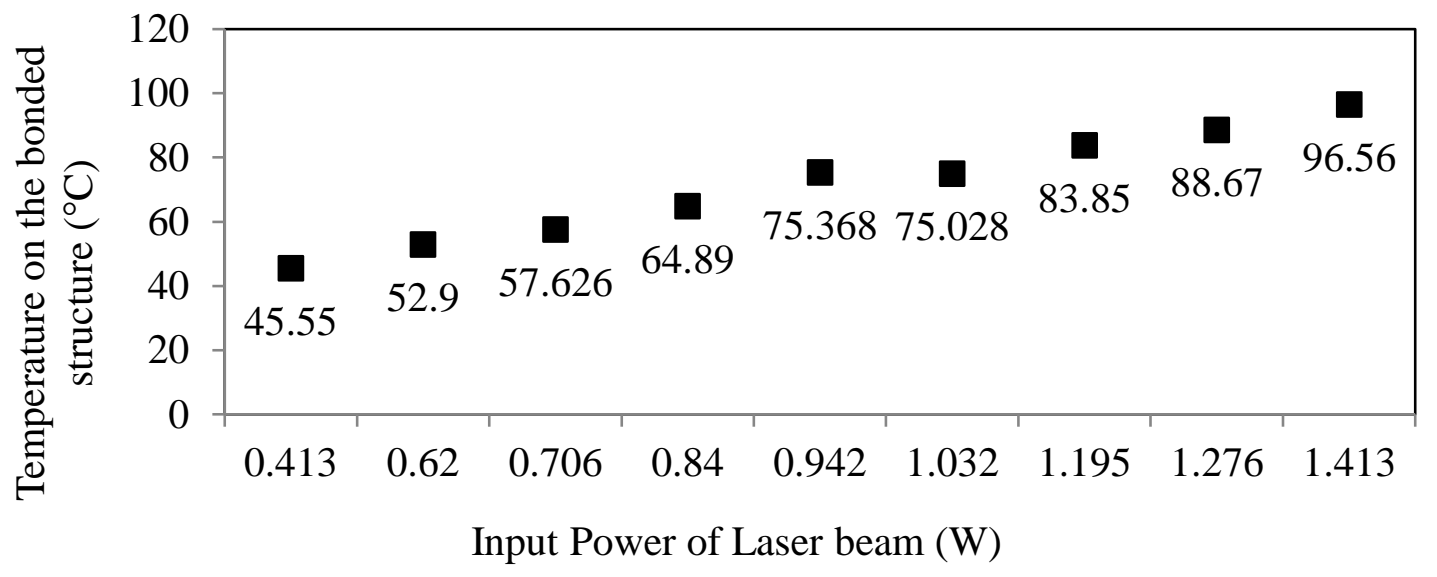

Figure 3.14 Temperature raised by increasing the power of the laser beam

The following test is able to measure the bonding layer at high temperature. As shown in Figure 3.15b, a heating chip is used to generate a high-temperature environment and the temperature on the bonded structure is monitored by a temperature detector which is glued on the backside of the chip using the thermal glue (EPO-TEK 353ND) [152]. The structure is kept in the high temperature for a few hours and heated to $80^{\circ} \mathrm{C}$, then the thickness of the bonding layer is measured using the 3D optical profile. The distances 
between multiple locations on the nickel film and the mirror surface do not change, which means the adhesive between them does not deform. This test verifies the micromirror and the bonding can work under temperature as high as $80{ }^{\circ} \mathrm{C}$.

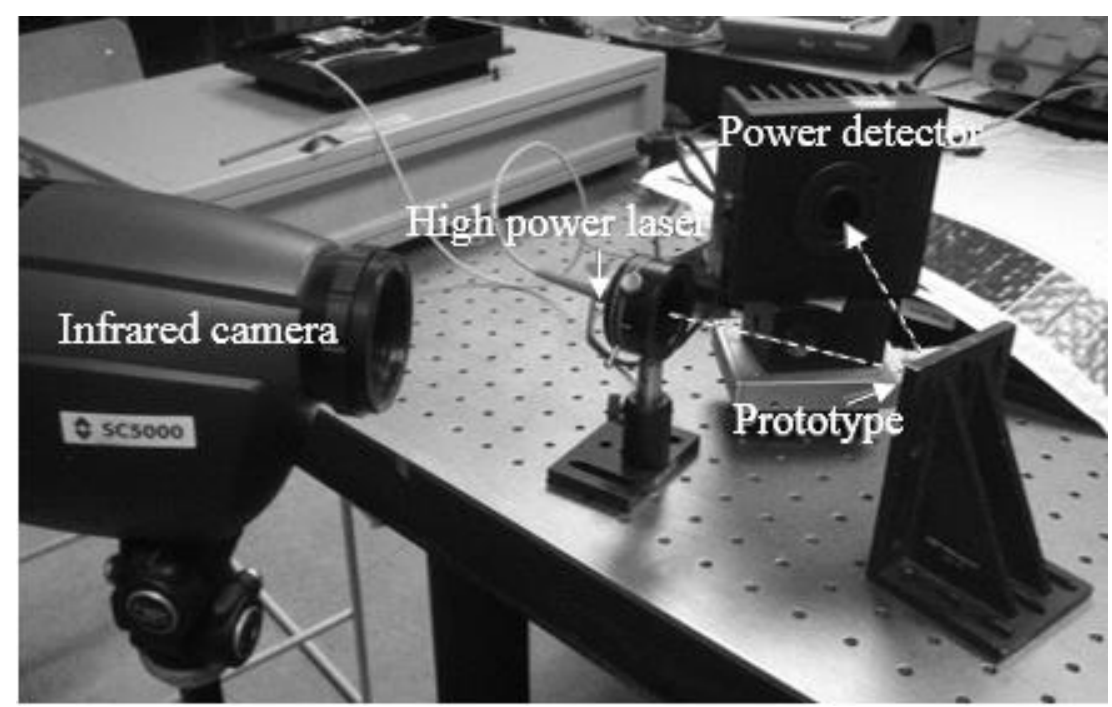

(a)

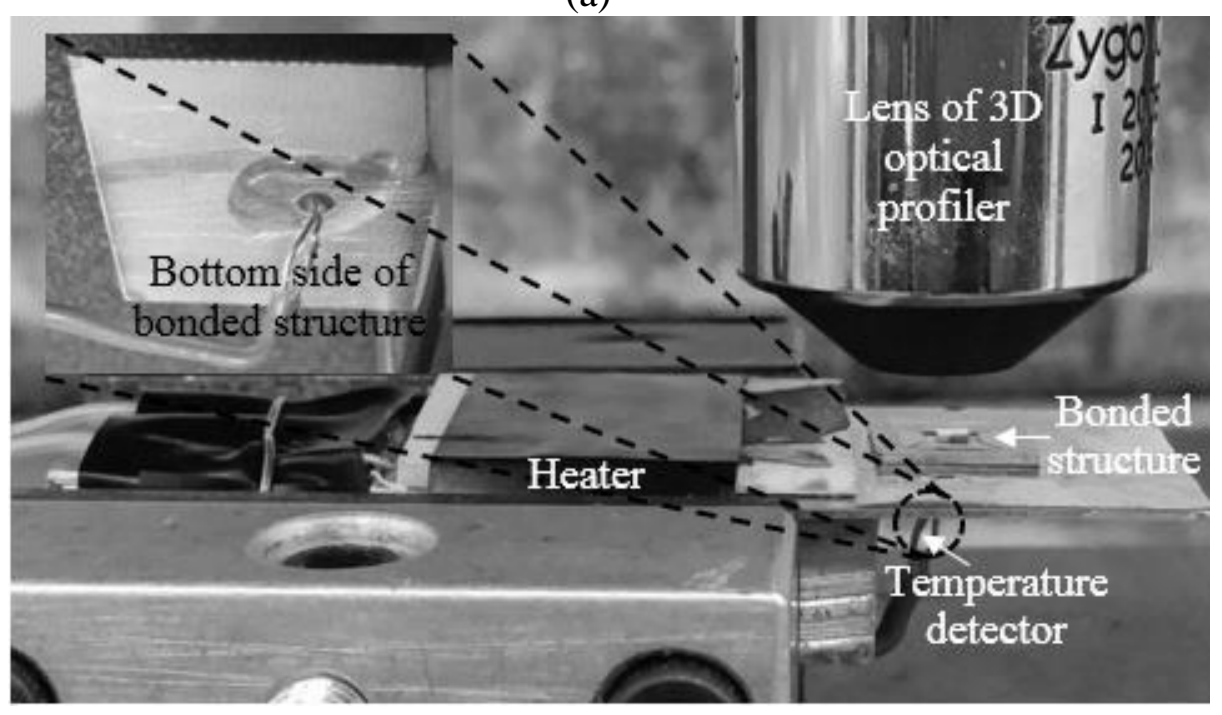

(b)

Figure 3.15 Test platforms. (a) High power laser test; (b) Heating test

For the low-temperature test, the bonded structure is placed in a freezer $\left(-30^{\circ} \mathrm{C}\right)$ for one hour and then moved to the room temperature to measure the thickness of the bonding layer, i.e., the distance between the nickel film and the mirror surface, which is not changed. In this section, the no change in bonding layer thickness is based on that the bonding layer thickness change is measured to be within $\pm 0.3 \mu \mathrm{m}$, which is the variation 
for multiple measurements since each height measurement has to made a reference plane as the starting point on the 3D optical profiler, i.e., remove the fringing lines as much as possible based on the user's eyes.

\subsubsection{Strength \& impact test}

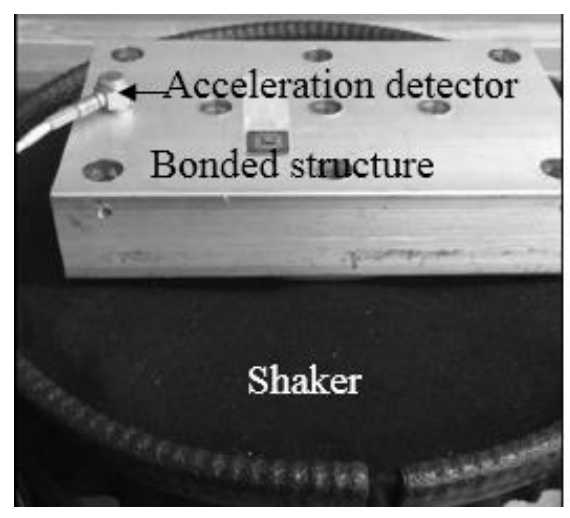

(a)

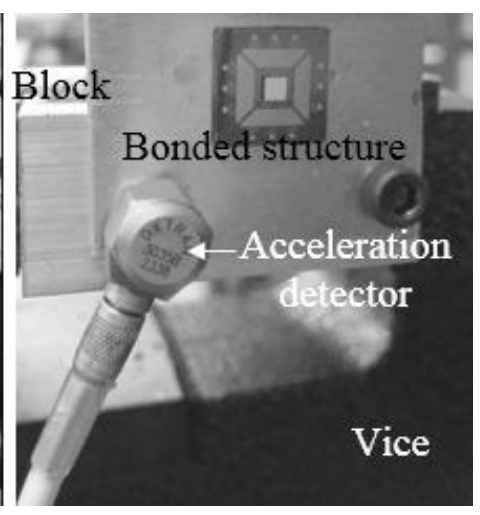

(b)

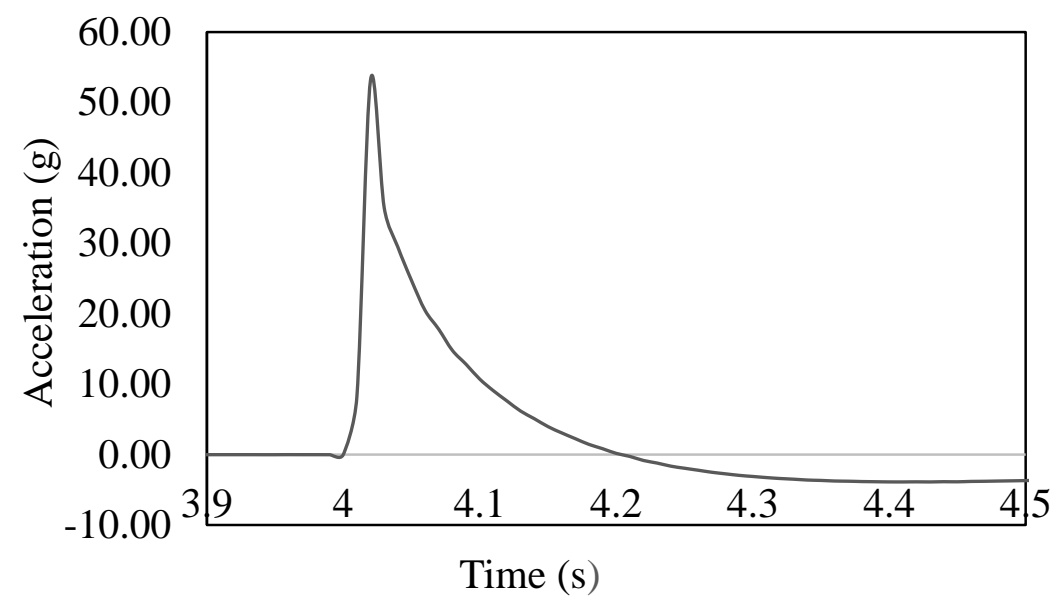

(c)

Figure 3.16 (a) Setup for strength test using the shaker; (b) Setup for impact test; (c) Result of impact test

In order to test the strength of the bonded structure, a shaker is employed to generate various frequencies $(20 \mathrm{~Hz}-200 \mathrm{~Hz})$ with difference accelerations $(2 \mathrm{~g}-5 \mathrm{~g})$ for several hours as shown in Figure 3.16a. There is no change in the bonding layer's thickness before and after the shaking test. That means the bonding layer does not become delaminated or loose. 
Considering the impact test may damage the moving film, it is performed at last. Since the bonded structure is already glued with a piece of aluminum sheet in the temperature test, the aluminum sheet is screwed on an aluminum block which is fixed by a vice for the impact test, as shown in Figure 3.16b. The acceleration is measured by an acceleration detector after the hammer pounds on the other side of the block. The peak acceleration is around $50 \mathrm{~g}$, as shown in Figure 3.16c. By multiplying with the weight of mirror plate $3.3 \mathrm{mg}$, the force on the adhesive can be calculated to be $1.6 \mathrm{mN}$. After the impact, the bonding layer thickness is measured to be unchanged.

\subsubsection{Performance of the bonded micromirror}

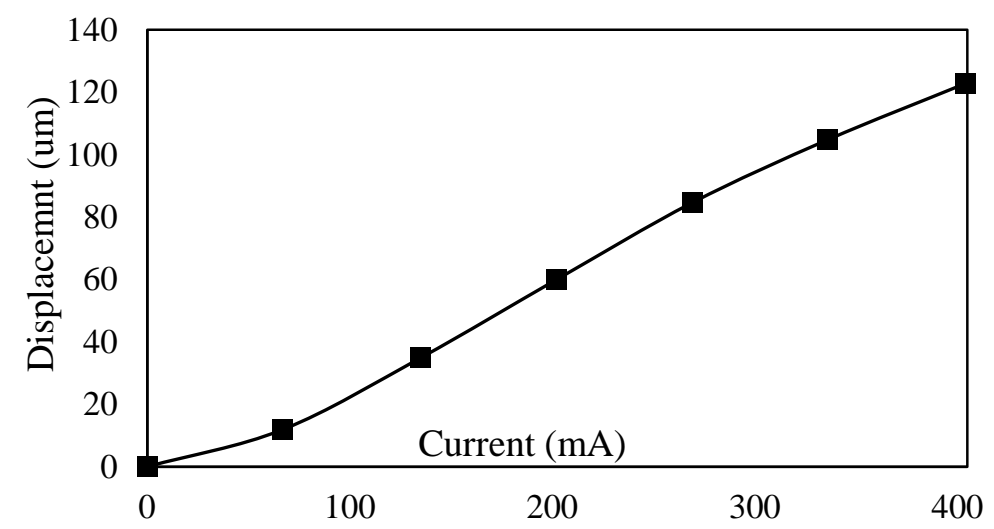

(a)

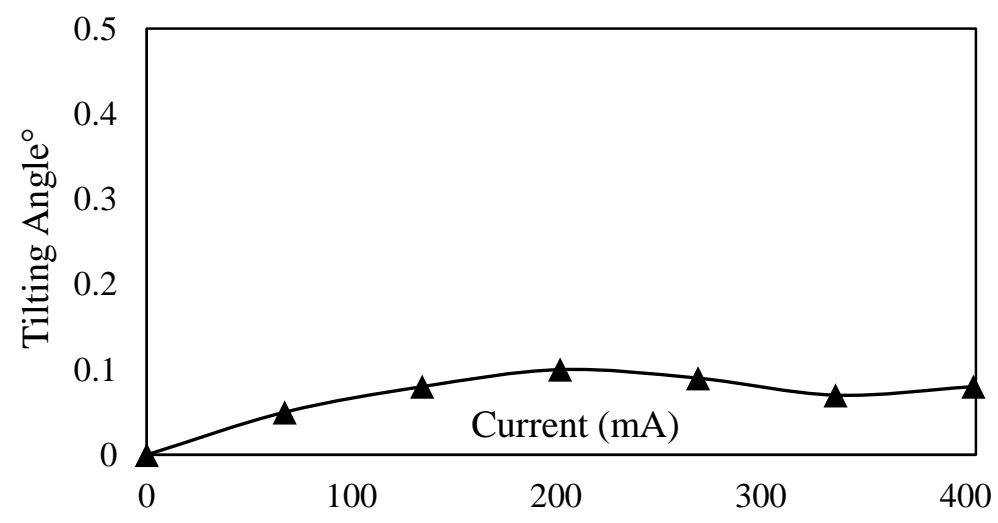

(b)

Figure 3.17 Displacement and tilting angle of the micromirror under various currents. (a) Displacement of micromirror; (b) Tilting angle of micromirror 
After the mirror plate is successfully bonded with the actuator, the static performance of the micromirror in design 1 is measured using the Zygo 3D optical profiler as shown in Figure 3.17a. The maximum displacement is $120 \mu \mathrm{m}$ which is measured at $400 \mathrm{~mA}$, which is similar to that in [25] but not exactly the same due to two different prototypes. Tilt during the micromirror movement is around $0.1^{\circ}$, as shown in Figure $3.17 \mathrm{~b}$.

\subsection{Summary}

A novel bonding technology is presented in this chapter, which enables bonding of a high surface quality mirror plate to any released microactuator containing the ferromagnetic layer, to achieve a large displacement and high surface quality. The novel bonding technology uses the magnetic field pulling-force to bring the moving part of the released actuator in contact with a mirror plate. Plastic deformation or damage to the released microactuator is avoided and the risk of sticking the actuator moving part to the substrate due to the squeezed out adhesive is eliminated. Prototypes of two designs are fabricated and successfully bonded using the bonding process proposed in this chapter, which experimentally verifies the bonding method. Experiments were conducted to characterize the bonding layer, i.e, withstanding vibration in frequency $20 \mathrm{~Hz}-200 \mathrm{~Hz}$ with $2 \mathrm{~g}-5 \mathrm{~g}$ acceleration, surviving temperature range of $-30{ }^{\circ} \mathrm{C} \sim 80{ }^{\circ} \mathrm{C}$. The bonded translation micromirror achieved $15.6 \mathrm{~m}$ curvature radius and $2 \mathrm{~nm}$ roughness with 120 $\mu \mathrm{m}$ quasi-static displacement. 


\section{CHAPTER 4 A REPULSIVE MAGNETIC FORCE DRIVEN TRANSLATION MICROMIRROR}

This chapter is based on the following paper:

Y. Xue, H. Zuo and S. He, "A repulsive magnetic force driven translation micromirror," J. Micromechanics Microengineering, in-press.

This chapter presents a repulsive magnetic force driven micromirror with large displacement and high surface quality, which meets the third objective of this thesis. The micromirror well solves the limitation of the previous design, i.e., large variation in translation starting position and low repeatability, caused by the touching points between the moving film and substrate before and in operation. The new design utilizes a novel driving mechanism, i.e., permanent magnet ring above and electromagnet underneath the moving film, to lift the moving film from touching the substrate and generate repulsive magnetic force (instead of attractive force in the previous design) to push the moving film up and away from the substrate for translation. Due to the touching, the previous design has to pre-oscillate for 20-30 minutes at $1 \mathrm{~Hz}$ before usage (after resting for a few hours) to reduce the starting position variation from $\sim 15 \mu \mathrm{m}$ to $3 \sim 4 \mu \mathrm{m}$. Even after the preoscillation, the repeatability is still low, which is $14.2 \%$ because of the touching in operation. In the novel design presented in this chapter, the touching between the moving film and the substrate is completely eliminated before and in operation. As a result, the starting position of the translating mirror is constant each time without any pre-oscillation and the repeatability is $<1 \%$. In addition, even the residual stress gradient based clamped-clamped microactuator is time stable according to our tests in past few years, it is reported in [21] that the residual stress is not stable through thermal cycles, while this design does not need the residual stress gradient to curve up the moving film. The maximum displacement of $144 \mu \mathrm{m}$ can be achieved when $140 \mathrm{~mA}$ current is applied on the electromagnet. As an application, the micromirror is used as the movable mirror in a Michelson interferometer to measure the wavelength of a laser beam. The result shows a measurement error of $2.19 \%$ for a $532 \mathrm{~nm}$ laser beam. 


\subsection{Micromirror design}

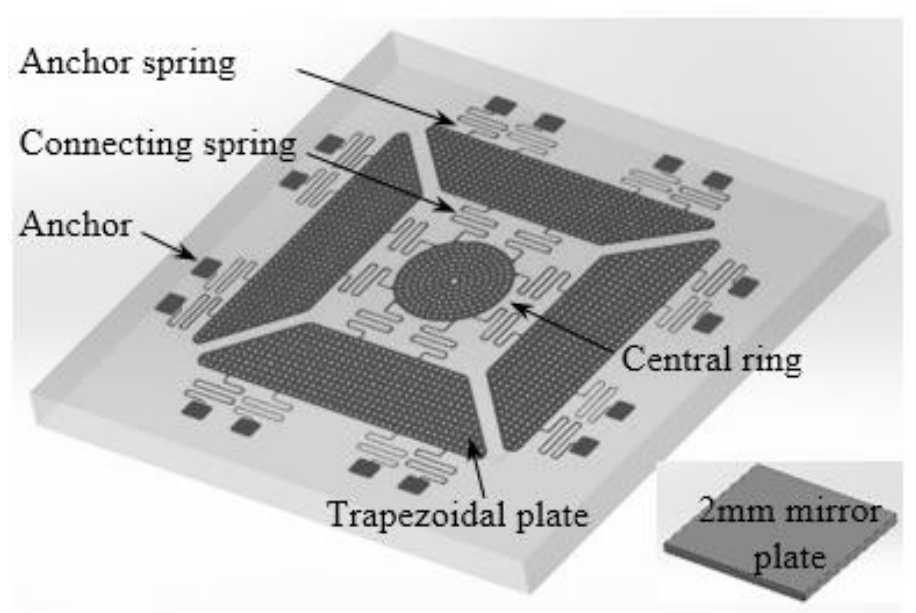

(a)

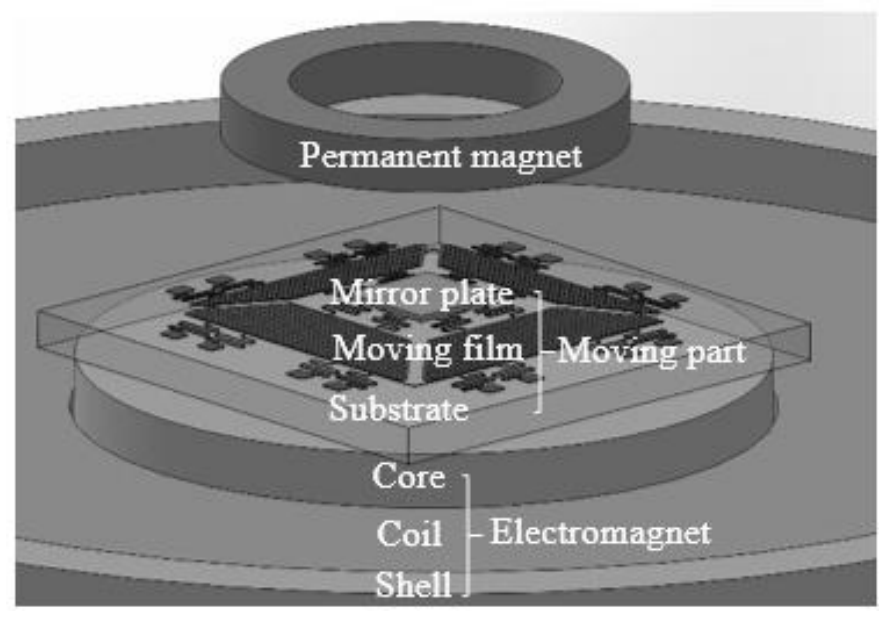

(b)

Figure 4.1 Concept of the micromirror. (a) Moving film of the micromirror; (b) The fixed and moving part of the micromirror

The micromirror includes a moving part and a fixed part. The moving part contains the moving film fabricated by MetalMUMPs [139] and a mirror plate fabricated by dicing a polished silicon wafer with a thin metal coating layer, as shown in Figure 4.1a. These two parts are fabricated separately and bonded together by a non-touching bonding technology developed in [147]. The fixed part includes a ring shape permanent magnet above the moving part and an electromagnet under it, as shown in Figure 4.1b. After release, the moving film curves up due to the residual stress gradient in the nickel thin 
film. The lower corners of each trapezoidal plate touch the substrate before assembling the permanent magnet, as shown in Figure 4.2. After assembling the permanent magnet ring above the moving film, the lower corners of trapezoidal plates are lifted up to separate from the substrate as shown in Figure 4.3a. After the current is applied to the electromagnet, the repulsive electromagnetic force is generated on the trapezoidal plates to push them to move upward and away from the substrate as shown in Figure 4.3b. Thus no contact occurs before and in operation such as to eliminate the problem of large variation in the micromirror starting position before the operation and low repeatability in operation caused by contacting points as in the previous design [90]. The magnetic lines in Figure 4.3 indicate the direction of the magnetic field with its density not to scale of the magnetic field strength.

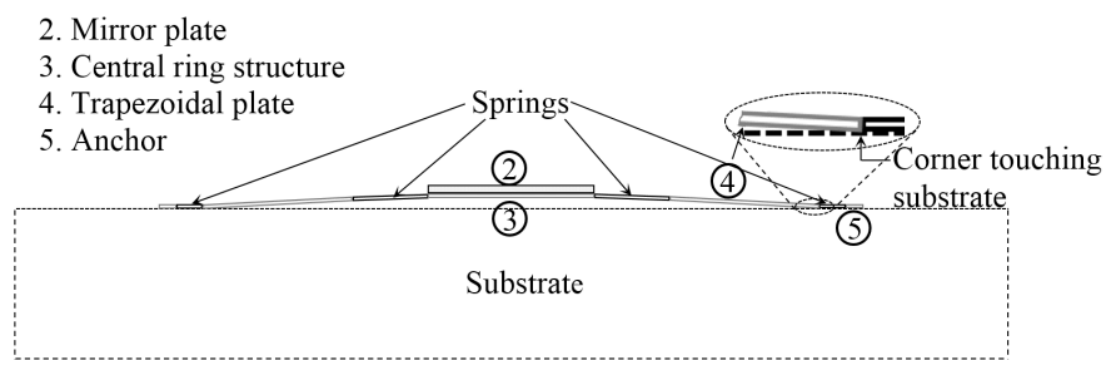

Figure 4.2 Lower corners of each trapezoidal plate touching on the substrate before the permanent magnet is placed

The following explains how the repulsive magnetic force is generated. Since the surface dimension of each trapezoidal plate is much greater than the thickness (millimeters vs $20 \mu \mathrm{m}$ ), the high shape anisotropy keeps the magnetization in-plane when an external magnetic field is applied. For example, if the permanent magnet ring has the magnetic north pole on the top surface and the south pole on the bottom, without applying current to the electromagnet the north and south poles are formed on the upper and lower edges of each trapezoidal plate respectively, as shown in Figure 4.3a. In order to move the moving film upward, the magnetic poles on the trapezoidal plate upper edge and the top surface of electromagnet core need to be the same, which makes them repel each other. To achieve this purpose, two conditions need to be met: 1) Counterclockwise (in this prototype) current has to be applied to the electromagnet to form a magnetic north pole on the top surface of the core, as shown in Figure 4.3b; and 2) Dimension of the core needs to be larger than the moving film to ensure that the magnetization is directed 
towards the upper edge from the lower edge of each trapezoidal plate. Thus the upper edge of each trapezoidal film is the north pole; otherwise, the magnetization is opposite [140] and the moving film moves downward as explained in Appendix B. In this example, the dimension of the moving film is $8 \mathrm{~mm} \times 8 \mathrm{~mm}$ excluding anchor and the diameter of the electromagnet core is $10 \mathrm{~mm}$. The magnetization in the nickel film needs to be controlled below the saturation level.

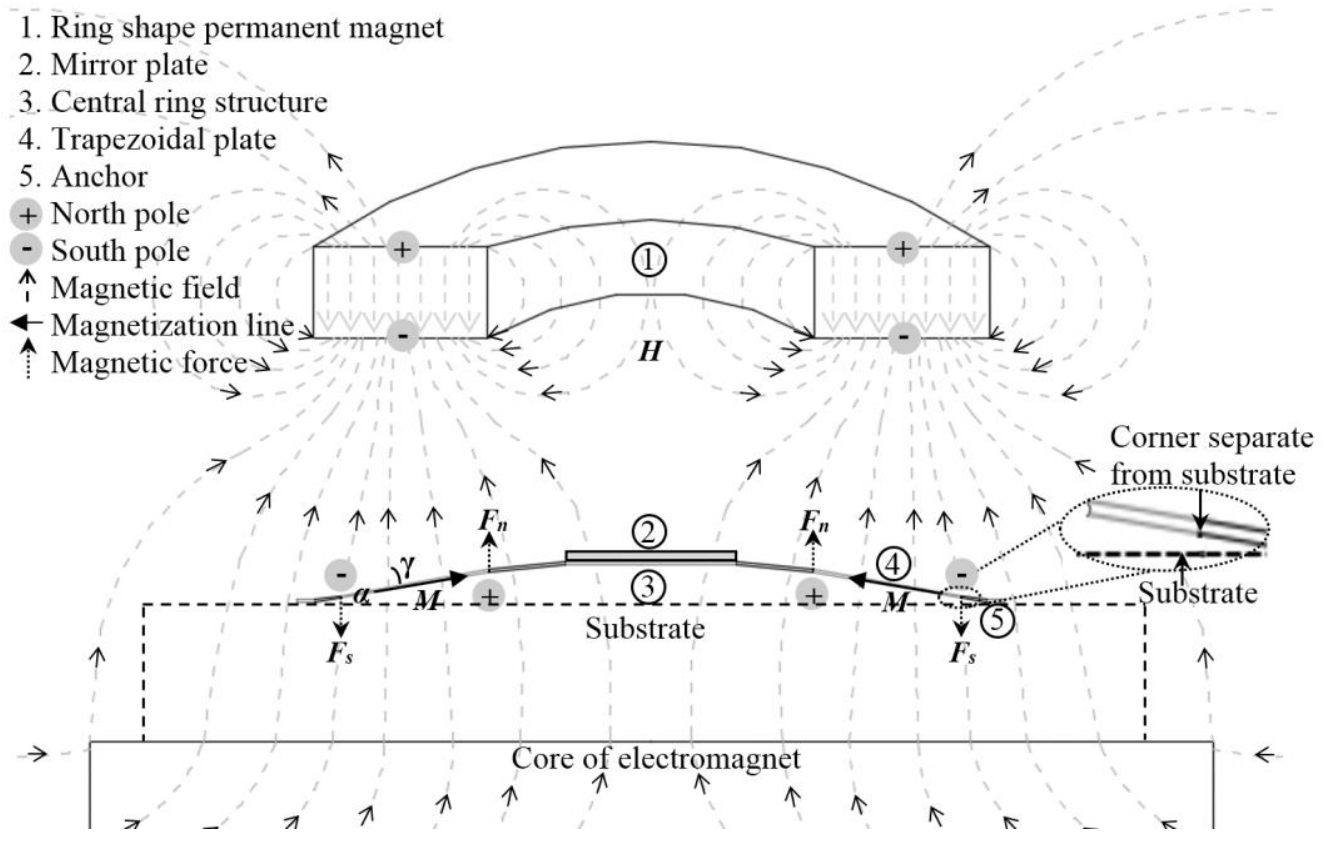

(a)

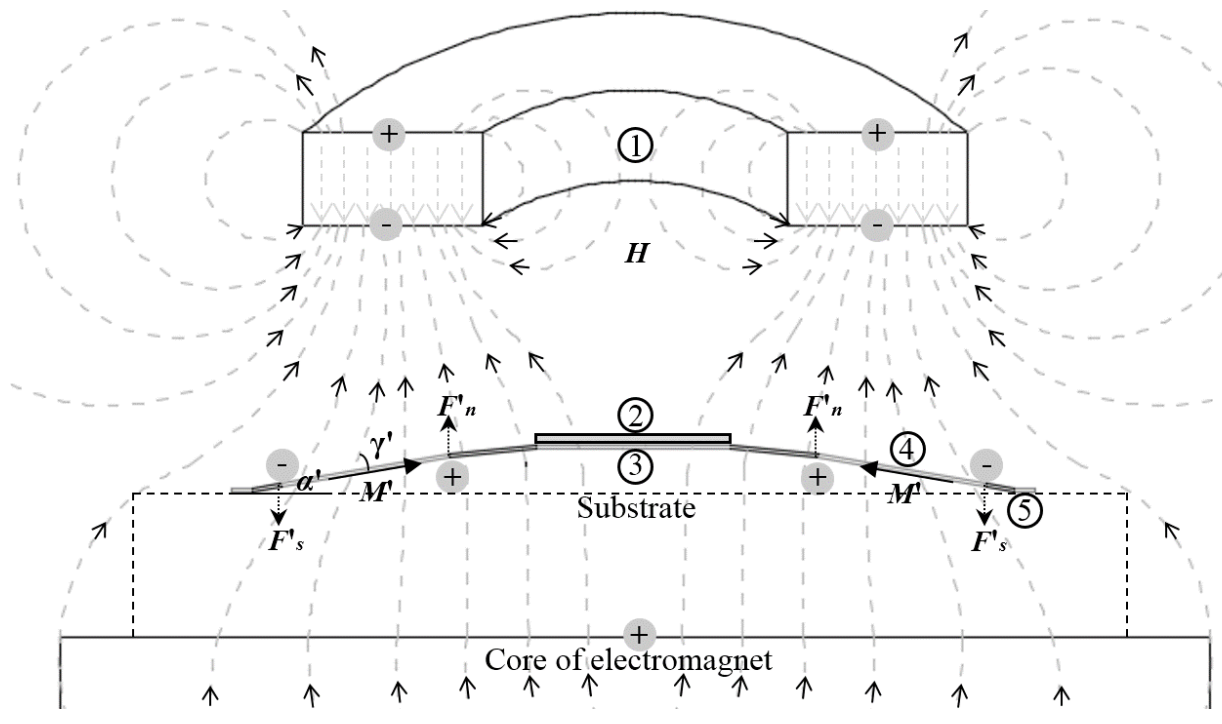

(b)

Figure 4.3 Working princinple of mivromirror. (a) Moving part pre-lifted by the permanent magnet; (b) Moving part moves upward (current applied) 
On the other hand, the magnitude of the magnetization in the designed nickel film is about $0.4-0.5 \mathrm{~T}$ at $140 \mathrm{~mA}$ current, which is less than the saturation magnetization of the nickel-iron film ranging from $1.1 \mathrm{~T}(\mathrm{Ni80 \% Fe} 20 \%)$ to $0.7 \mathrm{~T}$ (Ni95\%Fe5\%) [140]. So by controlling the driving current, the generated magnetic field can be controlled and then the in-plate magnetization level in each trapezoidal plate can be controlled. Therefore the translation of the central ring structure along with the mirror plate can be controlled as well, i.e., the higher current to the electromagnet, the larger upward translation of the mirror plate. In addition, the deformation of the magnetized central ring structure can be neglected, because the total magnetic force generated on it is zero.

\subsection{FEM simulation}

FEM software of Ansys $®$ Multiphysics and CoventorWare $®$ is used to simulate the displacement of the micromirror. The dimensions of the micromirror are shown in Table 4.1. The deformation of the moving film caused by residual stress gradient, permanent magnet and electromagnet are simulated in this section. The residual stress gradient introduced initial curve-up is not necessary since this design does not rely on the residual stress gradient to curve up the moving film. It uses a permanent magnet ring above the moving film to lift the moving film up. However, it needs to be considered and simulated since MetalMUMPS is used for prototyping which brings residual stress in the fabrication process. The residual stress gradient caused displacement of the moving film is $168 \mu \mathrm{m}$ as simulated in [90], which is the displacement of the central ring from its unreleased position.

The Ansys Magneto-Static module is used to simulate the magnetic force acting on the moving film generated by the permanent magnet and the electromagnet. Due to the symmetric structure, only a quarter of the micromirror is modeled in the simulation, as shown in Figure 4.4. Iterations are used to obtain the magnetic force on each element of the moving film and the corresponding deformation as follows. In the 1st iteration, the magnetic forces $\left(\mathrm{F}_{1 \mathrm{st}}\right)$ is obtained in Ansys based on the original deformation (Def $\left.f_{\text {original }}\right)$ caused by the residual stress gradient in the absence of any magnetic force, then the deformation $\left(\operatorname{Def}_{1 \mathrm{st}}\right)$ is obtained from ConventorWare by applying the residual stress and the magnetic force $\left(\mathrm{F}_{1 \mathrm{st}}\right)$ on the model of Def $\mathrm{forinal}_{\text {. In }}$ the 2 nd iteration, a new deformation $\left(\right.$ Def $\left._{2 n d}\right)$ is obtained by applying the new magnetic force $\left(F_{2 n d}\right)$ obtained 
based on the model of deformation $\left(\mathrm{Def}_{1 \mathrm{st}}\right)$. The iteration continues until the deformation is stabilized when the variation is about $2 \%$. The simulated displacements at various currents are shown in Figure 4.9 in section 4.3.2.2.

Table 4.1 Dimensions of the micromirror

\begin{tabular}{|c|c|c|c|}
\hline Part name & Dimension & Part name & Dimension \\
\hline Mirror plate & $2 \mathrm{~mm} \times 2 \mathrm{~mm}$ & Moving film thickness & $20 \mu \mathrm{m}$ \\
\hline $\begin{array}{c}\text { Mirror plate } \\
\text { thickness }\end{array}$ & $120 \mu \mathrm{m}$ & Central ring & $2 \mathrm{~mm}$ (diameter) \\
\hline $\begin{array}{c}\text { Upper edge of } \\
\text { trapezoidal plate }\end{array}$ & $3 \mathrm{~mm}$ & Connection spring & $0.9 \mathrm{~mm}(\mathrm{~L}) \times 0.8 \mathrm{~mm}(\mathrm{~W})$ \\
\hline $\begin{array}{c}\text { Lower edge of } \\
\text { trapezoidal plate }\end{array}$ & $6 \mathrm{~mm}$ & Anchor spring & $0.8 \mathrm{~mm}(\mathrm{~L}) \times 0.9 \mathrm{~mm}(\mathrm{~W})$ \\
\hline $\begin{array}{c}\text { Height of trapezoidal } \\
\text { plate }\end{array}$ & $1.5 \mathrm{~mm}$ & Electromagnet & $31.8 \mathrm{~mm}(\mathrm{D}) \times 12.7$ \\
$\mathrm{~mm}(\mathrm{H})$
\end{tabular}

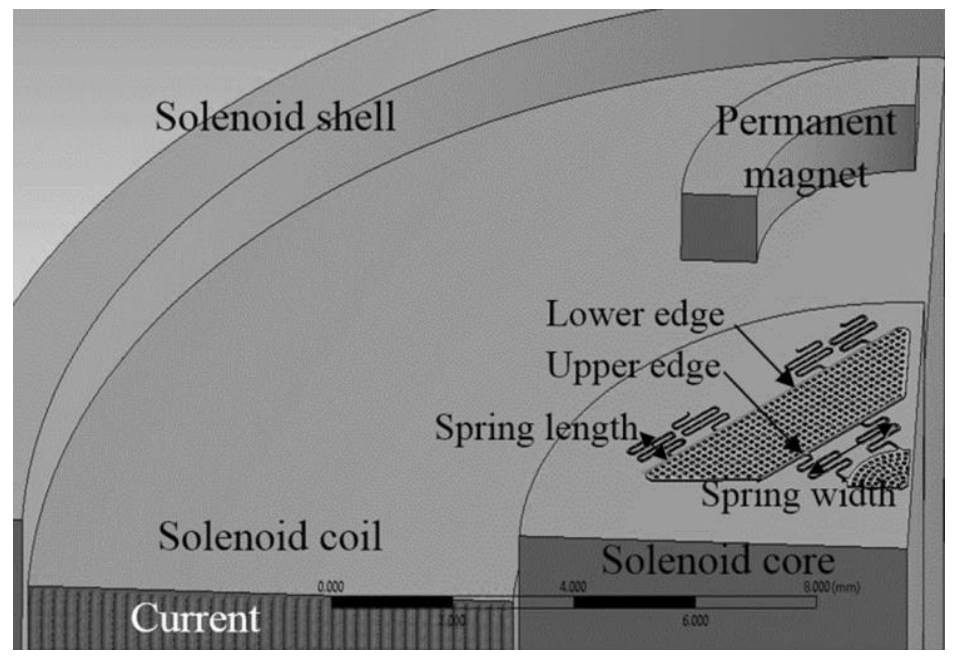

Figure $4.43-\mathrm{D}$ model of a quarter of the micromirror

In order to verify that the maximum stress in the micromirror's moving part is less than the yield strength, the moving part is simulated using CoventorWare. The simulation result 
shows a translation of $710 \mu \mathrm{m}$ can be achieved with the maximum stress smaller than the yield strength which is $400 \mathrm{MPa}$ [94], as shown in Figure 4.5.

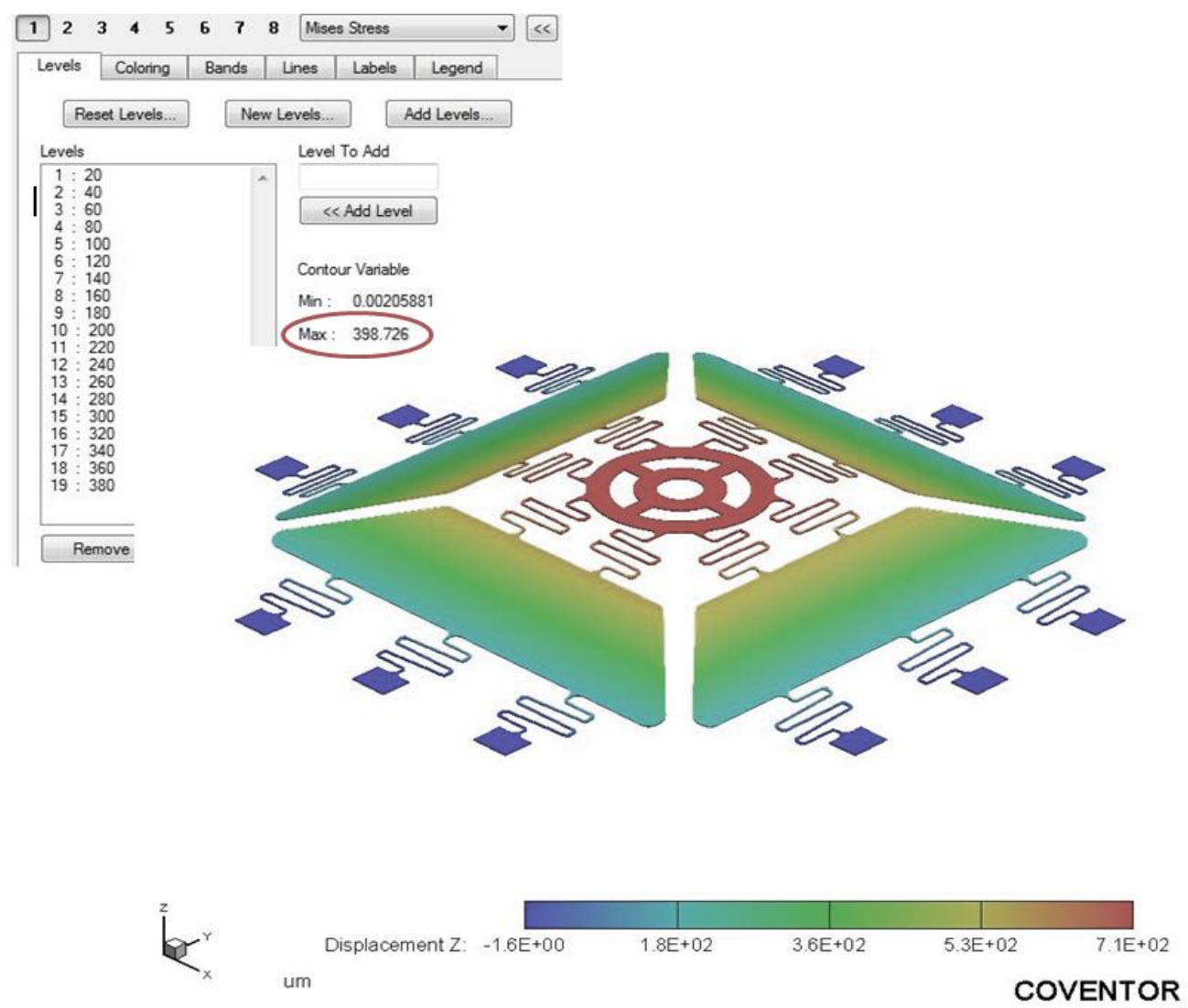

Figure 4.5 Stress simulation using CoventorWare

\subsection{Experiments}

\subsubsection{Prototype}

The chip of the moving part presented in this chapter is in a wire bonded package CQFP 120 as shown in Figure 4.6. The wire bonding is for the Lorentz-force experiments, which is not used in the chapter. Two parts are designed to assemble the moving part, the ring shape permanent magnet and the electromagnet (E-05-125-34 from Magnetic Sensor System) together as a micromirror as shown in Figure 4.6. The electromagnet holder has a $1 \mathrm{~mm}$ deep square hole opened on the top to hold the packaged moving part and a $4 \mathrm{~mm}$ deep circular hole on the other side to accommodate the electromagnet. The permanent magnet (PM) holder is designed to hold the ring shape permanent magnet $4 \mathrm{~mm}$ above the trench of the moving part which is limited by the 
working distance of the lens of the optical profiler. Four holes surrounding the permanent magnet, as shown in Figure 4.6b, as windows are used to observe the trapezoidal plate.

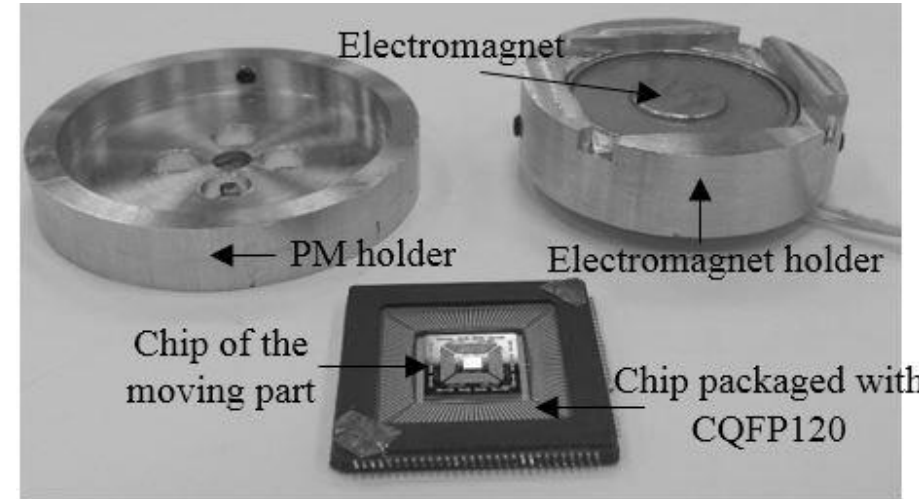

(a)

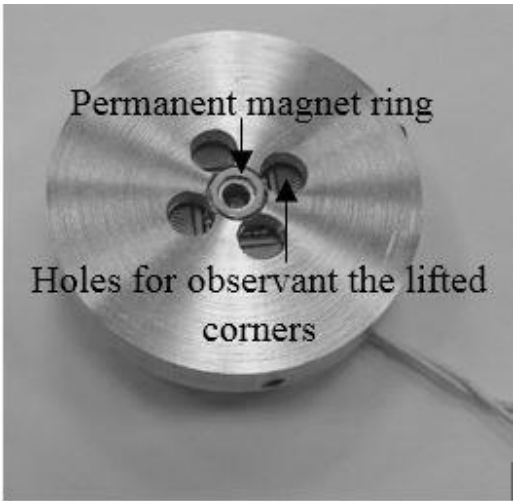

(b)

Figure 4.6 Micromirror before and after assembly. (a) Micromirror before the assembly;

(b) Assembled micromirror

\subsubsection{Performance}

The performance of the assembled micromirror is measured by a Zygo 3D profiler, including the initial curve-up of the moving part by the residual stress gradient, the prelift height by the permanent magnet and the upward translation when a current is applied to the electromagnet.

\subsubsection{Deformation/displacement of moving film before applying current}

The moving film curved up due to the residual stress gradient is observed using a SEM as shown in Figure 4.7a. The lower corners of each trapezoidal plate touch the substrate surface. Each trapezoidal plate is warped to form a convex shape as shown in Figure $4.7 \mathrm{~b}$ and explained in [90]. Figure 4.8a shows the contact since the distance between the substrate and the top surface of the corner is about $20 \mu \mathrm{m}$ which is almost the nominate thickness of the nickel layer of the MetalMUMPs process. The nominate thickness of nickel film in MetalMUMPs is $20 \mu \mathrm{m}$ which varies with different fabrication runs, as well as various locations on the same silicon wafer depending on the local structure. The average height of the initial curve-up of the moving film in this experiment is $160 \mu \mathrm{m}$ which is $4.7 \%$ different from the simulation. When the mirror plate is bonded on the central ring of the moving film using the bonding technology developed in [147], no plastic deformation or damage due to the bonding process is found. After the micromirror 
is assembled, the moving part pre-lifted by the permanent magnet ring is measured. Based on the measurement result, all the corners of the trapezoidal plates are separated from the substrate under the magnetic field generated by the ring shape permanent magnet, as shown in Figure 4.8a, which shows about $70 \mu \mathrm{m}$ lifted up from the substrate. A $120 \mu \mathrm{m}$ thick mirror plate is used in this design. Some scratches are observed on the mirror surface due to the flaw during fabrication. It has a radius of curvature of $9.2 \mathrm{~m}$ and surface roughness of $12 \mathrm{~nm}$.

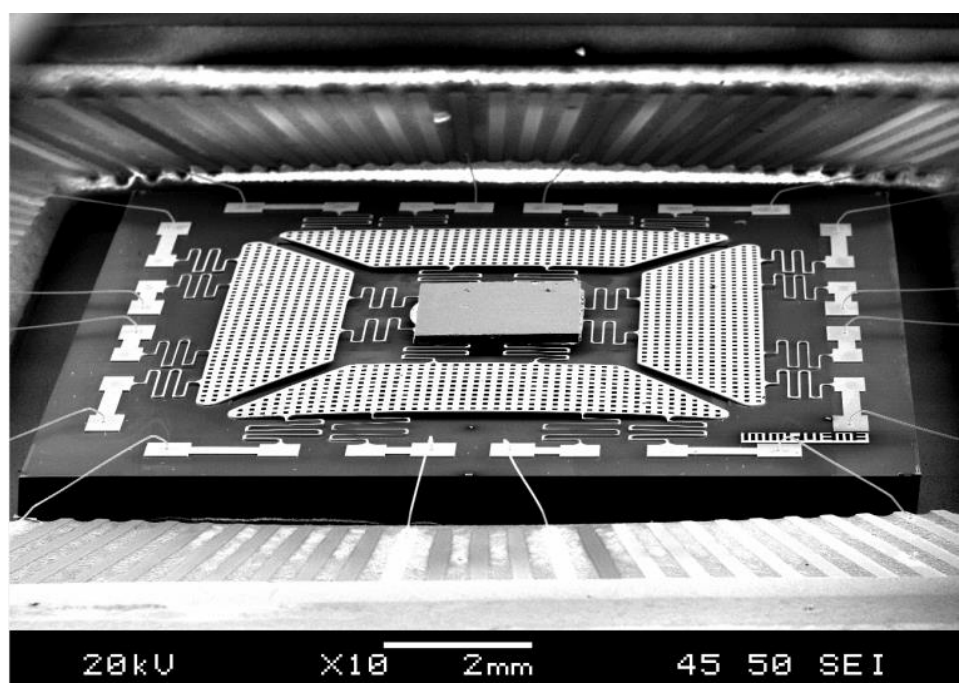

(a)

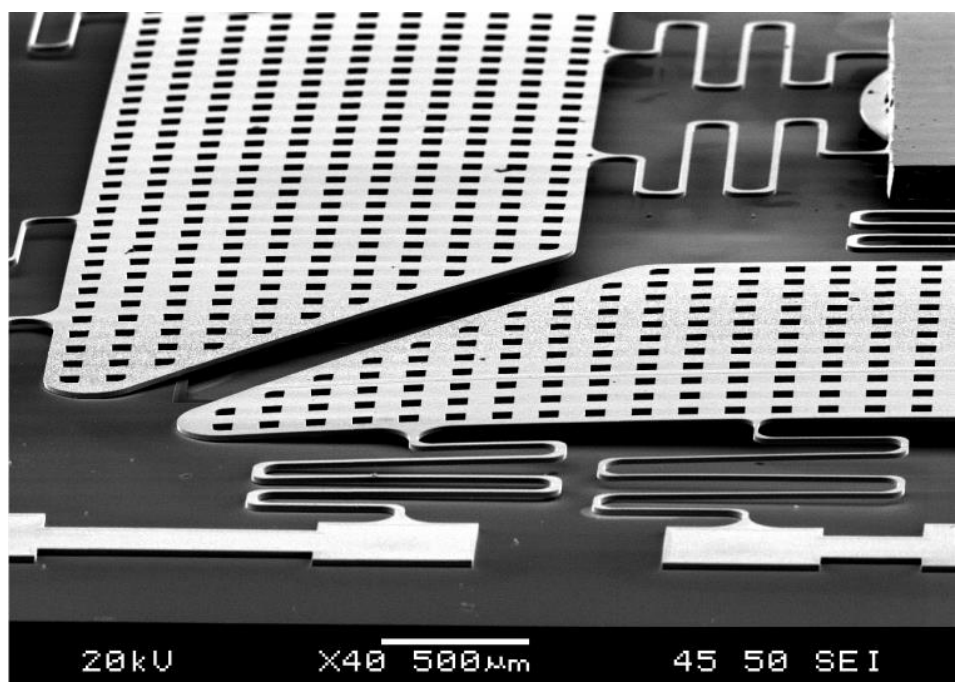

(b)

Figure 4.7 SEM images show the corners touching on the substrate before assembly. (a) Prototype of the moving part before assembly; (b) Trapezoid plate lower corners touching on the substrate 


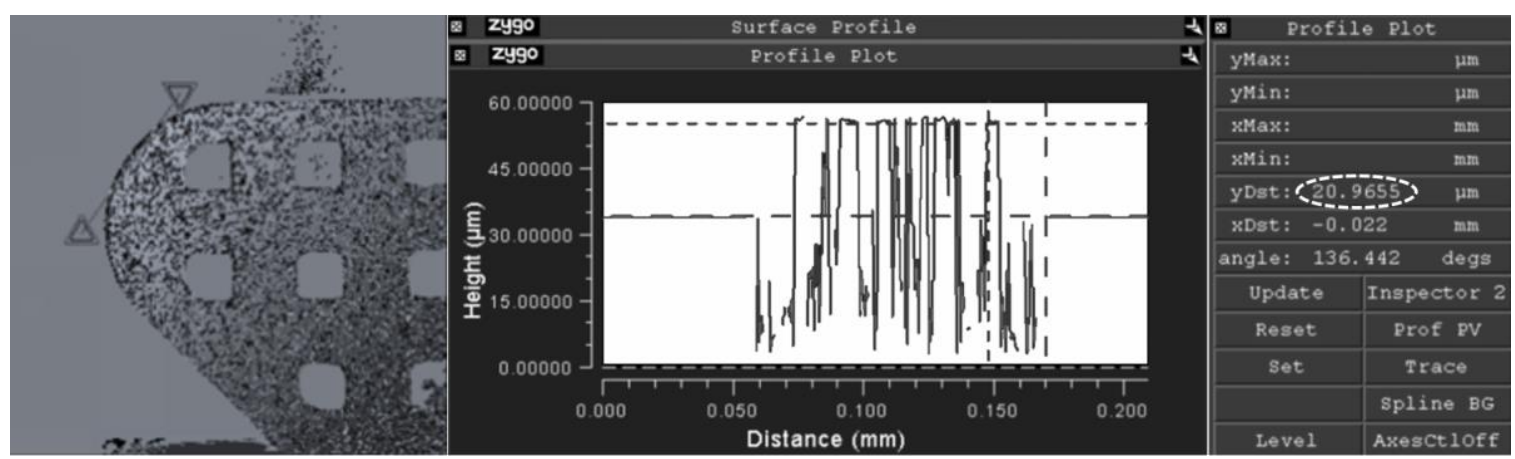

(a)

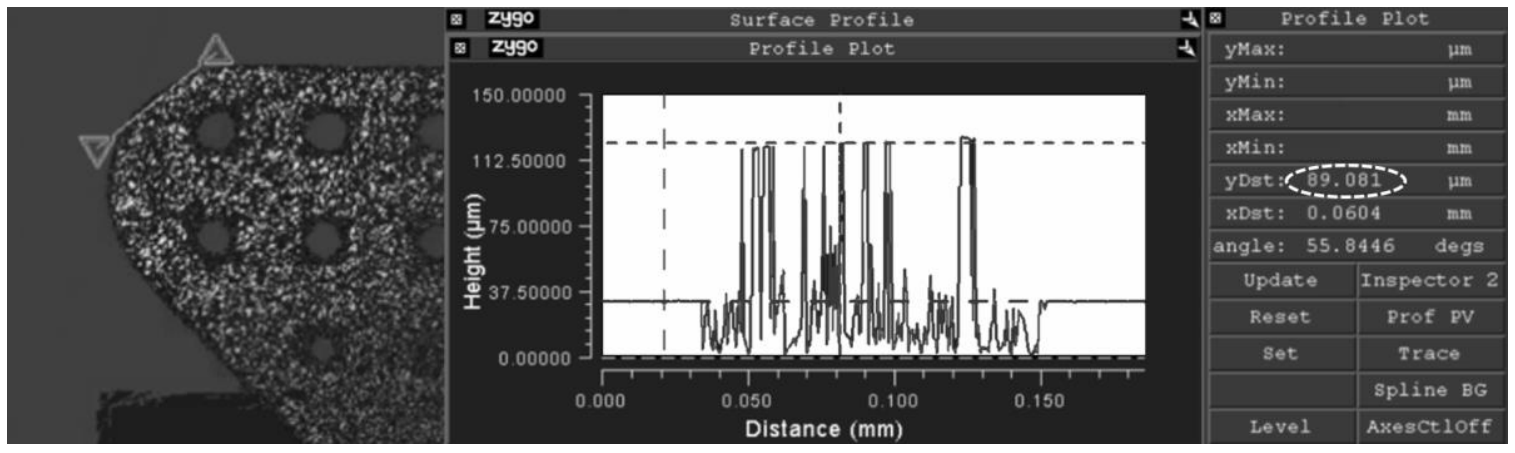

(b)
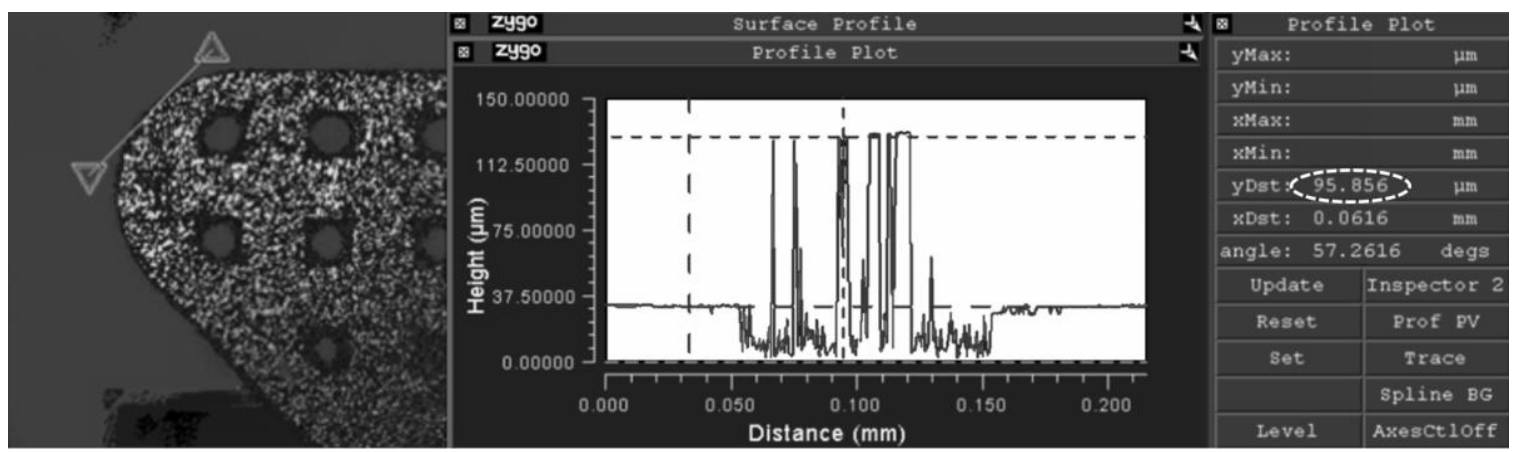

(c)

Figure 4.8 The lower corners of trapezoidal plate are separated from substrate in the permanent magnet and repulsive magnetic force translation micromirror. (a) The lower corner of trapezoidal plate touching on the substrate before the permanent magnet and the electromagnet assembled; (b) The lower corner of trapezoidal plate separated from the substrate after assembling the permanent magnet before applying current through the electromagnet; (c) The lower corner of trapezoidal plate moves upward after current is applied to the electromagnet (This figure shows the example of applying low current) 


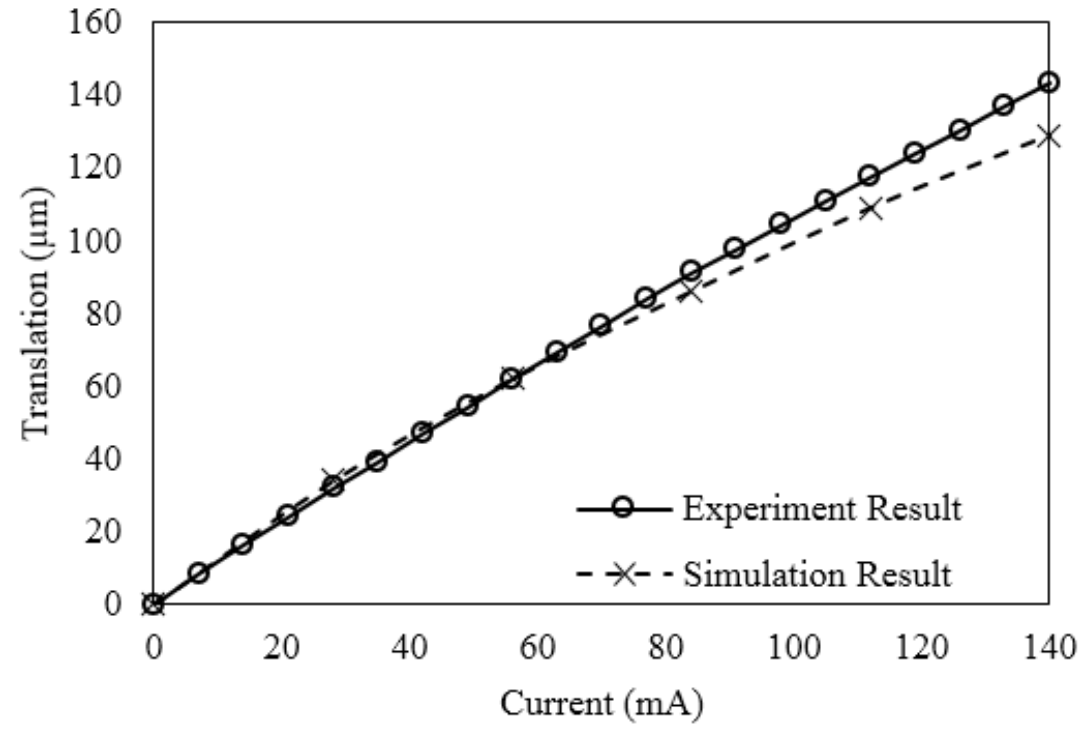

(a)

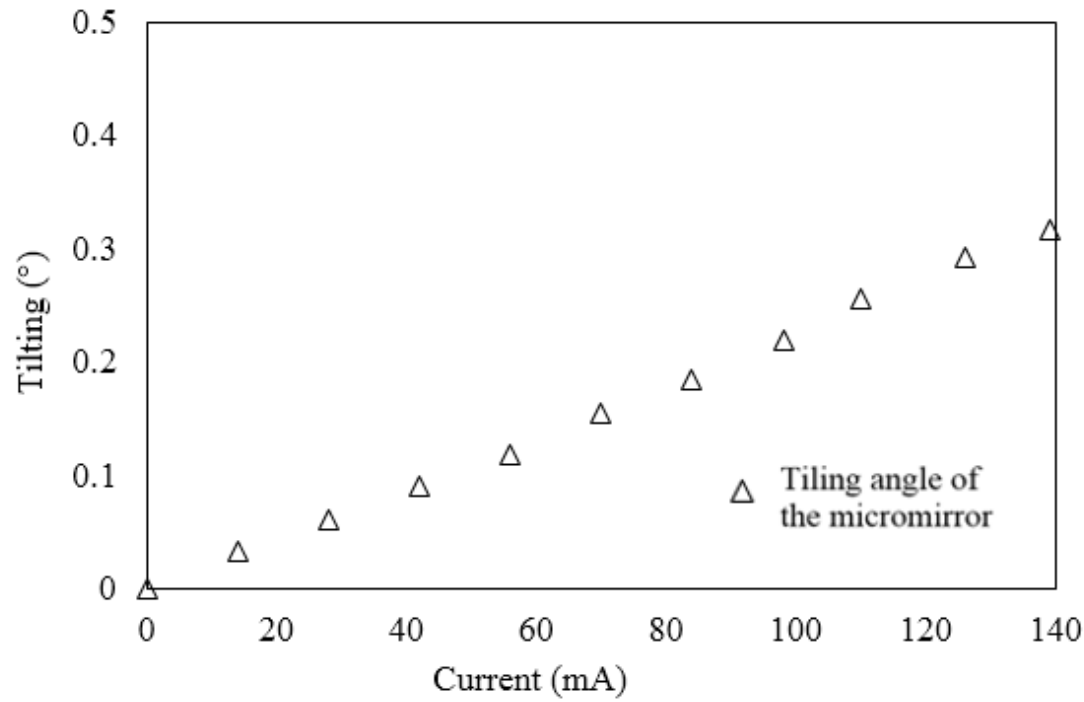

(b)

Figure 4.9 Micromirror translation in static model. (a) Translations in experiment and simulation; (b) Tilting angle of the micromirror

\subsubsection{Translation under current}

The translations of the micromirror subjected to various currents applied to electromagnets are measured and compared with the simulated results as shown in Figure 4.9a. The maximum translation is $144 \mu \mathrm{m}$ at $140 \mathrm{~mA}$ experimentally. The simulation result of the displacement at $140 \mathrm{~mA}$ is $129 \mu \mathrm{m}$. The discrepancy comes from the approximation of the moving film shape and the force distribution used in the magnetic 
force and deformation simulation. In operation, the lower corners of trapezoidal plate are further lifted from the substrate than before operation due to the repulsive magnetic force used, as shown in Figure 4.7b. The repeatability is significantly improved by separating the lower corners of trapezoidal plate from the substrate, which is $<1 \%$ while the previous design's repeatability is $14.22 \%$ due to the touching in operation. In addition, the mirror's starting position is consistent each time whereas the previous design needs to have the pre-oscillation for $20-30$ minutes before each usage to reduce the starting position variation (from $15 \mu \mathrm{m}$ to $3 \sim 4 \mu \mathrm{m}$ ) because of the touching between the moving film and substrate before operation.

The tilting of the micromirror is measured as shown in Figure $4.9 \mathrm{~b}$ and the maximum tilting is $0.3^{\circ}$ at $140 \mathrm{~mA}$. The driving circuit is shown in Figure 4.10. The micromirror requires a maximum $>100 \mathrm{~mA}$ driving current which cannot be supplied directly from a signal generator. A current amplifier is developed to drive the micromirror which can deliver up to $750 \mathrm{~mA}$ current with high input impedance. The high current amplifier circuit can work standalone with onboard potentiometer to adjust the voltage of $0-2.5 \mathrm{~V}$ or interface with computer to receive control signal.

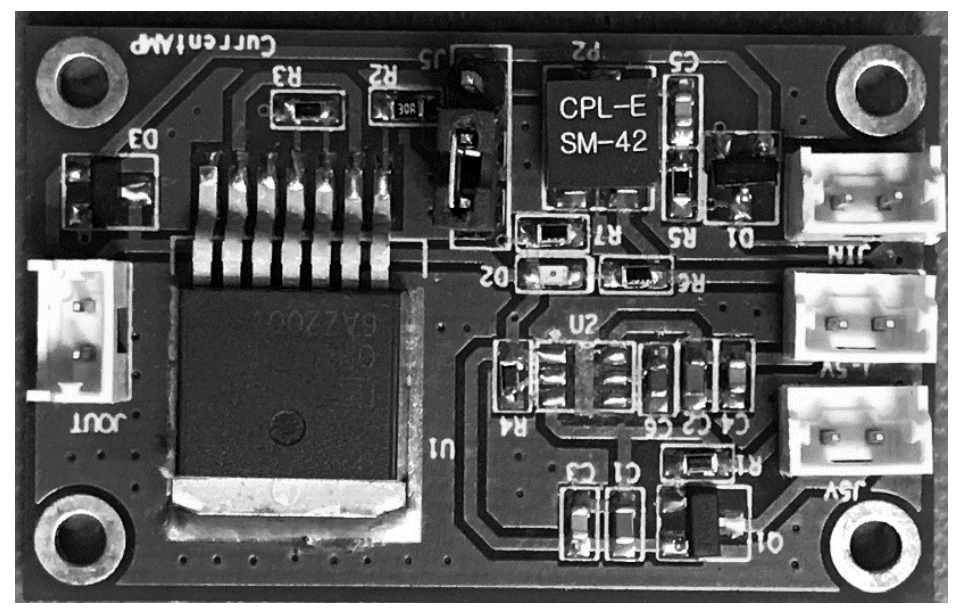

Figure 4.10 Driving circuit for micromirror [153] 


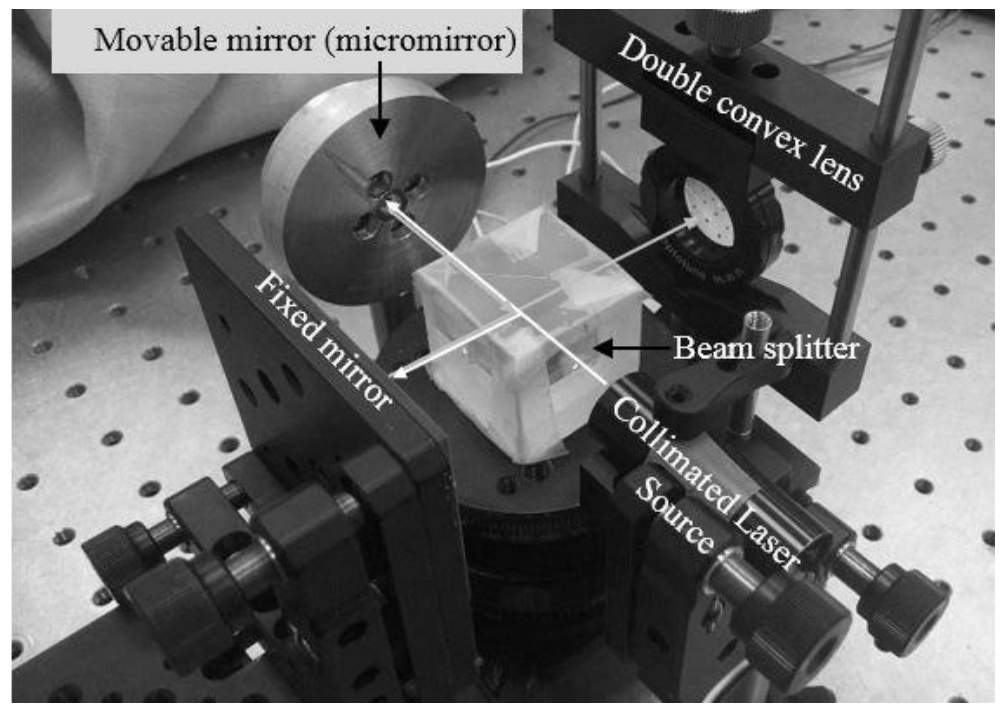

(a)
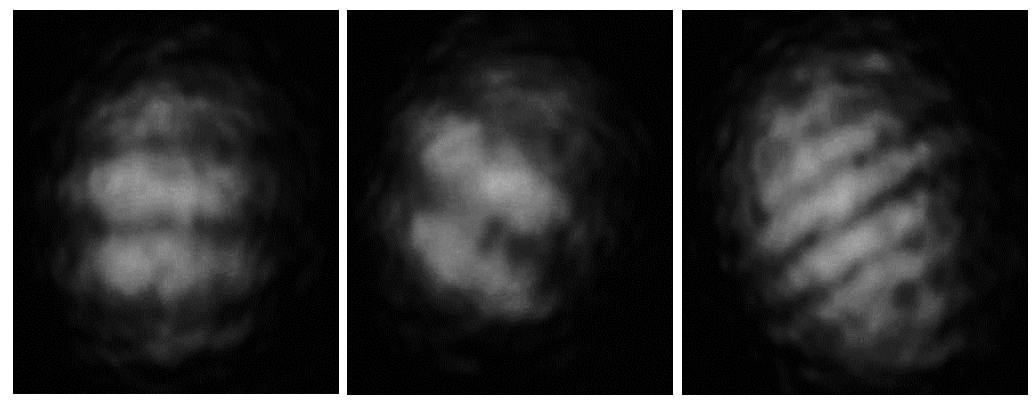

(b)

Figure 4.11 Fringes patterns appeared when the micromirror is at different displacement.

(a) Michelson interferometer with designed movable micromirror; (b) Straight fringes patterns on the screen when the moveable micromirror travels $7.2 \mu \mathrm{m}$ (the middle one) and $29.5 \mu \mathrm{m}$ (the right one) from the original position (the left one)

\subsubsection{Application: wavelength measurement}

The micromirror is used as a movable mirror to construct a Michelson interferometer to measure the wavelength of a laser beam. As shown in Figure 4.11a, the reflected and transmitted beams are redirected by the fixed and the movable mirrors to the output where they are superposed to form the interference pattern (fringes). Therefore, the following relationship holds:

or

$$
\begin{aligned}
& \delta=2 \Delta=n \cdot \lambda \\
& \delta=(n+1 / 2 \lambda)
\end{aligned}
$$


for constructive and destructive interferences [19], where $\delta$ is OPD, $\Delta$ is the displacement of the movable mirror, $\mathrm{n}$ is the number of fringes which is an integer and $\lambda$ is the wavelength of light. The wavelength can be calculated based on the displacement of the movable mirror and the number of fringes obtained. The result is affected by two factors: 1) Integerization error, only integer number of fringes are counted. Fractional number of fringes is approximated up or down to an integer number of fringes. This error can be reduced by increasing the displacement of the movable mirror; 2) The tilting associated with the translation of the micromirror limits the maximum displacement of the micromirror to be used in the measurement, beyond which the fringe pattern becomes blurred. As shown in Figure 4.11b, fringes are counted using the straight patterns which become wider when the micromirror moves with more tilting [154]. In this prototype, the translation is limited to $30 \mu \mathrm{m}$. A measurement error of $2.19 \%$ is obtained for a $532 \mathrm{~nm}$ wavelength laser beam. In addition, tilt not only changes the width of the fringes but also rotates them depending on the tilting axis of the micromirror. The tilts can be significantly reduced through a correcting micromirror, which will be presented in the next chapter. In comparison to the traditional movable mirror used in the Michelson interferometer, the micromirror presented here is simpler and more compact, and has lower cost since it does not have bearings and motors used in traditional movable mirror.

Table 4.2 Measurement result for $532 \mathrm{~nm}$ wavelength laser beam

\begin{tabular}{|c|c|c|c|}
\hline $\begin{array}{c}\text { Number of } \\
\text { fringes } \mathbf{n}\end{array}$ & $\begin{array}{c}\text { Mirror displacement } \Delta \mathbf{d} \\
(\mathrm{um})\end{array}$ & $\begin{array}{c}\text { Calculated wavelength } \boldsymbol{\lambda} \\
(\mathrm{nm})\end{array}$ & $\begin{array}{c}\text { Total error } \\
(\%)\end{array}$ \\
\hline 26 & 7.23 & 556.2 & 4.54 \\
\hline 54 & 14.68 & 543.7 & 2.19 \\
\hline 108 & 29.51 & 546.5 & 2.72 \\
\hline
\end{tabular}

\subsection{Summary}

A novel translation micromirror has been developed to eliminate the big variation in starting position of micromirror as well as to improve the low repeatability (from $14.2 \%$ to $<1 \%$ ) associated with previous translation micromirror. In the new design, the moving film is lifted up using a permanent magnet ring above the moving film to avoid touching the substrate before operation and an underneath electromagnet to generate the repulsive 
force to push the moving film up and away from the substrate in operation. In addition, the residual stress gradient is not required to curve up the structure in this design. The micromirror translates up to $144 \mu \mathrm{m}$ at $140 \mathrm{~mA}$. As an application of this design, the micromirror is used as a moveable mirror in a Michelson interferometer to measure the wavelength of a laser beam. A measurement error of $2.19 \%$ for a $532 \mathrm{~nm}$ laser beam is achieved. 


\section{CHAPTER 5 A TILT COMPENSATION SYSTEM FOR TRANSLATION MICROMIRROR}

This chapter is based on the following paper which is under review:

Y. Xue and S. He, “A tilt compensation system for translation micromirror", Advances in Mechanical Engineering.

A novel tilt compensation system is presented in this chapter to correct the tilt during the motion of the translation micromirror, so that it can work as the movable mirror in the Michelson interferometer based miniaturized FTIRs. This compensation system is not only capable to compensate for the specific micromirror tested in this chapter, but also applicable to any fixed tilting axis translation mircomirror. To realize the purpose, three requirements on the setup of the compensation system are identified, e.g. the beam incident angle and the relative locations of the translation mirror, the correcting mirror and the reflecting mirror. The compensation system including a translation micromirror is tested in a Michelson interferometer in the experiment. The result shows the tilt of the translation micromirror can be reduced to $0.026^{\circ}$ after compensation, so that it can be used as a movable mirror in the miniaturized FTIRs to measure the substances, such as SO, PO and NO molecules, with wavelength between $13.6 \mu \mathrm{m}$ and $25 \mu \mathrm{m}$, which equals to half of the mid-infrared region. The dimension of the packaged compensation system, including the translation micromirror, a correcting micromirror, a reflecting micromirror and the driven circuits, is $7 \mathrm{~cm} \times 4 \mathrm{~cm} \times 3.7 \mathrm{~cm}$, which is acceptable for a miniaturized FTIRs.

\subsection{Tilt compensation system design}

\subsubsection{Tilt compensation conditions}

The developed compensation system includes the translation mirror M1, a correcting micromirror M2 and a reflecting mirror M3. When a beam split by the beam splitter reaches $\mathrm{M} 1$, it is reflected to $\mathrm{M} 2$ and then $\mathrm{M} 3$. If M1 tilts during the motion, the correcting mirror $\mathrm{M} 2$ rotates a correspondent angle to compensate the tilt. With the compensation, the beam is reflected by M3 to the beam splitter through M2 and M1 to 
combine with the beam reflected from the fixed mirror to form the interference pattern. In order to achieve this objective, the system must meet the following conditions: 1) The reflected beam from M2 needs to be injected vertically to M3. Otherwise, the beam reflected from M3 cannot return to M2 along the incoming path. 2) The rotating axis of M2 must be parallel to the tilting axis of M1.3) M2 needs to rotate the same angle with the tilt of M1 to keep the angle between them consistent, as shown in Figure 5.1a. Only when all three conditions are met, the beam can fully return to the beam splitter along the incident path, so that the impact on the interference pattern from the tilt of M1 can be eliminated. Figure 5.1b shows a setup in which M3 is perpendicular to the fixed mirror and M2 is parallel to M1. Under this condition that the fixed mirror is parallel to the image of the movable mirror, the circular fringes are generated which is similar in the traditional Michelson interferometer, as shown in Figure 5.2a [155]. Otherwise, straight fringes are generated when the movable mirror is at an angle with the fixed mirror, as shown in Figure 5.2b.

\subsubsection{System setup}

The components of the compenstaion system need to be set up accoridng to the three conditions to achieve the compensation purpose. At the first step, M3 needs to be perpendicular to the reflected beam from M2 before the current is applied, which can be easily achieved by adjusting M3 until the beam spots reflected from M1 and M3 completely overlap on M2.

The second step is to parallelize the axes of M1 and M2, before which the tilting axis of M1 needs to be found out. A method is developed to find out this axis by observing the trajectory on a PSD which is set up vertically on the table, as shown in Figure 5.3. If the tilting axis is not parallel to the Z-axis, the trajectory on the PSD is not horizontal to the $\mathrm{X}$-axis, as shown in Figure 5.3a. Rotating M1 along Y-axis until its trajectory is horizontal, as shown in Figure 5.3b, then the trajectory on PSD is caused by the constructive superpose of the tilt and the translation of M1 and the tilting axis is parallel to the Z-axis which is perpendicular to the table. 


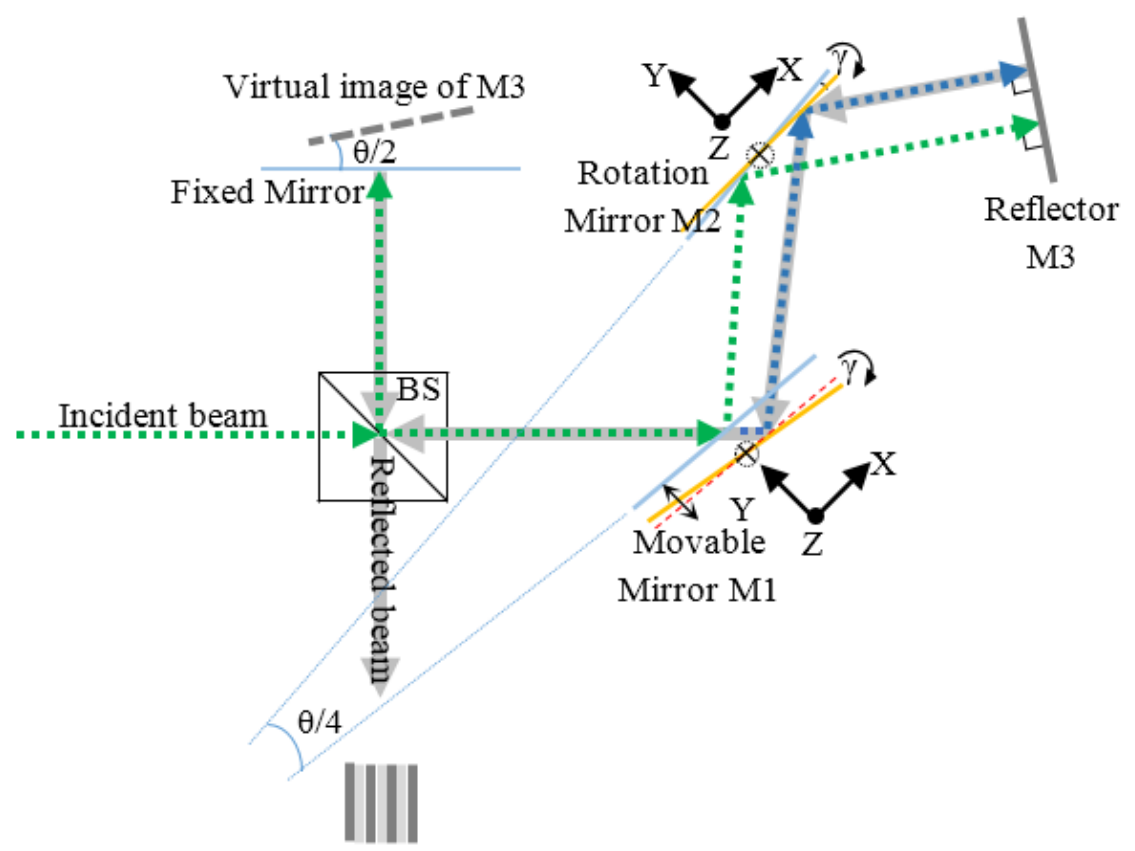

(a)

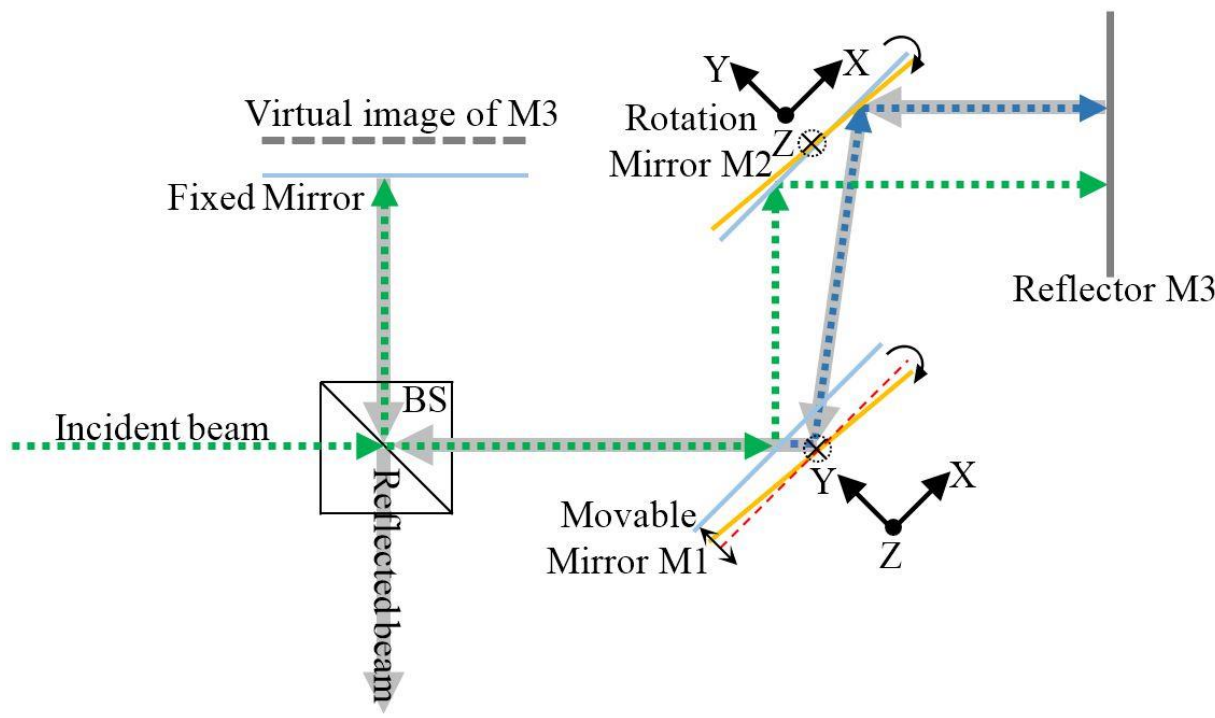

(2)

(b)

Figure 5.1 The tilt compensation system. (a) When M3 angled with the fixed mirror; (b) when M3 is perpendicular to the fixed mirror. 


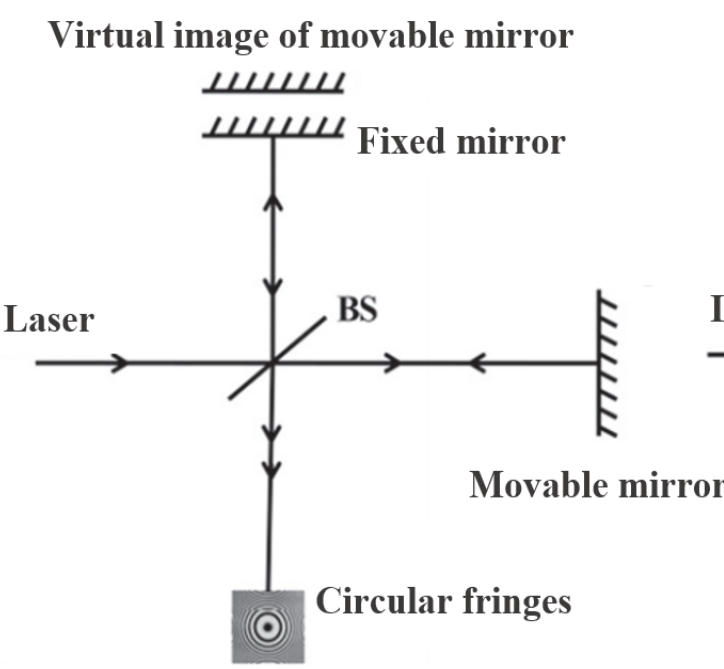

(a)
Virtual image of movable mirror

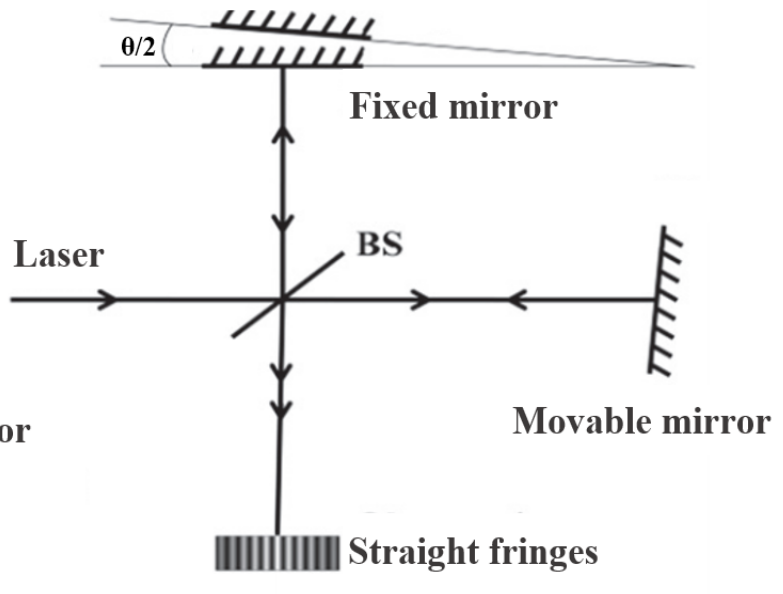

(b)

Figure 5.2 Different shapes of interference fringes generated by a Michelson interferometer. a) Circular fringes; b) Straight fringes [155]

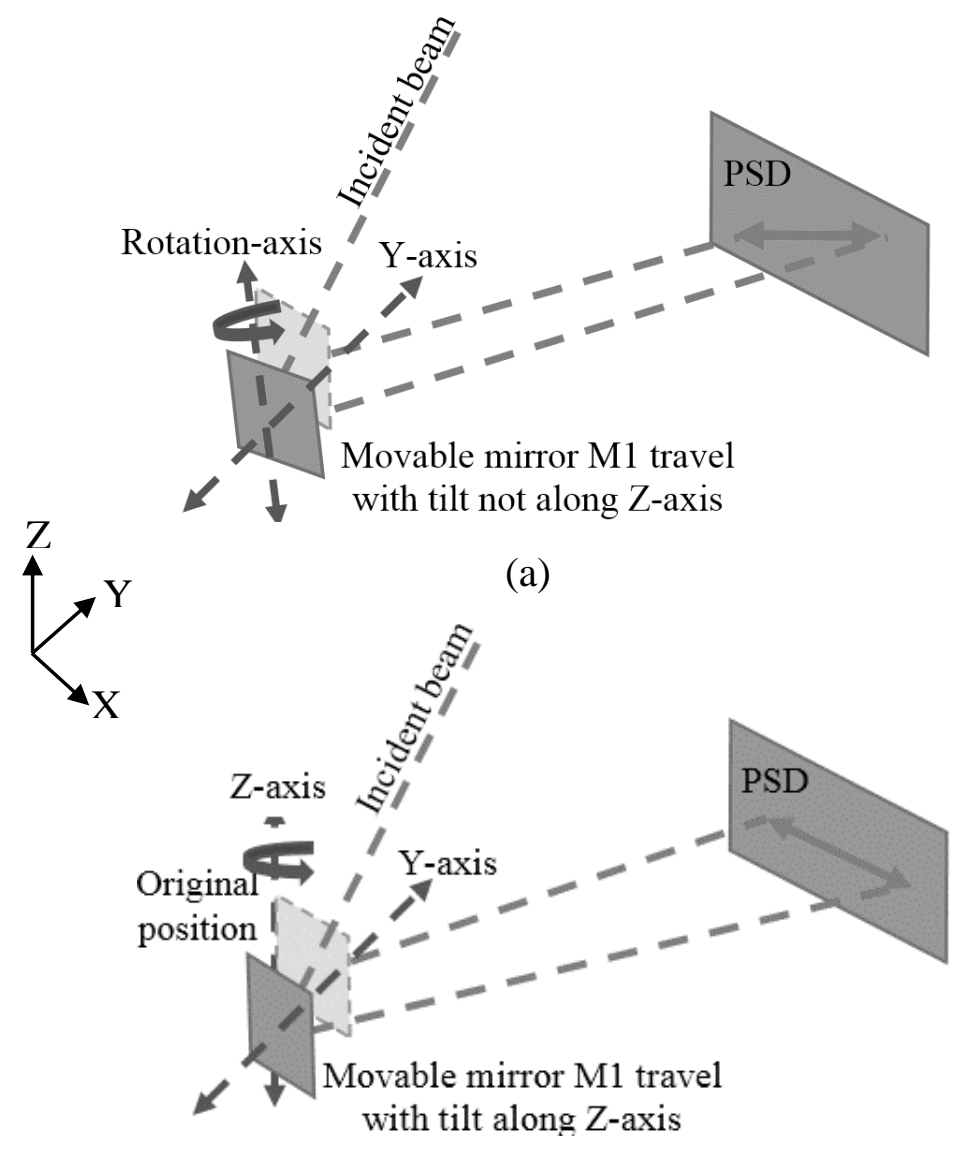

(b)

Figure 5.3 Determination of tilting axis. a) Micromirror tavels on the Y-axis with tilt along a rotation axis other than the $\mathrm{Z}$-axis; b) with tilting along the $\mathrm{Z}$-axis 
And the third step is to keep the angle between M1 and M2 consistent when M1 tilts during the motion. Before the current is applied, an initial angle $\theta$ between the reflecting beams from the fixed mirror and M3 can be obtained basing on the interference fringe width using the equation below [156]:

$$
\sin \theta=\frac{2 \lambda}{\Delta \mathrm{X}}
$$

where $\lambda$ is the wavelength of the beam and $\Delta \mathrm{X}$ is the width of the interference fringe. When the current is applied on M1, the tilt of M1 leads to the misalignment of two beams, then the width of the interference fringes changes. Therefore, a current needs to be applied on M2 to correct the tilt and a new angle $\theta^{\prime}$ is obtained. Ideally, the fringes width after compensation should be infinitely close to the initial fringe width and, correspondingly, the compensated angle $\theta^{\prime}$ is close to the initial angle $\theta$. Then the compensation capacity of the system can be determined by the difference $\Delta \theta$ between $\theta$ and $\theta^{\prime}$. In order to find $\theta^{\prime}$, a trial-and-error method is used to find the current applied on M2 to compensate the tilt of M1. The detailed steps are as following: 1) An interference pattern is obtained as a reference after setting up all the mirrors before the current is applied; 2) M1 displaces with the tilt after a static current is applied, which leads to the interference pattern change; 3) Multiple interference patterns are generated by applying a series of currents on M2, then one pattern similar to the reference is chosen and the corresponded current is recorded. This process is repeated until all the compensation currents on M2 are found for each critical displacement of M1 to complete the calibration. A linear interpolation method can be used to estimate the current on M2 to compensate the tilt of M1 at other displacements. A table is generated to record each displacement of M1 and the correspondent current pairs on M1 and M2. Then the calibration process and the equipment, such as the PSD and the stages, are no longer required. The data in the table can be input to the open-loop control directly in the real operation. 


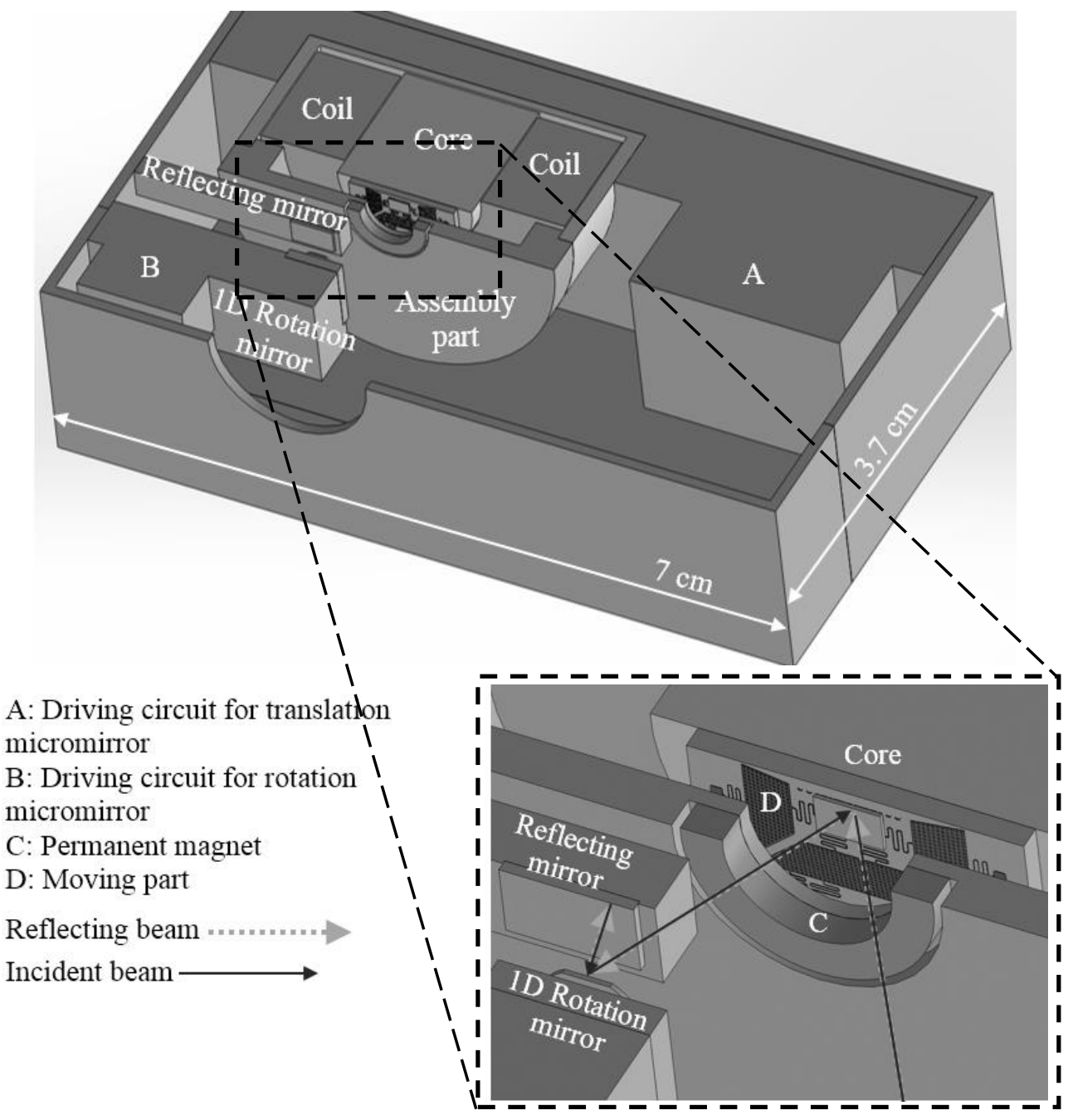

Figure 5.4 Packaged tilt compensation system with a re-designed assembly part for repulsive magnetic force translation micromirror

\subsubsection{Size consideration}

The size of the compensation system is important for the miniaturized interferometer. As shown in Figure 5.4, the packaged compensation system, which includes the assembled repulsive force translation micromirror [153], a 1D rotation micromirror, a reflecting mirror, and the driven circuits, has a size of $7 \mathrm{~cm}$ (length) $\mathrm{x} 3.7 \mathrm{~cm}$ (width) $\mathrm{x} 4$ $\mathrm{cm}$ (height), in which the rotation and the reflecting mirrors are both $5 \mathrm{~mm} \times 5 \mathrm{~mm}$. Since the tilt angle of most translation micromirrors is small, e.g. $< \pm 2^{\circ}$, a micromirror with a single axis and small rotation angle can satisfy. This type of rotation micromirror is commercially available, such as [157]-[159], which can provide the rotation angle $\pm 6^{\circ}$ $\pm 30^{\circ}$ in static and dynamic mode and the high surface quality $(<10 \mathrm{~nm}$ surface roughness 
and $>5 \mathrm{~m}$ curvature). Though using the compensation system increases the size, the overall size is still more advantageous over the conventional interferometer. In addition, the miniaturized interferometer has relatively simple structure and low cost due to batch production.

\subsection{Experiments}

\subsubsection{Platform setup}

Michelson interferometer with the tilt compensation system is setup as shown in Figure 5.5a, which includes a laser $(5 \mathrm{~mW}, 532 \mathrm{~nm})$, a beam splitter, a fixed mirror, and the tilt compensation system. The repulsive force micromirror does not have the touching issue, so the problem existing with the attractive force translation micromirror is avoided [90], such as the variation of the starting position and low repeatability. However, the prototype of the repulsive force micromirror in [153] is not suitable for the tilt compensation system, e.g., the ring shape permanent magnet is far from the mirror plate, so the angle between the incident and reflecting beam is too small and the optical path is affected. Therefore the attractive force translation micromirror is used as M1 in the experiment. Since the purpose of this chapter is to develop a method of tilt compensation for the translation micromirror, the attractive force micromirror can be used in the experiment for the purpose. Figure 5.5b shows the components and their setup in the compensation system. M1 travels under the magnetic field generated by the electromagnet underneath it, as shown in Figure 5.5c. M2 is driven by the Lorentz force applied on the FPCB [160] [161] underneath the mirror plate. The Lorentz force is generated by two permanent magnets on the side when the current passes through the magnetic field, as shown in Figure 5.5d. M1 is placed on a rotation stage, as shown in Figure 5.5, both of which are then mounted on a 2D linear stage, so M1 can move in the $\mathrm{XY}$ plane. M2 is mounted with its rotating axis perpendicular to the table on a 1D linear stage [162] to move in the vertical direction. Therefore, the relative position between M1 and M2 can be adjusted to make the tilting axis of M1 parallel with the rotation axis of M2 and the beam can be reflected by M1 to reach M2. The laser, the fixed mirror and M3 are mounted on the tip and tilt adjustment stages to ensure the reflected beams can combine at the beam splitter. All the parts including the beam splitter and the concave lens are assembled on a steel breadboard to ensure the stability of the system. In order to 
ensure the reflected beam from M2 injects vertically to M3, M3 is adjusted so that the reflected beam from it overlaps with the incident beam on M2. Since multiple stages are used to adjust the positions of the mirrors in the calibration stage, the size of the experiment setup is large, but the real package will be much smaller after the calibration is completed and the stage are removed.

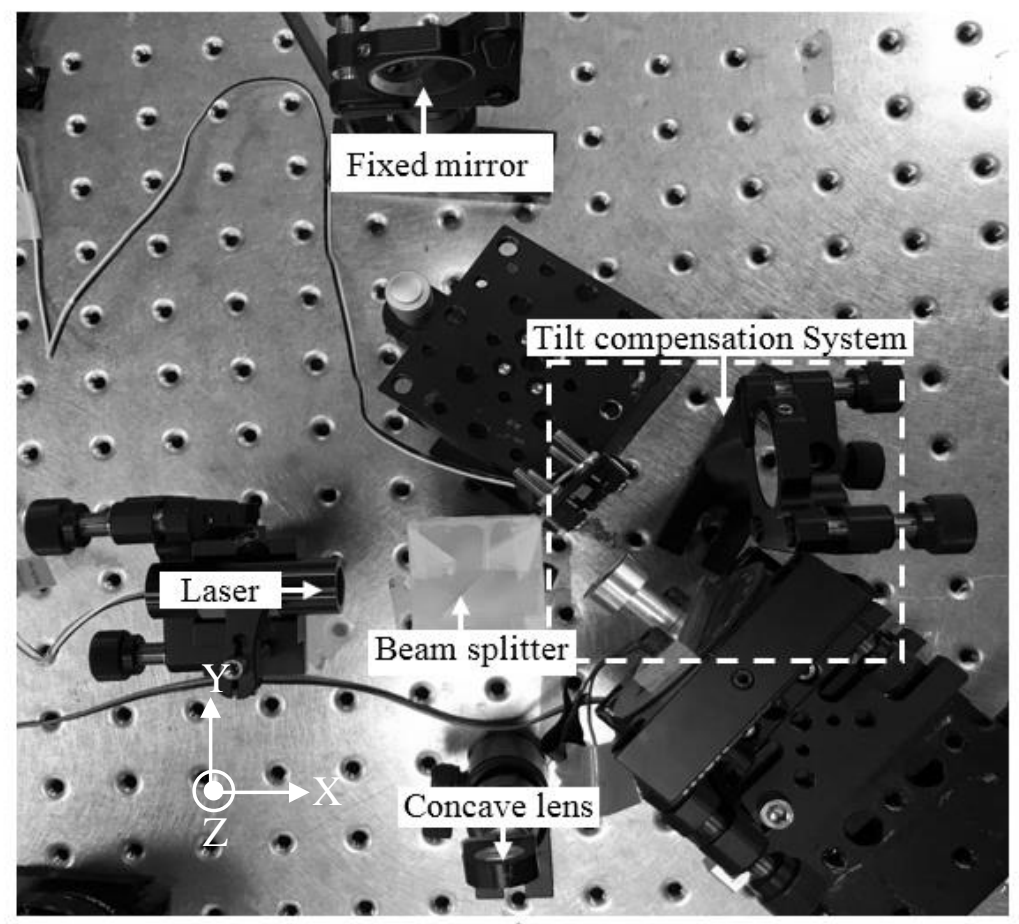

a)

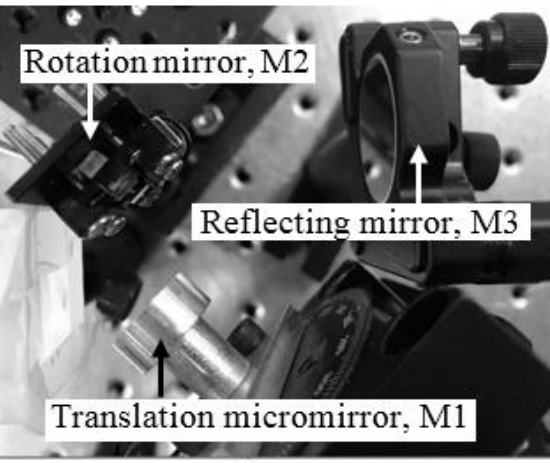

b)

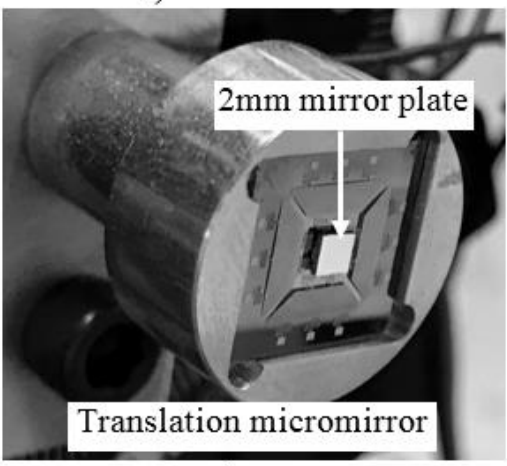

c)

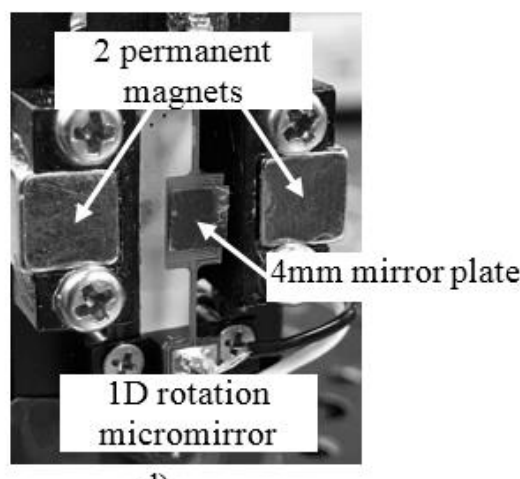

d)

Figure 5.5 Michelson interferometer with a tilt compensation system; (a) Top view of the experimental platform; (b) Detailed view of tilt compensiton system; (c) Detailed view of the movable mirror M1; (d) Detailed view of the 1D rotation mirror M2. 


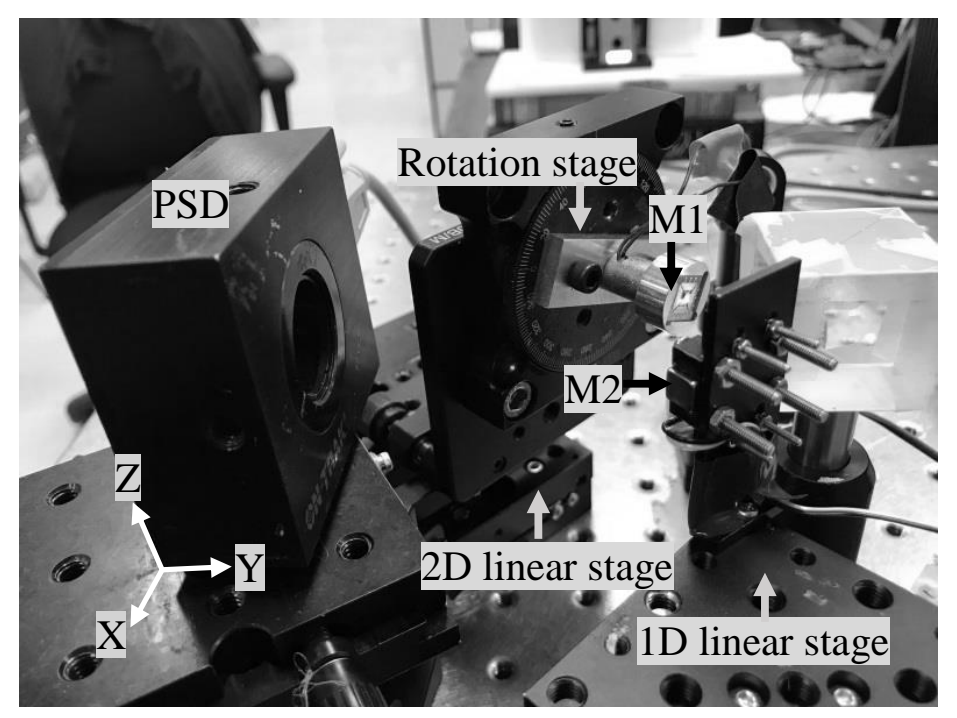

(a)

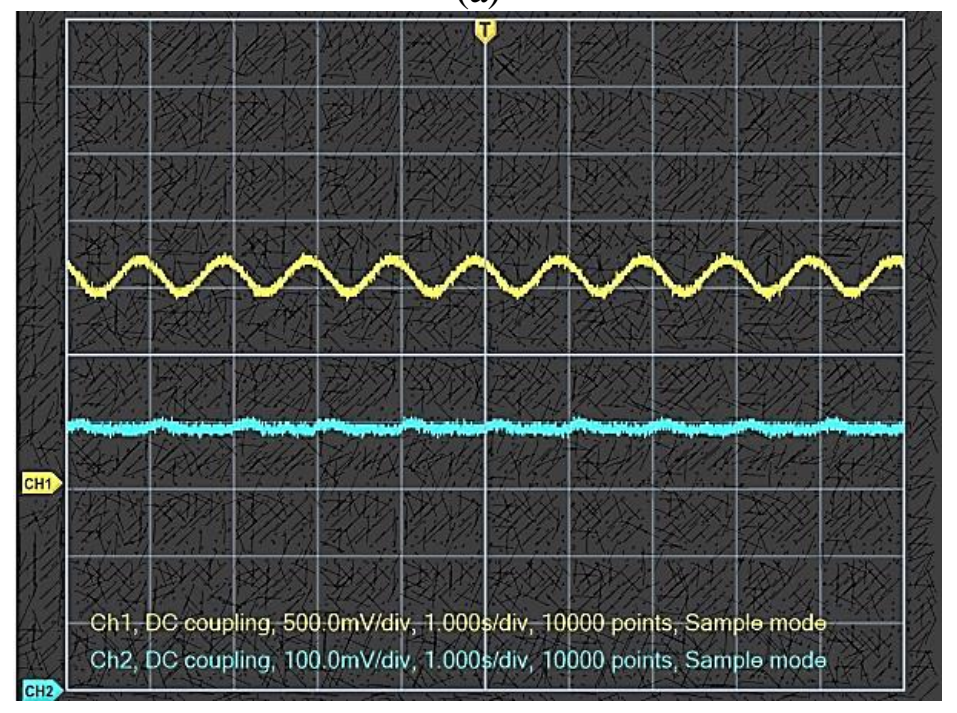

(b)

Figure 5.6 Determine the tilting axis using the PSD and the oscilloscope. (a) Experiment setup; (b) The minimum voltage $(<10 \mathrm{mV})$ is observed when $\mathrm{M} 1$ rotates $72^{\circ}$ and the displacement is around $100 \mu \mathrm{m}$

\subsubsection{Determine the tilting axis}

As introduced in Section 5.1.2, the tilting axis of M1 can be found using an oscilloscope to monitor the change of the trajectory on the PSD, as shown in Figure 5.6. Figure 5.6a shows M1 is on a rotation stage [163] which rotates along Y-axis and M2 and a PSD are placed vertically on the table. When a laser is injected to the PSD after reflected by the mirrors, M1 is rotated until the minimum voltage is found in the 
oscilloscope channel in the vertical direction of the PSD. For example, $10 \mathrm{mV}$ voltages is observed on the oscilloscope (the blue line in Figure 5.6b) when M1 travels about 100 $\mu \mathrm{m}$. This is the minimum voltage found on the oscilloscope after M1 rotates $360^{\circ}$ along the $\mathrm{Y}$-axis, which means the tilting axis of $\mathrm{M} 1$ is approximately perpendicular to the table at this position.

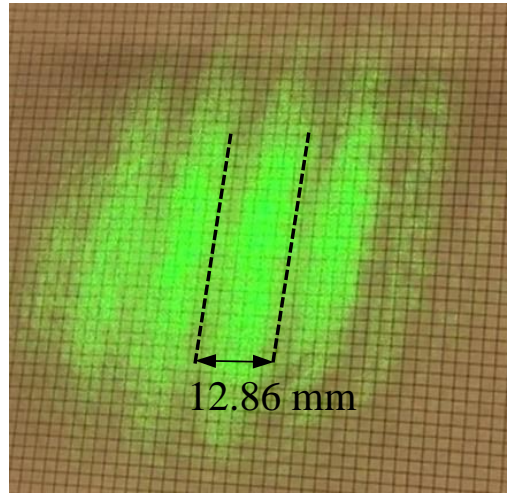

(a)

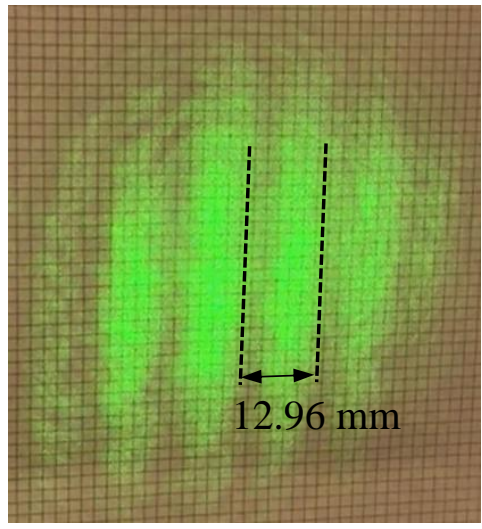

(d)

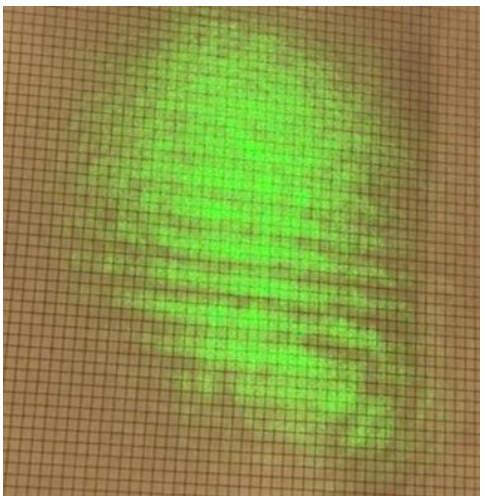

(b)

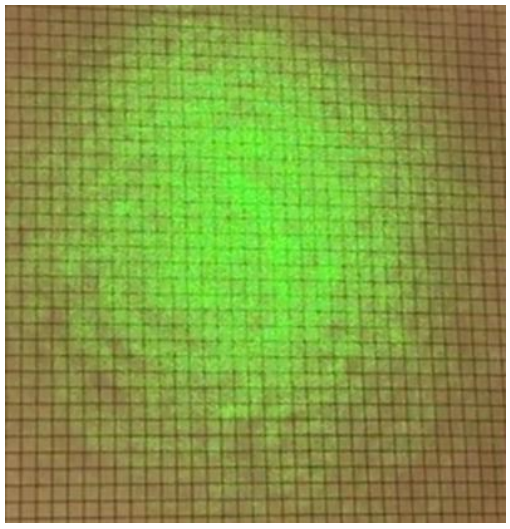

(e)

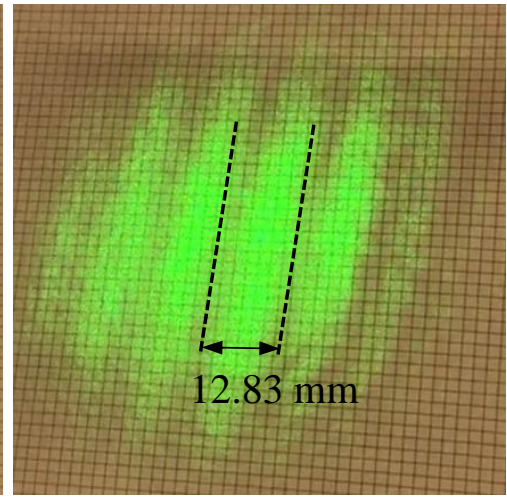

(c)

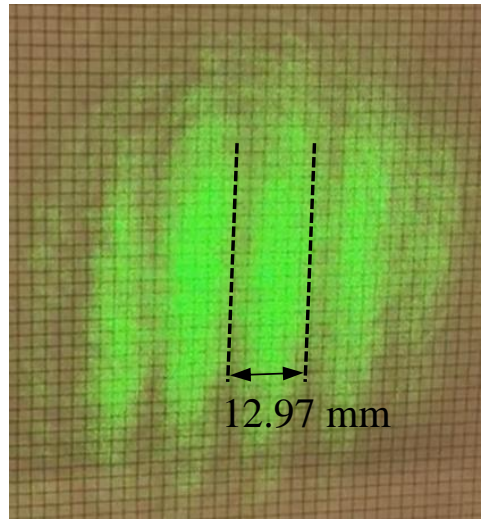

(f)

Figure 5.7 Interference pattern on the screen. a) $1^{\text {st }}$ reference fringes; b) Spots misaligned under $30 \mu \mathrm{m}$ displacement without compensation; c) Interference pattern when M1 travels $30 \mu \mathrm{m}$ with compensation; d) $2^{\text {nd }}$ reference fringes; e) Only the spot from the fixed mirror is shown on the screen due to no compensation; f) Interference pattern when M1 travels over $100 \mu \mathrm{m}$ with compensation

\subsubsection{Tilt compensation by M2}

After all components are assembled according to the conditions, the interference pattern generated by the reflected beams from the fixed mirror and M3 is shown on the 
screen, where a $2 \mathrm{~mm}$ grid paper is pasted for measurement purpose, as shown in Figure 5.7. A reference pattern is measured, as an example shown in Figure 5.7a, in which 4 fringes are on the screen at the starting position when no current is applied on M1, and then the pattern is imported to the computer. Two lines are drawn manually along the edges between two adjacent dark or bright stripes and the distance between them is measured. The edges are currently identified by eyes but the distance can be measured more accurately by using the computer aided grayscale mapping solution. After the distance between the lines is measured, the fringe width can be calculated by scaling with the grids.

A current is applied on M1 after the reference pattern is generated. For example, after M1 displaces $30 \mu \mathrm{m}$, the pattern on the screen is misaligned due to tilt, as shown in Figure 5.7b. To compensate it, a trial-and-error method is used to find the required current on M2. A series of currents with $1 \mathrm{~mA}$ incremental every 10 milliseconds are applied. Among the generated interference patterns under different currents, the pattern most similar to the reference is identified and the correspondent current is recorded. For example, when M1 travels $30 \mu \mathrm{m}$, the most similar pattern is found when $15 \mathrm{~mA}$ current is applied on M2, as shown in Figure 5.7c.

Because the limitation of the attractive force micromirror leads to $3 \sim 4 \mu \mathrm{m}$ starting position variation on M1 even after 30 minutes pre-oscillation, the pattern cannot return to the starting position completely. Therefore, the initial interference pattern needs to be measured again as the new reference for each independent experiment. For example, as shown in Figure 5.7d-f, when M1 travels $100 \mu \mathrm{m}$, the new reference is in Figure 5.7d and $45 \mathrm{~mA}$ current is found on M2 as shown in Figure 5.7f. By comparing with the previous reference in Figure 5.7a, the fringe width changes $0.1 \mathrm{~mm} .12$ equal incremented currents are applied on M1 until the rated current is reached, then the correspondent currents on M2 are obtained following the process and recorded. 

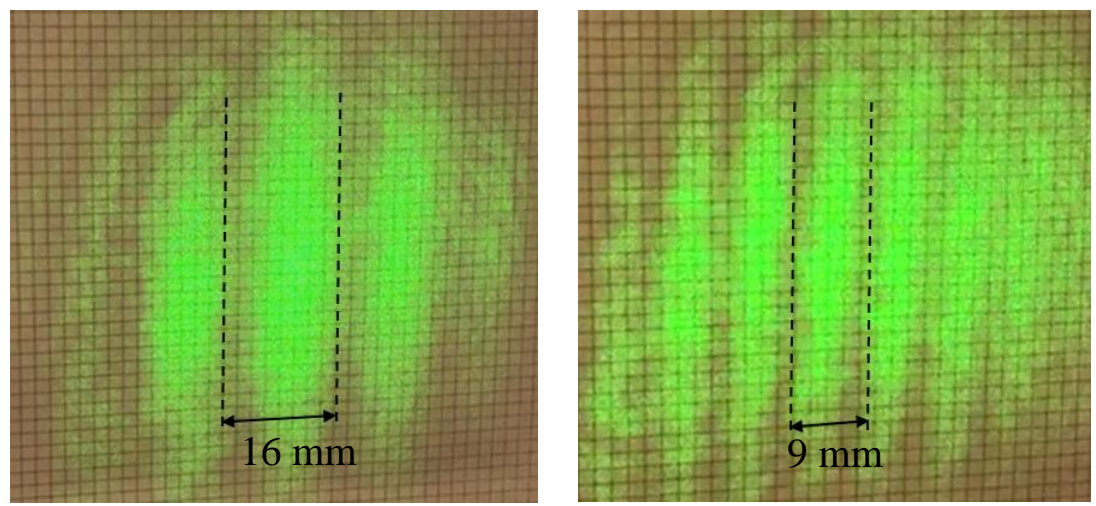

Figure 5.8 Interference firinge change due to the tilt of M1

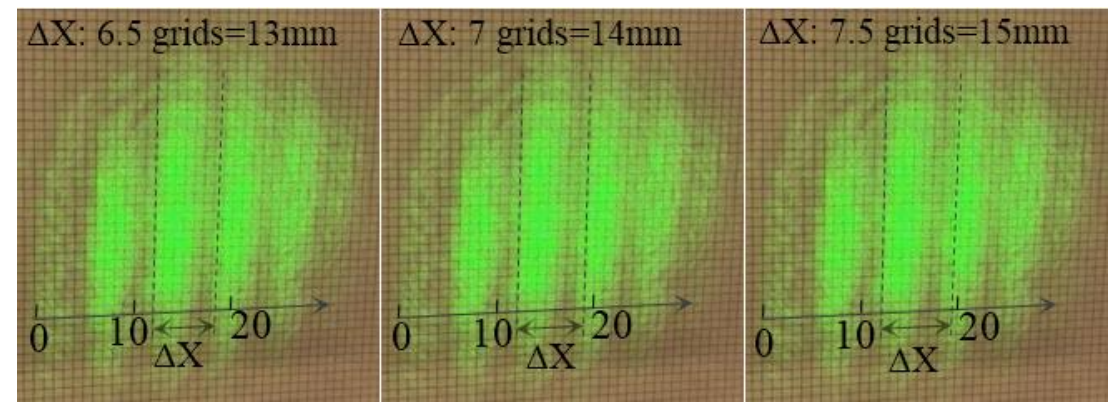

(a)

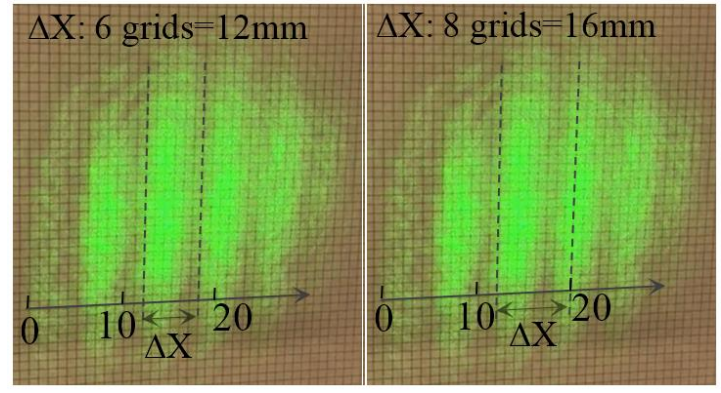

(b)

Figure 5.9 Fringe width on the same interference pattern. (a) Tolerable error range (14 $\mathrm{mm} \pm 1 \mathrm{~mm}$ ); (b) Out of the tolerable range because the line is in the mid of the dark (left) or bright (right) stripe

\subsection{Data analyses}

\subsubsection{Error of the fringe width}

To verify equation 5.1 can be used to obtain the tilt angle and find the error between the caculation and measuramnet reuslt, an example is used as shown in Figure 5.8. The initial fringe width is $16 \mathrm{~mm}$ and the angle $\theta$ is $0.13^{\circ}$. When $67 \mathrm{~mA}$ current is applied on 
the translation mirormirror, the fringe width changes to $9 \mathrm{~mm}$ and the corresponding angle $\theta^{\prime}$ is $0.235^{\circ}$. So the difference between these two angles, which is the tilt, is $0.105^{\circ}$ that is very close to the $0.1^{\circ}$ measurment result using Zygo optical profile machine under the same current. However, the error in current compensation system is mainly caused by the inaccurate fringe width measurement on the screen. Because the edges are identified by eyes and the margins between fringes are not clear on the interference pattern, the fringe width varies at each measurement. As shown in Figure 5.9, five different fringe widths are measured on the same interference pattern. Only $12 \mathrm{~mm}$ and $16 \mathrm{~mm}$, as shown in Figure 5.9b, can be easily excluded. The range from $13 \mathrm{~mm}$ to $15 \mathrm{~mm}$, as shown in Figure 5.9a, could be identified as the fringe width which results a measurement error of $\pm 1 \mathrm{~mm}$, so the calculated angle $\theta$ is in a range, e.g., $0.08^{\circ} \sim 0.13^{\circ}$ instead of $0.105^{\circ}$. This result is not accurate, but veirifies that the angle before and after compensation can be obtained using equation 5.1. Due to the measurement error, the maximal difference between the range of $\theta$ and $\theta^{\prime}$ is considered as $\Delta \theta$. For example, $\theta_{\max }$ is calculated using equation 5.1 when the fringe width in the reference is $13.86 \mathrm{~mm}(12.86+1)$ and $\theta^{\prime}{ }^{\prime}$ in is $11.83 \mathrm{~mm}(12.83-1)$ after compensation, $\Delta \theta$ is $0.026^{\circ}$. As shown in Figure 5.10, $\Delta \theta$ is calculated at 12 different points, among which $0.026^{\circ}$ is the maximum value and considered as the capability of the compensation system.

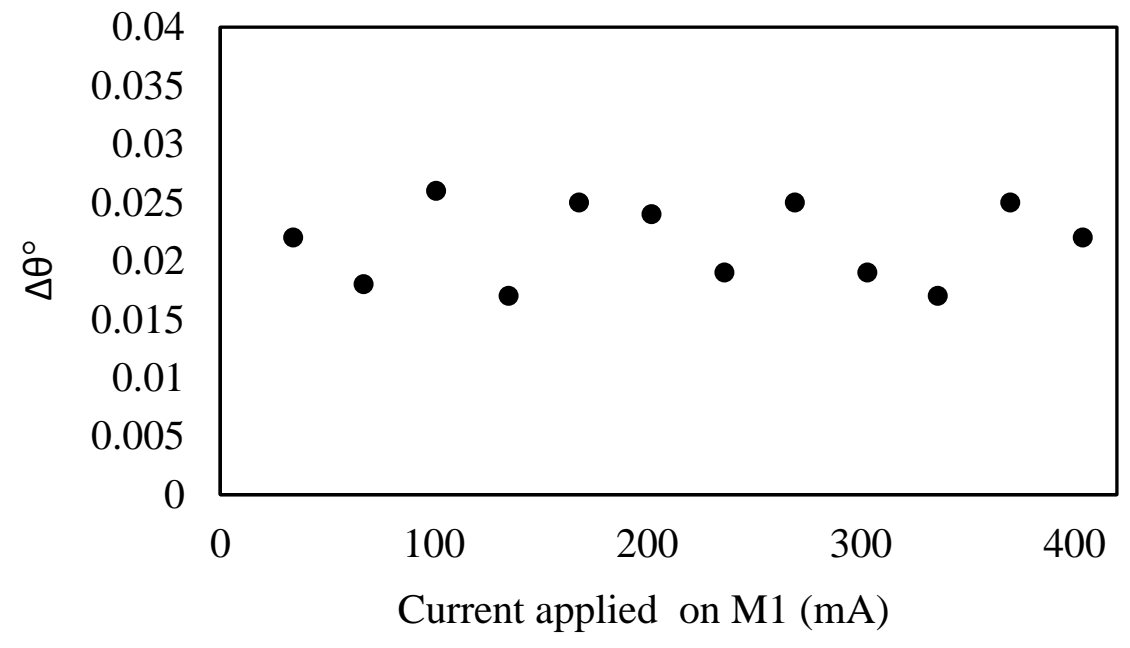

Figure 5.10 Angle difference $\Delta \theta$ 


\subsubsection{Interference pattern shifts caused by the variation of the titling axis}

The tilting axis of the attractive translation microminor actually has a variation around $3^{\circ}$ basing on previous measurement result, which indicates that the tilting axis can incline up to $\pm 1.5^{\circ}$. The impact of the tilting axis variation on the compensation result needs to be analyzed, as shown in Figure 5.11. For a beam reflected from M1, it is injected on the beam splitter at $A$ if no tilt exists so that it can fully combine with the reflected beam from the fixed mirror on the beam splitter. When M1 tilts angle $\varphi$ along the Z-axis, the spot on the beam splitter is moved to $A^{\prime}$. And the spot moves to $B^{\prime}$ 'if the M1 tips an angle $\omega$ out of the X-Y plane. Under this situation, the spot moves to $B$ instead of $A$ using the compensation system. The location variation of the beam spots on the beam splitter has the relationship below:

$$
\begin{aligned}
x & =\tan \varphi \cdot l \\
V_{S} & =\tan \omega \cdot x \\
H_{S} & =x \cdot(1-\cos \omega)
\end{aligned}
$$

where $l$ is the distance between M1 and $A, x$ is the distance between $A$ and $A^{\prime}, V_{s}$ and $H_{s}$ are the shear of the interference pattern on the vertical and horizontal direction of the screen, respectively. For the attractive force actuator [6], $\omega$ is $3^{\circ}, \varphi$ is $0.24^{\circ}$ at its maximum displacement. The distance $l$ between $\mathrm{M} 1$ and the beam splitter is $25 \mathrm{~mm}$ in this setup. Basing on equation 5.2 - 5.4, $x$ can be calculated to be $130.9 \mu \mathrm{m}, V_{\mathrm{s}}$ is 6.86 $\mu \mathrm{m}$ and $H_{s}$ is only $0.1794 \mu \mathrm{m}$ which is much smaller than $V_{\mathrm{s}}$. In addition, the vertical shear will only impact the interference pattern when it is close to the diameter of the light source because one beam becomes above the other and the interference cannot happen [164]. Therefore, the change of the tilting axis can be ignored. 


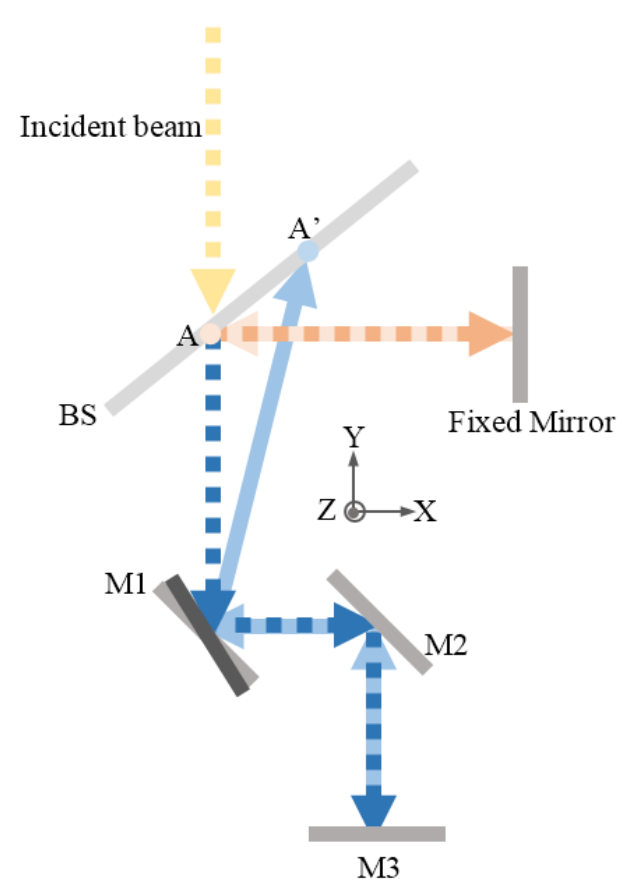

(a)

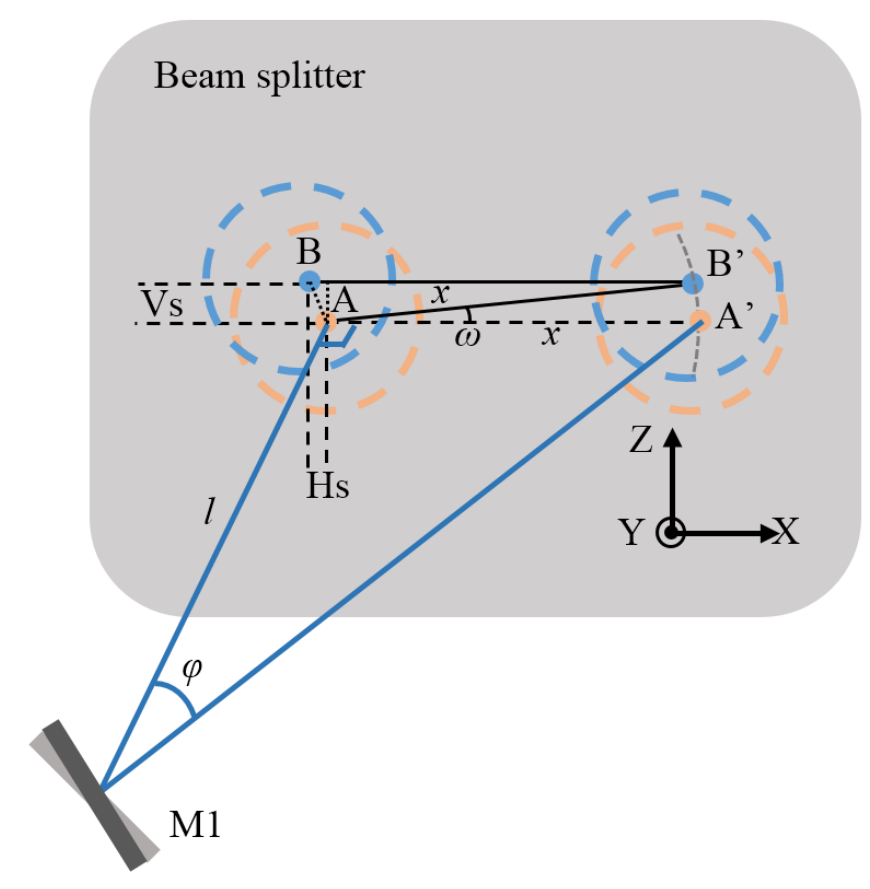

(b)

Figure 5.11 Tilt analysis. (a) Top view of the compensation system; (b) Side view of the interference pattern on the screen

\subsubsection{Application}

In a Michelson interferometer based FTIRs, the maximum tilt $\beta$ of the movable mirror without degrading the resolution of the interferometer needs to satisfy the following relationship [165]:

$$
\beta<\frac{\lambda}{20 D}
$$

where $\mathrm{D}$ is the diameter of the beam and $\lambda$ is the wavelength, as shown in Figure 5.12. Since $\mathrm{D}$ is a constant once the light source is chosen, $\beta$ is proportional to the wavelength. For example, to measure a sample in the mid-infrared region $(\lambda: 2.5 \mu \mathrm{m}-25 \mu \mathrm{m})$, the tilt angle $\beta$ must be less than $0.005^{\circ}-0.05^{\circ}$ when $\mathrm{D}$ is $1.5 \mathrm{~mm}$. Therefore, the attractive translation micromirror with the developed compensation system can be used in a miniaturized FTIRs to detect substances in the infrared region above $13.6 \mu \mathrm{m}$ (wavefront $735 \mathrm{~cm}^{-1}$ ), such as SO, PO and NO [166] as per Figure 5.13. 


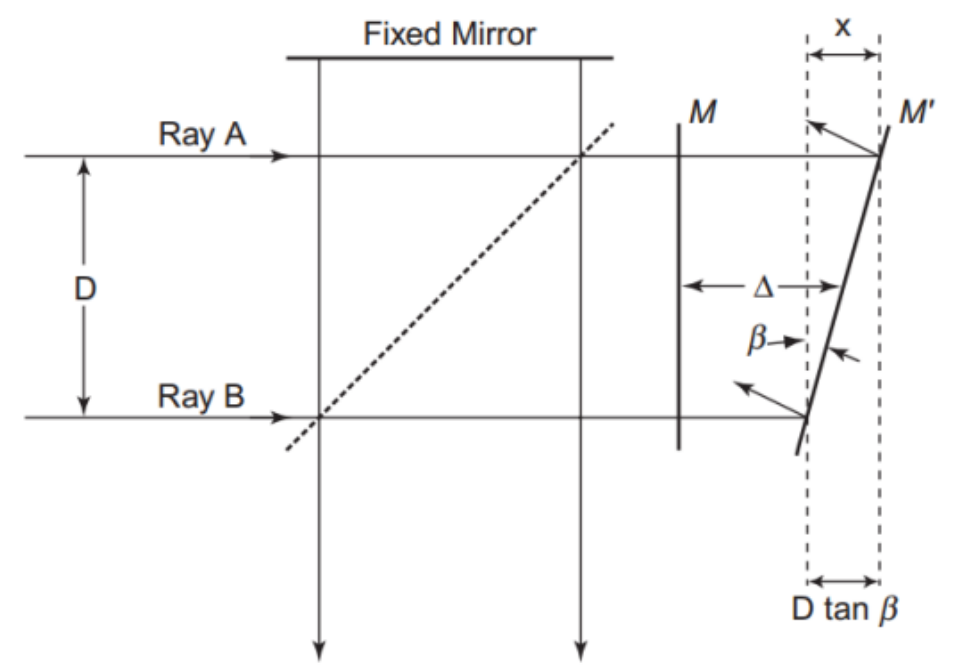

Figure 5.12 The movable mirror tilts $\beta$ with displacement

\begin{tabular}{|c|c|c|c|}
\hline Species & Source & Wavenumber $\left(\mathrm{cm}^{-1}\right) *$ & Wavelength $(\mu \mathrm{m})$ \\
\hline \multirow[t]{4}{*}{$\mathrm{CO}_{2}(\mathrm{~g})$} & Atmospheric & $\sim 4870-5000(\mathrm{~s})$ & $2.01,2.06$ \\
\hline & & $\sim 6250$ (w doublet) & $\sim 1.6$ \\
\hline & & 2350 (vs- doublet) & $\sim 4.25$ \\
\hline & & $667(\mathrm{~m})$ & 15.0 \\
\hline \multirow{2}{*}{$\mathrm{H}_{2} \mathrm{O}(\mathrm{g})$} & Atmospheric & $3900-3400$ (vs) & $2.6-2.9$ \\
\hline & & $1850-1350(\mathrm{~s})$ & $5.4-7.4$ \\
\hline $\mathrm{SO}_{2}(\mathrm{~g})$ & Atmospheric, anthropogenic & $1140-1080(\mathrm{~s})$ & $8.8-9.3$ \\
\hline \multirow[t]{2}{*}{$\mathrm{O}_{3}(\mathrm{~g})$} & Atmospheric troposphere, & $\sim 1042(\mathrm{~s})$ & $\sim 9.6$ \\
\hline & anthropogenic & $\sim 28500(\mathrm{vs})$ & $\sim 0.35$ \\
\hline $\mathrm{O}_{2}(\mathrm{~g})$ & Atmospheric & $\sim 13100(\mathrm{~s})$ & 0.76 \\
\hline \multirow[t]{3}{*}{$\mathrm{Si}-\mathrm{O}$} & Aerosol- soil & $1110-1000(\mathrm{~s})$ & $9.0-10.0$ \\
\hline & & $805(\mathrm{~m})$ & 12.4 \\
\hline & & $450(\mathrm{~m})$ & 22.2 \\
\hline \multirow[t]{2}{*}{$\mathrm{S}-\mathrm{O}$ in $\mathrm{SO}_{4}{ }^{2-}$} & Aerosol- salt & $1140-1080(\mathrm{vs})$ & $8.8-9.3$ \\
\hline & & $680-610(\mathrm{~m})$ & $14.7-16.4$ \\
\hline \multirow[t]{3}{*}{$\mathrm{C}-\mathrm{O}$ in $\mathrm{CO}_{3}{ }^{2-}$} & Aerosol- salt & $1510-1410$ (vs) & $6.6-7.10$ \\
\hline & & $880-860(\mathrm{~m})$ & $11.4-11.6$ \\
\hline & & 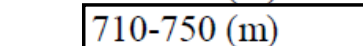 & $14.1-13.3$ \\
\hline \multirow{2}{*}{$\mathrm{P}-\mathrm{O}$ in $\mathrm{PO}_{4}^{3-}$} & Aerosol- salt, anthropogenic & $1100-1000(\mathrm{~s})$ & $9.1-10.0$ \\
\hline & & $600-500$ & $16.7-20.0$ \\
\hline $\mathrm{N}-\mathrm{H}$ & Biologic and anthropogenic & $3500-3200$ & $2.9-3.1$ \\
\hline $\mathrm{N}-\mathrm{O}$ in $\mathrm{NO}_{3}{ }^{-}$\& & Biologic and anthropogenic & $1400-1340$ (vs) & $7.1-7.5$ \\
\hline \multirow[t]{2}{*}{$\mathrm{N}_{2} \mathrm{O}$} & & $840-810(\mathrm{~m})$ & $11.9-12.4$ \\
\hline & & $720(\mathrm{~m})$ & 13.9 \\
\hline $\mathrm{C}-\mathrm{H}$ & $\mathrm{CH}_{4}$, biologic and fingerprints & $3200-2800(\mathrm{~s})$ & $3.1-3.6$ \\
\hline $\mathrm{Si}-\mathrm{CH}_{3}$ & Si-based grease and oils & $1270(\mathrm{~s})$ & 7.9 \\
\hline
\end{tabular}

Aerosol data from Berner \& Berner (1996) and wavenumbers for compounds from Smith (1999) and Clark

Figure 5.13 Spectral Range [166] 


\subsection{Summary}

A novel tilt compensation method is introduced in this chapter, which is designed to reduce the tilt of the translation micromirror during its motion to make it work as a movable mirror in the miniaturized Michelson interferometer based FTIRs. To correct the tilt and ensure the resolution of the spectrum generated by the FTIRs, three conditions of the compensation system are proposed: 1) The reflected beam from the correcting mirror needs to vertically inject to the reflecting mirror; 2) The rotating axis of correcting mirror must be parallel to the tilting axis of translation mirror; and 3) The magnitude and direction of the corrected angle generated by correcting mirror needs to be same as the tilt angle of the translation mirror. A method is developed to determine the tilting axis of the translation mirror and a trial-and-error method is introduced to find out the current on the correcting mirror for compensation. In the experiment, an attractive force actuator introduced in [6], a 1D Lorenz force magnetic rotation mirror and a reflecting mirror compose the compensation system and its performance in a Michelson interferometer is tested. It proves that the designed compensation system can correct the tilt to $0.026^{\circ}$, so it is capable to measure substances with wavelength between $13.6 \mu \mathrm{m}$ and $25 \mu \mathrm{m}$, such as $\mathrm{SO}, \mathrm{PO}$ and $\mathrm{NO}$, which equals to half of the mid-infrared region. 


\section{CHAPTER 6 CONCLUSIONS AND FUTURE WORK}

\subsection{Conclusions and contributions}

An electromagnetic actuator based translation micromirror has been successfully developed in this thesis to be used in a miniaturized FTIRs. The cost and size of the micromirror is significantly reduced by compared with the movable mirror in the conventional FTIRs. The contributions of the thesis are in following three aspects:

(1) Developed a novel translation micromirror for the miniaturized FTIRs.

Two types of novel electromagnetic actuator based translation micromirrors, which have large displacement and high surface quality, are developed and presented in this thesis (Chapter $2 \& 4$ ). The actuators and mirror plates are fabricated separately using two processes and then bonded together. Therefore, the micromirrors can not only make large displacement of $123 \mu \mathrm{m}$ and $144 \mu \mathrm{m}$ by the attractive and repulsive magnetic force microactuator, respectively, but also have the high mirror surface quality, e.g., more than 15 meters curvature radius and $2 \mathrm{~nm}$ roughness. Due to the relative fast settling time, e.g., $16 \mathrm{~ms}$ downward and $50 \mathrm{~ms}$ upward for the attractive force actuator, and the capability to work in the quasi-static mode, they are more suitable to be used as the movable mirror in the FTIRs by compared with the electrothermal and electrostatic actuator based mircromirrors. Furthermore, the touching between the moving film and the substrate is completely eliminated by the repulsive force actuator before and in operation, therefore the starting position is constant each time and the repeatability is $<1 \%$. Because the micromirrors benefit from the MEMS fabrication technology, both their sizes and costs can be significantly reduced. For example, using the developed micromirror, the size of the movable mirror system can be reduced to around $100 \mathrm{~cm}^{3}$ from $4500 \mathrm{~cm}^{3}$ in the conventional FTIRs and the estimated cost can be reduced to $\$ 100$ from $\$ 10,000$.

(2) Developed a novel non-touching bonding technology for released microactuator.

In order to bond a high surface quality mirror plate with a released microactuator, a novel magnetic field pulling-force assisted bonding technology is developed and introduced in Chapter 3. This novel bonding technology utilizes non-touching magnetic field pulling-force, instead of the conventional compression mechanical force through touching, to bring the released microactuator and a mirror plate in contact for bonding such as to avoid: 1) any plastic deformation or damage to the released microactuator and 
2) the risk of sticking the actuator moving part to the substrate due to the adhesive squeezed out from the bonding surfaces. Furthermore, this bonding technology is not sensitive to the micromachining process, i.e., any process for microactuator fabrication containing the ferromagnetic structure layer can be used and bonded with a high surface quality mirror plate after released.

(3) Developed a tilt compensation system to correct the tilt during the motion of the translation micromirror.

Tilt exists not only in the micromirror but also in the conventional movable mirror in Michelson interferometer based FTIRs. Therefore, a novel tilt compensation method is proposed and presented in Chapter 5. This compensation system uses a correcting micromirror to correct the tilt of the translation micromirror and reflects the corrected beam to the beam splitter by a high surface quality flat mirror. This system is not only capable of compensating for the specific micromirrors, such as the developed micromirrors in this thesis, but also applicable to any translation mircomirror with a fixed tilting axis. Using current experiment setup and the fringe edge identification method, the tilt of the translation micromirror can be reduced to $0.026^{\circ}$ from $0.24^{\circ}$ after compensation, which makes the FTIRs able to measure substances in half of the midinfrared region, e.g., the wavelength between $13.6 \mu \mathrm{m}$ and $25 \mu \mathrm{m}$.

\subsection{Future work}

(1) Improve current compensation system

The experiment result in Chapter 5 shows the compensation system has the capacity to correct the tilt and can be used in the Michelson interferometer to measure infrared region above $13.6 \mu \mathrm{m}$. This result does not reflect the actual compensation capability of this system because of the measurement error and the equipment limitations. To further develop the potentiality of this compensation system, more precise results can be obtained through following: a) Use the repulsive force translation micromirror to replace the attractive force micromirror. As discussed in section 5.2.3, the starting position of the interference pattern varies $3 \sim 4 \mu$ m even after $20-30$ minutes pre-oscillation caused by the touching between the substrate and the moving film of the attractive force translation micromirror, so the reference pattern has to be measured for each independent 
experiment. In the repulsive force translation micromirror, there is no touching issue, so one reference pattern can be used to obtain a set of continuous measurements from the original position to the maximum displacement of the translation micromirror, which makes the current applied on the correcting mirror more accurate. In addition, the repeatability can also be improved by using the repulsive force actuator. However, challenges exist to use the repulsive force translation micromirror in the compensation system. A ring shaped permanent magnet is above the mirror plate, so the incident beam has to pass through the hole on the ring to reach the correcting mirror surface and it is same for the reflected beam from the mirror. Current ring shaped permanent magnet has a small inner diameter and it is rather far from the mirror plate, so the angle between the incident and the reflected beam is too small such that the reflected beam cannot reach the surface of the correcting mirror. Therefore, to overcome this problem, the structure of the repulsive magnetic force actuator, e.g. the shape of the permanent magnet and its distance to the mirror plate, needs to be re-designed so that the angle between the incident and the reflected beam becomes larger. b) Use a rotation micromirror with higher resolution to improve the compensation capability. When the resolution of the rotation mirror is higher, the fringe width after compensation can be closer to the reference width. c) Use the grayscale mapping tool on computers to identify the fringe edges automatically. As analyzed in section 5.3, the error in current compensation system is mainly caused by the inaccurate fringe width measurement on the screen using human eyes. If this process can be done using a computer assisted tool, the measurement result will be much more accurate.

(2) Perform the dynamic test of the response speed for the repulsive magnetic force actuator

The dynamic performance of the repulsive force micromirror needs to be tested, as the response speed tested for the attractive force micromirror in Chapter 2. Current main challenge for the dynamic test is caused by the distance between the ring shaped magnet and the mirror plate, which causes the angle between the incident beam and the reflected beam too small then the space above the actuator is not enough to accommodate the PSD and the laser source. To solve this problem, the solutions include: 1) Change the diameter of the ring shaped permanent magnet and re-design the assembly parts to reduce the 
distance between the ring shaped magnet and the mirror plate; 2) Use a unpackaged PSD and a smaller laser source so that they can be placed in the limited space above the micromirror. Once the challenge is resolved, the dynamic performance of the repulsive force micromirror can be obtained using the similar test method in Chapter 2 for the attractive force micromirror. 


\section{APPENDICES}

\section{Appendix A Simulation of the moving film deformation}

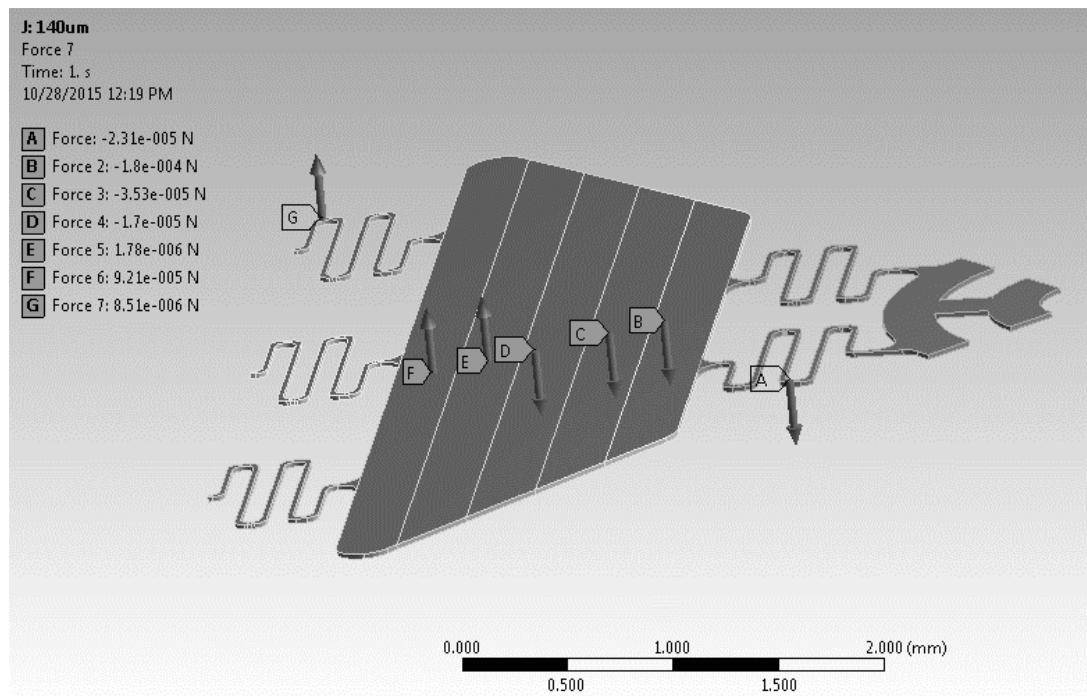

(a)

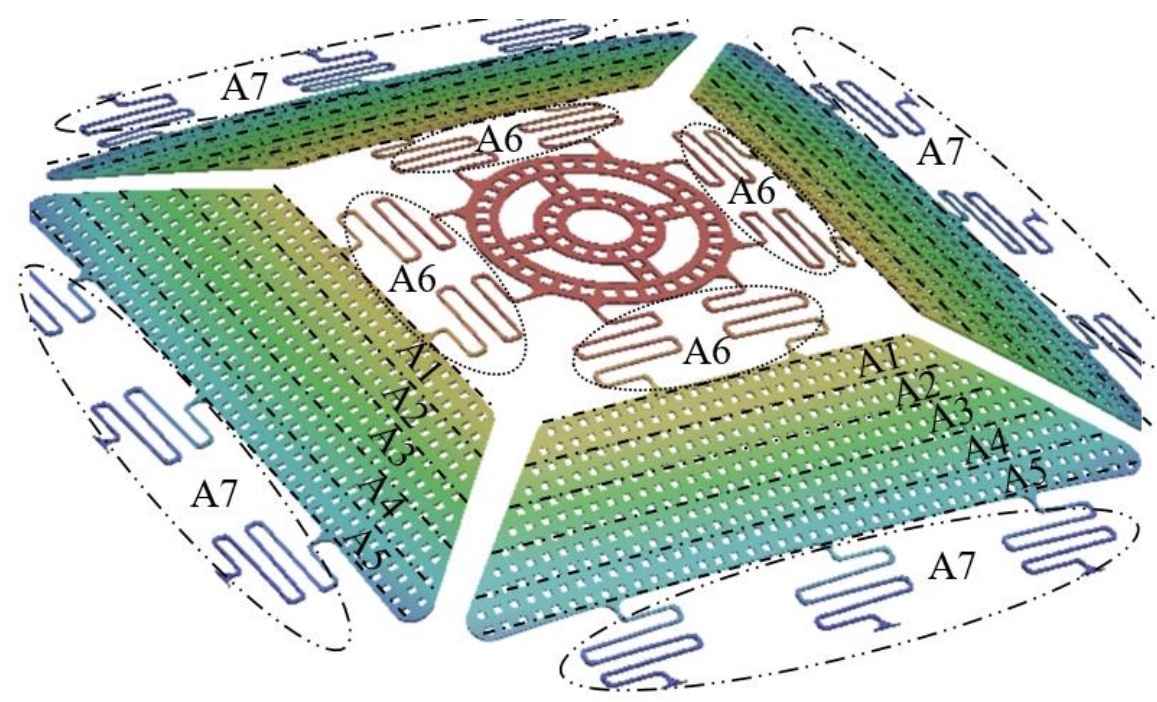

(b)

Figure A.1 Deformation of moving part simulated in "Static Structure" model. (a) Forces applied on 7 areas; (b) Deformation of moving part

The forces obtained on each element are exported to Excel and divided according to the 7 areas on a quarter of the moving film, as shown in Figure A.1a - the springs and the central ring structure are 2 areas, and the trapezoidal plate is equally divided into 5 areas along the height. As shown in Figure A.1b, the forces on the elements within the same 
area are summed up and a force is applied on the correspondent area in CoventorWare ${ }^{\circledR}$ MemMech module to simulate the deformation of the moving film, which is caused not only by the magnetic force, but also by the residual stress gradient and the weight of the mirror plate. Finally, the central ring is raised to $368 \mu \mathrm{m}$ under the magnetic force generated by the ring shape permanent magnet without applying a current on the electromagnet.

\section{Appendix B Moving film moves downward when the electromagnet has a small core}

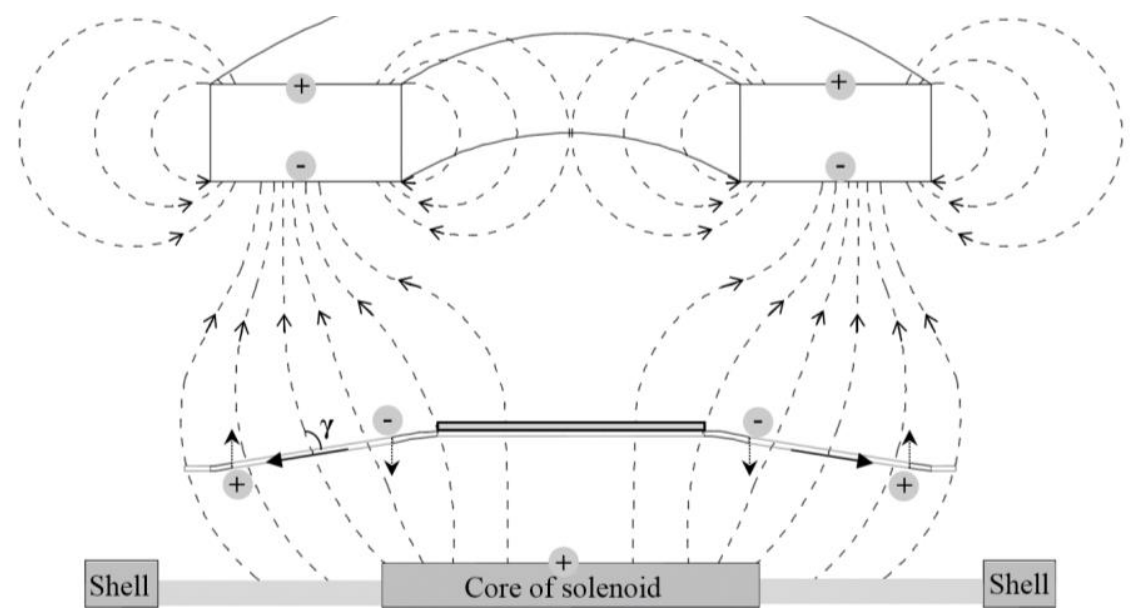

(a)

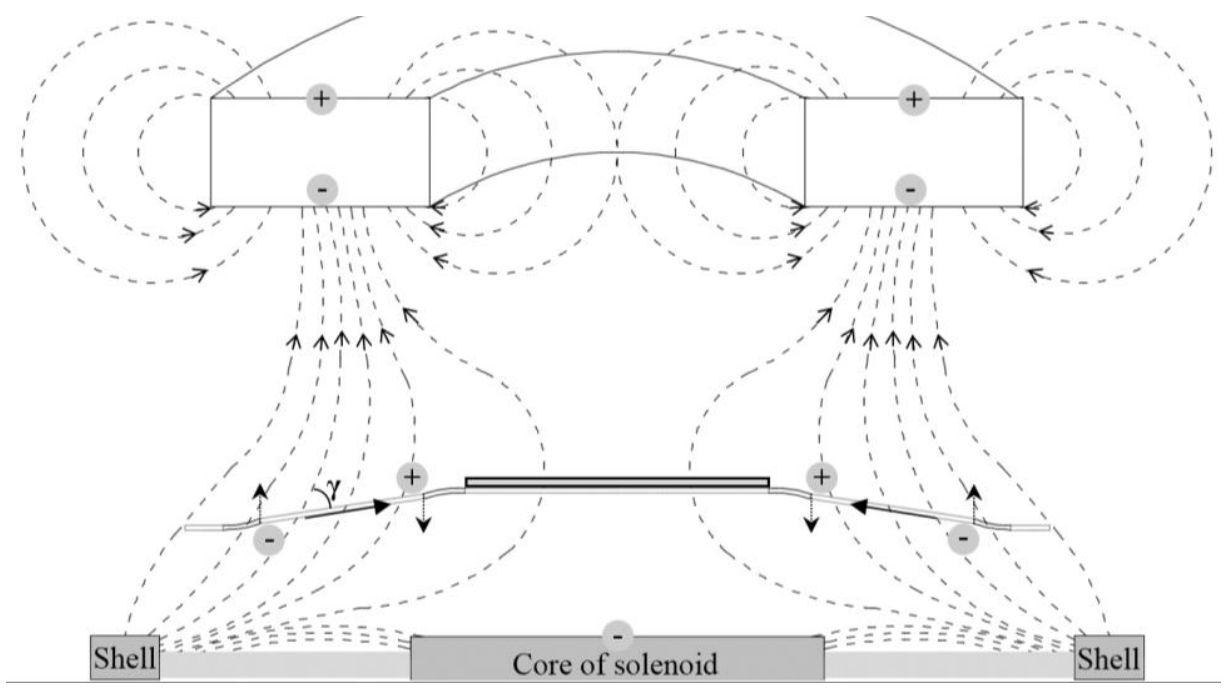

(b)

Figure A.2 Moving part moves downward when the electromagnet core is smaller than the moving part. (a) North pole formed on the core top surface; (b) South pole formed on the core top surface. 
An electromagnet with the small core leads to the trapezoidal plate be attracted down towards the core, no matter what the direction of the current is. For example, when the current is applied counterclockwise as shown in Figure A.2a, the direction of the in-plane magnetization is directed to the lower edge of the plate because the angle $\gamma$ between the external magnetic field $H$ and the in-plane magnetization $M$ is larger than $90^{\circ}$. As the result, the magnetic pole on the upper edge of the plate is switched to south which is attracted towards the core due to the different magnetic pole on the top surface of the core. In contrast, a clockwise current applied on the electromagnet leads to a magnetic south pole be formed on the top surface of the core and $\gamma$ is smaller than $90^{\circ}$, as shown in Figure A.2b. In this case, the in-plane magnetization is directed to the upper edge, where the magnetic north pole is formed. As the result, the moving film moves downward to the electromagnet due to the different magnetic poles between them.

\section{Appendix C PSD based displacement measurement}

A displacement measurement process and the setup are developed as illustrated in Figure A.3. The mirror plate of the micromirror is on the $\mathrm{X}-\mathrm{Y}$ plane where the $\mathrm{X}$-axis is parallel to the table (X-Z plane) and the $\mathrm{Y}$-axis is perpendicular to it. A laser beam parallel to the table is injected to the mirror plate and a PSD detects the trajectory of the reflected beam. The output of the PSD is connected to a digital phosphor oscilloscope, which shows the voltage change of the output on both $\mathrm{X}$ and $\mathrm{Y}$ axis. Basing on the display on the oscilloscope, the $\mathrm{x}$ and y positions of the reflected dot on the PSD can be determined. By comparing the measured moving distance of the reflected dot on the PSD with the calibration data, the translation of the micromirror when driving the actuator can be calculated. The calibration data is obtained by recording the moving distance of the reflected laser dot on the PSD when manually translating the micromirror, which sits on a fine translation stage with moving direction perpendicular to the mirror plate surface (along Z-axis).

During a pure translation, the mirror moves along the Z-axis and the trajectory on the PSD is always parallel to the table (X-axis), as shown in Figure A.3b. Once the micromirror translates with the tilt, the trajectory is in an angle with the $\mathrm{X}$-axis as illustrated in Figure A.3c unless the rotation axis is parallel to Y. In order to obtain the 
actual translation displacement of the mirror, the following steps are performed to distinguish the translation from the rotation.

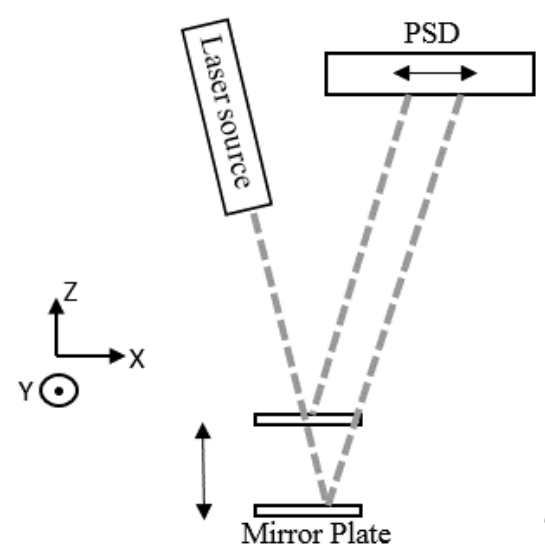

(a)

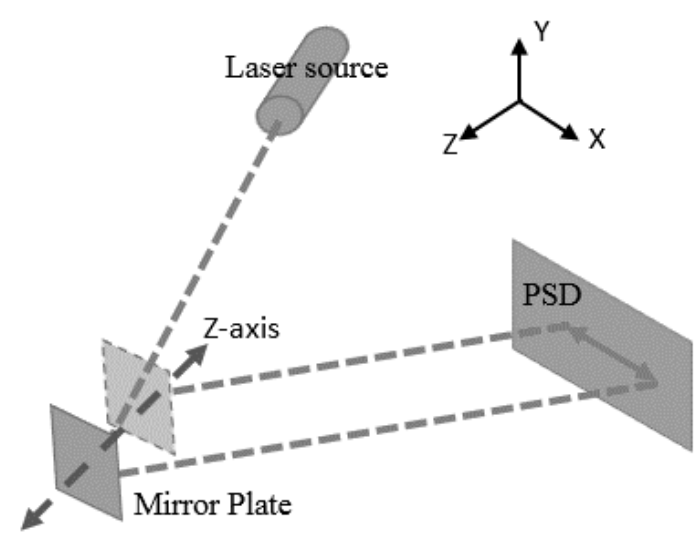

(b)

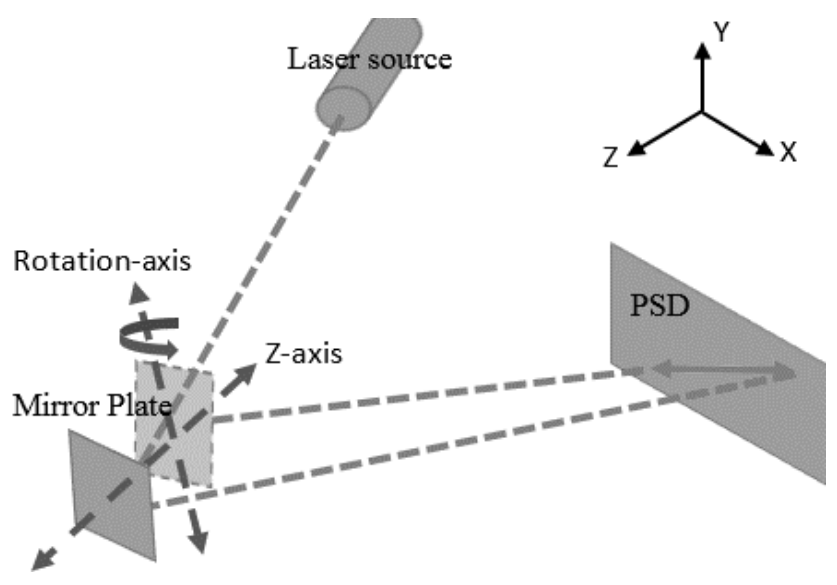

(c)

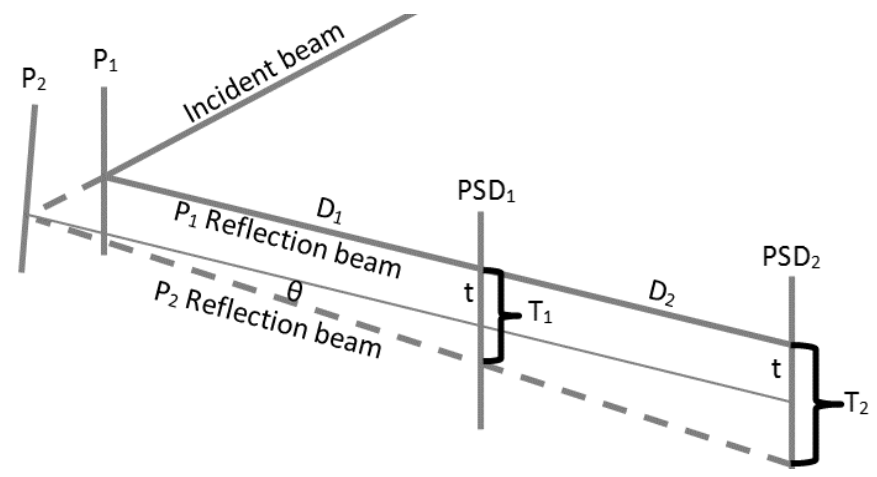

(d)

Figure A.3 Concept of the displacement measurement. (a) Micromirror translates along the Z-axis (2D); (b) Micromirror translates along the Z-axis (3D); (c) Micromirror in an angle with the X-axis (3D); (d) 2D schematic diagram of accurate examination platform. 
1. Rotating the mircomirror around the Z-axis until the trajectory of the reflection beam is parallel to the $\mathrm{X}$-axis. Now the rotating axis is perpendicular to the table and parallel to the Y-axis. The displacements caused by both translation and rotation are on the same line (X-axis).

2. Rotating the micromirror $90^{\circ}$ around the $\mathrm{Z}$-axis, then its rotation axis is parallel to the table (X-axis). At this time, the translational displacement is still parallel to the $\mathrm{X}$ axis while the displacement caused by rotation is turned to $\mathrm{Y}$ direction. So the translation and the rotation are distinguished in $\mathrm{X}$-axis and $\mathrm{Y}$-axis, respectively. Using the above method, the translation displacement can be found. However, it is quite possible that the laser dot is not injected on the rotation axis of the mirror. In this case, the length of the trajectory of the reflected beam on the PSD varies with the location of the incident point on the mirror. To avoid the error caused by it, the micromirror is rotated $180^{\circ}$ after the translation displacement is found at step 2. A second translation displacement can be derived following the same method. By taking a median of these 2 displacements, the error caused by the incident point is eliminated and then the actual translation displacement can be obtained.

Using above method, an approximate displacement and the tilting angle can be obtained. To further reduce the errors and get the accurate displacement and the tilting angle, the PSD is moved along a straight line as shown in Figure A.3d. When the micromirror is located at any position between $\mathrm{P} 1$ and $\mathrm{P} 2$, the trajectory of the reflected beam on PSD should be identical if it makes pure transition. When the mirror rotates with an angle $\theta$, the trajectory of the reflected beam varies when the mirror's position changes. In this situation, the translation displacement and the tilting angle can be obtained using the equations below:

$$
\begin{gathered}
T_{1}=D_{1} \tan \theta+\mathrm{t} \\
T_{2}=\left(D_{1}+D_{2}\right) \tan \theta+\mathrm{t}
\end{gathered}
$$

Similarly, to avoid the errors caused by the position of the incident beam, the values are measured and calculated again after the micromirror is rotated $180^{\circ}$ and the equations are: 


$$
\begin{gathered}
T_{3}=D_{1} \tan (-\theta)+\mathrm{t} \\
T_{4}=\left(D_{1}+D_{2}\right) \tan (-\theta)+\mathrm{t}
\end{gathered}
$$

where $T_{x}$ is the trajectory of the reflected beam on the PSD, $D_{x}$ is the distance between the micromirror and the PSD $\left(D_{x} \gg T_{x}\right), \theta$ is the tilting angle, and $t$ is the displacement caused by pure translation. 


\section{REFERENCES}

[1] Stephen A. Dyer, Wiley Survey of Instrumentation and Measurement. .

[2] Paul M Robertson, "What is a grating," 2014. [Online]. Available: http://hpf.psu.edu/2014/03/19/the-echelle-grating/.

[3] S. J. Anne Thorne, Ulf Litzén, "Spectrophysics: Principles and Applications," Springer Science \& Business Media, 1999.

[4] K. Citek, UV and Blue-Violet Light Definitions, risks and prevention, Special Ed. international review of ophthalmic optics, 2016.

[5] Linda J. Vandergriff, "Nature and Properties of Light," in Fundamentals of Photonics, Wiley, pp. 19-20.

[6] Ramesh Raghavachari, Near-Infrared Applications in Biotechnology. Marcel Dekker, Inc., 2001.

[7] Nancy Birkner, Qian Wang, "How an FTIR Spectrometer Operates," Chemistry Libretests, 2015. [Online]. Available: https://chem.libretexts.org/.

[8] R. B. Gustav Kirchhoff, "Chemical Analysis by Observation of Spectra," Annalen der Physik und der Chemie (Poggendorff), 1860. [Online]. Available: http://www.chemteam.info/Chem-History/Kirchhoff-Bunsen-1860.html.

[9] D. Harvey, “Atomic Absorption Spectroscopy,” Chemistry Libretests. [Online]. Available: https://chem.libretexts.org/.

[10] P. Elmer, "New Coleman Junior II Spectrophotometer," Anal. Chem., vol. 41, no. 3, p. 57A-57A, 1969.

[11] B. C. Smith, Fundamentals of Fourier transform infrared spectroscopy. 2011.

[12] L. D. Spencer, "Spectral characterization of the herschel SPIRE Photometer," University of Lethbridge Lethbridge, 2005.

[13] SHIMADZU, "60 years Infrared Spectrophotometers," SHIMADZU EUROPA GMBH ANALYTICAL AND MEASURING INSTRUMENTS. [Online]. Available: https://www.shimadzu.eu/60-years-infrared-spectrophotometers.

[14] Mike Morris, “Ocean Optics Spectrometers.” [Online]. Available: http://www.spectrecology.com/.

[15] Ocean Optics Inc., "S2000 Miniature Fiber Optic Spectrometers and Accessories 
Document." [Online]. Available: https://neurophysics.ucsd.edu/Manuals/Ocean Optics/S2000_Operating_Manual.pdf.

[16] Ocean Optics, “USB 2000+ UV-VIS.” [Online]. Available:

https://oceanoptics.com/product/usb2000uv-vis/.

[17] C. Solf, J. Mohr, and U. Wallrabe, "Miniaturized LIGA Fourier transformation spectrometer," Sensors, 2003. Proc., pp. 773-776, 2003.

[18] “Nicolet AVATAR 360 FTIR,” SpectraLab Scientific Incorporation. [Online]. Available: http://www.spectralabsci.com/Products/Default.aspx?productId=1081.

[19] Danielle Collins, “Air bearing basics," Linear Motion Tips, 2015. [Online]. Available: http://www.linearmotiontips.com/air-bearing-basics/.

[20] “Noppon Bearing SEBS 5B,” Noppon Bearing. [Online]. Available: https://www.nbcorporation.com/index.html.

[21] "PI Air bearing linear stages," PI motion and Position. [Online]. Available: http://www.pi-usa.us/products/Air_Bearing_Stages/index.php.

[22] R. Nesbitt, "Holographic recording using a digital micromirror device," Proc. SPIE 3637, Pract. Hologr. XIII, 12, vol. 3637, no. January, pp. 12-20, 1999.

[23] A. D. Yalcinkaya, H. Urey, D. Brown, T. Montague, and R. Sprague, "Two-axis electromagnetic microscanner for high resolution displays," $J$. Microelectromechanical Syst., vol. 15, no. 4, pp. 786-794, 2006.

[24] A. Jain and H. Xie, "A single-crystal silicon micromirror for large bi-directional 2D scanning applications," Sensors Actuators, A Phys., vol. 130-131, no. SPEC. ISS., pp. 454-460, 2006.

[25] C. Fan and S. He, "A microelectrostatic repulsive-torque rotation actuator with two-width fingers," J. Micromechanics Microengineering, vol. 25, no. 9, p. 95006, 2015.

[26] S. He and R. Ben Mrad, "Large-stroke microelectrostatic actuators for vertical translation of micromirrors used in adaptive optics," IEEE Trans. Ind. Electron., vol. 52, no. 4, pp. 974-983, 2005.

[27] S. He and R. Ben Mrad, "Design, modeling, and demonstration of a MEMS repulsive-force out-of-plane electrostatic micro actuator," $J$.

Microelectromechanical Syst., vol. 17, no. 3, pp. 532-547, 2008. 
[28] S. He and J. S. Chang, "Experimental verification of an out-of-plane repulsiveforce electrostatic actuator using a macroscopic mechanism," Microsyst. Technol., vol. 15, no. 3, pp. 453-461, 2009.

[29] J. Chong, S. He, and R. Ben Mrad, "Development of a vector display system based on a surface-micromachined micromirror," IEEE Trans. Ind. Electron., vol. 59, no. 12, pp. 4863-4870, 2012.

[30] S. He, R. Ben Mrad, and J. Chong, "Repulsive-force out-of-plane large stroke translation micro electrostatic actuator," J. Micromechanics Microengineering, vol. 21, no. 7, p. 75002, 2011.

[31] C. Fan, S. He, and S. Member, "A Two-Row Interdigitating-Finger RepulsiveTorque Electrostatic Actuator and Its Application to Micromirror Vector Display," J. Microelectromechanical Syst., vol. 24, no. 6, pp. 1-13, 2015.

[32] F. Chao, S. He, J. Chong, R. Ben Mrad, and L. Feng, "Development of a micromirror based laser vector scanning automotive HUD," 2011 IEEE Int. Conf. Mechatronics Autom. ICMA 2011, pp. 75-79, 2011.

[33] S. He, R. Ben Mrad, and J. S. Chang, "Development of a High-Performance Microelectrostatic Repulsive-Force Rotation Actuator," J. Microelectromechanical Syst., vol. 19, no. 3, pp. 561-569, 2010.

[34] M. C. Wu, O. Solgaard, and J. E. Ford, "Optical MEMS for lightwave communication," J. Light. Technol., vol. 24, no. 12, pp. 4433-4454, 2006.

[35] A. Debray, A. Ludwig, T. Bourouina, A. Asaoka, N. Tiercelin, G. Reyne, T. Oki, E. Quandt, H. Muro, and H. Fujita, "Application of a multilayered magnetostrictive film to a micromachined 2-D optical scanner," $J$. Microelectromechanical Syst., vol. 13, no. 2, pp. 264-271, 2004.

[36] H. Miyajima, N. Asaoka, M. Arima, Y. Minamoto, K. Murakami, K. Tokuda, and K. Matsumoto, "A durable, shock-resistant electromagnetic optical scanner with polyimide-based hinges," J. Microelectromechanical Syst., vol. 10, no. 3, pp. 418424, 2001.

[37] H. Toshiyoshi, W. Piyawattanametha, C. T. Chan, and M. C. Wu, "Linearization of electrostatically actuated surface micromachined 2-D optical scanner," $J$. Microelectromechanical Syst., vol. 10, no. 2, pp. 205-214, 2001. 
[38] T. Bifano, "Adaptive imaging: MEMS deformable mirrors," Nat. Photonics, vol. 5, no. 1, pp. 21-23, 2011.

[39] J. Liang, B. Grimm, S. Goelz, and J. F. Bille, "Objective measurement of the wave aberration of human eye with the use of a Hartmann-Shack wave-front sensor," $J$. Opt. Soc. Amer. A, Opt. Image Sci., vol. 11, pp. 1949-1957, 1994.

[40] N. Doble and D.R. Williams, "The Application of MEMS Technology for Adaptive Optics in Vision Science," IEEE J. Sel. Top. QUANTUM Electron., vol. 10, pp. 625-629, 2004.

[41] J. Liang, D. R. Williams, and D. T. Miller. "Supernormal vision and highresolution retinal imaging through adaptive optics," J. Opt. Soc. Amer. A, Opt. Image Sci, vol. 14, pp. 2884-2892, 1997.

[42] R. J. Zawadzki, S. M. Jones, S. S. Olivier, M. T. Zhao, B. A. Bower, J. A. Izatt, S. Choi, S. Laut, and J. S. Werner. "Adaptive-optics optical coherence tomography for high-resolution and high-speed 3D retinal in vivo imaging," Opt. Express, vol. 13, pp. 4792-4811, 2005.

[43] A. W. Dreher, J. F. Bille, and R. N. Weinreb, "Active optical depth resolution improvement of the laser tomographic scanner," Appl. Opt., vol. 28, pp. 804-808, 1989.

[44] J. Liang, D. R. Williams, and D. T. Miller., "Aberrations and retinal image quality of the normal human eye," Opt. Soc. Amer. A, Opt. Image Sci., vol. 14, pp. 28732883, 1997.

[45] T. Sandner, T. Grasshoff, E. Gaumont, H. Schenk, and A. Kenda, "Translatory MOEMS actuator and system integration for miniaturized Fourier transform spectrometers," J. Micro/Nanolithography, MEMS, MOEMS, vol. 13, no. 1, p. 11115, 2014.

[46] J. S. Park, L. L. Chu, A. D. Oliver, and Y. B. Gianchandani, "Bent-beam electrothermal actuators-Part II: Linear and rotary microengines," $J$. Microelectromechanical Syst., vol. 10, no. 2, pp. 255-262, 2001.

[47] W. Wang, J. Chen, A. S. Zivkovic, C. Duan, and H. Xie, “A silicon based fourier transform spectrometer base on an open-loop controlled electrothermal mems mirror," in IEEE Transducers 2015, 2015, pp. 212-215. 
[48] A. Kenda, C. Drabe, H. Schenk, A. Frank, M. Lenzhofer, and W. Scherf, "Application of a Micromachined Translatory Actuator to an optical FTIR Spectrometer," in SPIE, MEMS, MOEMS, and Micromachining II, 2006, vol. 6186, pp. 1-11.

[49] C. Ataman, H. Urey and A. Wolter, "A Fourier transform spectrometer using resonant vertical comb actuators," J. Micromechanics Microengineering, vol. 16, no. $12,2006$.

[50] J. M. D. and L. F. Leo P Schuler, Jason S Milne, "MEMS-based microspectrometer technologies for NIR and MIR wavelengths," J. Phys. D. Appl. Phys., vol. 42, no. 13, 2009.

[51] W. Wang, J. Chen, A. S. Zivkovic, Q. A. A. Tanguy, and H. Xie, "A Compact Fourier Transform Spectrometer on a Silicon Optical Bench With an Electrothermal MEMS Mirror," J. Microelectromechanical Syst., vol. 25, no. 2, pp. 347-355, 2016.

[52] P. Microsystems and C. Tech, "Out-of-plane translatory MEMS actuator with extraordinary large stroke for optical path length modulation in miniaturized FTIR spectrometers," in Sensor and Test, 2011, pp. 151-156.

[53] H. Xie, S. Lan, D. Wang, W. Wang, J. Sun, H. Liu, J. Cheng, J. Ding, Z. Qin, Q. Chen, H. Kang, and Z. Tian, "Miniature Fourier transform spectrometers based on electrothermal MEMS mirrors with large piston scan range," in IEEE, 2015, pp. 36.

[54] B. Saadany, H. Omran, M. Medhat, F. Marty, D. Khalil, and T. Bourouina, "MEMS tunable Michelson interferometer with robust beam splitting architecture," 2009 IEEE/LEOS Int. Conf. Opt. MEMS Nanophotonics, OPTMEMS 2009, pp. 49-50, 2009.

[55] B. Engineering, "Michelson Interferometer Operation," Block Engineering, Inc. All rights reserved. [Online]. Available: http://www.blockeng.com/technology/ftirtechnology.html.

[56] Newport, "Introduction to FTIR Spectroscopy," Newport Corporation. All rights reserved, 2016. [Online]. Available: http://www.newport.com/Introduction-toFTIR-Spectroscopy/405840/1033/content.aspx. 
[57] S. H. and R. B. M. Zewdu Hailu, "A novel vertical comb-drive electrostatic actuator using a one layer process," J. Micromechanics Microengineering, vol. 24, no. 11, 2014.

[58] S. H. Sadat, D. Kamiya, and M. Horie, "Large-Deflection Spiral-Shaped Micromirror Actuator," J. Microelectromechanical Syst., vol. 18, no. 6, pp. 13571364, 2009.

[59] J. D. Grade and H. Jerman, "MEMS electrostatic actuators for optical switching applications," OFC 2001. Opt. Fiber Commun. Conf. Exhib. Tech. Dig. Postconf. Ed. (IEEE Cat. 01CH37171), vol. 3, p. WX2/1-WX2/3, 2001.

[60] W. Zhang, R. Baskaran, and K. L. Turner, "Effect of cubic nonlinearity on autoparametrically amplified resonant MEMS mass sensor," Sensors Actuators A Phys., vol. 102, no. 1-2, pp. 139-150, 2002.

[61] Texas Instruments, "DMD 101 : Introduction to Digital Micromirror Device ( DMD ),” no. October, pp. 1-11, 2013.

[62] C. Liao and J. Tsai, "The Evolution of MEMS Displays," IEEE Trans. Ind. Electron., vol. 56, no. 4, pp. 1057-1065, 2009.

[63] C. Drabe, T. Klose, H. Schenk, A. Wolter, and H. Lakner, "A large deflection translatory actuator for optical path length modulation," in SPIE, MEMS, MOEMS, and Micromachining II, 2006, vol. 6186, pp. 618604-618604-8.

[64] D. S. Greywall, P. A. Busch, F. Pardo, "Crystalline silicon tilting mirrors for optical cross-connect switches. Microelectromechanical System," Microelectromechanical Syst. J. of, vol. 12, no. 5, pp. p708-712, 2003.

[65] C. Liu, Foundations of MEMS, Second Edition, 2nd ed. 2011.

[66] K. Yu, D. Lee, and U. Krishnamo, "Micromachined Fourier Transform spectrometer on silicon optical bench platform," in IEEE Actuators and Microsystems, 2005, pp. 1250-1254.

[67] O. Manzardo, H. P. Herzig, C. R. Marxer, and N. F, "Miniaturized time-scanning Fourier transform spectrometer based on silicon technology," in Opt. Lett., 1999, vol. 24, no. 23, pp. 1705-1707.

[68] T. Sandner, A. Kenda, C. Drabe, H. Schenk, and W. Scherf, "Miniaturized FTIRspectrometer based on optical MEMS translatory actuator," Proc. SPIE, vol. 6466, 
pp. 646602-646602-12, 2007.

[69] H. R. Seren, N. P. Ayerden, J. Sharma, S. T. S. Holmström, T. Sandner, T. Grasshoff, H. Schenk, and H. Urey, "Lamellar grating based MEMS Fourier Transform Spectrometer," Opt. MEMS Nanophotonics (OPT MEMS), 2010 Int. Conf., pp. 105-106, 2010.

[70] F. Han, W. Wang, X. Zhang, H. Xie, S. Member, and A. Abstract, "Modeling and Control of a Large-Stroke Electrothermal MEMS Mirror for Fourier Transform Microspectrometers," J. Microelectromechanical Syst., vol. 25, no. 4, pp. 750760, 2016.

[71] A. Werber and H. Zappe, "Thermo-pneumatically actuated, membrane-based micro-mirror devices," J. Micromechanics Microengineering, vol. 16, no. 12, pp. 2524-2531, 2006.

[72] A. Werber and H. Zappe, "A thermo-pneumatically actuated tip-tilt-piston mirror," in TRANSDUCERS and EUROSENSORS '07 - 4th International Conference on Solid-State Sensors, Actuators and Microsystems, 2007, pp. 1525-1528.

[73] A. Jain, H. Qu, S. Todd, and H. Xie, "A thermal bimorph micromirror with large bi-directional and vertical actuation," Sensors Actuators A Phys., vol. 122, no. 1, pp. 9-15, 2005.

[74] S. R. Samuelson and H. Xie, "A large piston displacement MEMS mirror with electrothermal ladder actuator arrays for ultra-low tilt applications," $J$. Microelectromechanical Syst., vol. 23, no. 1, pp. 39-49, 2014.

[75] X. Zhang, L. Zhou, and H. Xie, "A Fast, Large-Stroke Electrothermal MEMS Mirror Based on Cu/W Bimorph," Micromachines, vol. 6, no. 12, pp. 1876-1889, 2015.

[76] L. Wu and H. Xie, "A large vertical displacement electrothermal bimorph microactuator with very small lateral shift," Sensors Actuators, A Phys., vol. 145146, no. 1-2, pp. 371-379, 2008.

[77] L. Wu, a. Pais, S. R. Samuelson, S. Guo, and H. Xie, "A mirror-tilt-insensitive fourier transform spectrometer based on a large vertical displacement micromirror with dual reflective surface," TRANSDUCERS 2009 - 15th Int. Conf. Solid-State Sensors, Actuators Microsystems, pp. 2090-2093, 2009. 
[78] Ç. Ataman and H. Urey, "Compact Fourier transform spectrometers using FR4 platform," Sensors Actuators A Phys., vol. 151, no. 1, pp. 9-16, 2009.

[79] M. Stifter, H. Steiner, A. Kainz, F. Keplinger, W. Hortschitz, and T. Sauter, "A Lorentz force actuated magnetic field sensor with capacitive read-out," Conf. Smart Sensors, Actuators, MEMS VI, April 24, 2013 - April 26, 2013, vol. 8763, p. The Society of Photo-Optical Instrumentation Engin, 2013.

[80] L. Chen, Z. Z. Wen, R. Qian, C. Li, Zhi-yu Wen, Zhong-quan Wen, and Q. Rongrong, "A novel electromagnetically actuated MEMS scanning mirror integrated with rotation angle sensor," Proc. SPIE, vol. 8191, pp. 819125-819125-8, 2011.

[81] M. Li, V. T. Rouf, G. Jaramillo, and D. A. Horsley, "MEMS Lorentz force magnetic sensor based on a balanced torsional resonator," 2013 17th Int. Conf. Solid-State Sensors, Actuators Microsystems, TRANSDUCERS EUROSENSORS 2013, June 16, 2013 - June 20, 2013, no. June, pp. 66-69, 2013.

[82] H. Urey, S. Holmstrom, and A. D. Yalcinkaya, "Electromagnetically Actuated FR4 Scanners," IEEE Photonics Technol. Lett., vol. 20, no. 1, pp. 30-32, 2008.

[83] M. Feldmann, A. Waldschik, and S. Büttgenbach, "Electromagnetic microactuators, micro-motors, and micro-robots," in SPIE, Microelectronics: Design, Technology, and Packaging III, 2007, vol. 6798, pp. 1-10.

[84] A. Cao, J. Kim, and L. Lin, "Bi-directional electrothermal electromagnetic actuators," J. Micromechanics Microengineering, vol. 17, no. 5, pp. 975-982, 2007.

[85] M. Feldmann and S. Büttgenbach, "Novel microrobots and micromotors using lorentz force driven linear microactuators based on polymer magnets," IEEE Trans. Magn., vol. 43, no. 10, pp. 3891-3895, 2007.

[86] S. Forouzanfar, R. Mansour, and E. Abdel-Rahman, "Lorentz-force transduction for RF micromechanical filters," J. Micromechanics Microengineering, vol. 22, no. 3, p. 35018, 2012.

[87] J. J. Bernstein, W. P. Taylor, J. D. Brazzle, C. J. Corcoran, G. Kirkos, J. E. Odhner, A. Pareek, M. Waelti, and M. Zai, "Electromagnetically actuated mirror arrays for use in 3-D optical switching applications," J. Microelectromechanical Syst., vol. 13, no. 3, pp. 526-535, 2004. 
[88] G. Brown, R. Bauer, W. Lubeigt, and D. Uttamchandani, "SOI based electromagnetic MEMS scanners and their applications in laser systems," SPIE, MOEMS Miniaturized Syst. XII, vol. 8616, pp. 1-9, 2013.

[89] I. J. Cho and E. Yoon, “A low-voltage three-axis electromagnetically actuated micromirror for fine alignment among optical devices," J. Micromechanics Microengineering, vol. 19, no. 8, p. 85007, 2009.

[90] Y. Xue and S. He, "A translation micromirror with large quasi-static displacement and high surface quality," J. Micromechanics Microengineering, vol. 27, no. 1, pp. $1-10,2016$.

[91] K. Riaz, S. A. Bazaz, M. M. Saleem, and R. I. Shakoor, "Design, damping estimation and experimental characterization of decoupled 3-DoF robust MEMS gyroscope," Sensors Actuators A Phys., vol. 172, no. 2, pp. 523-532, 2011.

[92] S. O. Isikman and H. Urey, "Dynamic Modeling of Magnetic Film Actuators," LEOS 2007 - IEEE Lasers Electro-Optics Soc. Annu. Meet. Conf. Proc., no. 1, pp. 912-913, 2007.

[93] H. Toshiyoshi, D. Miyauchi, and H. Fujita, "Electromagnetic torsion mirrors for self-aligned fiber-optic crossconnectors by silicon micromachining," Sel. Top. Quantum Electron. IEEE J., vol. 5, no. 1, pp. 10-17, 1999.

[94] Ç. Ataman, S. Lani, W. Noell, and N. de Rooij, “A dual-axis pointing mirror with moving-magnet actuation," J. Micromechanics Microengineering, vol. 23, no. 2, p. $25002,2013$.

[95] P. H. Cu-Nguyen, A. Grewe, C. Endrödy, S. Sinzinger, H. Zappe, and A. Seifert, “Compact tunable hyperspectral imaging system," Proc. IEEE Int. Conf. Micro Electro Mech. Syst., pp. 1167-1170, 2014.

[96] J. Suzuki, T. Miura, K. Terao, H. Takao, F. Shimokawa, T. Namazu, F. Oohira, and T. Suzuki, "Dual-axis Polymer-MEMS Mirror Made of Photosensitive Nanocomposite," in Optical MEMS and Nanophotonics 2013, Kanazawa, Japan, 18-22 August 2013, 2013, no. August, pp. 101-102.

[97] J. Streque, A. Talbi, C. Bonnerot, P. Pernod, and V. Preobrazhensky, "Magnetostatic micro-actuator based on ultrasoft elastomeric membrane and copper - Permalloy electrodeposited structures," 2012 IEEE 25th Int. Conf. 
Micro Electro Mech. Syst., no. February, pp. 1157-1160, 2012.

[98] N. Weber, H. Zappe, and A. Seifert, “An all-nickel magnetostatic MEMS scanner," J. Micromechanics Microengineering, vol. 22, no. 12, pp. 125008$125015,2012$.

[99] S. O. Isikman and H. Urey, "Dynamic modeling of soft magnetic film actuated scanners," IEEE Trans. Magn., vol. 45, no. 7, pp. 2912-2919, 2009.

[100] A. M. Morega, S. Member, N. T, M. Popa, M. Morega, and J. B. Dumitru, "Numerical Simulation of an Electromagnetic Bending-Mode Cantilever Microactuator," in The 8th International Symposium on Advanced Topics in Electrical Engineering, 2013, no. 13, pp. 1-6.

[101] A. D. Yalcinkaya, O. Ergeneman, and H. Urey, "Polymer magnetic scanners for bar code applications," Sensors Actuators A Phys., vol. 135, no. 1, pp. 236-243, 2007.

[102] J. W. Judy, R. S. Muller, and H. H. Zappe, "Magnetic microactuation of polysilicon flexure structures," J. Microelectromechanical Syst., vol. 4, no. 4, pp. 162-169, 1995.

[103] D. J. Sadler, T. M. Liakopoulos, and C. H. Ahn, "Universal electromagnetic microactuator using magnetic interconnection concepts," $J$. Microelectromechanical Syst., vol. 9, no. 4, pp. 460-468, 2000.

[104] B. Wagner, M. Kreutzer, and W. Benecke, "Electromagnetic microactuators with multiple degrees of freedom," in IEEE, 1991, pp. 614-617.

[105] M. Wang, Y. Zhang, G. P. Li, and M. Bachman, "Resonance-based addressing in laminate MEMS devices," 2012 IEEE 62nd Electron. Components Technol. Conf., pp. 2048-2052, 2012.

[106] A. Grewe, S. Sinzinger, H. Zappe, and A. Seifert, “Tunable confocal hyperspectral imaging system," in Optical MEMS and Nanophotonics 2013, Kanazawa, Japan, 18-22 August 2013 Tunable, 2013, vol. 2, no. August, pp. 9-10.

[107] Y. Kim, N. G. Kim, J. M. Kim, S. H. Lee, Y. Kwon, and Y. K. Kim, “60-GHz full MEMS antenna platform mechanically driven by magnetic actuator," IEEE Trans. Ind. Electron., vol. 58, no. 10, pp. 4830-4836, 2011.

[108] G. D. Gray and P. A. Kohl, "Modeling and performance of a magnetic MEMS 
wiping actuator," Microelectromechanical Syst. J., vol. 15, no. 4, pp. 904-911, 2006.

[109] V. Badilita1, K. Kratt1, T. Burger, J.G. Korvink, and U. Wallrabe1, “3D High Aspect Ratio, Mems Integrated Micro-Solenoids and Helmholtz Micro-Coils," Solid-State Sensors, Actuators Microsystems Conf. 2009. TRANSDUCERS 2009. Int., pp. 1106-1109, 2009.

[110] B. Liu, D. Li, X. Yang, and X. Li, "Design and fabrication of a micro electromagnetic actuator," Proc. 1st IEEE Int. Conf. Nano Micro Eng. Mol. Syst. 1st IEEE-NEMS, pp. 353-356, 2006.

[111] H. J. Cho and C. H. Ahn, "A Bidirectional Magnetic Microactuator Using Electroplated Permanent Magnet Arrays," J. Microelectromechanical Syst., vol. 11, no. 1, pp. 78-84, 2002.

[112] T. Nakahara, Y. Hosokawa, K. Terao, H. Takao, and F. Shimokawa, "Self-Aligned Fabrication Process for Active Membrane Made of Photosensitive Nanocomposite," in MEMS 2012, Paris, FRANCE, 29 January - 2 February 2012, 2012, no. February, pp. 1181-1184.

[113] B. Assadsangabi, M. S. M. Ali, and K. Takahata, "Planar variable inductor controlled by ferrofluid actuation," IEEE Trans. Magn., vol. 49, no. 4, pp. 14021406, 2013.

[114] M. K. Yapici, J. M. Hong, J. Zou, and K. Balareddy, "Post-CMOS on-chip integration of 3D MEMS inductors using a novel chip embedding technique," 2013 Transducers Eurosensors XXVII 17th Int. Conf. Solid-State Sensors, Actuators Microsystems, TRANSDUCERS EUROSENSORS 2013, no. June, pp. 128-131, 2013.

[115] M. Yoichi, “Applications of Piezoelectroin Actuator, " NEC technical Journal, vol. 1 , no. 5 , pp. $82-86$

[116] “P-088 Round PICMA® Stack Multilayer Piezo Actuator,” PI motion and Position. [Online]. Available:

https://www.physikinstrumente.com/en/products/piezoceramic-actuators/linearactuators/p-088-round-picma-stack-multilayer-piezo-actuator-100821/

[117] R. Orszulik, J. J. Shan, Output Feedback Integral Control of Piezoelectric Actuator 
considering Hysteresis. Precision Engineering 2016, 47(1): 90-96.

[118] Y. F. Liu, J. J Shan, Y. Meng, and D. F. Zhu. Modeling and Identication of Asymmetric Hysteresis in Smart Actuators: a Modified Maxwell-slip Model Approach, IEEE/ASME Transactions on Mechatronics, 2016, 21 (1): 38-43.

[119] J. J. Shan, Y. F Liu, U. Gabbert, and N. G. Cui. Control System Design for Nanopositioning using Piezoelectric Actuators. Smart Materials and Structures, 25 (2): 025024, 2016.

[120] C. H. Lin, "LIGA-Like Techniques," Encycl. Microfluid. Nanofluidics, p. pp 10031005, 2008.

[121] M. Corporation, "SUMMiT V Five Level Surface Micromachining Technology Design Manual," Albuquerque, NM, 2012.

[122] "Fabricating MEMS and Nanotechnology," MNX, MEMS \& Nanotechnology Exchange. [Online]. Available: https://www.memsexchange.org/MEMS/fabrication.html.

[123] G. Dung, J. Su, H. Toshiyoshi, and M. C. Wu, "Surface-micromachined 2-D optical scanners with high-performance single-crystalline silicon micromirrors," IEEE Photonics Technol. Lett., vol. 13, no. 6, pp. 606-608, 2001.

[124] “MUMPs®.” [Online]. Available: http://www.memscap.com/products/mumps.

[125] H. J. Buchner and G. Jager, "A novel plane mirror interferometer without using corner cube reflectors," Meas. Sci. Technol., vol. 17, pp. 746-752, 2006.

[126] D. Kalamatianos, P. E. Wellsteadjohn, M. E. Liatsis, D. Kalamatianos, P. E. Wellstead, and J. M. Edmunds, "Active alignment for two-beam interferometers," Am. Inst. Phys., vol. 13103, pp. 1-13, 2006.

[127] C. G. Prevost, J. Genest, "Dynamic alignment of a Michelson interferometer using a position sensitive device," in Space Systems Engineering and Optical Alignment Mechanisms, 2004, vol. 5528, pp. 293-304.

[128] L. Genzel and J. Kuhl, "Tilt-compensated Michelson interferometer for Fourier transform spectroscopy," Opt. Soc. Am., vol. 17, pp. 3304-3308, 1978.

[129] M. Holler and J. Raabe, "Error motion compensating tracking interferometer for the position measurement of objects with rotational degree of freedom," Opt. Eng., vol. 54, pp. 1-7, 2015. 
[130] J. Ahn, J. Kim, C. Kang, J. Kim, and S. Kim, "High resolution interferometer with multiplepass optical configuration," Opt. Soc. Am., vol. 17, no. 23, pp. 2104221049, 2009.

[131] D. Fu, K. A. Walker, K. Sung, C. D. Boone, M. Soucy, and P. F. Bernath, "The portable atmospheric research interferometric spectrometer for the infrared, PARIS-IR,” J. Quant. Spectrosc. Radiat. Transf., vol. 103, pp. 362-370, 2007.

[132] B. Carli, A. Barbis, J. E. Harries, and L. Palchetti, "Design of an efficient broadband far-infrared Fourier-transform spectrometer," Opt. Soc. Am., vol. 38, no. 18, pp. 3945-3950, 1999.

[133] P. Hariharan, "Half-Shade Setting System for the Michelson Interferometer," in National Physical Laboratory, 1960, vol. 50, pp. 1026-1027.

[134] A. J. H. Meskers, D. Voigt, and J. W. Spronck, "Relative optical wavefront measurement in displacement measuring interferometer systems with sub-nm precision," Opt. Soc. Am., vol. 21, no. 15, pp. 17920-17930, 2013.

[135] D. E. Jennings, "Passive tilt compensation in an FTS using a double-sided flat retroreflector," Appl. Opt., vol. 27, no. 22, pp. 4605-4606, 1988.

[136] Q. Yang, B. Zhao, and D. Wen, "Principle and analysis of a moving double-sided mirror interferometer," Opt. Laser Technol., vol. 44, no. 5, pp. 1256-1260, 2012.

[137] R. Wei and B. Yin, "A tilt-compensated Fourier transform spectrometer with an image rotator,” Opt. Commun., vol. 346, no. July, pp. 115-119, 2015.

[138] "Off-Axis Parabolic Mirrors," Thorlabs, Inc. [Online]. Available: https://www.thorlabs.com/newgrouppage9.cfm?objectgroup_id=7002

[139] A. Cowen, B. Dudley, E. Hill, M. Walters, and R. Wood, "MetalMUMPs Design Handbook a MUMPs ${ }^{\circledR}$ process.” pp. 1-39, 2009.

[140] J. Judy, "Batch-fabricated ferromagnetic microactuators with silicon flexures," 1996.

[141] C. Liu and Y. W. Yi, "Micromachined magnetic actuators using electroplated Permalloy," IEEE Trans. Magn., vol. 35, no. 3, pp. 1976-1985, 1999.

[142] Serhan O. Isikman, O. Ergeneman, Arda D. Yalcinkaya, and H. Urey, "Modeling and characterization of soft magnetic film actuated 2-D scanners," IEEE J. Sel. Top. Quantum Electron., vol. 13, no. 2, pp. 283-289, 2007. 
[143] “CoventorWare," CoventorWare, 2012. [Online]. Available: http://www.coventor.com/coventorware.html.

[144] S. He, J. S. Chang, L. Li, and H. Ho, "Characterization of Young's modulus and residual stress gradient of MetalMUMPs electroplated nickel film," Sensors Actuators A Phys., vol. 154, no. 1, pp. 149-156, 2009.

[145] “Ansys." [Online]. Available: http://www.ansys.com/.

[146] “Zygo Nexview 3D optical surface profiler." [Online]. Available: http://www.zygo.com/?/met/profilers/nexview/.

[147] Y. Xue and S. He, "Microsystem Technologies Magnetic Field Assisted Bonding Technology for Released Micro Actuator and Mirror surface," J. Microsyst. Technol., 2016.

[148] "Solidworks." [Online]. Available: http://www.solidworks.com/.

[149] I. Zine-El-Abidine and M. Okoniewski, "A tunable radio frequency MEMS inductor using MetalMUMPs," J. Micromechanics Microengineering, vol. 17, no. 11, pp. 2280-2287, 2007.

[150] “Loctite 312 Technical Data Sheet," 2004. [Online]. Available: https://www.exdron.co.il/images/Products/files/Kit-Loctite-312-10ml-Primer-73640g-DG--datasheet.pdf.

[151] “Loctite 736 Technical Data Sheet," 2004. [Online]. Available: https://www.exdron.co.il/images/Products/files/Loctite-736-6oz-170ml-Spray73656--datasheet.pdf.

[152] “EPO-TEK 353ND." [Online]. Available: http://www.epotek.com/site/administrator/components/com_products/assets/files/S tyle_Uploads/353ND.pdf.

[153] Y. Xue, H. Zuo and S. He, "A repulsive magnetic force driven translation micromirror," J. Micromechanics Microengineering, in-press, 2017.

[154] R. Smith and F. K. Fuss, "Theoretical analysis of interferometer wave front tilt and fringe radiant flux on a rectangular photodetector.," Sensors (Basel)., vol. 13, pp. 11861-11898, 2013.

[155] D. N. Stacey, J. M. Huntley, T. Widjanarko, and P. D. Ruiz, "Straight, conic and circular fringes in single interferogram," Eur. J. Phys., no. 36, pp. 1-8, 2015. 
[156] “Michelson Interferometer- Note of Physics 3340," 2001.

[157] Mirrorcletech, "MEMS Mirrors." [Online]. Available: http://mirrorcletech.com/devices.html.

[158] A. R. Institute, “ARI MEMS Micromirror Demonstration Devices.” [Online]. Available: http://www.adriaticresearch.org/demos.htm.

[159] Innoluce, "1D MEMS mirrors." [Online]. Available: http://www.innoluce.com/\#products.

[160] T.s Stearns, Flexible Printed Circuitry. McGraw Hill Professional, 1996.

[161] "What are Flexible Printed Circuits." [Online]. Available: http://www.allflexinc.com/flexible-printed-circuits/.

[162] Thorslabs, "linear Stages.” [Online]. Available: https://www.thorlabs.com/newgrouppage9.cfm?objectgroup_id=5088.

[163] Thorlabs, "Manual Rotation Stages." [Online]. Available: https://www.thorlabs.com/newgrouppage9.cfm?objectgroup_id=992.

[164] T. D. Henning and J. L. Carlsten, "Cyclic shearing interferometer for collimating short coherence-length laser beams," vol. 31, no. 9, pp. 1199-1209, 1992.

[165] P. R. Griffiths, and J. A. de Haseth, "Theoretical background," in Fourier Transform Infrared Spectrometry, Second., A John Wiley \& Sons, INC., pp. 4650.

[166] P. L. King, M. S. Ramsey, P. F. McMillan, and G. Swayze, "Laboratory Fourier Transform Infrared Spectroscopy Methods for Geologic Samples," in Infrared Spectroscopy in Geochemistry, Exploration Geochemistry, and Remote Sensing, 2005. 